\title{
Mercury's Weather-Beaten Surface: Understanding Mercury in the Context of Lunar and Asteroidal Space Weathering Studies
}

\author{
Deborah L. Domingue • Clark R. Chapman • Rosemary M. Killen • \\ Thomas H. Zurbuchen • Jason A. Gilbert • Menelaos Sarantos • Mehdi Benna • \\ James A. Slavin • David Schriver • Pavel M. Trávníček • Thomas M. Orlando • \\ Ann L. Sprague · David T. Blewett · Jeffrey J. Gillis-Davis · William C. Feldman • \\ David J. Lawrence • George C. Ho • Denton S. Ebel • Larry R. Nittler • Faith Vilas • \\ Carle M. Pieters • Sean C. Solomon • Catherine L. Johnson • Reka M. Winslow • \\ Jörn Helbert • Patrick N. Peplowski • Shoshana Z. Weider • Nelly Mouawad • \\ Noam R. Izenberg • William E. McClintock
}

Received: 23 July 2013 / Accepted: 13 February 2014 / Published online: 24 April 2014

(C) The Author(s) 2014. This article is published with open access at Springerlink.com

\begin{abstract}
Mercury's regolith, derived from the crustal bedrock, has been altered by a set of space weathering processes. Before we can interpret crustal composition, it is necessary to understand the nature of these surface alterations. The processes that space weather the surface are the same as those that form Mercury's exosphere (micrometeoroid flux and solar
\end{abstract}

D.L. Domingue $(\varangle)$ · W.C. Feldman · F. Vilas · C.L. Johnson

Planetary Science Institute, 1700 E. Fort Lowell, Suite 106, Tucson, AZ 85719-2395, USA

e-mail: domingue@psi.edu

C.R. Chapman

Southwest Research Institute, 1050 Walnut Street, Suite 300, Boulder, CO 80302, USA

R.M. Killen · M. Benna

Solar System Exploration Division, NASA Goddard Space Flight Center, Greenbelt, MD 20771, USA

T.H. Zurbuchen · J.A. Gilbert · J.A. Slavin

Department of Atmospheric, Oceanic and Space Sciences, University of Michigan, Ann Arbor, MI 48109, USA

M. Sarantos

Heliophysics Science Division, NASA Goddard Space Flight Center, Greenbelt, MD 20771, USA

D. Schriver

Institute of Geophysics and Planetary Physics, University of California, Los Angeles, CA 90024, USA

P.M. Trávníček

Space Sciences Laboratory, University of California, Berkeley, CA 90704, USA

T.M. Orlando

School of Chemistry and Biochemistry and School of Physics, Georgia Institute of Technology, Atlanta, GA 30332-0400, USA 
wind interactions) and are moderated by the local space environment and the presence of a global magnetic field. To comprehend how space weathering acts on Mercury's regolith, an understanding is needed of how contributing processes act as an interactive system. As no direct information (e.g., from returned samples) is available about how the system of space weathering affects Mercury's regolith, we use as a basis for comparison the current understanding of these same processes on lunar and asteroidal regoliths as well as laboratory simulations. These comparisons suggest that Mercury's regolith is overturned more frequently (though the characteristic surface time for a grain is unknown even relative to the lunar case), more than an order of magnitude more melt and vapor per unit time and unit area is produced by impact processes than on the Moon (creating a higher glass content via grain coatings and agglutinates), the degree of surface irradiation is comparable to or greater than that on the Moon, and photon irradiation is up to an order of magnitude greater (creating amorphous grain rims, chemically reducing the upper layers of grains to produce nanometerscale particles of metallic iron, and depleting surface grains in volatile elements and alkali metals). The processes that chemically reduce the surface and produce nanometer-scale particles on Mercury are suggested to be more effective than similar processes on the Moon. Estimated abundances of nanometer-scale particles can account for Mercury's dark surface relative to that of the Moon without requiring macroscopic grains of opaque minerals. The

\section{A.L. Sprague}

Lunar and Planetary Laboratory, University of Arizona, Tucson, AZ 85721-0092, USA

D.T. Blewett · D.J. Lawrence · G.C. Ho · P.N. Peplowski · N.R. Izenberg

The Johns Hopkins University Applied Physics Laboratory, Laurel, MD 20723, USA

\section{J.J. Gillis-Davis}

Hawai'i Institute of Geophysics and Planetology, University of Hawai'i, Honolulu, HI 96822, USA

\section{D.S. Ebel}

Department of Earth and Planetary Sciences, Division of Physical Sciences, American Museum of Natural History, New York, NY 10023-5192, USA

L.R. Nittler · S.C. Solomon · S.Z. Weider

Department of Terrestrial Magnetism, Carnegie Institution of Washington, Washington, DC 20015, USA

C.M. Pieters

Department of Geological Sciences, Brown University, Providence, RI 02912, USA

S.C. Solomon

Lamont-Doherty Earth Observatory, Columbia University, Palisades, NY 10964, USA

C.L. Johnson · R.M. Winslow

Department of Earth, Ocean, and Atmospheric Sciences, University of British Columbia, Vancouver, BC, V6T 1Z4, Canada

J. Helbert

Institute for Planetary Research, DLR, Rutherfordstrasse 2, 12489 Berlin, Germany

N. Mouawad

Department of Natural Sciences, Lebanese American University, Beirut, Lebanon

W.E. McClintock

Laboratory for Atmospheric and Space Physics, University of Colorado, Boulder, CO 80303, USA 
presence of nanometer-scale particles may also account for Mercury's relatively featureless visible-near-infrared reflectance spectra. Characteristics of material returned from asteroid 25143 Itokawa demonstrate that this nanometer-scale material need not be pure iron, raising the possibility that the nanometer-scale material on Mercury may have a composition different from iron metal [such as $(\mathrm{Fe}, \mathrm{Mg}) \mathrm{S}]$. The expected depletion of volatiles and particularly alkali metals from solar-wind interaction processes are inconsistent with the detection of sodium, potassium, and sulfur within the regolith. One plausible explanation invokes a larger fine fraction (grain size $<45 \mu \mathrm{m}$ ) and more radiation-damaged grains than in the lunar surface material to create a regolith that is a more efficient reservoir for these volatiles. By this view the volatile elements detected are present not only within the grain structures, but also as adsorbates within the regolith and deposits on the surfaces of the regolith grains. The comparisons with findings from the Moon and asteroids provide a basis for predicting how compositional modifications induced by space weathering have affected Mercury's surface composition.

Keywords Mercury (planet) $\cdot$ Space weathering $\cdot$ Surface processes $\cdot$ Exosphere $\cdot$ Surface composition $\cdot$ Space environment

\section{Introduction}

Space weathering is a term used for the set of processes that alter over time the observable physical, chemical, and mineralogical properties of the surfaces of atmosphere-free bodies. Much of what we know about surface composition is inferred from remotely sensed observations, such as mineral composition from spectral reflectance measurements and elemental composition from X-ray, gamma-ray, and neutron emission measurements. Space weathering alters spectral signatures at visible to near-infrared (NIR) wavelengths by (1) darkening (lowering the spectral albedo), (2) reducing spectral contrast (diminishing the strength of absorption features), and (3) reddening of the spectrum (increasing the slope of the continuum reflectance with increasing wavelength) (McCord and Adams 1972a, 1972b; Fischer and Pieters 1994). Studies of lunar samples at ultraviolet (UV) wavelengths show that space weathering alters spectral signatures in this wavelength region by (1) brightening (increasing spectral albedo), (2) degrading the near- and mid-UV absorption edge, and (3) bluing (decreasing the slope of the continuum reflectance with increasing wavelength) (Hendrix and Vilas 2006; Hendrix et al. 2012). These effects must be factored into the interpretation of spectral measurements in terms of the specific mineral compositions of surface material. The removal of material (such as volatiles) will affect elemental composition determinations. In this paper we review space weathering on Mercury and its implications for the interpretation of surface reflectance and remote sensing of elemental composition.

Alteration by space weathering processes is tied to the exposure of a planetary surface to its space environment. For the Moon and Mercury, interplay between the space environment and the surface also generates and maintains a surface-bounded exosphere. There are complex links between the space environment, weathering processes, the exosphere, and the evolution of the regolith (the surficial layer of soil or particulates).

By "space environment" we include such exogenic agents as solar wind electrons and ions, solar photons, cosmic radiation, and impacting meteoroidal and cometary debris. The flux, energy, rate of impact, and composition of impacting particles all affect the type and magnitude of physical and chemical alteration of the surface, through such processes as sputtering, implantation, and volatilization. Also, a planet's magnetic field can shield the 


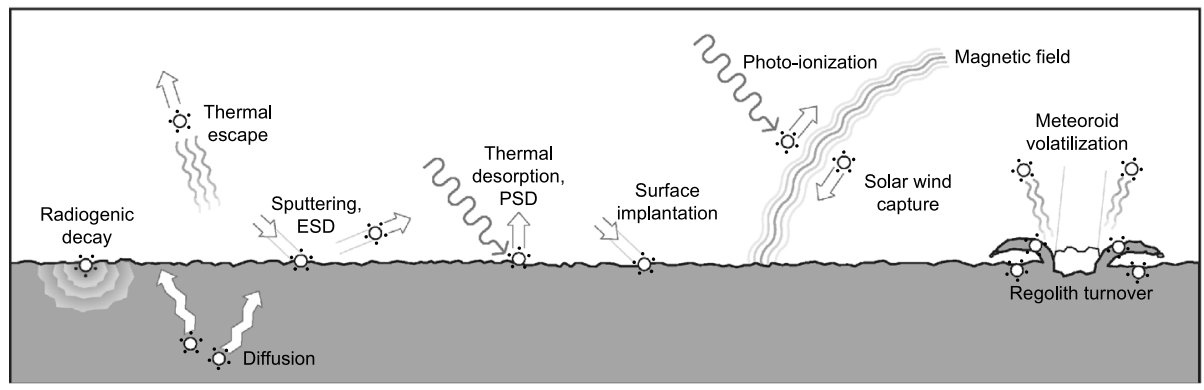

Fig. 1 Summary of the different processes that produce Mercury's exosphere and modify its surface. From Domingue et al. (2007)

surface or focus ions to specific regions, thus influencing the magnitude of space weathering effects and possibly introducing regional variations.

Processes within Mercury's system that link the exosphere, surface, and magnetosphere of Mercury are summarized in Fig. 1. Similar processes modify the lunar regolith and generate the lunar exosphere. This system is typically examined from a top-down or exospheric perspective, meaning that the effects of these processes on the physical, spatial, and temporal properties of the exosphere constituents are more often considered. Much of what has been learned about the interactions of these processes with the surface has been in an effort to understand the material that has been removed from the surface to form the exosphere. This paper examines the system from a reverse perspective in order to understand the material that is left on the surface and how it is altered by exosphere-generating processes. Whereas each process depicted in Fig. 1 affects the surface, the dominant alteration processes are thought to be micrometeoroid and ion bombardment (e.g., Hapke 2001). Because of Mercury's proximity to the Sun, such processes as photon-stimulated desorption (PSD), electron-stimulated desorption (ESD), and thermal desorption and cycling are also important. Of course, none of these processes works in isolation; their combined effects alter the physical, chemical, and spectral properties of the surface, yielding a set of changes termed maturation, and produce the observed exosphere.

Our understanding of the physical, chemical, and mineralogical effects of space weathering has come largely from the combined study of lunar samples and remote sensing observations of the Moon. Since the first Apollo sample return missions it has been noted that the spectrum of a typical lunar soil sample has a lower albedo, reddened spectrum, and subdued absorption bands compared with the spectrum of pulverized lunar rock of similar composition from the same landing site (Adams and Jones 1970; Hapke et al. 1970). Initial laboratory studies of lunar soils that included acid leaching (Gold et al. 1970; Hapke et al. 1970), Auger electron spectroscopy and X-ray photoelectron spectroscopy (Vinogradov et al. 1972; Gold et al. 1974, 1975, 1976; Housley and Grant 1975, 1976, 1977; Baron et al. 1977, 1978; Dikov et al. 1978), and electron spin resonance (Morris et al. 1975; Morris 1976, 1980) all indicated that the weathering agent responsible for the spectral effects was more abundant in the smaller size fractions of the soils and could be linked to the existence of reduced iron that is present within the upper nanometers of soil grains but not within grain interiors. Early transmission electron microscope (TEM) studies showed that a large fraction of lunar soil grains are covered with amorphous films a few hundred nanometers thick (Borg et al. 1971), but the TEM techniques then available were unable to resolve composition or structure within these films. Later re-examination of these films 
with more advanced TEM technology showed that the films differ in composition from the grains they coat and that they contain submicroscopic (on the scale of nanometers) grains of metallic iron (Keller and McKay 1993, 1997; Keller et al. 2000; Wentworth et al. 1999; Pieters et al. 2000). Today such nanometer-scale iron particles are considered the dominant contribution to the altered spectral properties of space-weathered soils.

Further insight into space weathering has come from comparisons between asteroid observations and meteorite measurements, and more recently, studies of samples returned from asteroid 25143 Itokawa (Noguchi et al. 2011, 2012, 2014) by the Haybusa spacecraft. The connection between ordinary chondrite (OC) meteorites and S-type asteroids has long been debated (e.g., Chapman 1996). The advent of spatially resolved spectral observations from spacecraft encounters with several S-type asteroids, such as 243 Ida by Galileo (Chapman 1996), 433 Eros by Near Earth Asteroid Rendezvous-Shoemaker (Clark et al. 2002), and Itokawa by Haybusa (Hiroi et al. 2006; Ishiguro et al. 2007; Noguchi et al. 2011, 2014) has led to the general acceptance that S-type asteroids are the parent bodies of OC meteorites and the spectral differences between parent bodies and the meteorites derived from them are ascribed to space weathering of the asteroid surfaces. The detection of space-weathered products in the Itokawa samples has further confirmed this relationship.

Asteroids span a considerable range of heliocentric distance (from rocky objects in the outer solar system and main-belt asteroids to Sun-grazing asteroids in the nearEarth population), so their surfaces have been collectively subjected to a wide range of fluxes and energies of micrometeoroids and solar-wind electrons and ions. These objects have long been known from spectral studies to experience space weathering processes (e.g., Chapman 2004). Much has been learned about variations in optical space weathering with asteroid size, location, composition, and age (Vernazza et al. 2009; Nesvorny et al. 2010). Asteroids vary in size from bodies that are sufficiently large to show spectral and elemental evidence (McCord et al. 1970; De Sanctis et al. 2012; Prettyman et al. 2012) that they have experienced magmatism and interior differentiation (e.g., Vesta) to small sub-kilometer objects. The larger asteroids have sufficient surface gravitational acceleration to sustain regolith processes similar to some of those on the Moon (Housen et al. 1979), whereas the smaller asteroids may lack well-developed regoliths of any type and are subject to processes peculiar to a microgravity environment (Scheeres et al. 2010). Asteroidal regoliths, like the lunar regolith, are also studied with laboratory samples (e.g., meteorites) but with the understanding that although gas-rich regolith-breccia meteorites retain some aspects of asteroid regoliths, they do not preserve the porous, particulate character of a surficial regolith. Most recently, studies of grains returned from the surface of asteroid Itokawa by the Hayabusa spacecraft showed that the samples are closely similar to LL ordinary chondrite materials, as predicted by Binzel et al. (2001), and contain nanophase Fe particles responsible for the space-weathered S-type spectrum of Itokawa (Noguchi et al. 2011). Examination of these asteroid grains also showed that the nanophase material includes particles of FeS and MgS as well as Fe (Noguchi et al. 2011, 2012, 2014).

Laboratory simulations of several space weathering processes conducted with meteorite samples and other materials have expanded our understanding of the effects of each process, the alteration rates, and their dependence on surface properties. Simulations of micrometeoroid impacts via pulsed laser experiments (Moroz et al. 1996; Yamada et al. 1999; Sasaki et al. 2001, 2003; Hiroi and Sasaki 2001; Brunetto et al. 2006a; Loeffler et al. 2008a, 2008b) have provided insight into the formation of vapor deposits and nanophase Fe production. Solar wind irradiation experiments have shown the role of sputtering in producing the space-weathered spectral changes in iron-bearing materials (Wehner 1964; Rosenberg and Wehner 1964; Hapke 1965, 1966, 1968, 1973, 2001; KenKnight et al. 1967; 
Nash 1967; Hapke et al. 1970, 1975; Yin et al. 1972, 1975; Yin and Tsang 1976; Dukes et al. 1999; Davoisne et al. 2008; Brunetto and Strazzulla 2005; Strazzulla et al. 2005; Brunetto et al. 2006b; Loeffler et al. 2009). Irradiation experiments on howardite, eucrite, and diogenite achondrite meteorites have confirmed a link between this class of meteorites and their postulated (V-type) asteroid parent bodies via space weathering of the asteroid surface (Fulivo et al. 2012). Experiments on additional exosphere production processes, such as electron-stimulated desorption (Yakshinskiy and Madey 2000, 2003, 2004, 2005; McLain et al. 2011) and thermal desorption (Shao and Paul 1993; Madey et al. 1998), have demonstrated the removal of material from samples and provide insight into the state of the regolith material remaining on the surface.

In this paper we examine Mercury's complex surface-exosphere-magnetosphere system in the context of what we have learned about space weathering from the lunar and asteroidal examples. Each process is discussed in terms of the relevant physics and the results of laboratory experiments. Mercury's particular space environment is considered, especially in light of observations during the 2008-2009 flybys by the MErcury Surface, Space ENvironment, GEochemistry, and Ranging (MESSENGER) spacecraft (Solomon et al. 2008) and during MESSENGER orbital operations at Mercury since March 2011. The role of each process in altering and maturing the surface is considered within the context of Mercury's environment. Mercury's surface composition is compared and contrasted with that of lunar surface materials and examined in light of inferred space weathering effects.

\section{Space Weathering Processes}

The processes in Fig. 1 fall into two main groups, those with micrometeoroid bombardment and those associated with radiation (charged particles and photons). Although we discuss these processes individually here, it is important to remember how each affects the other in understanding both exosphere formation and surface modification.

From laboratory measurements of lunar soil samples, most changes seen in spectra of space-weathered materials can be attributed to the presence of nanometer-scale particles of metallic iron (Papike et al. 1981; Pieters et al. 2000; Taylor et al. 2000). Such "nano-phase" iron $\left(\mathrm{npFe} \mathrm{p}^{0}\right)$ particles are a by-product of many of the processes in Fig. 1. Following the Apollo program, most asteroid regolith studies involved attempts to scale the developing understanding of lunar surface processes to bodies with lesser surface gravitational acceleration (Housen et al. 1979; Housen 1982; Langevin 1982), different exposure to the solar wind (e.g., Anders 1975), or a different impact environment. Most applications were to relatively large, main-belt asteroids. Concurrently, discrepancies between asteroidal and meteorite spectra led to speculations (Chapman and Salisbury 1973) that impact vitrificationthen the chief hypothesis for the reddening and darkening of lunar soils - might explain the differences between spectra of common ordinary chondrites and the somewhat redder and darker S-type asteroids, the most common type of asteroid in the inner main belt. Binzel et al. (1996) showed that spectra of moderate-albedo, near-Earth asteroids spanned a range between ordinary-chondrite-like spectra and S-type spectra, implying that asteroidal surfaces respond in an age-dependent way to a space-weathering process that causes their colors to evolve. Chapman (1996) showed that spectra of fresh ejecta from recent craters on asteroid Ida, as well as of Ida's satellite Dactyl, more closely resembled ordinary chondrite spectra than the more widespread, older S-type units on Ida, implying an ongoing space-weathering process.

Main-belt asteroid regoliths are less shocked than their lunar counterparts (due to lower impact velocities), and less impact melt (and related products such as agglutinates, 
Sect. 2.1.3) is produced (Hörz and Cintala 1997), as revealed by studies of gas-rich meteorite breccias, in which shock effects are mostly correlated with mechanical comminution (pulverization to smaller bits) and solid-state deformation (e.g., Stöffler et al. 1988, 1991; Keil et al. 1994). The rarity of agglutinitic particles in regolith breccia meteorites (Basu and McKay 1983; McKay et al. 1991; Noble et al. 2011) may also be due to the effects of differential melting and asteroid surface compositions. The scarcity of $\mathrm{npFe}^{0}$ particles in regolith breccia meteorites (Noble et al. 2011) indicates a different formation mechanism for $\mathrm{nFe}^{0}$ particles within asteroid regoliths than within the lunar regolith. Regardless, asteroid reflectance spectra exhibit characteristics of space weathering that can account for the spectral differences between ordinary chondrite meteorites and many S-type asteroids, their demonstrated parent bodies. A prime example of these effects is provided by the surface composition of Eros, an S-type asteroid. Eros has a composition similar to ordinary chondrites except for a marked depletion in sulfur (Trombka et al. 2000; Nittler et al. 2001; Foley et al. 2006) that can be attributed to space weathering processes.

Solar-wind ion irradiation has been argued to be the dominant mechanism for weathering asteroid surfaces (Pieters et al. 2000; Chapman 2004; Vernazza et al. 2009). Laboratory examination of grain samples returned from asteroid Itokawa show the presence of both $n p F e^{0}$ and nanophase iron and magnesium sulfides $[\mathrm{np}(\mathrm{Fe}, \mathrm{Mg}) \mathrm{S}]$ (Noguchi et al. 2011, 2014). The structure and composition of the rims (along with the texture of the nanophase particles) on ferromagnesian silicates within these samples suggests that $n p(\mathrm{Fe}, \mathrm{Mg}) \mathrm{S}$ near the surface is produced via vapor deposition associated with impact events, and that $\mathrm{nFFe}^{0}$ particles existing deeper within the rims are the result of radiation-induced amorphization and in situ reduction of Fe attributable to solar wind irradiation (Noguchi et al. 2011, 2014).

Laboratory studies (e.g., Clark et al. 2002) have shown that simulated space-weathering processes indeed modify mineral spectra in the manner observed for asteroids. They indicate that solar wind irradiation operates on time scales of $10^{4}-10^{6}$ y (Strazzulla et al. 2005; Vernazza et al. 2009), whereas micrometeoroid bombardment operates on longer timescales of $10^{8}-10^{9}$ y (Sasaki et al. 2001; Vernazza et al. 2009). Given dynamical analyses of the ages of family-forming collisional break-ups (e.g., Nesvorny et al. 2006), Vernazza et al. (2009) and others measured spectra of very young ( $<1$ My old) asteroids from collisional asteroid families and showed that most spectral changes take place over short time intervals (<1 My). Comparisons of the slope of the reflectance spectrum (between 500 and $900 \mathrm{~nm}$ wavelength) with family member age suggest that ion irradiation is rapid and dominates early in an asteroid's surface history, whereas slope changes due to micrometeoroid impacts are gradual and dominate later (Strazzulla et al. 2005; Vernazza et al. 2009). Willman et al. (2008) found that over long timescales ( $\sim 500 \mathrm{My})$ asteroid spectra gradually evolve from relatively mature (weathered) color properties to slightly more mature color properties as a steady state among processes is achieved.

More recent examination of spectral and color observations of fresh versus mature regolith on Vesta from the Dawn spacecraft indicates a weathering process that does not include $n \mathrm{pFe}^{0}$ but rather the presence of a finely dispersed, micrometer-size opaque phase (Pieters et al. 2012). Fresh craters on Vesta exhibit both bright and dark ejecta rays, neither of which is visible on older, morphologically degraded craters (Pieters et al. 2012). The maturation process on Vesta must therefore both brighten dark material and darken bright material. In addition, no spectral reddening is seen for older crater units compared with similar younger crater units (Pieters et al. 2012). These observations suggest that mechanical brecciation dominates over melting and vaporization during meteoroid and micrometeoroid impacts, making regolith mobility and fine-scale mixing an integral part of weathering on Vesta's surface (Pieters et al. 2012). It is the mobilization of the regolith, in addition to the 
comminution into a fine-grained particulate, which works to mature and homogenize Vesta's surface over time (Pieters et al. 2012).

In this section we examine each space weathering process in detail, and we discuss implications for exosphere production and surface modification. This section also includes a brief discussion of $\mathrm{nFFe}^{0}$ formation by each process and synthesizes results from lunar, asteroidal, and laboratory studies and their implications for Mercury. A more detailed discussion of the properties of $\mathrm{nFe}^{0}$ is given in a later section on Mercury's surface characteristics and composition.

\subsection{Micrometeoroid Bombardment}

Regolith formation is the product of the comminution of the local bedrock and boulders by repeated impacts. Whereas larger impacts dominate the excavation, fracturing, and comminution of bedrock, micrometeoroid $(<1 \mathrm{~cm}$ diameter) impacts process the surface on the scale of soil grains. There are four key effects associated with micrometeoroid bombardment that play a major role in the cycle of exosphere formation and surface maturation:

- Gardening

- Melt and vapor production

- Chemical reduction (change in oxidation state) and devolatilization

- Preferential processing by size and composition

The magnitude of each effect is governed by the micrometeoroid impactor flux (i.e., rate, given that the impactor size distribution is approximately constant between 0.1 and $1 \mathrm{AU}$ solar distance) and velocity, and by the target temperature and composition. These effects do not operate in isolation, so their mutual and combined effects must be considered. We consider each effect as it is understood on the basis of lunar studies. We then discuss differences, extrapolations, and predictions for Mercury's surface.

\subsubsection{Gardening}

Gardening is the continual process of burial and excavation by impacts of all sizes that affects (to various depths) altered or weathered material and brings unaltered (or less altered) material to the surface. Gardening tends to homogenize the regolith's top layers. In comparing and contrasting the micrometeoroid bombardment environments of the Moon and Mercury, Cintala (1992) calculated that the volume of material excavated by a dust particle (micrometeoroid to centimeter-size material) of a given size is $7 \%$ higher on Mercury than on the Moon. The higher impact velocity on Mercury is offset by the planet's higher surface gravitational acceleration, causing excavation (and retention) of material to be nearly equivalent, in contrast to melt and vapor production, which is much greater on Mercury (Cintala 1992; see following section). Thus, for a given flux, the gardening of the lunar and Mercury regoliths should be similar ( $<10 \%$ difference, Cintala 1992). A more recent study of impactors $>1 \mathrm{~cm}$ in diameter (Marchi et al. 2005a), which can strike Mercury at higher velocities, concluded that they can also lead to cause appreciable loss to interplanetary space of ejecta, only some of which later reaccretes (Gladman and Coffey 2009). Whatever the loss rate, the much greater impactor flux on Mercury compared with that on the Moon (discussed in the next section) results in a deeper regolith on Mercury due to the effects of impactors larger in size than $1 \mathrm{~cm}$.

For micrometeoroids $<1 \mathrm{~cm}$ in diameter, excavation (movement of material from depth) and retention of impactor material per impact is nearly equivalent at the Moon and Mercury 
(Cintala 1992). Lunar samples contain 1-4 \% of meteoritic material (Haskin and Warren 1991). The order of magnitude (or more) greater impact flux on Mercury (see next section) translates into an order of magnitude greater overturn rate and retention of impactor material within the regolith. Mercury's surface has thus been postulated to contain 5-20\% meteoroidal material (Noble et al. 2007) to a depth equivalent to that sampled on the Moon.

The efficiency of impact vaporization (and the other space weathering processes in Fig. 1) for generating the sodium exospheres on the Moon and Mercury is affected and limited by the diffusion of species from the interiors to the surfaces of regolith grains and by the regolith gardening rate. Killen et al. (2007) argued that the gardening rate on Mercury would be equivalent to the overturn of a 1-cm-thick layer of the regolith in $1.5 \times 10^{5} \mathrm{y}$ (compared with $10^{6} \mathrm{y}$ for the Moon, Heiken et al. 1991). Killen et al. (2004) argued further that regolith gardening on Mercury is sufficiently efficient to bring to the surface fresh grains that have not been completely depleted in $\mathrm{Na}$ (while burying depleted grains) in order to produce the currently observed exospheric $\mathrm{Na}$. In our surface-centric approach, this result implies that Mercury is being depleted in exospheric species (e.g., sodium, calcium, and magnesium) from both the immediate surface and to some depth in its regolith.

Thus, whereas a micrometeoroid impactor of a given impact mass may excavate nearly the same volume of regolith on Mercury and the Moon, the greater impact flux on Mercury will create a regolith that is potentially more mixed simply because of the increased rate of impacts. This mixing is one of the key components of the weathering process proposed for Vesta's surface (Pieters et al. 2012).

\subsubsection{Melt and Vapor Production}

The production of impact melt and vapor depends on the composition of impactor and target, the flux of impactors, and the impactor velocity distribution. We assume that impactor and surface compositions and impactor size distributions are, to first order, the same for the Moon and Mercury (later sections explore compositional differences).

From extrapolations of the observed meteor flux at Earth (Zook 1975), Cintala (1992) compared the flux and velocity distributions of dust impactors for the Moon and Mercury, with consideration of the varying spatial density of dust with distance from the Sun (Leinert et al. 1981). He found the flux at Mercury to be nearly 5.5 times greater than at the Moon and the mean impact velocity $60 \%$ greater. In contrast, from measurements of the mass accretion rate of cosmic dust at $1 \mathrm{AU}$ by the Long Duration Exposure Facility (LDEF) satellite (Love and Brownlee 1993), Borin et al. (2009) estimated that the mean impact velocity at Mercury is 30 \% higher than at the Moon and the mass flux at Mercury is 170 times that given by Cintala (1992) for the mean Mercury distance. The study by Marchi et al. (2005a) of impactors $>1 \mathrm{~cm}$ in diameter showed that these impactors strike Mercury at higher velocities ( $>20 \%$ strike at $>50 \mathrm{~km} / \mathrm{s}$ and at even higher speeds near Mercury's perihelion), enhancing melt and vapor production.

Mouawad et al. (2011) modeled both ground-based Mercury dayside observations acquired concurrently with nightside tail observations of exospheric sodium by MESSENGER's Mercury Atmospheric and Surface Composition Spectrometer (MASCS) (McClintock and Lankton 2007) and placed an upper limit on the contribution from impact vaporization of $2.1 \times 10^{6}$ atom $\mathrm{cm}^{-2} \mathrm{~s}^{-1}$. Burger et al. (2010) used Monte Carlo models to describe the distribution of neutral sodium measured by MASCS during the first two MESSENGER flybys of Mercury and derived an upper limit of a $15 \%$ contribution to the sodium exosphere by impact vaporization for the regions observed. Bounding the contribution of impact vaporization to exosphere generation also bounds the contribution of this process to 
the modification of the surface. If impact vaporization were the only source mechanism for sodium on the nightside/tail region (an upper limit model for impacts), then an impact flux on Mercury $\sim 6$ times the lunar value would account for the sodium measured by MASCS. Although this flux is within the range of impact vaporization rates of $\sim 0.6$ to $\sim 5$ times the lunar value as predicted by Morgan et al. (1988) and the flux and velocity predictions of Cintala (1992), it does not match the observed exosphere distribution on Mercury's dayside. An additional process, such as PSD, is required (Mouawad et al. 2011). In contrast, the much higher impact rate derived by Borin et al. (2009) ( 170 times that derived by Cintala 1992) does not reproduce either the amount or the distribution of sodium seen on either the dayside or nightside (Mouawad et al. 2011). Mouawad et al. (2011) estimated an upper limit on the contribution of impact vaporization to the generation of the exosphere, and thus its contribution to surface modification, a value more in line with those of Cintala (1992) than of Borin et al. (2009). Conversely, one possible component of impact vaporization, that of molecules, has not been considered and may at least partially reconcile the differences between the results of Cintala (1992) and Borin et al. (2009) (e.g., Berezhnoy and Klumov 2008).

Cintala (1992) showed that varying the impact velocity by a factor of 2 results in factors of $\sim 3$ variations in melt production and 4 to 5 in vapor production, so that $\sim 15$ and $\sim 20$ times more melt and vapor is produced, respectively, per unit area and unit time, at Mercury than at the Moon. The melt produces glass within the regolith, and the vapor produces coatings or patinas on nearby regolith particles. These coatings and patinas contain many of the volatiles released during the impact, melt, and vaporization process, creating a repository for these elements within the regolith. The vapor also contributes to the formation of additional glass, the redistribution of volatiles, and a net loss of volatiles from the surface.

\subsubsection{Glass Production and Properties}

Two types of glass in lunar soils are defined by differences in their shape and surface texture. The first, fairly regular spheres or ellipsoids with relatively smooth surfaces, are mostly free of clasts (rock or mineral fragments) and have chemically homogeneous interiors (e.g., Apollo Soil Survey 1971; Delano 1986; Hörz and Cintala 1997). These glass spherules can be either volcanic in origin or produced by micrometeoroid impacts onto crystalline rock surfaces (Hörz and Cintala 1997).

The second type of glass is irregular in shape, often jagged, clast-laden, and frequently vesicular (Hörz and Cintala 1997). This type of glass is found in agglutinates (McKay et al. 1972), which are glassy, fused local soils constituting a major component of lunar regolith material (McKay et al. 1991; Hörz and Cintala 1997). Agglutinates are theorized to form by the following impact process (McKay et al. 1991):

- A high-velocity micrometeoroid impacts soil containing previously implanted solar-wind particles, chiefly hydrogen and helium.

- Grains are thereby melted, forming glass, and implanted solar wind material is released.

- The released hydrogen reacts with FeO in the glass, reducing it to metallic iron and producing water, most of which escapes from the glass.

- Vesicles are formed in the glass by the released solar wind gases and possibly by released water vapor.

- Nanometer-sized iron droplets $\left(\mathrm{npFe}^{0}\right)$ formed by the reduction process are incorporated into the agglutinate.

- The glass melt engulfs local, small soil grains before it cools.

- The hot glassy melt releases solar-wind-produced gases from the engulfed soil grains, possibly trapping some of these gases in the glass as it cools. 

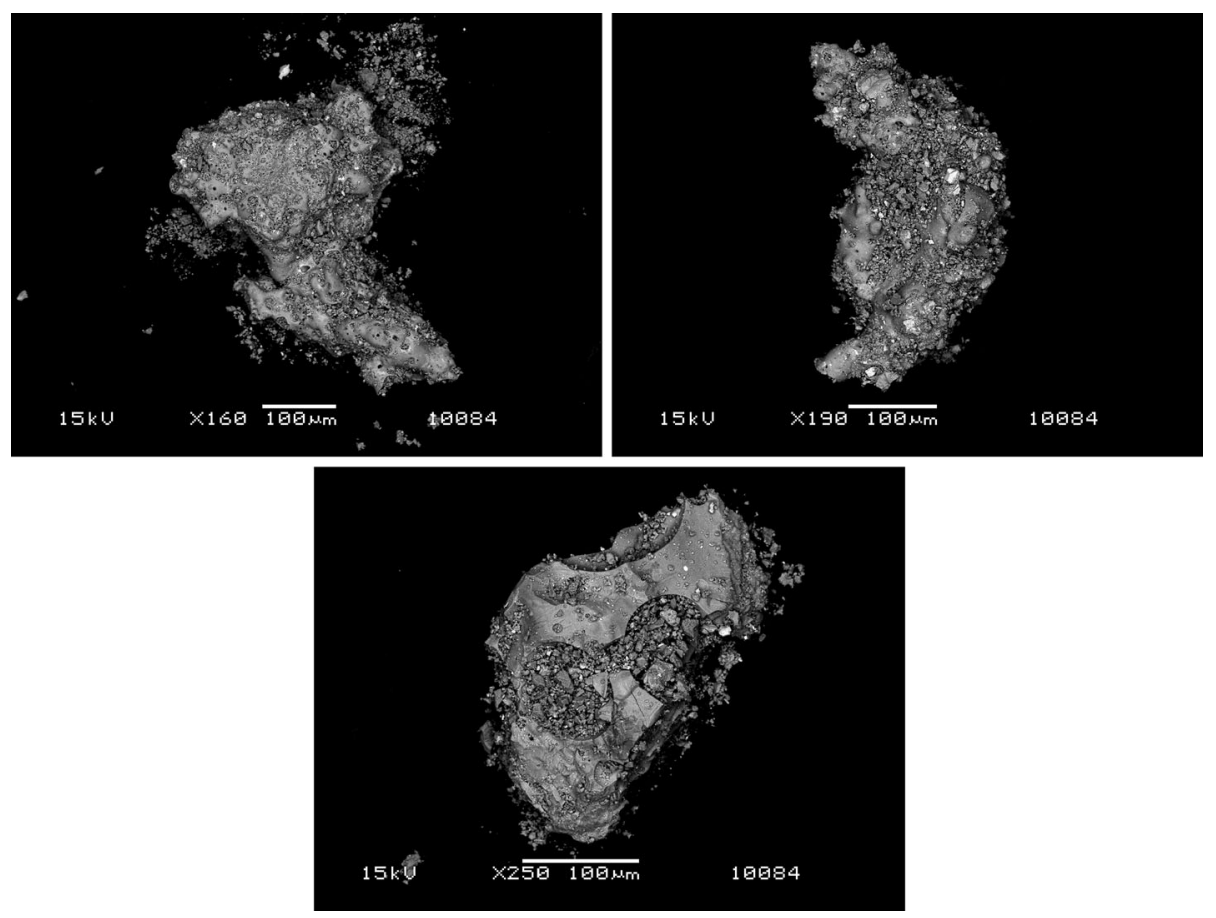

Fig. 2 Scanning electron microscope (SEM) images of typical lunar agglutinates extracted from Apollo 11 soil sample 10084. These photomicrographs show that the glassy surface is extensively coated with small, fine-grained soil fragments. These images demonstrate the irregular shapes, and often delicate structures, common to agglutinates. Visible on the surface of these agglutinates are regions of glassy, fragment-free surfaces adjacent to fragment-laden surfaces. Images courtesy of Sarah Noble (Goddard Space Flight Center)

This scenario includes the formation of water in addition to the production of metallic iron within these glasses. The presence of metallic iron has been verified (Keller and McKay 1993), but the production of water has not been verified by either laboratory experiments or measurements within lunar agglutinitic glass (Taylor et al. 1995). An alternate suggestion for the reduction of $\mathrm{FeO}$ is through interaction of solar wind carbon rather than hydrogen to produce the observed metallic Fe (Taylor et al. 1995).

Agglutinates constitute up to half of sieve fractions $<0.5 \mathrm{~mm}$ in size in many lunar soils (Heiken 1975; Morris et al. 1983; Hörz and Cintala 1997). Figure 2 shows examples of lunar agglutinates, revealing their shapes and textures. A ropy glass particle from an Apollo 12 soil sample, shown in Fig. 3, is considered to be an impact product (McKay et al. 1991). The scanning electron micrograph shows the complex surface texture resulting from the welded coating of fine fragments. Cintala (1992) demonstrated that the amount of melt produced on Mercury per gram of impactor is twice the lunar value. The majority of the impact energy goes into melting and vaporization. The higher melt production may enhance the concentration of glass within Mercury's regolith compared with lunar soils, so Cintala (1992) argued that little crystalline material is likely to survive in the upper layers of Mercury's regolith. Mercury's glassy regolith should be highly reworked by melting and vaporization, solar wind implantation, and the combination of these processes, thus reducing the $\mathrm{Fe}^{2+}$ content of the glasses (Cintala 1992). 
Fig. 3 Scanning electron photomicrographs of ropy glass grain from Apollo 12 soil sample 12033. (a) This micrograph (NASA photo S71-24593) shows the irregular twisted form of many of these grains. (b) This higher-resolution micrograph (NASA photo S71-24586) shows the texture of the grain surface, composed of a coating of tightly welded fine-fragment material. Images from McKay et al. (1991)
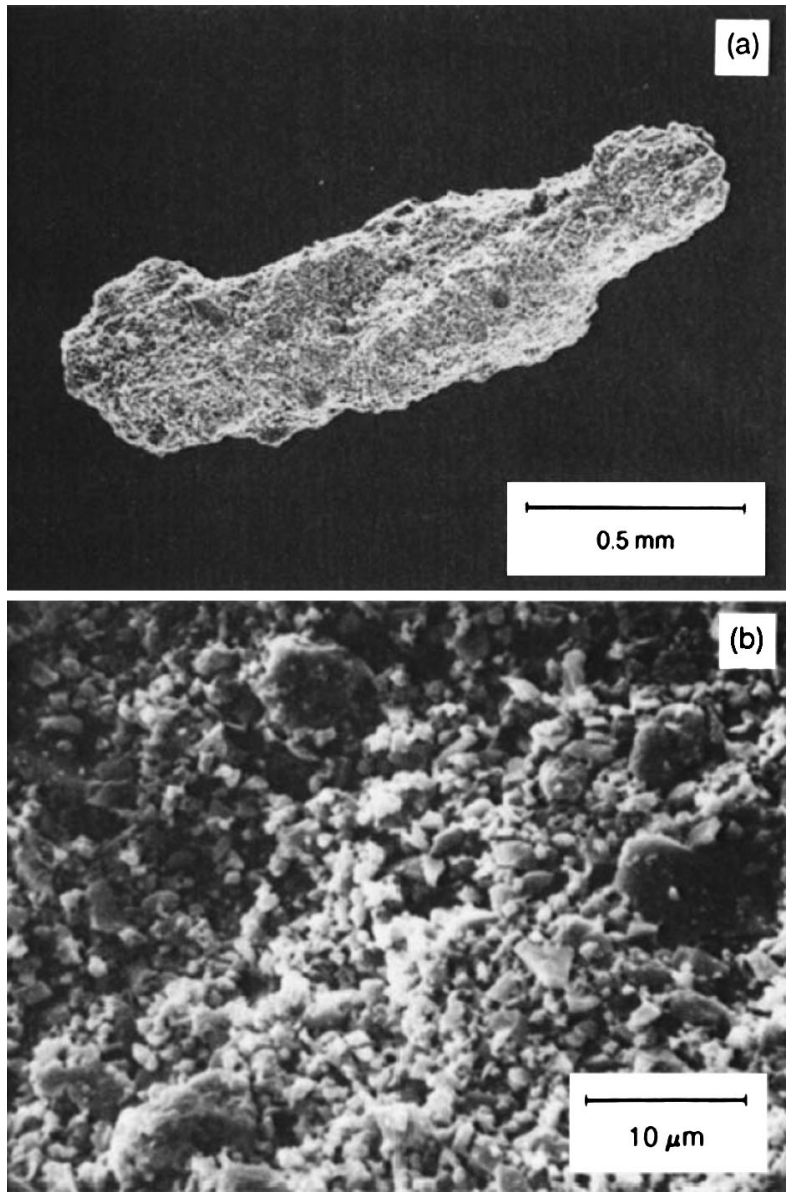

Impact-produced vapor also condenses on nearby regolith grains to produce amorphous (glass) coatings or patinas on the grains. In lunar soils these patinas are often chemically reduced compared with grain interiors and contain $n \mathrm{pFe}^{0}$. The size or depth of the vapor coating or rim is a function of the lifetime of the grain at the surface and the amount of vapor produced during the surface exposure time of the grain. Whereas Cintala (1992) argued that the rim depth will be very small in both the lunar and Mercury cases, his estimate of vapor production on Mercury is an order of magnitude greater, implying a larger source for coating adjacent grains, though the percentage of vapor produced that recondenses or is cold-trapped versus the fraction that escapes the system is unknown. The greater vapor abundance could potentially produce thicker rims on Mercury grains than on grains on the Moon, but it is difficult to factor in the number of impact events to which a grain is exposed during its surface-residence lifetime. Examples of glass rims on lunar soil grains are shown in Fig. 4.

\subsubsection{Material Reduction and Devolatilization}

Nano-phase metallic iron particles form on the Moon by reduction of $\mathrm{FeO}$ in lunar soils. Their presence causes most of the changes seen in space-weathered spectra (Papike et al. 1981; Pieters et al. 2000; Taylor et al. 2000). The particles occur in thin amorphous rims 
Fig. 4 (Top) SEM-based energy dispersive X-ray $\mathrm{Fe} \mathrm{K} \alpha$ image of a set of diverse grains from lunar soil 79221. These grains were extracted from the 10-20 $\mu \mathrm{m}$ size fraction of the soil. In this image, iron-bearing minerals such as olivine and orthopyroxene (Oliv and $O p x$, respectively) are bright. Plagioclase (Plag) contains very little iron as $\mathrm{FeO}$ and so appears dark. The enrichment of iron in the form of $n \mathrm{pFe}^{0}$ on the rims of the plagioclase grains is apparent in the bright rings around the grains (arrows). (Bottom) TEM image of an anorthitic plagioclase grain $(A n)$ from the same lunar soil sample. The small spheres of $n p F e^{0}$ within the rim of the grain (arrows) appear dark. Images from Pieters et al. (2000)
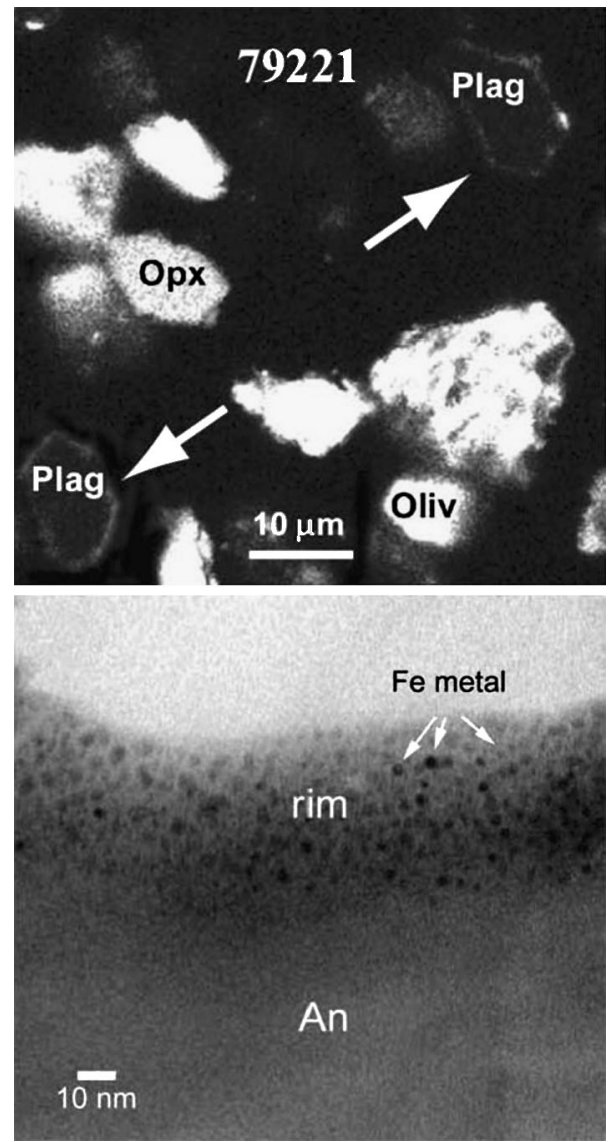

surrounding individual grains (Keller and McKay 1993) and as inclusions in the glass component of agglutinates. They exhibit two consistent properties: (1) they usually consist of relatively pure Fe, lacking the substantial $\mathrm{Ni}$ and Co present in meteoritic metal, and (2) most are extremely fine-grained. Ferromagnetic analysis shows that most metal is in the single-domain size range (3-10 nm) and thus is not always visible with an optical microscope (Morris 1976; Keller and McKay 1993). There is a bimodal distribution in $\mathrm{nFe}^{0}$ size depending on its location. Particles found in thin amorphous coatings on mineral grains are $\sim 3 \mathrm{~nm}$ in diameter (Keller and Clemett 2001), whereas those in agglutinates are approximately twice as large $(\sim 7 \mathrm{~nm})$ (Keller and Clemett 2001$)$. The TEM image of a lunar agglutinate in Fig. 5 shows the layer of fine-grained $\mathrm{nFFe}^{0}$ on the rim of the grain along with the coarser-grained $\mathrm{npFe}^{0}$ within the agglutinate's interior.

Nanometer-scale metallic iron is produced on the Moon mainly by (1) micrometeoroid bombardment and (2) solar wind ion implantation and sputtering. The two processes need not operate independently. We discuss production first in agglutinates and then in grain rims. Three models involving micrometeoroid bombardment have been advanced to explain the properties of $\mathrm{nFe}^{0}$ in agglutinates, each requiring melting of the soil grains to form glass. The first model invokes the liberation of previously implanted solar wind $\mathrm{H}$ and $\mathrm{He}$ during target melting. The liberated $\mathrm{H}$ reacts with $\mathrm{FeO}$ in the glass, partly reducing it to metallic 
Fig. 5 This TEM image of a lunar agglutinate grain shows the variation in $\mathrm{npFe}^{0}$ size within agglutinates. The arrows indicate the multiple layers of fine-grained $n p F e^{0}$ along the grain rim. The larger, dark spheres in the interior of the grain are coarse-grained $\mathrm{npFe}^{0}$ particles that are several times larger than the $\mathrm{nFe}^{0}$ seen within the grain rim. Image from Pieters et al. (2000)

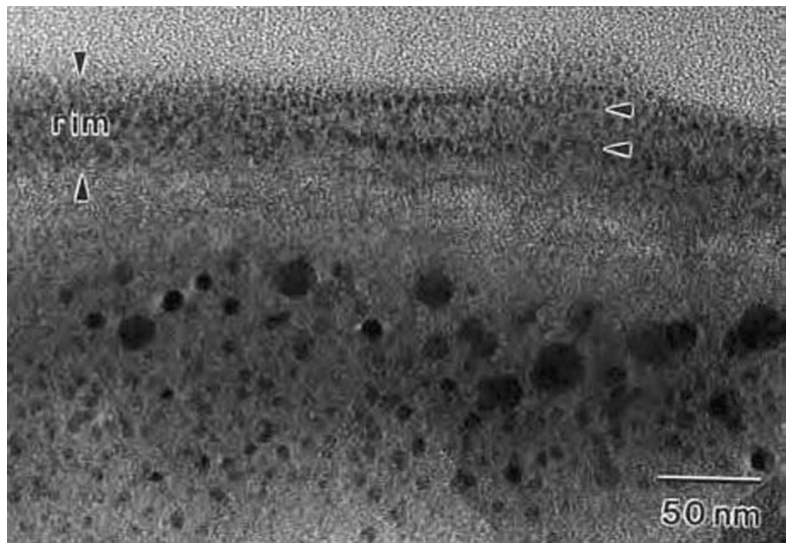

iron particles and producing some $\mathrm{H}_{2} \mathrm{O}$, which escapes from the glass (Housley et al. 1973a, 1973b).

In the second model, the intense, transient shock wave of an impact vaporizes volatile elements in the regolith. Repeated impacts progressively deplete the maturing soil in more volatile elements, including oxygen, so that a mature regolith is already reduced; subsequent melting that forms agglutinates will simultaneously create $\mathrm{npFe}^{0}$ (Hapke 2001). Laboratory measurements show (Epstein and Taylor 1972) that vaporization of the light isotopes ${ }^{16} \mathrm{O}$ and ${ }^{28} \mathrm{Si}$ (or, conversely, a preferential condensation of the heavy isotopes, ${ }^{18} \mathrm{O}$ and ${ }^{30} \mathrm{Si}$ ) is accompanied by a reduction of the bulk O/Si ratio (Hapke 2001).

The third model is a two-stage process. First, micrometeoroid bombardment melts the finest fraction of the regolith (Papike et al. 1981; Pieters et al. 2000; Taylor et al. 2000), which has the highest concentration of $\mathrm{nFFe}^{0}$ within particle rims. Agglutinates are thus enriched in $\mathrm{npFe}^{0}$ by the melting and incorporation of grains that already include $n p F e^{0}$; concentration then "coarsens" or enlarges the $\mathrm{npFe}^{0}$ grains within agglutinates (Pieters et al. 2000; Noble and Pieters 2003).

Although there is debate about which mechanism dominates in reducing $\mathrm{Fe}^{2+}$ to $\mathrm{npFe}^{0}$, in all three models the production of nanophase Fe within agglutinates depends on both the availability of $\mathrm{Fe}$ compounds in target soils and melting by micrometeoroid impacts.

Turning to formation of $\mathrm{nFe}^{0}$ within grain rims, there are also three models. The first two include impact-produced vapor deposition. In these models, vapor condenses on nearby particles and creates a patina or rim on regolith grains, but a reducing environment as a result of previously implanted solar $\mathrm{H}$ leads to formation of $\mathrm{npFe}^{0}$. Alternatively, the vapor is produced at a sufficiently high temperature that hydrogen implantation is not required to reduce the FeO present to metallic iron (Sasaki et al. 2001; Sasaki and Kurahashi 2004). Formation of vapor-deposit rims is a surface effect. Since the ratio of surface to volume is greatest within the finer soil fractions, it is understandable that increased $\mathrm{npFe}^{0}$ concentrations are found within the finer fractions of lunar soil samples.

The final model for producing $\mathrm{nFe}^{0}$ within grain rims (invoked to explain space weathering on asteroids) does not require micrometeoroid impact or vapor production but rather involves the impact of solar wind ions on the top layers of regolith grains. According to this idea, the bombardment of regolith grains by solar wind hydrogen produces a reducing environment. If $\mathrm{FeO}$ is present, then hydrogen implantation will reduce the $\mathrm{FeO}$ to $\mathrm{Fe}^{0}$, forming single-domain, nanometer-scale metallic iron particles within the upper layers of the regolith grains (Hapke 2001). Examination of the Itokawa samples indicates that within the particle 
rims that contain $n \mathrm{pFe}^{0}$ there are nearby zones that show relative depletion in Fe, indicating that the $\mathrm{nFFe}^{0}$ is formed in situ (Noguchi et al. 2014).

\subsubsection{Preferential Processing by Size and Composition}

Not all minerals behave in the same manner in response to comminution, melting, and vaporization processes. There is evidence in the lunar soils, for instance, of mechanical fractionation by impacts. Lunar soils show feldspar enrichment in the smaller size fractions relative to other minerals (Devine et al. 1982; Papike et al. 1982; McKay et al. 1991), attributed to the excellent cleavage inherent in plagioclase feldspars compared with other minerals (Basu et al. 1975; Basu 1976; Hörz et al. 1984). Minerals that occur as very small grains in the parent rock are also concentrated in the finer fractions after comminution (e.g., Laul et al. 1981). Such effects are important because the fine fractions $(<45 \mu \mathrm{m})$ dominate the spectral reflectance properties of lunar soils (Pieters 1983, 1993; Pieters et al. 1993; Hapke 2001).

Differential melting (compositionally dependent contributions to melts) also occurs (Taylor et al. 2001a, 2001b, 2010) and is important in forming glasses, especially agglutinates. Models of lunar soil evolution incorporate differential melting to explain the following soil observations (Taylor et al. 2001a, 2001b, 2010):

- The agglutinitic glass component in soil separates increases with decreasing size fraction.

- The agglutinitic glass compositions form an apparent mixing line between bulk highland and mare soil compositions.

- Agglutinitic glasses in mare soils are depleted in $\mathrm{TiO}_{2}$.

- Agglutinitic glasses in highland soils are rich in basaltic components ( $\left.\mathrm{FeO}, \mathrm{TiO}_{2}, \mathrm{MgO}\right)$.

Pieters and Taylor (2003) suggested that these observations can be explained by a combination of mare-highland mixing and a proposed differential melting sequence. For a given temperature and time the relative contribution to the melt composition from the different compositional components of the regolith are provided in the order glass $>$ plagioclase $>$ pyroxene $>$ ilmenite, where glass is the first or highest contributor and ilmenite is the last or least contributor.

Lunar soils also exhibit the effects of vapor fractionation. Impact-induced shock-wave heating vaporizes the more volatile elements (such as $\mathrm{H}, \mathrm{O}, \mathrm{S}$, and alkali metals) in the regolith. Some of the vaporized atoms escape lunar gravity, so the soil becomes progressively more depleted in volatiles with increasing maturity (McKay et al. 1991). Non-escaping volatiles undergo a measurable mass fractionation of their isotopes. Epstein and Taylor (1972) showed that lunar soils are extremely enriched in the heavier oxygen $\left({ }^{18} \mathrm{O}\right)$ and silicon $\left({ }^{30} \mathrm{Si}\right)$ isotopes relative to crystalline rocks, and these enrichments are directly related to the amount of hydrogen in the soils (McKay et al. 1991). They also showed that enrichment of ${ }^{18} \mathrm{O}$ and ${ }^{30} \mathrm{Si}$ is a surface effect and that oxygen preferentially escapes relative to silicon in the fractional vaporization process (McKay et al. 1991), contributing to a chemically reduced environment and production of $\mathrm{npFe}^{0}$. This fractionation process will also occur on Mercury, but the higher surface gravitational acceleration will inhibit the loss of lighter relative to heavier isotopes and of oxygen relative to silicon. Within the lunar samples there is also an increased concentration of volatile elements (such as $\mathrm{Zn}, \mathrm{Ga}, \mathrm{Ge}, \mathrm{Cd}, \mathrm{Sb}, \mathrm{Te}$, and $\mathrm{Hg}$ ) with decreasing soil size fraction (McKay et al. 1991). Such elements deposited on grain surfaces are thus enriched in the finer fractions due to their larger surface-to-volume ratios (Krähenbühl et al. 1977; McKay et al. 1991). This enrichment of volatiles within the finer soil fractions should also be operative on Mercury. 


\subsection{Solar Irradiation}

In addition to micrometeoroids, solar wind ions bombard and mature the surface of the Moon and are a major agent for altering asteroidal surfaces. The lunar surface is also subjected to bombardment by magnetospheric ions during its traverses of Earth's magnetosphere. Mercury's surface is bombarded by both solar wind particles and ions from its own magnetosphere. In order to better gauge the relative roles and alteration rates of ion and micrometeoroid bombardment on Mercury, we must understand the physical, chemical, and mineralogical changes induced by each process. Although many observational and laboratory studies of particle irradiation pertain to the generation of exospheres, we now examine corresponding surface effects in our surface-centric approach resulting from the processes of ion implantation, physical sputtering, chemical sputtering, desorption induced by electronic transitions (DIET), and thermal desorption.

\subsubsection{Ion Implantation}

Bombardment of solar wind or magnetospheric ions onto the planet's surface results in some combination of implantation and sputtering. This section examines surface alteration and maturation by ion implantation, and following sections examine sputtering by both physical and chemical mechanisms. Ion implantation refers to a collision between an impacting ion and a target regolith grain that results in the accommodation of the impacting ion within the grain's atomic lattice. Behrisch and Wittmaack (1991) demonstrated that solar wind protons at $\mathrm{keV}$ energies implant themselves to depths of a few tens of nanometers into lunar soil regolith grains, so implantation is mainly a surface effect. As such ions travel through grains they produce lattice defects along their trajectories. High-energy ions ( $>50 \mathrm{keV} /$ nucleon), such as cosmic rays, can penetrate deeper into the grain lattice structure. These lattice defects provide additional conduits that enhance diffusion of volatiles from grain interiors to their surfaces, which in turn enhances desorption processes and also provide sites for adsorption of atoms, especially from the exosphere.

Hydrogen is present in lunar soil samples that have been exposed to the solar wind (DesMarais et al. 1974; Epstein and Taylor 1975; Crider and Vondrak 2003) and is found mostly in the top $50 \mathrm{~nm}$ of mature lunar grains (Crider and Vondrak 2003). Solar wind proton bombardment begins with the proton implanting into the surface of a grain, where it picks up an electron, producing a hydrogen atom and reducing an atom in the outer layers of the grain. The chemical reduction (discussed in detail below) produces $\mathrm{OH}$, $\mathrm{H}_{2} \mathrm{O}$, and $n \mathrm{pFe}^{0}$. It also produces damaged or open atomic bonds, thus creating sites for adsorption of gaseous exospheric molecules (such as $\mathrm{H}_{2} \mathrm{O}$ ). Hydrogen content is correlated with soil exposure age and maturity in lunar samples (DesMarais et al. 1974; Crider and Vondrak 2003) and with temperature (Feldman et al. 2000); it plateaus around 50-75 ppm within very mature, equatorial soils (Morris 1976; Crider and Vondrak 2003) and, on average, is about $100 \mathrm{ppm}$ higher within the higher-latitude, colder soils (Feldman et al. 2000), which implies that a steady state is achieved between $\mathrm{H}_{2}$ release and $\mathrm{H}_{2}$ generation in mature regolith (Crider and Vondrak 2003).

Although ion implantation generates $\mathrm{H}_{2}$ in soils (Crider and Vondrak 2003), $\mathrm{H}_{2}$ is released via several mechanisms, including diffusion, sputtering, and vaporization. The diffusion rate for hydrogen in lunar soils is thought to be small, so the release of hydrogen is dominated by sputtering and vaporization. Vaporization of hydrogen occurs predominantly during the micrometeoroid impact process, discussed above. A steady-state $\mathrm{H}_{2}$ soil content, therefore, is achieved through a balance of generation and release rates. 
This steady state is consistent with the detection of hydroxyl and water signatures over the lunar surface (Vilas et al. 2008; Clark 2009; Pieters et al. 2009; Sunshine et al. 2009; McCord et al. 2011). Extrapolations to Mercury must account for several factors. Dayside temperatures on Mercury are much higher than on the Moon, making thermal desorption of implanted ions more efficient, perhaps to the point of depleting the surface of volatiles on short timescales. Regolith porosities and the fraction of fine-grained material influence the relative efficiencies of removal and trapping of ions. Impact studies (e.g., Cintala 1992) indicate a higher fraction of fine soil grains on Mercury, so calculations of the relative retention rates should account for these differences.

\subsubsection{Physical Sputtering}

The collision of energetic solar wind or magnetospheric ions with surface grains can release atoms via momentum transfer. Such physical sputtering is a surface effect, acting on the topmost atomic layers. Physical sputtering can release any atomic species, so elements contributed to the exosphere by sputtering reflect surface composition on an atomic level (Johnson et al. 2002; Killen et al. 2007; Wurz et al. 2010), though not in relative abundances. Several studies (Killen et al. 2007; Wurz et al. 2007, 2010) compared exospheric compositions expected from physical sputtering, photon-stimulated desorption, and impact vaporization with measured density and spatial distributions within the exosphere. All of these processes will produce an exosphere having a composition generally related to Mercury's surface composition, but the relative contributions of each process to exospheric production are highly inter-dependent. As for the material remaining on the surface, preferential sputtering of elements or molecules with high sputtering yields $(\mathrm{H}, \mathrm{O}$, and $\mathrm{OH}$, for example) will leave the topmost atomic layers enhanced in elements with lower sputtering yields ( $\mathrm{Fe}$ and Ti, for example) (Killen et al. 2007). Calculations of this effect to date have been based on the assumption that Mercury's regolith is composed predominantly of silicates, as the sputtering yields will vary with the mineral matrix. It has been proposed that physical sputtering reduces and disorders the topmost mono-layers, thus producing amorphous rims (e.g., Hapke 2001). Reduction of $\mathrm{Fe}^{2+}$ in iron-bearing grains by physical sputtering is therefore another route for producing $\mathrm{npFe}{ }^{0}$.

The rate at which grains within a surface regolith are modified depends on several variables. These grain modifications include sputtering and vaporization of material from the grain in addition to amorphization and coating of the grain rims by radiation, micrometeoroid impact, and melt deposition. The variables relevant to physical sputtering include the grain or surface composition, the gardening or regolith overturn rates, the rates of diffusion of elements from grain interiors to grain surfaces as well as volume diffusion rates within the regolith, the composition and energy of impacting ion species (properties of the magnetopshere or solar wind), and the flux of ions to the surface (configuration of the magnetosphere).

From an adopted mineralogical surface composition for Mercury (Table 1) derived from telescopic and MESSENGER measurements acquired during the Mercury flybys (summarized in Table 1) and average solar wind characteristics, Wurz et al. (2010) calculated a global physical sputtering rate comparable with the lunar rate. The escape rate for Mercury, however, is lower than for the Moon; because of Mercury's stronger surface gravitational acceleration, not all particles released by sputtering and micrometeoroid impact escape to the exosphere (Wurz et al. 2010). Starting with the mineralogical compositions in Table 1, Wurz et al. (2010) derived possible surface elemental compositions (Table 2) and calculated exospheric species yields from micrometeoroid impact vaporization, physical sputtering, 
Table 1 Estimated mineralogical composition for exospheric yields ${ }^{\mathrm{a}}$

\begin{tabular}{|c|c|c|}
\hline Mineral & $\begin{array}{l}\text { Chemical } \\
\text { composition }\end{array}$ & $\begin{array}{l}\text { Mineral abundance } \\
\text { (abundance range) } \\
\text { (mol\%) }\end{array}$ \\
\hline Iron/nickel metal & $\mathrm{Fe}, \mathrm{Ni}$ & $0.07(0.04-0.15)$ \\
\hline Trolite & $\mathrm{FeS}$ & $0.15(0-0.5)$ \\
\hline Daubreelite & $\mathrm{FeCr}_{2} \mathrm{~S}_{4}$ & $0.15(0-0.3)$ \\
\hline Oldhamite & $\mathrm{CaS}$ & $0.15(0-0.3)$ \\
\hline Sphalerite & $\mathrm{ZnS}$ & $0.58(0-1)$ \\
\hline Ilmenite & $\mathrm{FeTiO}_{3}$ & $0.07(0.02-0.15)$ \\
\hline Apatite & $\mathrm{Ca}_{5}\left(\mathrm{PO}_{4}\right)_{3} \mathrm{OH}$ & $1.45(0-2)$ \\
\hline \multicolumn{3}{|l|}{ Feldspar Group } \\
\hline Albite & $\mathrm{NaAlSi}_{3} \mathrm{O}_{8}$ & $17.44(13-21)$ \\
\hline Orthoclase & $\mathrm{KAlSi}_{3} \mathrm{O}_{8}$ & $0.39(0.2-0.7)$ \\
\hline Anorthite & $\mathrm{CaAl}_{2} \mathrm{Si}_{2} \mathrm{O}_{8}$ & $8.72(6.7-11)$ \\
\hline \multicolumn{3}{|l|}{ Pyroxene Group } \\
\hline Wollastonite & $\mathrm{CaSiO}_{3}$ & $2.91(2.4-3.5)$ \\
\hline Ferrosilite & $\mathrm{Fe}_{2} \mathrm{Si}_{2} \mathrm{O}_{6}$ & $0.36(0.1-0.5)$ \\
\hline Enstatite & $\mathrm{Mg}_{2} \mathrm{Si}_{2} \mathrm{O}_{6}$ & $29.06(24-34)$ \\
\hline \multicolumn{3}{|l|}{ Olivine Group } \\
\hline Fayalite & $\mathrm{Fe}_{2} \mathrm{SiO}_{4}$ & $2.18(1.7-2.7)$ \\
\hline Forsterite & $\mathrm{Mg}_{2} \mathrm{SiO}_{4}$ & $36.33(31-41)$ \\
\hline
\end{tabular}

${ }^{\mathrm{a}}$ From Table 1 of Wurz et al. (2010)

and PSD (discussed in Sect. 2.2.4) in order to understand the relative contribution of each process to exosphere formation. The elemental abundances adopted by Wurz et al. (2010) were not too different from those measured by MESSENGER's elemental remote sensing instruments (Sect. 4.3), though the sulfur component was underestimated. The results from Wurz et al. (2010), summarized in Table 3, provide estimates of the relative contributions to the exosphere from each of the above processes.

The results of Wurz et al. (2010) indicate that micrometeoroid impact vaporization and physical sputtering contribute comparably (to within an order of magnitude) to the removal of $\mathrm{O}, \mathrm{OH}, \mathrm{Mg}, \mathrm{K}, \mathrm{Ca}, \mathrm{Ti}, \mathrm{Cr}$, and $\mathrm{Zn}$. Micrometeoroid impact vaporization may remove an order of magnitude more $\mathrm{Na}, \mathrm{Al}, \mathrm{Si}, \mathrm{P}, \mathrm{S}$, and Fe than physical sputtering, but the model of Wurz et al. (2010) does not account for the influence of Mercury's internal magnetic field (discussed in Sect. 3.4.1). The two elements for which estimates of removal by PSD were made $(\mathrm{Na}$ and $\mathrm{K}$ ) show that this process dominates by about three orders of magnitude over either micrometeoroid impact vaporization or physical sputtering. Results from other simulation studies, however, suggest that impact vaporization is a significant contributor. Contributions from impact vaporization range from about $20 \%$ as much $\mathrm{Na}$ as PSD (Burger et al. 2010; Mouawad et al. 2011) to exospheric Na being totally produced via impact vaporization (Morgan et al. 1988; Borin et al. 2010). Regardless, these simulations predict that impact vaporization produces significantly more exospheric Na than that suggested by Wurz et al. (2010). 
Table 2 Estimated surface elemental composition (atom\%) for the calculation of exospheric yields ${ }^{\mathrm{a}}$

\begin{tabular}{lcc}
\hline Element & Additive model & Multiplicative model \\
\hline $\mathrm{O}$ & 58.61 & 58.80 \\
$\mathrm{Na}$ & 1.34 & 1.34 \\
$\mathrm{Mg}$ & 16.2 & 16.1 \\
$\mathrm{Al}$ & 2.71 & 2.64 \\
$\mathrm{Si}$ & 17.4 & 17.4 \\
$\mathrm{P}$ & 0.208 & 0.208 \\
$\mathrm{~S}$ & 0.519 & 0.529 \\
$\mathrm{~K}$ & 0.030 & 0.030 \\
$\mathrm{Ca}$ & 1.670 & 1.269 \\
$\mathrm{Ti}$ & 0.015 & 0.015 \\
$\mathrm{Cr}$ & 0.042 & 0.042 \\
$\mathrm{Fe}$ & 0.872 & 1.279 \\
$\mathrm{Ni}$ & 0.004 & 0.004 \\
$\mathrm{Zn}$ & 0.291 & 0.291 \\
$\mathrm{OH}$ & 0.069 & 0.069 \\
\hline
\end{tabular}

${ }^{\mathrm{a}}$ From Table 2 of Wurz et al. (2010)

Notes: The additive model is based on absolute values of the mineral elemental abundances. The multiplicative model is based on the ratio of the mineral elemental abundances

Table 3 Calculated surface density $\left(\right.$ atom $\mathrm{m}^{-3}$ ) of exospheric species for each generation process ${ }^{\mathrm{a}, \mathrm{b}}$

\begin{tabular}{llll}
\hline Element & $\begin{array}{l}\text { Micrometeoriod impact } \\
\text { vaporization }\end{array}$ & Physical sputtering & $\begin{array}{l}\text { Photon-stimulated } \\
\text { desorption }\end{array}$ \\
\hline $\mathrm{O}$ & $8.21 \times 10^{7}, 8.23 \times 10^{7}$ & $2.20 \times 10^{7}, 2.2 \times 10^{7}$ & N/A \\
$\mathrm{OH}$ & $9.96 \times 10^{4}, 9.96 \times 10^{4}$ & $2.42 \times 10^{4}, 2.42 \times 10^{4}$ & \\
$\mathrm{Na}$ & $2.25 \times 10^{6}, 2.25 \times 10^{6}$ & $9.66 \times 10^{5}, 9.66 \times 10^{5}$ & $1.97 \times 10^{9}, 1.97 \times 10^{9}$ \\
$\mathrm{Mg}$ & $2.79 \times 10^{7}, 2.77 \times 10^{7}$ & $1.09 \times 10^{7}, 1.08 \times 10^{7}$ & \\
$\mathrm{Al}$ & $4.93 \times 10^{6}, 4.81 \times 10^{6}$ & $7.23 \times 10^{5}, 7.04 \times 10^{5}$ & \\
$\mathrm{Si}$ & $3.23 \times 10^{7}, 3.23 \times 10^{7}$ & $3.30 \times 10^{6}, 3.30 \times 10^{6}$ & \\
$\mathrm{P}$ & $4.05 \times 10^{5}, 4.05 \times 10^{5}$ & $5.94 \times 10^{4}, 5.94 \times 10^{4}$ & \\
$\mathrm{~S}$ & $1.03 \times 10^{6}, 1.05 \times 10^{6}$ & $2.32 \times 10^{5}, 2.36 \times 10^{5}$ & \\
$\mathrm{~K}$ & $6.57 \times 10^{4}, 6.57 \times 10^{4}$ & $3.68 \times 10^{4}, 3.68 \times 10^{4}$ & $3.03 \times 10^{8}, 3.03 \times 10^{8}$ \\
$\mathrm{Ca}$ & $3.70 \times 10^{6}, 2.81 \times 10^{6}$ & $1.36 \times 10^{6}, 1.04 \times 10^{6}$ & \\
$\mathrm{Ti}$ & $3.63 \times 10^{4}, 3.63 \times 10^{4}$ & $2.03 \times 10^{4}, 2.03 \times 10^{4}$ & \\
$\mathrm{Cr}$ & $1.06 \times 10^{5}, 1.06 \times 10^{5}$ & $7.19 \times 10^{4}, 7.19 \times 10^{4}$ & \\
$\mathrm{Fe}$ & $2.28 \times 10^{6}, 3.35 \times 10^{6}$ & $3.26 \times 10^{5}, 4.78 \times 10^{5}$ & \\
$\mathrm{Zn}$ & $8.24 \times 10^{5}, 8.24 \times 10^{5}$ & $4.27 \times 10^{5}, 4.27 \times 10^{5}$ & \\
\hline
\end{tabular}

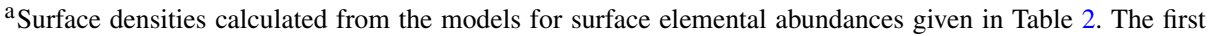
density provided is based on the additive model for surface elemental composition; the second density cited is based on the multiplicative model (see Table 2)

${ }^{\mathrm{b}}$ From Table 3 of Wurz et al. (2010) 
The results from the Wurz et al. (2010) study predict that ion sputtering would be expected to remove high-sputtering-yield elements (such as $\mathrm{H}, \mathrm{O}$, and $\mathrm{Na}$ ) from the surface, producing a chemically reduced surface enhanced in low-sputtering-yield elements (such as $\mathrm{Fe}$ and $\mathrm{Ti}$ ). Because of the simultaneous activity of sputtering and vaporization, however, the low-sputter-yield atoms would also be removed from the surface, though less efficiently. At Mercury the effects of micrometeoroid bombardment and sputtering by ions should display different variations with latitude (see discussion below).

\subsubsection{Chemical Sputtering}

Chemical sputtering releases atoms and molecules from regolith grains during chemical reactions between implanted solar wind or magnetospheric ions (predominantly implanted protons) and regolith material. Chemical sputtering may produce and remove elemental sodium (Potter 1995), hydrogen, hydroxyl, and water (e.g., Crider and Vondrak 2003) from the surface to the exosphere (where those species can then be lost through photo-ionization, Jeans escape, or radiation pressure). Laboratory experiments have shown that proton bombardment of oxides readily forms hydroxyl (Gruen et al. 1976; Siskind et al. 1977; Zellner et al. 1996), which enables chemical sputtering of hydroxyl and water (Crider and Vondrak 2003). The chemical sputtering of hydrogen molecules $\left(\mathrm{H}_{2}\right)$ occurs through graincatalyzed reactions (Duley and Williams 1993). Johnson and Baragiola (1991) predicted that the sputtering yield of water by solar wind protons is $\sim 0.01$ molecule/ion for the Moon. The solar proton flux at Mercury (discussed in detail below) is an order of magnitude higher at the magnetoshock. Only portions of this flux reach the surface, implying a similar water production rate at Mercury.

Solar wind irradiation, in conjunction with micrometeoroid bombardment, can produce single-domain $\mathrm{nFFe}^{0}$ by the following reaction within the impact melt that later forms agglutinates and grain patinas:

$$
2 \mathrm{H}+\mathrm{FeO} \rightarrow \mathrm{Fe}^{0}+\mathrm{H}_{2} \mathrm{O} .
$$

Potter (1995) proposed an alternative chemical reaction for producing sodium and water by proton or chemical sputtering of sodium-bearing silicates through the following reaction:

$$
2 \mathrm{H}+\mathrm{Na}_{2} \mathrm{SiO}_{3} \rightarrow 2 \mathrm{Na}+\mathrm{SiO}_{2}+\mathrm{H}_{2} \mathrm{O} .
$$

This reaction produces twice as much sodium as water. Potter (1995) argued that Mercury's daytime surface temperatures are optimal for this reaction. The $\mathrm{OH}$ radical also serves to reduce any $\mathrm{Fe}^{2+}$ to $\mathrm{Fe}^{0}$, thereby removing $\mathrm{O}^{2-}$ from surface lattice sites (Huguenin 1976) and thus helping to produce $n \mathrm{pFe}^{0}$ in the uppermost layers of regolith grains. Rates for the production of $\mathrm{npFe}^{0}$ are not currently known, yet these rates are crucial for understanding the magnitude of the weathering process. There are also arguments that the solar wind irradiation produces $\mathrm{H}_{2}$ and not $\mathrm{H}_{2} \mathrm{O}$ (Starukhina 2006).

Although lunar samples are dominated by anhydrous minerals (Papike et al. 1991), observations of spectral reflectance have shown that hydroxyl and water are ubiquitous on the lunar surface. These signatures have a temporal variation indicative of production via solar wind interaction (Vilas et al. 2008; Clark 2009; Pieters et al. 2009; Sunshine et al. 2009). An upper limit to $\mathrm{H}_{2} \mathrm{O}$ production on the Moon has been calculated to be 500 molecules $/ \mathrm{cm}^{3}$ (Killen and Sarantos 2013). Because of the substantially higher temperatures on Mercury it is not clear if similar $\mathrm{OH}$ and $\mathrm{H}_{2} \mathrm{O}$ production can create sufficiently long-lived $\mathrm{OH}$ and $\mathrm{H}_{2} \mathrm{O}$ to produce such signatures. 
Fig. 6 Example of a simple, one-electron transition for the desorption of neutrals via PSD from $\mathrm{SiO}_{2}$. (a) The $\mathrm{Na}$ at the surface is ionic $\mathrm{Na}^{+}$; a solar photon excites an electron in the substrate, which neutralizes the $\mathrm{Na}^{+}$and converts it to $\mathrm{Na}^{0}$.

(b) This charge transfer puts the $\mathrm{Na}$ on a repulsive potential. The repulsive force allows the non-thermal desorption of $\mathrm{Na}$ from the surface. Figure from Madey et al. (2002)

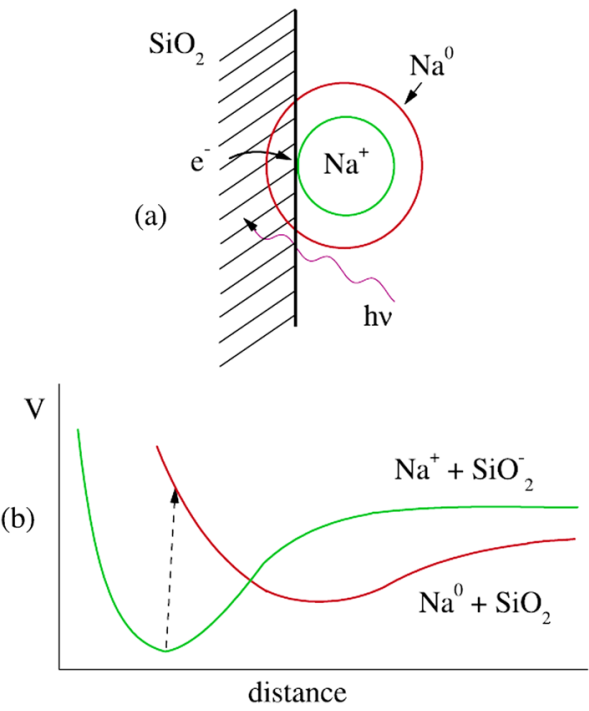

Sodium and water are not the only elements that can be chemically sputtered. Björkas et al. (2009) showed in the laboratory that Be can be chemically sputtered by deuterium bombardment, which has implications for other alkaline earth metals (such as $\mathrm{Mg}$ and $\mathrm{Ca}$ ). During MESSENGER's second flyby, Mg was detected in the exosphere for the first time (McClintock et al. 2009); Ca was discovered earlier in ground-based observations (Bida et al. 2000) and also observed by MESSENGER during the second and third flybys (McClintock et al. 2009). Although production of exospheric $\mathrm{Mg}$ and $\mathrm{Ca}$ via physical sputtering was examined by Wurz et al. (2010), there has been no detailed modeling of chemical sputtering as a plausible mechanism for producing exospheric $\mathrm{Mg}$ or $\mathrm{Ca}$.

\subsubsection{Desorption Induced by Electronic Transitions}

Desorption (of atoms) induced by electronic transitions is a surface physics phenomenon that can be triggered either by interactions with electrons (ESD) or photons (PSD). Both ESD and PSD lead to the desorption (removal from bulk composition to a gaseous or liquid phase) of atoms, molecules, and ions from a surface grain via electronic excitations. In the case of ESD, the electronic excitations are typically generated via the inelastic scattering of low-energy electrons $(\sim 4-50 \mathrm{eV})$ created by higher-energy incident radiation. The localization of these complicated multi-electron excitations at the surface occurs mostly at defects and leads to ESD. PSD generally refers to non-thermal desorption events that result from direct single-electron transitions to repulsive excited states involving either visible or ultraviolet photon radiation. PSD can also involve higher-energy photons, such as X-rays, which penetrate to greater depths in the regolith. In the case of X-ray interactions, PSD involves core-level atomic transitions.

The abundance of neutrals versus ions desorbed by DIET depends on the incident energy and chemical state of the surface grain material. For example, neutrals are preferentially desorbed relative to ions by several orders of magnitude for adsorbates on metallic surfaces and materials with lower-energy (sub-band-gap) excitations. For materials subjectable to Auger decay the ion yields become more appreciable, such as for oxides (i.e., wide band-gap materials) and mineral surfaces. (Auger decay is a two-electron process whereby a bound 
Intra-atomic Decay

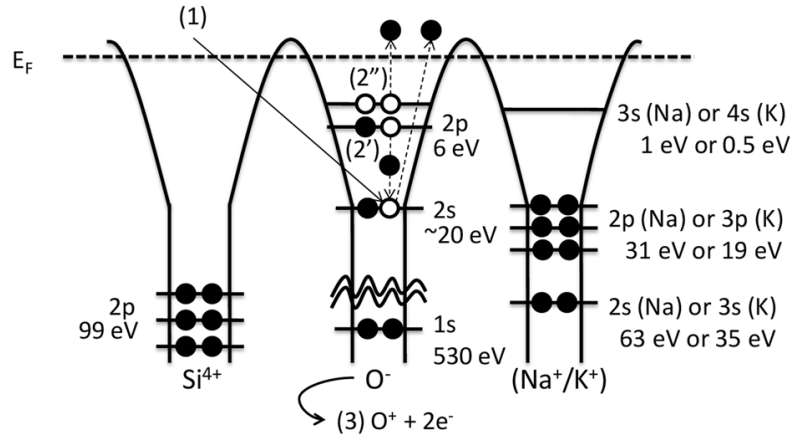

Fig. 7 Auger mechanism for electron-beam-induced desorption of $\mathrm{O}^{+}, \mathrm{Na}^{+}, \mathrm{K}^{+}$, and $\mathrm{Si}^{+}$ions (energies are not drawn to scale). This schematic shows intra-atomic decay of an initial hole in the 2 s level of $\mathrm{O}^{-}$ defects within the silicate surface. Auger decay reverses the Madelung potential and results in the formation of $\mathrm{O}^{+}$and Coulomb explosions. Auger processes can also involve holes and electrons correlated with the $\mathrm{Si}$ and $\mathrm{Na}$. In these cases, inter-atomic Auger decay results in similar Coulomb explosions. Figure from McLain et al. (2011), who provide additional details

electron transitions from a less bound shell to a vacant, more tightly bound shell, releasing energy that is transferred to a second bound electron, which escapes the atom.) A simple one-electron transition for desorption of neutrals via PSD is depicted in Fig. 6 (ESD behaves similarly), and Fig. 7 shows a more complicated multi-electron process involving Auger decay and Coulomb explosions. (Coulomb explosions are a process by which a high-velocity molecule strikes a solid and the electrons binding the molecule are rapidly removed via violent collisions with the electrons in the solid, thus producing a plasma of charged atomic constituents separated by their mutual Coulomb repulsion).

A key issue for DIET is "localization" or self-trapping of energy, which occurs best at defects, so DIET is highly sensitive to defect density. Defect sites have missing atoms and therefore have either deficient or excess charge. The differential charge distribution and reduced repulsion energy lead to the localization of an excitation (for example, excitations move efficiently in well-structured crystalline materials). The energy flow is stopped once defects are encountered, thus localizing and trapping the energy, leading to bond breaking and desorption. Amorphous and irradiated samples (in which lattice defects are more abundant) typical of regolith materials will thus tend to have higher DIET yields than singlecrystal, defect-free materials. The magnitude of this difference will depend on the density and distribution of lattice defects.

DIET processes relevant to Mercury have been evaluated through laboratory simulations and comparisons with measurements of the exospheres of both the Moon and Mercury. ESD and PSD both vary with solar activity, surface temperature, solar distance, and the physical characteristics of the regolith, such as porosity, which affects how desorbed materials stick to adjacent surface grains. The efficiency of ESD is affected by the interactions between the solar wind electrons and Mercury's intrinsic magnetic field (Sect. 3.4).

PSD may be the dominant mechanism producing Mercury's sodium exosphere (Burger et al. 2010; Wurz et al. 2010; Mouawad et al. 2011). Burger et al. (2010) argued that ion sputtering did not markedly contribute to exospheric sodium during MESSENGER's first two flybys, but that ion bombardment did contribute to the PSD process at high latitudes by enhancing diffusion. PSD, therefore, is a key process modifying the dayside regolith. DIET processes not only deplete minerals of alkali content, but they can remove most atoms 
and molecules from terminal sites within the lattice structure. In their comparisons of exospheric yields for different source processes, Wurz et al. (2010) showed that PSD has the potential to remove $\mathrm{Na}$ or $\mathrm{K}$ from Mercury's surface at rates three to four orders of magnitude higher than micrometeoroid impact vaporization or physical sputtering. ESD and PSD also remove both oxygen and hydrogen, leaving behind metals such as $\mathrm{Fe}$ and $\mathrm{Mg}$, sometimes in a reduced state. Neutral species are the dominant ejecta via PSD (Killen and Ip 1999). Refractory species are not affected by PSD (Killen et al. 2007).

Modeling by Lammer et al. (2003) has shown latitudinal and orbital dependencies of PSD yields. Sodium yields are greatest at equatorial latitudes during perihelion and are three times lower at the equator at aphelion (Killen et al. 2007). The removal of alkalis from the surface by PSD is tied to the solar UV flux. The UV flux can increase by up to a factor of 100 from quiet-Sun to active solar periods or during solar flares (Killen et al. 2007), indicating that the effectiveness of this process is highly time dependent. The solar fluctuation in the near-UV is not this large, so desorbtion by PSD in this energy region $(\sim 4 \mathrm{eV})$ will be less time variable.

\subsubsection{Thermal Desorption}

Thermal desorption is the removal of adsorbed atoms from a surface via heating. The effectiveness of this process is influenced by volume diffusion from grain interiors to surfaces, surface diffusion between sites with different desorption energies, and electronic excitation and de-excitation (Killen et al. 2007). The rate of thermal desorption is governed by diffusion, grain size, and grain lifetime at the regolith surface (Killen et al. 2004, 2007). For example, smaller grains diffuse atoms to their surface more quickly than larger grains and are depleted more rapidly in volatiles via thermal desorption (Killen et al. 2004). However, small grains also serve as a more efficient repository for adsorption of volatiles due to their larger surface-to-volume ratio.

Leblanc and Johnson (2003) argued that thermal desorption rapidly depletes most of Mercury's sunlit surface of adsorbed atoms, but they did not consider resupply by diffusion in their calculations. Over geologic timescales, equatorial regions are depleted in adsorbed alkalis (or other species such as $\mathrm{H}$ and $\mathrm{OH}$ ), which migrate either poleward (Killen and Morgan 1993; Killen et al. 2007) or to temporary nightside cold traps (Leblanc and Johnson 2003), thus replenishing the surface in those locations. Also, some of the desorbed species (including alkalis) are ionized and removed from the system by the solar wind. As with all processes, thermal desorption works in conjunction with the other processes acting within Mercury's surface-exosphere-environment system. Thermal desorption is enhanced by lattice defects created during solar wind irradiation, since these defects facilitate diffusion from grain interiors. Regolith gardening governs grain exposure time at the surface, thus controlling the volatile depletion rate. As discussed above, the gardening of Mercury's surface is more efficient than that at the Moon, but no quantitative comparisons of grain surface lifetimes have been published.

\subsection{Deposition of Volatiles within the Regolith}

The exosphere can be regarded as a transition zone between the upper regolith and loss of material to space. The cumulative effects of the weathering processes can be understood by examining lunar samples and measures of the lunar exosphere. Lunar regolith grains reveal evidence for volatile loss through compositional gradients and isotopic ratios. For example, the volatile metal mercury $(\mathrm{Hg})$ was measured in Apollo 11, 12, 14, and 15 samples and 
Luna 16 core and trench samples (e.g., Jovanovic and Reed 1972, 1979a, 1979b). The Hg compositional gradients indicate diffusive loss from the soil and repeated resurfacing events. The sequestration of $\mathrm{Hg}$ as a polar condensate was predicted to be as high as $0.25 \mathrm{wt} \%$ in cold traps (Reed 1999). The cold-trapping of $\mathrm{Hg}$ was confirmed by the observations of $\mathrm{Hg}$ in the impact vapor formed by the impact of the Lunar Crater Observation and Sensing Satellite (LCROSS) (Gladstone et al. 2010). Quantifying the amount of $\mathrm{Hg}$ in the polar traps constrains models of cold-trapping efficiencies at the lunar poles. The mass mixing ratio of $\mathrm{Hg}$ in the vapor plume measured by the Lyman Alpha Mapping Project (LAMP) instrument on the Lunar Reconnaissance Orbiter following the LCROSS impact into Cabeus crater was reported to be $1.2 \%$ (Gladstone et al. 2010), five times the predicted value for permanently shaded lunar soils (Reed 1999). The discrepancy may indicate that the cold-trap area is smaller than the $3700 \mathrm{~km}^{2}$ assumed by Reed (1999), or that the most volatile species were preferentially measured.

This evidence for lunar cold-trapping has implications for cold-trapping of volatiles on Mercury. Many of the processes that remove volatiles (including alkalis) have been argued to be more efficient at Mercury than at the Moon, thus placing more material in motion. Once these materials are removed from the surface to the exosphere, two paths are possible for their final deposition. Neutral species will either be redirected back to the surface or lost through ionization or photo-dissociation. If redirected to the surface, they either are deposited near their ejection region or migrate poleward to be trapped in permanent or semipermanent cold traps. Ions can be recycled to Mercury's surface by gyromotion about magnetic field lines (a similar mechanism operates at the Moon, where pick-up ions gyrate about interplanetary magnetic field lines, resulting in recycling of some of these ions to the surface) or removed from the system by being picked up in the solar wind and lost downstream (e.g., Hartle and Killen 2006). A steady state between removal from and redeposition onto the surface depends on process rates, regolith porosity (mean free path of ejected material), and gardening rates.

Measurements of elemental abundances of such volatile species as sulfur (S), sodium (Na), and potassium (K) from the MESSENGER spacecraft's Gamma-Ray Spectrometer (GRS) and X-Ray Spectrometer (XRS) during orbital operations indicate that Mercury's surface may not be volatile depleted compared with the other terrestrial planets (Nittler et al. 2011; Peplowski et al. 2011, 2012; Evans et al. 2012; Weider et al. 2012). Alternatively, the process of partitioning silicates from an iron core in a low-oxygen-fugacity environment could produce misleadingly high $\mathrm{K} / \mathrm{Th}$ ratios (as an indicator of a planet's volatile inventory) even if Mercury is actually volatile poor (McCubbin et al. 2012), though this argument does not account for Mercury's high Na and S abundances. The detection of these volatile species within Mercury's regolith argues not only for their presence within the mineral structure of the regolith grains, but also for efficient mechanisms for trapping these volatile materials within the regolith from the exosphere. Lunar soils show an increased concentration of volatile elements with decreasing soil size fraction, indicating that volatiles may be trapped on grain surfaces so that enrichment in the smaller size fraction may be due to their larger surface-to-volume ratio (Krähenbühl et al. 1977; McKay et al. 1991). With the increased micrometeoroid flux and impact velocity (Cintala 1992; Borin et al. 2009) on Mercury compared with the Moon, Mercury's regolith is arguably finer grained (larger portion $<100 \mu \mathrm{m}$ size fraction) and thus a potentially more efficient sink for volatiles than its lunar counterpart. Additionally, Mercury's surface is more radiation damaged than the lunar surface (as discussed in Sect. 3), creating a higher abundance of lattice defects within regolith grains, which act as efficient adsorbers of volatiles, regardless of grain size. The more rapid gardening or overturn rates indicate that these volatiles 
Fig. 8 Map of the abundance of $\mathrm{K}$ in Mercury's northern hemisphere derived from MESSENGER gamma-ray measurements (from Peplowski et al. 2012). Outlines of the northern smooth plains (black line) and Caloris basin (gray line) are also shown

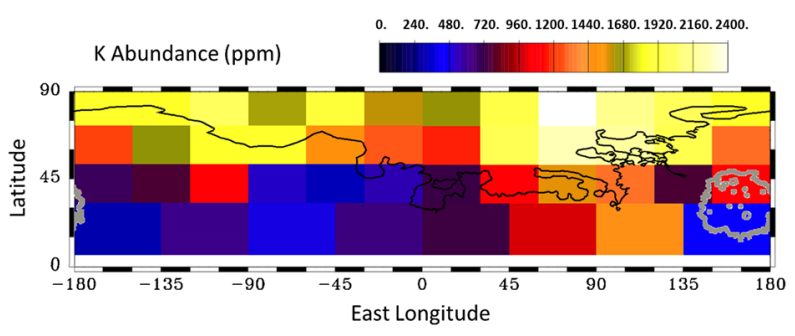

not only can be trapped more efficiently, but can be brought to depth more efficiently. The general agreement between abundance measurements for aluminum (Al), S, and calcium (Ca) from the XRS and GRS, which sample the regolith to different depths, indicates that on average Mercury's regolith is homogeneous to depths of tens of centimeters (Evans et al. 2012), supporting this interpretation.

Cold-trapping of volatiles at Mercury's poles, similar to that on the Moon, is another indicator of volatile retention within the regolith. Species that have been speculated to be cold-trapped at the poles include water (e.g., Harmon and Slade 1992; Paige et al. 1992; Slade et al. 1992) and sulfur (Sprague et al. 1995). Orbital measurements from MESSENGER's Neutron Spectrometer (NS) and Mercury Laser Altimeter (MLA), in addition to thermal modeling with topographic variations measured by MLA, show the presence of water ice in the permanently shadowed regions of the north pole (Lawrence et al. 2013; Neumann et al. 2013; Paige et al. 2013). The NS measurements indicate buried ice deposits within the permanently shadowed regions that are at least tens of centimeters thick and generally covered by a surficial layer lower in $\mathrm{H}$ that is 10 to $30 \mathrm{~cm}$ thick (Lawrence et al. 2013). These NS measurements are best represented by a model in which a pure water ice layer is covered by a regolith layer containing less than $25 \mathrm{wt} \%$ water-equivalent hydrogen (Lawrence et al. 2013). MLA reflectance observations and thermal models at higher spatial resolution indicate both exposed and covered regions of water ice (Neumann et al. 2013; Paige et al. 2013).

Evidence for possible thermal control of elemental abundances is seen in the spatial variations in $\mathrm{K}$ abundance measured by the GRS (Peplowski et al. 2012). Figure 8, from Peplowski et al. (2012), displays the K abundance, which ranges from $300 \mathrm{ppm}$ at low latitudes to $2400 \mathrm{ppm}$ over the northern hemisphere. A portion of the spatial variations may be the result of differences in $\mathrm{K}$ abundance among geological units, i.e., the northern smooth plains appear to be generally higher in $\mathrm{K}$ than the adjacent regions (Fig. 8). Comparisons of $\mathrm{K}$ abundance with the maximum surface temperature at $7 \mathrm{~cm}$ depth (Fig. 9) show that in regions for which the maximum surface temperature exceeds $350 \mathrm{~K}$, the two quantities are anticorrelated, indicating that they are related (Peplowski et al. 2012). A similar relationship has been postulated for $\mathrm{Na}$, on the grounds that GRS measurements of the latitude dependence of the $\mathrm{Na} / \mathrm{Si}$ abundance ratio shows a north polar enhancement that is consistent with mobilization of $\mathrm{Na}$ from Mercury's hot poles and redeposition in the cooler polar regions (Peplowski et al. 2014). Thus although $\mathrm{Na}$ is present globally within the crystalline structure of one or more host minerals, some of the enhancement at high latitudes can be ascribed to adsorption or redeposition (as in the vapor-coated patinas) in colder regions.

\subsection{Laboratory Simulations}

Laboratory simulations of space weathering processes have been conducted to examine micrometeoroid and ion bombardment, DIET processes, and thermal desorption effects on mineral and mineral powders. 
Fig. 9 (Top) Maximum calculated temperature at $7 \mathrm{~cm}$ depth on Mercury (D.A. Paige, personal communication, 2012) at a spatial resolution equivalent to the $\mathrm{K}$ abundance map shown in Fig. 8, taken from Peplowski et al. (2012). The temperatures are derived from the formalism of Vasavada et al. (1999), which does not include surface topography. Regions in white are outside of the GRS coverage for the K abundance. (Bottom) Plot of measured $\mathrm{K}$ abundance (from Fig. 8) versus maximum temperature at $7 \mathrm{~cm}$ depth from Peplowski et al. (2012). A linear fit to the data at all temperature ranges (red) is compared with a linear fit to data only at temperatures above $350 \mathrm{~K}$ (purple). The correlation coefficient for both fits indicates a strong anticorrelation between the $\mathrm{K}$ abundance and temperature. See Peplowski et al. (2012) for additional details
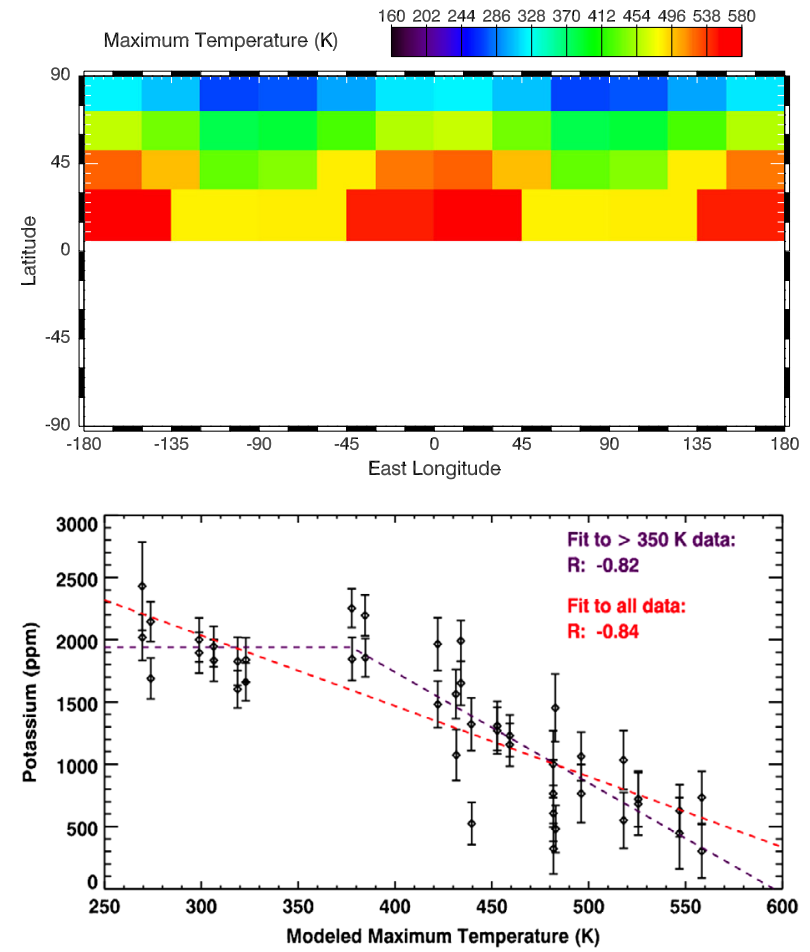

The micrometeoroid bombardment of asteroid surfaces has been simulated with pulsed lasers acting on powdered silicates (e.g., orthopyroxene, clinopyroxene, and olivine) (Moroz et al. 1996; Yamada et al. 1999; Sasaki et al. 2001, 2003; Hiroi and Sasaki 2001; Brunetto et al. 2006a; Loeffler et al. 2008a, 2008b). These experiments address the effect on spectra from UV to NIR wavelengths of vapor-deposited $n \mathrm{pFe}^{0}$ and provide clues to spaceweathering rates. However, there are issues concerning the fidelity with which laser pulses simulate micrometeoroid bombardment, especially regarding the depth of penetration. Laser irradiation effects depend on the energy flux of the single pulse, pulse duration, and wavelength of the beam (e.g., Chrisey and Hubler 1994). Fluence determines which of two energy regimes the experiment is simulating. At low flux, laser irradiation induces mainly thermal and chemical effects. At higher flux, laser-light intensity can induce vaporization, depending on target properties and laser parameters. With silicate samples, which have strongto-medium laser absorption, the threshold fluence for vaporization for nanosecond-pulsed lasers is typically between 0.5 and $2 \mathrm{~J} / \mathrm{cm}^{2}$ per pulse (Bäuerle 2000).

The first laser space weathering experiments performed on ordinary chondrites used microsecond-pulsed laser irradiation to redden and darken reflectance spectra (Moroz et al. 1996). The pulse duration in these experiments was much longer than micrometeoroid (1-10 $\mu \mathrm{m}$ size) impact timescales, so the observed spectral changes may have resulted from glass formation (Sasaki et al. 2001). Shorter pulse durations (6-8 ns) were employed by Yamada et al. (1999), Sasaki et al. (2001, 2003), and Hiroi and Sasaki (2001), who used a nanosecond-pulsed Nd:YAG infrared laser (1064 nm wavelength) on pressed silicate powder pellets. The samples showed progressive darkening and reddening of silicate spectra from UV to NIR wavelengths with increasing shot number, interpreted as contributing to the formation of coatings enriched in vapor-deposited $n p F e^{0}$ (Sasaki et al. 2001). 
Brunetto et al. (2006a) noted an issue with infrared lasers: changes in target material optical properties were the result of surface damage and stresses induced by thermalization from the incident infrared beam. In contrast, an ultraviolet laser causes direct molecular bond breaking and ablation to occur, and rapid heating and cooling rates produce less surface damage to materials. Experiments by Brunetto et al. (2006a, 2006b) with a nanosecondpulsed UV (193 and $248 \mathrm{~nm}$ wavelength) excimer laser on pressed silicate powder pellets produced, for an energy dose comparable to that produced by micrometeoroid bombardment over a time period of $10^{8} \mathrm{y}$ at $1 \mathrm{AU}$, higher spectral slopes than those of Sasaki et al. (2001) by a factor of $\sim 50 \%$, suggesting that UV excimer lasers are more efficient than infrared lasers at reddening silicate samples and may be a more accurate analogue to micrometeoroid bombardment on planetary bodies.

Space weathering effects depend on surface composition. Laboratory simulations of micrometeoroid bombardment, conducted with laser radiation techniques as described above, in low-iron materials $(<10 \mathrm{wt} \% \mathrm{FeO})$, show variations in spectral alterations with composition (Sasaki et al. 2002; Marchi et al. 2005b). For example, Sasaki et al. (2002) irradiated samples of low-iron olivine ( $8.97 \mathrm{wt} \% \mathrm{FeO})$ and pyroxene $(9.88 \mathrm{wt} \% \mathrm{FeO})$ and found that spectral changes were more pronounced in the olivine samples. Similarly, Marchi et al. (2005b) irradiated iron-poor olivine and orthopyroxene to examine ion-sputtering-induced spectral changes and also found larger spectral changes in the olivine samples, showing that this compositional dependence affects both micrometeoroid impact and physical sputtering processes.

Currently it is not clear whether UV excimer or near-infrared laser irradiation more closely simulates micrometeoroid space weathering. It is possible that one laser type replicates Mercury's micrometeoroid process, whereas the other matches micrometeoroid weathering on asteroids, as melting will vary with impact parameters. For instance, near-infrared laser experiments that produce relatively high degrees of melting by thermalization could be more relevant to micrometeoroid weathering on Mercury, whereas UV laser simulation, with its lower melt production, might be more relevant for asteroids.

Laboratory simulations of solar wind irradiation of silicates have been conducted in an attempt to explain space weathering on asteroids. Solar wind irradiation processes have been thought to dominate the alteration of asteroid surfaces over micrometeoroid bombardment due to the lower micrometeoroid impact velocities (especially on near-Earth asteroids) and the lower flux of interplanetary dust (both are relevant on main-belt asteroids) on asteroids compared with the Moon. In addition, the observed inverse correlation of the degree of spectral reddening in asteroid observations with solar distance $r$ (flux decreases as $1 / r^{2}$ ) has led to the suggestion that solar wind irradiation is the primary mechanism for weathering asteroid surfaces (Marchi et al. 2006), though this connection can be argued as tenuous due to scatter in the supporting data and variations in the average composition of asteroids with distance from the Sun (Loeffler et al. 2009). More convincing evidence of the role of solar wind irradiation is seen in the Itokawa samples returned by the Hayabusa spacecraft. Noguchi et al. (2014) showed surface regions on the sample grains that contain $n \mathrm{nFe}^{0}$ adjacent to areas depleted in Fe content, suggesting the in situ formation of the $\mathrm{npFe}^{0}$ via irradiation as opposed to vapor deposition.

Bidirectional reflectance measurements from 0.3 to $2.67 \mu \mathrm{m}$ of an ordinary chondrite (Epinal H5) irradiated with $60 \mathrm{keV} \mathrm{Ar}^{2+}$ ions over a series of different ion fluences show progressive reddening and darkening of the reflectance properties comparable to the weathering trends postulated for asteroids (Strazzulla et al. 2005). Irradiation studies of bulk silicate samples, including olivine, pyroxene, and serpentine, conducted with a variety of ion species $\left(\mathrm{H}^{+}, \mathrm{He}^{+}, \mathrm{Ar}^{+}, \mathrm{Ar}^{2+}\right)$ at different energies $(60$ to $400 \mathrm{keV})$ were conducted by Brunetto and 
Strazzulla (2005) to examine the physical mechanism that produces the spectral alterations of this weathering process. They examined the reflectance properties (between 0.25 and $2.7 \mu \mathrm{m}$ ) of their samples and found correlations between reddening, darkening, and band area reduction with the formation of nuclear displacements within the samples determined through Raman spectroscopy. [The samples of Brunetto and Strazzulla (2005) have the $1 \mu \mathrm{m}$ (band I) feature of olivine and pyroxene in addition to the $2 \mu \mathrm{m}$ (band II) pyroxene feature.] Brunetto and Strazzulla (2005) concluded that the reddening and darkening can be attributed to the amorphization of the very upper layers $(<1 \mu \mathrm{m})$ of the sample grains. The decrease in band areas is due to changes in the optical properties of this same upper layer (Brunetto and Strazzulla 2005). Loeffler et al. (2009) examined both the reflectance and structural changes produced by the irradiation of olivine by $4 \mathrm{keV} \mathrm{He}^{+}$ions. The results produced spectral changes similar to those seen in the laser ablation experiments mimicking micrometeoroid bombardment (Brunetto et al. 2007) and previous ion irradiation experiments mimicking solar wind radiation (Strazzulla et al. 2005; Brunetto et al. 2006b). From X-ray photoelectron spectroscopy (XPS), Loeffler et al. (2009) demonstrated that the reddening is clearly correlated with an increase in metallic iron. In their experiments Loeffler et al. (2009) observed spectral changes only at high fluences, and they concluded that chemical reduction and not amorphization was responsible for the reflectance changes they measured. Since the spectral change observed was reddening and not darkening of the spectrum, Loeffler et al. (2009) argued that the metallic iron grains detected in the XPS measurements were very small, consistent with laboratory studies of the effects of different size $\mathrm{npFe}^{0}$ on spectral properties (Noble et al. 2007). The reddening observed in the olivine from the experiment of Loeffler et al. (2009) occurred predominantly below $1.07 \mu \mathrm{m}$ wavelength. In addition to reddening, Loeffler et al. (2009) observed decreases in band areas, but the decreases were larger in the band I ( $1 \mu \mathrm{m})$ absorption feature than in the band II $(2 \mu \mathrm{m})$ absorption feature. This type of band reduction is similar to the space weathering effects observed in lunar and asteroidal spectra. The properties of these bands are used to determine Fe content within the olivine and pyroxene minerals, and thus understanding space-weathering effects on the properties of these bands is important for accurately deriving iron contents of surface minerals.

Another aspect studied by Loeffler et al. (2009) was the variation in the spectral and chemical changes between olivine slabs and powders derived from these slabs. The creation of $\mathrm{nFFe}^{0}$ was $\sim 2$ times slower in the powders than on the flat sections (Loeffler et al. 2009). The slower rate of $n \mathrm{pFe}^{0}$ production in the powders was ascribed to greater redeposition in the powder samples (Loeffler et al. 2009). As sputtered material is redeposited, it is partially oxidized (Loeffler et al. 2009), a phenomenon that has been observed in sputter deposition of oxides in a vacuum (Mauvernay et al. 2007; Loeffler et al. 2009). The production of npFe ${ }^{0}$ by ion irradiation does not require redeposition of Fe (Dukes et al. 1999; Davoisne et al. 2008; Loeffler et al. 2009). Loeffler et al. (2009) estimated that approximately two-thirds of the ejecta produced in the radiation of powders is redeposited (and partially oxidized), a value similar to the $70 \%$ redeposition predicted by the modeling studies of Cassidy and Johnson (2005). These results imply that the production of Fe metal is slower in a regolith than on solid un-comminuted surfaces. This conclusion implies that factors that enhance redeposition (such as greater surface gravitational acceleration or less compact or larger fine-grained regolith components) will inhibit $\mathrm{nFFe}^{0}$ production.

The rates of weathering by solar wind ions versus micrometeoroid bombardment are key to establishing the dominant space weathering process for different bodies in the Solar System. On the basis of their experimental results Loeffler et al. (2009) derived a characteristic time for weathering of a surface exposed to the solar wind of $\sim 5000 \mathrm{y}$, but this characteristic time does not account for interactions between the effects of ion and micrometeoroid 
bombardment, such as gardening and comminution, which will increase this characteristic time. Loeffler et al. (2009) estimated that reddening effects on reflectance spectra of asteroids caused by solar wind interactions saturate some two orders of magnitude faster than for estimated rates of micrometeoroid bombardment. The irradiation experiments of Strazzulla et al. (2005) on the ordinary chondrite sample correspond to solar wind exposure times of $10^{4}-10^{6} \mathrm{y}$ for the spectral changes they observed. This value is consistent with the results of Brunetto and Strazzulla (2005), who estimated that the ion irradiation mechanism operates on timescales of $<10^{6} \mathrm{y}$.

Laboratory analyses of DIET processes have typically focused individually on ESD or PSD, though the two processes have many similarities. Yakshinskiy and Madey (2000) showed that both ESD and PSD have threshold energies of $\sim 4 \mathrm{eV}$ and the desorbing atoms are "hot" with suprathermal velocities (though not as hot as vapor or ion sputter products). For example, PSD-desorbed sodium is suprathermal with a peak speed of $\sim 900 \mathrm{~m} / \mathrm{s}$ and a velocity distribution best described by a Weibull function (Yakshinskiy and Madey 2004), such as

$$
f(E)=\beta(1+\beta) \frac{E U^{\beta}}{(E+U)^{2+\beta}},
$$

where $U$ is the characteristic energy related to the surface binding energy, $\beta$ is a shape parameter, and $E$ is the energy of the expelled atom. For ESD the values of $U$ and $\beta$ are $0.052 \mathrm{eV}$ and 0.7 , respectively.

Simulations of ESD have been conducted primarily on amorphous $\mathrm{SiO}_{2}$ films (Yakshinskiy and Madey 2000, 2003, 2004, 2005), Na- and K-bearing glasses (McLain et al. 2011), and lunar basalts over which a Na monolayer has been deposited (Yakshinskiy and Madey 2003, 2004, 2005). These experiments have shown temperature dependencies of DIET yields that factor into removal rate differences for atoms and molecules between the Moon and Mercury as a result of this process. DIET desorption of Na was shown to increase tenfold from 100 to $470 \mathrm{~K}$ (Yakshinskiy and Madey 2004). Yakshinskiy and Madey (2005) observed a temperature dependence of ESD-desorbed $\mathrm{Na}$ from lunar basalt that reverses with the heating and cooling of the lunar sample. Experimental ion $\left(\mathrm{H}^{+}, \mathrm{H}_{2}^{+}, \mathrm{O}^{+}, \mathrm{H}_{3} \mathrm{O}^{+}\right.$, $\mathrm{Na}^{+}, \mathrm{K}^{+}$, and $\left(\mathrm{O}_{2}^{+}\right)$yields display slight temperature dependencies between 150 and $350 \mathrm{~K}$, but these ion yields show linear increases with temperature between 350 and $550 \mathrm{~K}$ (McLain et al. 2011). Above $400 \mathrm{~K}$ the ion yields from the samples show temperature dependencies that are reversible and reproducible (McLain et al. 2011). These temperature dependencies have been attributed to thermally induced changes in grain-surface bonding sites (Yakshinskiy and Madey 2004). Changes in density and the location of defect sites, at which thermal changes induce changes in local atomic coordination site (position within the crystal lattice relative to nearby atoms), enhance the ESD process and can explain the reversible temperature dependence seen in experimental desorption yields (Yakshinskiy and Madey 2004; McLain et al. 2011). This result is also consistent with lattice expansion, increased hole localization within surface defects, and the diffusion of defects or vacancies to the grain surface (Chen et al. 2005; McLain et al. 2011).

Desorption yields from films, however, differ from yields within regolith due to increased sticking probabilities within a porous, multi-particle medium. Measurements of $\mathrm{Na}$ and $\mathrm{K}$ sticking probabilities show that $\mathrm{K}$ has a nearly constant sticking probability over $100-500 \mathrm{~K}$, whereas Na displays a decreasing sticking probability with increasing temperature over this temperature range (Yakshinskiy and Madey 2005). DIET desorption yields are therefore species dependent. Table 4, from the experimental results of McLain et al. (2011), shows threshold energies for the desorption of several ions from Na- and K-bearing glasses. 
Table 4 Desorption threshold energies for Na- and K-bearing glasses

\begin{tabular}{|c|c|c|c|c|c|c|c|}
\hline Species & $\mathrm{H}^{+}$ & $\mathrm{H}_{2}^{+}$ & $\mathrm{O}^{+}$ & $\mathrm{H}_{3} \mathrm{O}^{+}$ & $\mathrm{Na}^{+}$ & $\mathrm{K}^{+}$ & $\mathrm{O}_{2}^{+}$ \\
\hline Threshold energy (eV) & $25 \pm 2$ & $40 \pm 2$ & $30 \pm 2$ & $40 \pm 2$ & $30 \pm 2$ & $30 \pm 2$ & $90 \pm 2$ \\
\hline
\end{tabular}

ESD experiment results have been combined with electron $\left(\mathrm{e}^{-}\right)$precipitation simulations to predict ion outflow from Mercury's surface. For solar wind conditions matching those observed during MESSENGER's first two Mercury flybys, and integrating over the entire planet, simulations indicate that $\sim 10^{26} \mathrm{e}^{-} / \mathrm{s}$ impact Mercury's surface (Schriver et al. 2011a). Combination with laboratory ESD ion yields of $10^{-3}-10^{-5}$ ions/ $\mathrm{e}^{-}$(with the value dependent on the impinging electron energy over the interval $200 \mathrm{eV}-2 \mathrm{keV}$ ) gives a value of $10^{21}-10^{23}$ ions/s (of all species combined) emitted from Mercury (McLain et al. 2011; Schriver et al. 2011a). This range is comparable with estimates of the sputtered outflow yields of neutral $\mathrm{Na}$ alone of between $6.0 \times 10^{21}$ and $3.8 \times 10^{24} \mathrm{~s}^{-1}$ (Killen et al. 2004). Though the comparison here is between ions and neutrals, ions sputtered from the lunar or Mercury surface are quickly neutralized.

Thermal desorption has been studied with $\mathrm{SiO}_{2}$ (Madey et al. 1998) and $\mathrm{Al}_{2} \mathrm{O}_{3}$ (Shao and Paul 1993) films coated with mono-layers of sodium. Laboratory measurements show that desorption yields are related to both temperature and sodium layer thickness (Madey et al. 1998). Fractional mono-layers desorb at appreciable rates for temperatures greater than 500-600 K for $\mathrm{SiO}_{2}$, whereas multi-layers desorb at much lower temperatures with a peak near 350 K (Madey et al. 1998; Killen and Ip 1999). The desorption temperature from $\mathrm{Al}_{2} \mathrm{O}_{3}$ surfaces ranges from 300 to $400 \mathrm{~K}$ for both fractional and multi-layers of sodium (Shao and Paul 1993; Killen and Ip 1999).

Rates of thermal desorption from metal oxide surfaces depend on the composition of the minerals and the presence of other atoms in low concentrations (Killen et al. 2007). For example, the thermal desorption energy for potassium from the same type of mineral can change from 0.83 to $2.35 \mathrm{eV}$ by the inclusion of $2 \mathrm{wt} \% \mathrm{Mn}$ (Kotarba et al. 2004). Studies suggest that the desorption energy of alkali atoms from oxide minerals is $\sim 2 \mathrm{eV}$ or greater (Holmlid 2006). These experiments indicate that thermal desorption rates will vary among the Moon, Mercury, and asteroids on the basis of compositional differences in addition to temperature.

\subsection{Synthesis of Space Weathering Processes}

Theoretical, laboratory, and lunar sample studies along with remote observations help us to assess effects of the various exosphere-environment-surface processes on Mercury. Micrometeoroid bombardment involves gardening and the production of melt and vapor and exhibits compositionally dependent processing. Solar radiation processes are influenced by many factors (e.g., grain diffusion rates, solar wind and interplanetary magnetic field characteristics, surface flux), and their long-term effects depend on regolith parameters controlled by impacts (such as gardening rates, regolith grain size, and porosity). Here we briefly integrate the discussion of processes in the previous sections, with a particular focus on conditions on Mercury.

Theoretical studies (Cintala 1992; Borin et al. 2009) tied to impact experiments suggest that Mercury's regolith is overturned more quickly than the lunar regolith. Regolith grains on Mercury are exposed at the surface for less time but more often, because impact excavation volumes are slightly elevated and impactor flux is much higher on Mercury. The 
accumulated time a grain spends at the surface, exposed to the space environment, remains unknown. Accumulated exposure time affects all surface maturation process rates. For example, asteroids (especially smaller ones) have a net loss of material due to impacts, so that processed regolith is relatively quickly replaced by fresh substrate material. On the Moon and Mercury, however, the same surficial regolith material is repeatedly bombarded, churned, and subjected to space weathering processes, whereas larger impacts excavate fresh material only sporadically.

A given impact on Mercury is expected to produce an order of magnitude or more impact melt and vapor than its equivalent on the Moon (Cintala 1992; Borin et al. 2009), implying that more impact glasses and agglutinates should be present in Mercury's regolith. Conversely, asteroids should have much less melt and vapor products than the Moon or Mercury. Larger production of vapor might coat Mercury soil grains with thicker rims, though whether this effect occurs depends on surface residence time, which is currently unknown.

The rims of regolith grains, especially the outermost few mono-layers, are also processed by solar irradiation, depending on solar wind properties and solar flux (ions and photons) to the surface. We assume that solar wind composition and velocity are similar among Mercury, the Moon, and the main asteroid belt, but flux varies with solar distance and surface irradiation is affected by Mercury's magnetic field (see further discussion below). At this point we will assume that the solar wind can at times reach Mercury's surface, an assumption examined in the next section. Solar radiation processes include ion implantation, sputtering, DIET, PSD, and thermal desorption.

Ion implantation introduces ions into a grain's atomic lattice structure, creating lattice defects, which enhance diffusion from a grain's interior to its surface, causing a radiationdamaged, amorphous rim to develop on its surface. These effects contribute to a chemically reducing environment and production of $\mathrm{npFe}^{0}, \mathrm{OH}$, and $\mathrm{H}_{2} \mathrm{O}$ (if $\mathrm{O}, \mathrm{H}$, and $\mathrm{Fe}$ are present in the target mineral).

Sputtering processes (both physical and chemical) preferentially remove lighter species $(\mathrm{H}, \mathrm{O}$, and $\mathrm{OH})$ and thereby reduce the top mono-layers of surface grains. Laboratory experiments show that sputtering also removes alkali species. Sputtering, therefore, contributes to the creation of amorphous rims on exposed grain surfaces and depletion of the top monolayers of alkalis. Though much of the sputtered material can escape, some trapping of material by adjacent soil particles occurs. The effectiveness of this trapping is governed by surface temperature, regolith porosity, and regolith grain sizes and has been calculated to be as high as $70 \%$ (Loeffler et al. 2009). The rate of trapping will also increase as the surface gravitational acceleration increases, and thus will be more effective on large bodies such as Mercury than small asteroids such as Itokawa or Eros.

DIET processes operate in a manner similar to sputtering by removing elements from the top mono-layers of exposed surface grains. Laboratory studies indicate that thermal desorption and PSD are more efficient at removing volatiles (including alkalis) from warmer surfaces, which would imply that Mercury's surface should be more depleted in volatiles and alkalis than those of the Moon or main-belt asteroids. However, the detection of sulfur by MESSENGER's XRS (Nittler et al. 2011) and of sodium and potassium by MESSENGER's GRS (Peplowski et al. 2011; Evans et al. 2012) contradicts this prediction and moreover indicates that Mercury's surface material is inherently higher in sulfur than lunar or asteroidal surface materials. GRS observations (Rhodes et al. 2011; Evans et al. 2012) indicate that XRS-derived abundances extend to tens of centimeters in depth. The enhanced abundances of $\mathrm{Na}$ and $\mathrm{K}$ at the colder, higher latitudes compared with the warmer equatorial regions could be the result of temperature-enhanced removal at equatorial latitudes and preferential cold-trapping and adsorption at higher latitudes. Calculations 
by Killen and Sarantos (2013) suggest the high-latitude enhancement is not in the form of adsorbed $\mathrm{Na}$, but that the $\mathrm{Na}$ in this region is most likely within the regolith, diffused inward, and well mixed.

All processes discussed here are affected by diffusion rates and surface composition. Diffusion rates, in turn, depend on mineral composition, temperature, and solar wind exposure (radiation damage enhances diffusion rates). Diffusion rates at Mercury and the Moon differ because of different local thermal environments and precipitating plasma energies. Although the solar wind has the same mean energy at Mercury and the Moon, protons are accelerated when they cross Mercury's cusp region (Massetti et al. 2007). Typical solar wind particles impact the Moon with energies of $\sim 1 \mathrm{keV}$. At Mercury, impacting protons have energies of $\sim 3-7 \mathrm{keV}$. From lunar exospheric measurements, an energy dependence for Na diffusion is expected: when the Moon encounters terrestrial magnetospheric plasma-sheet ions $(\sim 3 \mathrm{keV})$, the sodium exosphere is enhanced over times when the Moon passes through the Earth's magnetosphere but misses the current sheet. If this effect is attributed to enhanced grain diffusion as a result of vacancies created by radiation from more energetic plasma (Sarantos et al. 2008, 2011), then, by extrapolation, diffusion should be expected to be more enhanced at Mercury because the plasma precipitating onto the surface is more energetic than solar wind plasma impacting the Moon.

Composition also affects the relative rates and efficiencies among processes. For example, comminution rates and depths depend on composition; a feldspathic surface breaks down more rapidly than a pyroxene-rich surface. This difference affects the regolith grainsize distribution and thus the mineral percentages in the spectrally dominant finer $(<45 \mu \mathrm{m})$ size fraction. The larger ratio of surface to volume for smaller grains both enhances diffusion rates from their interiors to their surfaces and enhances adsorption of atoms and molecules so that they are repositories for exospheric species. Thus the percentage of finer grains within a regolith influences its source and sink properties, though the balance is not well known.

Differential melting of material, which explains many lunar sample characteristics, is key to understanding melt and vapor contributions during impact processing. A mineraldependent preferential melting sequence (in the order glass $>$ feldspar $>$ pyroxene $>$ ilmenite) has been demonstrated by Pieters and Taylor (2003). Modeling to date of impact melt and vapor production on Mercury relative to the Moon (Cintala 1992) has not included compositional or phase differences such as the lower abundance of plagioclase feldspar or the presence of sulfides (see Sect. 4) within Mercury's regolith (Nittler et al. 2011), so current estimates of melt and vapor production will need to be revised. The lower abundance of feldspar may make current estimates an upper limit, whereas the presence of sulfides (which are more volatile than feldspars) may indicate that current estimates are too low.

Each process we have discussed provides a mechanism for producing the optically important $n \mathrm{pFe}^{0}$, provided there is iron within the regolith. Cosmochemical models for Mercury's formation account for its large iron core but vary in the estimates of crustal iron content (Lewis 1973, 1988; Weidenschilling 1978; Cameron 1985; Fegley and Cameron 1987; Wetherill 1988; Benz et al. 1988, 2007; Ebel and Alexander 2011). The relative rates and efficiencies of operative processes suggest that $\mathrm{npFe}^{0}$ may be more abundant on Mercury than the Moon, depending on the iron abundance in Mercury surface materials. Visible-NIR spectra indicate an absence of ferrous iron in silicates above the 2-wt\% level (e.g., McClintock et al. 2008) and XRS measurements from orbit show an upper limit to the average surface abundance of Fe in any form of $\sim 4 \mathrm{wt} \%$ (Nittler et al. 2011). Comparisons of theoretical reflectance models with MASCS observations suggest that surficial iron has mostly been converted to $\mathrm{nFFe}^{0}$ particles at a variety of sizes (Lucey and Riner 2011). 
The range of mineralogical compositions among asteroids also differs from the surface compositions of the Moon and Mercury. Iron is especially abundant in carbonaceous, ordinary, and enstatite chondrites as metal, sulfides, and $\mathrm{FeO}$ within silicate minerals. Basaltic achondrites, such as those associated with asteroid Vesta, have little macroscopic $(<10 \mu \mathrm{m})$ iron metal and appear less space weathered than main-belt asteroids of chondritic composition. Olivine grains are especially susceptible to space weathering, whereas many opaque minerals (such as ilmenite) are not (Sasaki et al. 2002; Marchi et al. 2005b). However, several studies (Pieters et al. 2000; Hapke 2001; Noble et al. 2007) show that even a modest abundance of $\mathrm{nFFe}^{0}$ on the surfaces of main-belt asteroids is sufficient to produce substantial optical differences from meteorites, as observed. Laboratory analyses of grains returned from asteroid Itokawa demonstrate the presence of other nanophase materials, such as $\mathrm{np}(\mathrm{Fe}, \mathrm{Mg}) \mathrm{S}$, (Noguchi et al. 2011, 2014), which also contribute to the optical effects of space weathering. Studies of Vesta, based on observations returned from the Dawn spacecraft, emphasize the role of gardening and comminution in the maturation of asteroid surfaces, processes that do not require the production of nanophase materials (Pieters et al. 2012; McCord et al. 2012).

\section{Mercury's External Space Environment}

The mercurian and lunar space environments have many similarities, but a major difference between the two bodies is Mercury's internally generated magnetic field. This magnetic field influences solar wind-surface interactions and creates important differences in the weathering effects between Mercury and the Moon.

This section explores Mercury's environment by examining:

- the character of the micrometeoroid population,

- the effects of electric charging of the regolith,

- the character of the solar wind and its population of particles,

- the nature of Mercury's magnetic field and its shielding of the surface, and

- the solar radiation flux, including surface temperature effects.

We also compare Mercury's space environment with that of the lunar and asteroidal environments to evaluate the differences in the rates and efficiencies of environmental processes among different planetary bodies.

\subsection{Micrometeoroid Population}

The primary sources of the micrometeoroid $(<1 \mathrm{~cm})$ population in the inner solar system are asteroids and comets. Understanding of the micrometeoroid population comes from interplanetary dust measurements (including zodiacal dust cloud observations) acquired at Earth orbit (1 AU) and models of the variation of zodiacal light with solar distance (Mann et al. 2004, 2010; Nesvorny et al. 2010). The best estimates of the spatial distribution of interplanetary dust (material 1 to $100 \mu \mathrm{m}$ in size, with a mass range of $10^{-11}$ to $10^{-5} \mathrm{~g}$ ) between 1 and $0.1 \mathrm{AU}$ come from extrapolation of 1-AU measurements. These extrapolations are for dependencies on solar distance $r$ of $r^{-1.0}$ and $r^{-1.5}$ for the number density and flux, respectively (Mann et al. 2004).

Source mechanisms for interplanetary dust within 1 AU of the Sun include PoyntingRobertson deceleration of material originating outward of $1 \mathrm{AU}$, fragmentation by collision between interplanetary grains, and release of material by comets (Mann et al. 2004). Micrometeoroid dust characteristics (such as size, composition, and location) are altered via 
dust collision fragmentation, sublimation of volatile (such as ice) species, radiation pressure acceleration, and sputtering (Mann et al. 2004). Modeling indicates that collisions change the size distribution of interplanetary grains inward of 1 AU (Mann et al. 2004). The composition of interplanetary grains as a function of heliocentric distance is not known. Composition and grain-size distributions are assumed to be similar between Mercury and the Moon for the comparative discussions in this paper.

Studies of nanometer-scale particles (a subset of micrometeoroid dust) indicate that the flux of these particles exceeds that of micrometer-sized dust by at least two orders of magnitude (Kaiser et al. 2007; Czechowski and Mann 2010). The speed of these nano-particles can reach solar wind speeds $(\sim 300 \mathrm{~km} / \mathrm{s})$ at $1 \mathrm{AU}$, so they are a high-energy impact population within the inner solar system (Czechowski and Mann 2010).

\subsection{Electrostatic Charging and Dust Levitation}

Observations and theoretical studies of electrostatically transported dust above the lunar terminator show that submicrometer-sized grains can be levitated and transported tens of kilometers in altitude (e.g., Criswell 1973; McCoy and Criswell 1974; Rennilson and Criswell 1974; Zook and McCoy 1991; Halekas et al. 2002; Stubbs et al. 2006; Colwell et al. 2007; Glenar et al. 2012). The lunar surface is electrostatically charged by the photoemission of electrons due to solar UV, X-rays, and interactions with the local plasma environment (Manka 1973; Halekas et al. 2002). Grains of similar charge to the surface are then levitated by repulsive forces.

Dayside excitation by solar UV and X-rays causes the photoemission of electrons from surface grains, creating a positive potential (Farrell et al. 2007). Dust is also charged by electron and ion impacts, a more complex process (e.g., Abbas et al. 2010). Incident electrons sticking to a dust grain charge it negatively, but the emission of secondary electrons via sputtering produces positive charges. Experimental studies by Abbas et al. (2010) show that charging of dust by electron impact depends on grain size, surface potential, composition, and electron energy and flux. On the lunar nightside, low-density electron plasma current interactions negatively charge the surface (Farrell et al. 2007), thus levitating negatively charged dust grains. The complex electric field at the terminator (where the surface goes from dayside-positive to nightside-negative) transports and mixes the levitated dust (the finer fraction of regolith grains).

No intrinsic magnetic field shields the lunar surface from the plasma environment, though there are localized areas of magnetized crust (e.g., Coleman et al. 1972; Russell et al. 1975; Hood et al. 1981). Lunar swirls (complex, bright albedo patterns not associated with topography) appear only in regions of locally magnetized crust, though not all magnetized regions display swirls (El-Baz 1972; Hood et al. 1979a, 1979b; Hood and Williams 1989; Richmond et al. 2005; Blewett et al. 2010, 2011). From these correlations and measures of soil maturity indices, Blewett et al. $(2010,2011)$ narrowed plausible swirl formation mechanisms to:

- Shielding of the surface from solar wind ion bombardment by a crustal magnetic anomaly, resulting in less space weathering (Hood and Schubert 1980; Hood and Williams 1989)

- Preferential accumulation of fine-grained, feldspar-rich dust by electrostatic levitation induced by solar wind interactions with a local magnetic anomaly (Garrick-Bethell et al. 2011)

If the first process dominates, then solar wind ion bombardment is the main lunar space weathering process (Blewett et al. 2010). If the second dominates, then electrostatic levitation contributes measurably to regolith mixing of the finer soil fraction. Kramer et al. (2011) 
used data from the Moon Mineralogy Mapper instrument on the Chandrayaan-1 spacecraft to determine that swirls have weaker hydroxyl absorptions at $2.82 \mu \mathrm{m}$ wavelength than nonswirl areas. Their finding strongly suggests that the magnetic anomalies are shielding the surface from solar wind ion bombardment and hence maintaining the unweathered spectral characteristics of the swirls. Under both scenarios, then, solar-wind bombardment is likely to dominate over micrometeoroid impact as the main agent of space weathering on the Moon.

The case at Mercury is more complex. Dayside electrostatic levitation of dust grains by photoemission is expected. With the greater solar flux at Mercury than at the Moon, Mercury's surface should be more highly charged, perhaps levitating material to greater altitudes. On the nightside, Mercury's intrinsic magnetic field moderates the creation of plasma electron currents. Though the nightside is generally exposed to electrons from the plasma sheet, MESSENGER's plasma and magnetic field measurements show that reconnection events enhance the exposure of the nightside to electrons in the magnetotail (Slavin et al. 2009, 2010a, 2010b; see also below). These measurements demonstrate, too, that reconnection at the dayside magnetopause and the formation of large flux-transfer events expose the dayside to direct impact of solar wind protons and electrons, especially in the vicinity of the magnetospheric cusps. These dayside reconnection events are also expected to drive highspeed sunward plasma transport in the magnetotail plasma sheet, much of which will impact the nightside surface of Mercury at low latitudes (Slavin et al. 2009, 2010a, 2010b). Flyby images have not revealed albedo markings similar to lunar swirls (Blewett et al. 2010), nor have local regions of highly magnetized crust (i.e., crustal magnetic anomalies) yet been documented (Purucker et al. 2009), although searches continue for both types of features.

Ballistic transport enhanced by electrostatic levitation globally mixes the finer regolith fraction (which dominates optical properties) on the Moon, as exemplified by lunar highland soils (Taylor et al. 2010). Apollo 16 highland agglutinitic glass is enriched in mafic components compared with bulk soil. Taylor et al. (2010) suggested that this enrichment is due to the selective addition of a mare glass component via ballistic transport (impacts accelerate the finer, agglutinitic fraction more and transport it farther) and electrostatic levitation of the finer fraction (Farrell et al. 2008). The differential melting sequence (glass $>$ plagioclase $>$ pyroxene $>$ ilmenite), which includes the preferential melting of mafic-rich (mare composition) glass over Al-rich (highland composition) glass (Taylor et al. 2001a, 2001b), enriches highland agglutinates in mafic material (Taylor et al. 2010). The implications for Mercury are uncertain, but there may be a more globally mixed finer fraction.

The presence of an internally generated magnetic field on Mercury will influence the transport of charged dust to create differences in distribution and interactions with the regolith compared with what is observed on the Moon. The magnetic field (discussed in Sect. 3.4) effects will include removal of charged dust along open field lines in the highlatitude cusp regions and acceleration of charged dust along closed lines at mid and equatorial latitudes. The north-south dichotomy in magnetic field properties (see Sect. 3.4) may create compositional and abundance differences in the fine fraction (which is most easily charged) between the northern and southern high latitudes. Acceleration of the charged dust into the surface may enhance surface grain alterations of the regolith in the mid and equatorial latitudes similar to that produced by micrometeoroid bombardment. These effects have not yet been studied in detail, and the magnitude of their contributions to surface alteration is unquantified.

\subsection{The Interplanetary Particle Environment}

The solar wind, a continuous flow of charged particles (mostly protons and electrons), streams radially from the Sun into the heliosphere (a void in the structure of the interstellar 
Fig. 10 The energy spectrum of thermal and high-energy oxygen nuclei in the heliosphere. The combined data from four instruments on the Advanced Composition Explorer at $1 \mathrm{AU}$ show thermal solar wind as well as solar energetic particle events, co-rotating interaction regions, and cosmic rays. The right axis shows calculated $\mathrm{H}^{+}$at Mercury, using the $\mathrm{H} / \mathrm{O}$ relationships from Table 5. Figure from Mewaldt et al. (2007)

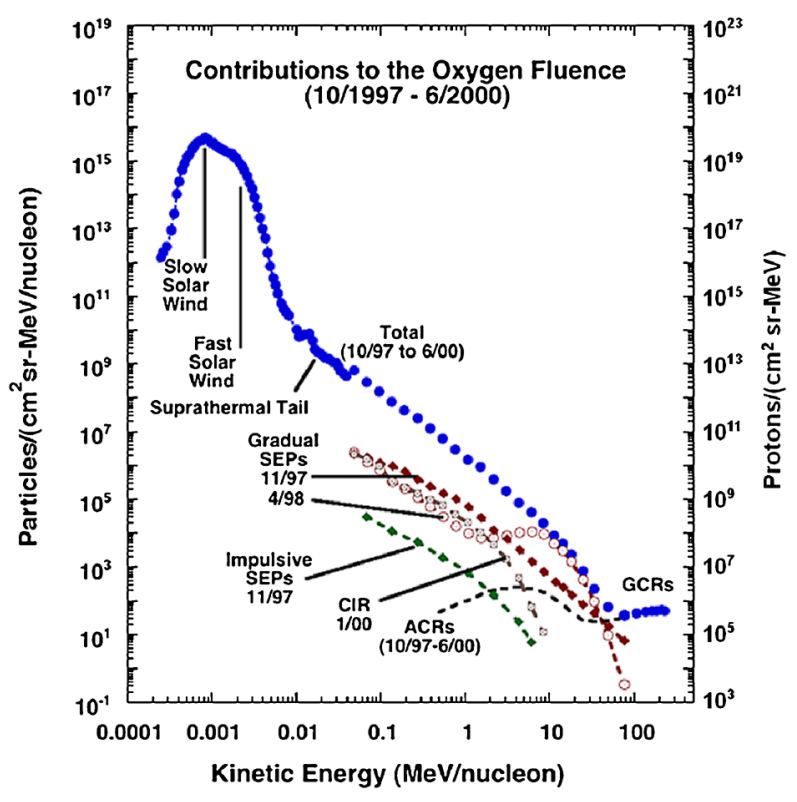

medium created by the solar wind and the frozen-in magnetic field). This section examines solar wind properties and their relation to exosphere generation and surface maturation processes.

\subsubsection{General Properties}

Mercury is surrounded by the solar wind. The space environment at $1 \mathrm{AU}$ is shown in Fig. 10, where O fluxes (left-hand side) are propagated to compute $\mathrm{H}$ fluxes at Mercury's orbit (right-hand side) with simple scaling laws. Solar wind and proton interactions as well as micrometeoroid impacts contribute to Mercury's exospheric density (e.g., Wurz et al. 2010). The relative contributions to surface alteration by various particle radiation processes depend on solar wind dynamic pressure and composition and on the interplanetary magnetic field (IMF) (Wurz and Lammer 2003).

The physical sources of the incident particles may be segregated by energy. Energies ranging up to $5 \mathrm{keV} /$ nucleon are dominated by solar wind; the expanding corona of the slow solar wind nominally consists of a quasi-neutral plasma of $\sim 96 \%$ protons $\left(\mathrm{H}^{+}\right)$and electrons, as well as $\sim 4 \%$ alpha particles $\left(\mathrm{He}^{2+}\right.$ ) and $<0.1 \%$ heavy ions (von Steiger et al. 2000). At the highest energies ( $>100 \mathrm{MeV}$ /nucleon) are galactic cosmic rays (GCRs) that enter the heliosphere from astrophysical sources and interact with the solar wind's turbulent magnetic field. Anomalous cosmic rays (ACRs) are thought to be particles of heliospheric origin accelerated in the heliosphere's boundary regions. They are observable near solar minimum when reduced modulation effects (see below) allow them to re-enter the heliosphere. Particles of energy intermediate to these populations are generally termed suprathermal particles or energetic particles. They originate from transient acceleration in the heliosphere, or from nearly ubiquitous acceleration within the solar wind. This section examines the solar wind particle populations and their time-dependent changes.

Solar Wind Plasma Solar wind plasma escapes from the solar corona and expands supersonically into the heliosphere at speeds of $300-800 \mathrm{~km} / \mathrm{s}$ and at a density of $\sim 10 / \mathrm{r}^{2} \mathrm{~cm}^{-3}$, 
where $r$ is expressed in AU. Solar wind acceleration occurs within 0.1 AU of the Sun. Although both particle density and velocity vary strongly over a solar cycle, in situ measurements of the solar wind bulk properties (e.g., density, velocity, and composition) have traditionally been interpreted in terms of two main types of quasi-stationary wind, "fast" $(600-800 \mathrm{~km} / \mathrm{s})$ and "slow" ( $<450 \mathrm{~km} / \mathrm{s})$. These two solar wind types originate from different solar environments. Heliospheric dynamic processes modify the speed of propagating plasma (e.g., McComas et al. 2002), so recent studies have tended to de-emphasize the fast and slow classifications and instead categorize the solar wind by its charge state composition (Geiss et al. 1995; Gloeckler et al. 2003): solar wind originating in coronal holes (fast) versus solar wind originating in coronal hole boundaries or outside of coronal holes (slow). The first originates in cool solar regions with strong concentrations of open magnetic field lines; the second is likely associated with topologically closed magnetic field structures in the solar atmosphere that release plasma after magnetic interchange reconnection (Gosling et al. 1995; Fisk et al. 1998). The terms "fast" and "slow" are still used, however, for ease of reference.

A third category (Zhao et al. 2009) of heliospheric plasma is transient plasma associated with coronal mass ejections (CMEs). Approximately $15 \%$ of heliospheric plasma during the solar cycle-mostly during times of elevated solar activity-has a transient character. The relative contributions of fast and slow wind depend on the phase of the solar cycle. Slow wind dominates the in-ecliptic plasma near Mercury during solar minimum, but fast wind becomes common during periods of enhanced solar activity near solar maximum

Important compositional characteristics define these three plasma sources. Fast wind has an elemental composition similar to that of the solar photosphere (Grevesse and Sauval 2002). Slow wind and CMEs have compositions enriched in elements with low ( $<10 \mathrm{eV})$ first ionization potentials (FIPs), relative to those with higher FIPs, for example elevating $\mathrm{Fe} / \mathrm{O}$ by a factor of 2-4 over photospheric values (von Steiger and Geiss 1993; Zhao et al. 2009).

The solar wind's ionic charge state reflects its origin. For example, fast solar wind has a substantially lower oxygen charge-state ratio $\left(\mathrm{O}^{7+} / \mathrm{O}^{6+}=0.01\right)$ than slow wind $\left(\mathrm{O}^{7+} / \mathrm{O}^{6+}=\right.$ 0.2 ) or CMEs $\left(\mathrm{O}^{7+} / \mathrm{O}^{6+}>1\right)$ (Zurbuchen et al. 2002; Gloeckler et al. 2003). Solar wind properties at $1 \mathrm{AU}$ are summarized in Table 5, along with some properties near Mercury, as measured by the Helios spacecraft (Marsch et al. 1982; Schwenn 1990). Model fits to the data from MESSENGER's first flyby show that the proton speed, density, and temperature were about $450 \mathrm{~km} / \mathrm{s}, 60 \mathrm{~cm}^{-3}$, and $10^{5} \mathrm{~K}$, respectively (Baker et al. 2009). In the table, $n, v$, and $T$ denote the density, speed, and temperature, and the subscripts for temperature indicate protons $(\mathrm{p})$, electrons (e), and alpha particles $(\alpha)$. The temperature derived from carbon charge states is for conditions of local thermodynamic equilibrium (LTE).

Suprathermal Plasma The plasma in the suprathermal tail of the solar wind distribution is accelerated to energies ranging from $1 \mathrm{keV} /$ nucleon to several $\mathrm{MeV} /$ nucleon, with possible explanations invoking such phenomena as local compressive turbulence (Fisk and Gloeckler 2008; Fisk et al. 2010), acceleration by electric fields from solar wind dynamic processes (Drake et al. 2009), and shock acceleration (Jokipii and Lee 2010).

The suprathermal ion flux expected at Mercury during times of slow solar wind flow is over $10^{5} \mathrm{~cm}^{-2} \mathrm{~s}^{-1}$, whereas the solar wind flux is $\sim 3,000$ times greater; suprathermal ions are nonetheless important at Mercury's surface. Depending on IMF conditions, portions of the surface are shielded from the solar wind by Mercury's internal magnetic field (Kabin et al. 2000; Kallio and Janhunen 2003; Sarantos et al. 2007; Benna et al. 2010). The shielding is substantially less efficient for suprathermal particles, however, which are higher in energy and nearly omnidirectional. These ions can directly impact and weather the surface during times when the bulk solar wind is deflected by the magnetic field. 
Table 5 Mean solar wind properties at Earth and Mercury distances from the Sun

\begin{tabular}{|c|c|c|c|c|}
\hline \multirow[t]{2}{*}{ Parameter } & \multicolumn{2}{|c|}{ Slow wind } & \multicolumn{2}{|c|}{ Fast wind } \\
\hline & $1 \mathrm{AU}$ & $0.4 \mathrm{AU}$ & $1 \mathrm{AU}$ & $0.4 \mathrm{AU}$ \\
\hline$n\left(\right.$ protons $\left./ \mathrm{cm}^{-3}\right)$ & 8.3 & 74.1 & 2.7 & 20.8 \\
\hline$v(\mathrm{~km} / \mathrm{s})$ & 327 & 348 & 702 & 667 \\
\hline$T_{\mathrm{p}}\left(\times 10^{5} \mathrm{~K}\right)$ & 0.34 & 0.55 & 2.3 & 2.8 \\
\hline$T_{\mathrm{e}}\left(\times 10^{5} \mathrm{~K}\right)$ & 1.3 & 1.9 & 1.0 & 1.3 \\
\hline$T_{\alpha}\left(\times 10^{5} \mathrm{~K}\right)$ & 1.1 & 1.7 & 1.4 & 7.3 \\
\hline $\begin{array}{l}\text { LTE carbon charge-state } \\
\text { temperature }(\mathrm{K})\end{array}$ & \multicolumn{2}{|c|}{$8 \times 10^{5}$} & \multicolumn{2}{|c|}{$(1.4-1.6) \times 10^{6}$} \\
\hline $\mathrm{O}^{7+} / \mathrm{O}^{6+}$ & \multicolumn{2}{|l|}{0.2} & \multicolumn{2}{|l|}{0.01} \\
\hline $\begin{array}{l}\text { Average Fe charge state } \\
\text { and temperature }(\mathrm{K})\end{array}$ & \multicolumn{2}{|c|}{$11.2\left(1.4 \times 10^{6}\right)$} & \multicolumn{2}{|c|}{$10.1\left(1.2 \times 10^{6}\right)$} \\
\hline $\mathrm{He} / \mathrm{H}$ & \multicolumn{2}{|l|}{0.01} & \multicolumn{2}{|l|}{0.048} \\
\hline $\mathrm{H} / \mathrm{O}$ & \multicolumn{2}{|l|}{2300} & \multicolumn{2}{|l|}{1500} \\
\hline $\mathrm{C} / \mathrm{O}$ & \multicolumn{2}{|l|}{0.67} & \multicolumn{2}{|l|}{0.68} \\
\hline $\mathrm{Fe} / \mathrm{O}$ & \multicolumn{2}{|l|}{0.12} & \multicolumn{2}{|l|}{0.09} \\
\hline $\mathrm{Mg} / \mathrm{O}$ & \multicolumn{2}{|l|}{0.15} & \multicolumn{2}{|l|}{0.11} \\
\hline
\end{tabular}

Properties measured by Helios 1 and 2 and IMP $7 / 8$ (Schwenn 1990). Ionic charge-state ratios and temperatures at $1 \mathrm{AU}$ are from Ipavich et al. (1998), Lepri et al. (2001), and Zurbuchen et al. (2002); elemental abundances at $1 \mathrm{AU}$ are from Bame et al. (1977), Bochsler (1998), and von Steiger et al. (2000, 2010). A more complete list can be obtained from these sources

High-Energy Particles Heliospheric plasma with energies above that of the solar wind include high-energy particles ejected from the Sun, such as solar energetic particles (SEPs), and particles that gain energy by acceleration in the heliosphere. High-energy particles propagate almost freely within the heliosphere, making particle fluxes near Mercury highly transient and nearly unpredictable.

SEP events contain plasma with MeV/nucleon energies and are characterized as impulsive (on timescales of minutes to hours) or gradual (hours to days), though such a distinction is somewhat arbitrary (Klecker et al. 2006). Impulsive events are flare-associated, containing particles with high charge states indicating temperatures in the range $(2-7) \times 10^{6} \mathrm{~K}$ (Luhn et al. 1984). From $\sim 10$ to $50 \mathrm{MeV} /$ nucleon, composition data reveal that Fe-rich impulsive events are primarily accelerated solar flare plasma (Mewaldt et al. 2007). Gradual events, associated with expanding coronal mass ejections, contain plasma characteristic of fast CMEs and possibly suprathermal particles near the Sun (Reames et al. 1999; Desai et al. 2006). Their elemental composition does not match the bulk solar wind (von Steiger et al. 2000; Mewaldt et al. 2007). Preceding the higher-speed CMEs is a component consisting of shocked solar wind.

Particles in the heliosphere can be accelerated by shocks, such as those forming at the edges of co-rotating interaction regions (CIRs). These shocks contain pickup ions and fast solar wind (Gloeckler et al. 1994). Such CIRs typically form at heliospheric distances of $>1 \mathrm{AU}$ and are not expected to be an important factor near Mercury. Observations from the Helios spacecraft have shown that energetic particle events in the inner solar system (sunward of the asteroid belt) are highly structured and transient, although they appear merged at larger ( $>4 \mathrm{AU})$ heliospheric distances. 
Cosmic Rays and Anomalous Cosmic Rays ACRs are thought to be pickup ions that traveled beyond the termination shock of the solar wind, experienced acceleration, and returned to the heliosphere. The composition of ACRs matches both interstellar and innersource pickup ions (Gloeckler and Geiss 2001), and their energies range from $\sim 10$ to $50 \mathrm{MeV} /$ nucleon.

Galactic cosmic rays (GCRs) are mostly protons (86\%), alpha particles (8\%), and electrons (5\%), with trace amounts of heavy ions (Evenson et al. 1983; Mewaldt 1994). They are detected at $1 \mathrm{AU}$ with energies $>100 \mathrm{MeV}$ /nucleon.

\subsubsection{Observations of the Mercury Plasma Environment}

Data collected by MESSENGER's Fast Imaging Plasma Spectrometer (FIPS) and Magnetometer (MAG) have revealed that certain regions of Mercury's magnetosphere have high fluxes of heavy ions (i.e., heavier than protons). The measured fluxes of ions with mass per charge near that of singly ionized sodium and oxygen increase near the magnetic cusp of Mercury's northern hemisphere, near the periapsis of MESSENGER's orbit. This enhancement is likely due to greater ion sputtering at high latitudes as a result of lower shielding of the surface by the magnetic field. Helium ions, which are also seen in relatively large quantities, have a more uniform distribution in the magnetosphere, consistent with a more spatially uniform source such as solar-wind-implanted helium evaporated from Mercury's surface (Zurbuchen et al. 2011).

\subsubsection{Solar Wind and Interplanetary Magnetic Field near Mercury}

The IMF carried by the solar wind affects the interaction of solar wind particles with Mercury's magnetic field. Probability densities of solar wind conditions at Mercury computed from Helios data inside Mercury's orbit (Fig. 11) provide insight into Mercury's local space environment (Sarantos et al. 2007). (In this figure and elsewhere in this paper we adopt the Mercury solar orbital coordinate system, in which $X$ is positive sunward, $Y$ is positive in the direction opposite to Mercury's orbital motion, and $Z$ is positive northward, i.e., normal to Mercury's orbital plane and toward the north celestial pole.) Evident in Fig. 11 is the seasonal variation of the driving forces at Mercury: an average IMF strength of $\sim 40 \mathrm{nT}$ and a solar wind pressure up to tens of nPa near Mercury's perihelion. Given Mercury's location in the inner heliosphere, the planet's magnetosphere is subjected to low-Mach-number (highly subsonic) flows. This flow environment enhances the magnetic reconnection efficiency between the planetary and interplanetary magnetic fields (Slavin and Holzer 1979), resulting in increased access of solar wind plasma to Mercury's surface. We next describe the current understanding of Mercury's magnetic field, its interactions with the solar wind environment, and its effects on solar wind-surface interactions.

\subsection{Planetary Magnetic Field}

A global planetary magnetic field can greatly impede access of solar wind ions to a planetary surface, thereby reducing rates of space weathering by sputtering of regolith-derived exospheric species or solar-wind-induced chemical processes in the soil. The effectiveness of magnetic shielding depends on solar wind conditions and the strength of the planetary and interplanetary magnetic fields. Mercury's surface is susceptible to solar wind impact because of its lack of a dense atmosphere, the weak internal field, and the strong solar wind and IMF environment, but to a somewhat lesser extent than that of the Moon or asteroids. In comparisons with the Moon and asteroids, it is important to understand how Mercury's magnetic field affects rates of particle irradiation processes. 

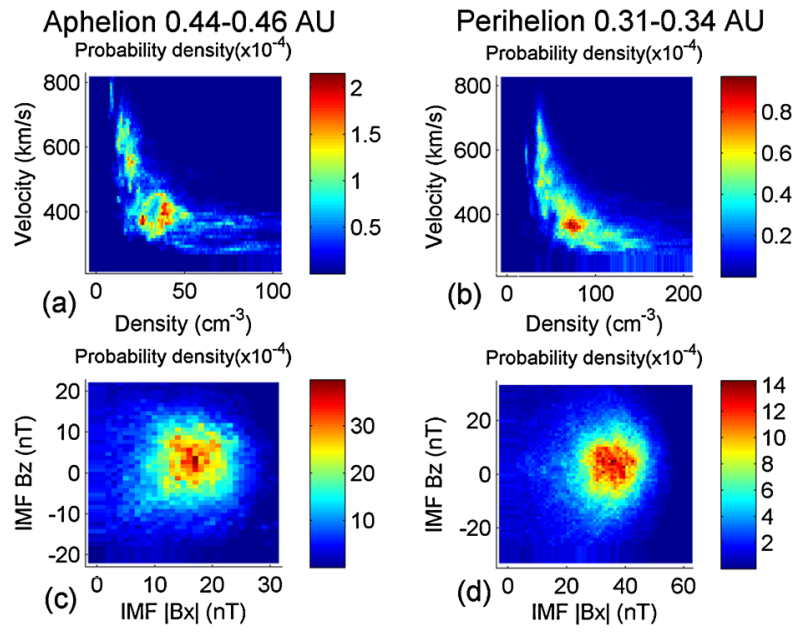

Fig. 11 Probability density of solar wind conditions at Mercury derived from data from the Helios spacecraft. (a, b) Distributions of solar wind density versus solar wind velocity. (c, d) Distribution of the magnitudes of the northward and sunward components of the interplanetary magnetic field $\mathbf{B}, B_{x}$ and $B_{z}$, respectively. These distributions were computed (a, c) near Mercury aphelion (0.44-0.46 AU) and (b, d) near perihelion (0.31-0.34 AU). The bin sizes in these plots are as follows: density, $1 \mathrm{~cm}^{-3}$; velocity, $10 \mathrm{~km} / \mathrm{s}$; and IMF components, 1 nT. Figure from Sarantos et al. (2007)

\subsubsection{Mercury's Field and Its Dynamic Magnetosphere}

Knowledge of Mercury's magnetic field, discovered during the Mariner 10 flybys in 19741975, has been greatly extended by the three MESSENGER flybys in 2008-2009 (Anderson et al. 2008, 2010; Slavin et al. 2008, 2009, 2010a, 2010b) and by Mercury orbital observations (Anderson et al. 2011). Observations from the two spacecraft indicate that Mercury's planetary field is stable over long timescales. MESSENGER flyby measurements confirmed the inference from Mariner 10 observations that the magnetic field is primarily dipolar and indicated a dipole moment of about 190nT- $R_{\mathrm{M}}^{3}$, where $R_{\mathrm{M}}$ is Mercury's radius (Johnson et al. 2012). MESSENGER orbital measurements demonstrated that Mercury's magnetic dipole is displaced northward of the geographic equator by a mean distance of $484 \pm 11 \mathrm{~km}$; the internal dipole of moment $195 \mathrm{nT}-R_{\mathrm{M}}^{3}$ is aligned with the spin axis to within $3^{\circ}$ (Anderson et al. 2011).

The northward displacement of Mercury's dipole field creates a difference in the properties of the surface field and magnetospheric cusp area between the north and south polar regions. The surface field at the north pole is 3.4 times larger than at the south pole, whereas the area of the surface at which lines of magnetic flux open to space outside the magnetosphere is four times larger in the southern hemisphere than in the northern hemisphere (Anderson et al. 2011), creating hemispherical differences in solar wind access to the surface. From six months of MESSENGER MAG observations, Winslow et al. (2012) showed that the northern cusp region is always present but its extent and the depth of its magnetic depression vary strongly with solar wind conditions. Under anti-sunward IMF, the magnetic depression is $20 \%$ stronger and plasma pressure is $40 \%$ higher in the northern cusp region than when the IMF is oriented sunward (Winslow et al. 2012). From their analysis, Winslow et al. (2012) determined that the mean area of the northern cusp area at Mercury's surface is $(5.2 \pm 1.6) \times 10^{11} \mathrm{~m}^{2}$, whereas the corresponding area for the southern cusp is predicted to 
Fig. 12 Simulation of the effect of $1100 \mathrm{~km} / \mathrm{s}$ solar wind compressing Mercury's subsolar magnetopause to a point below the planet's surface and exposing the regolith at that point to direct bombardment by the solar wind. Color scale shows particle density. Figure from Kabin et al. (2000)

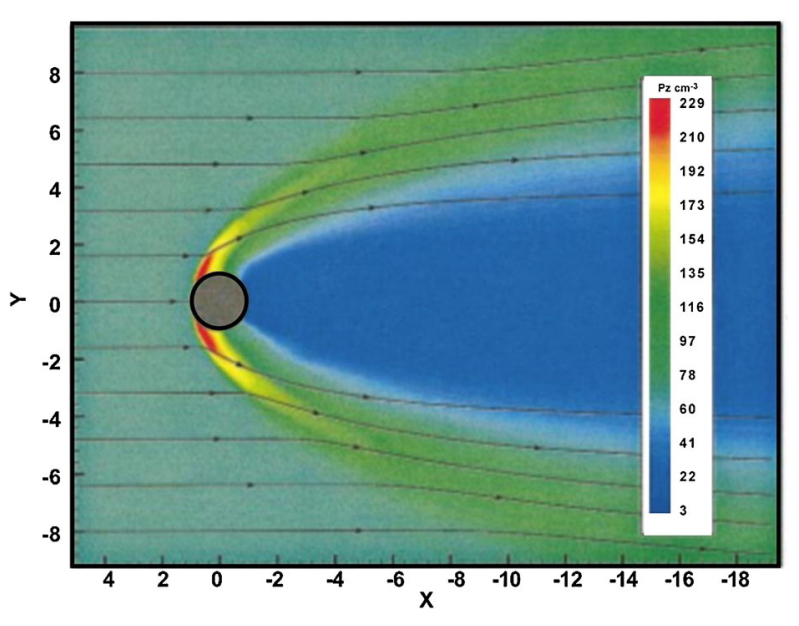

be four times higher $\left(2 \times 10^{12} \mathrm{~m}^{2}\right)$. The central latitude of the northern cusp area is $\sim 74^{\circ} \mathrm{N}$, with an average latitudinal extent of $19^{\circ}$ (Winslow et al. 2012). It is important to realize, however, that this extent is an average and that the cusp has been observed to extend in extreme limits from 55.8 to $83.6^{\circ} \mathrm{N}$, a latitudinal extent of $\sim 28^{\circ}$ (Winslow et al. 2012). The central latitude of the southern cusp is calculated to be $\sim 64^{\circ} \mathrm{S}$, and the cusp region is expected to extend $\sim 38^{\circ}$ in latitude on average (Winslow et al. 2012). Winslow et al. (2012) estimated that on average $(1.1 \pm 0.6) \times 10^{24}$ protons per second reach Mercury's surface in the northern cusp region, and the flux is a factor of four higher in the southern cusp. These north-south asymmetries could produce hemispherical differences in exosphere generation and space weathering (Winslow et al. 2012).

In addition, Mercury's weak internal field means that the average distance to the subsolar point on the magnetopause is $1.45 R_{\mathrm{M}}$ from planetary center and varies with solar wind forcing (Fig. 11, Sarantos et al. 2007; Winslow et al. 2013) At times of sufficiently high solar wind ram pressure (e.g., interplanetary shocks and CMEs), the magnetopause may be compressed to the surface (e.g., Kabin et al. 2000; Kallio and Janhunen 2003) and, if so, the dayside can become open to the solar wind (Benna et al. 2010) as shown in Fig. 12.

Another mechanism for reducing the magnetospheric obstacle is erosion of dayside magnetic flux by reconnection. MESSENGER flyby measurements demonstrated that Mercury's magnetosphere is extremely variable on timescales of minutes as a result of reconnection of the IMF with the planetary magnetic field (Slavin et al. 2009, 2010a, 2010b). Two forms of variability are (1) the recurrent presence and spatially extended nature of magnetic fluxtransfer events (FTEs) under southward IMF at Mercury (Slavin et al. 2008, 2010a) and (2) the recurrent presence of multiple plasmoids, traveling compression regions, and cycles of loading and unloading in Mercury's magnetic tail region (Slavin et al. 2010b). The repeated appearance of these events suggests that a succession of global compressions of the forward magnetosphere and episodes of reconnection in the tail can be produced by variations in the direction of the IMF component normal to Mercury's orbital plane (the north-south component). MESSENGER MAG observations acquired from orbit have shown the sign of this IMF component to be highly variable (Slavin et al. 2012). These observations indicate that Mercury's magnetosphere is much more responsive to IMF direction and more dominated by the effects of reconnection than those of Earth or other planets that host internal magnetic fields (Slavin et al. 2009, 2010b). 
The consequence of these observed reconnection effects is that Mercury's magnetosphere is much more permeable to the solar wind than that of Earth. FTEs are episodes of reconnection of the planetary field with the IMF on the dayside magnetopause, during which magnetic flux is transferred from the dayside to the magnetic tail. An average measured FTE diameter at Mercury is $\sim 900 \mathrm{~km}\left(\sim 0.4 R_{\mathrm{M}}\right)$, or $28 \%$ of the $1.4 R_{\mathrm{M}}$ mean distance from the center of the planet to the subsolar nose of the magnetopause (Slavin et al. 2009, 2010a). Such large FTEs markedly disturb the topology of the entire dayside magnetosphere, and thus the flux of solar wind ions reaching the surface. The largest FTE observed during the MESSENGER flybys had an inferred diameter of $1 R_{\mathrm{M}}$, and its open magnetic field increased the fraction of the surface exposed to the solar wind by $\sim 10-20$ percent over typical IMF conditions (Slavin et al. 2010a). The actual increase in precipitating flux could be much greater, given that these perturbations connect the mid-latitude surface to plasma from the densest, most compressed region near the nose of the magnetopause, which was previously thought to be excluded from access to the surface (e.g., Sarantos et al. 2007) except during the most disturbed solar wind events (Kallio and Janhunen 2003). Even greater solar wind impact flux, especially at low latitudes on the dayside hemisphere, may occur during reconnectiondriven cycles of magnetic flux "loading" and "unloading" of the magnetic tail, as observed by MESSENGER during its third flyby, when the IMF north-south component was variable (Slavin et al. 2010b). The transfer of magnetic flux into the tail reduces the effective magnetic field obstacle that the solar wind encounters on the dayside, therefore increasing accessibility. The tail flux at the time of the peak loading events was estimated to be at least $30 \%$, and for the most intense event possibly $100 \%$, of the available magnetic flux from Mercury's dayside (Slavin et al. 2010b). Under such extreme conditions, the entire magnetic flux content of the dayside may be pulled back into the tail, exposing the equatorial surface to the solar wind for $\sim 1$ min at a time, and up to $100 \%$ of the shocked solar wind may access the surface. Such an event is followed, during the unloading phase, by intense precipitation of plasma onto Mercury's nightside minutes later. Just how intense the supply of solar wind ions to the surface may be during active magnetospheric conditions is a topic that warrants further study.

\subsubsection{Interactions of the Surface with the IMF and Solar Wind}

Models of solar wind-planet interactions, which help elucidate the degree of surface weathering, predict that large portions of Mercury's surface are exposed to the solar wind even when magnetospheric reconnection is absent. With such an open magnetosphere, access to the surface by solar wind ions may be only slightly lower at Mercury than at the Moon.

The steady-state rate of access of solar wind ions to Mercury's mid- and high-latitude surface through the outer boundary of the magnetosphere (i.e., the magnetopause, and the two magnetospheric cusps) was estimated by Sarantos et al. (2007). In that study, 40-min averaged data from the Helios spacecraft were used to describe Mercury's solar wind environment, and those data were combined with a model of Mercury's magnetosphere to evaluate the solar wind precipitation rate. It was found that this rate varies from $(2-4) \times 10^{25}$ ions s $^{-1}$ at aphelion to $(8-15) \times 10^{25}$ ions s $^{-1}$ at perihelion in Mercury's eccentric orbit. These estimates, obtained with the assumption of a geographically centered Mercury dipole moment of $350 \mathrm{nT}-R_{\mathrm{M}}^{3}$, are supported by those from a number of other magnetospheric models (e.g., Kallio and Janhunen 2003; Trávníček et al. 2007, 2009, 2010; Massetti et al. 2007; Benna et al. 2010). However, as noted above, the northward displacement of Mercury's dipole (Anderson et al. 2011) creates a hemispherical difference in ion flux to the surface in the cusp regions. 
Fig. 13 Maps of the flux of protons precipitating onto the surface of Mercury during MESSENGER's first (M1, top) and second (M2, bottom) flybys modeled from Magnetometer measurements (Benna et al. 2010). Longitudes correspond to local time of day, where $0^{\circ}$ longitude corresponds to local noon. The fluxes are normalized to the flux of the undisturbed solar wind

$\left(9 \times 10^{8}\right.$ particles $/ \mathrm{cm}^{2} / \mathrm{s}$ during M1 and $6 \times 10^{8}$ particles $/ \mathrm{cm}^{2} / \mathrm{s}$ during M2)
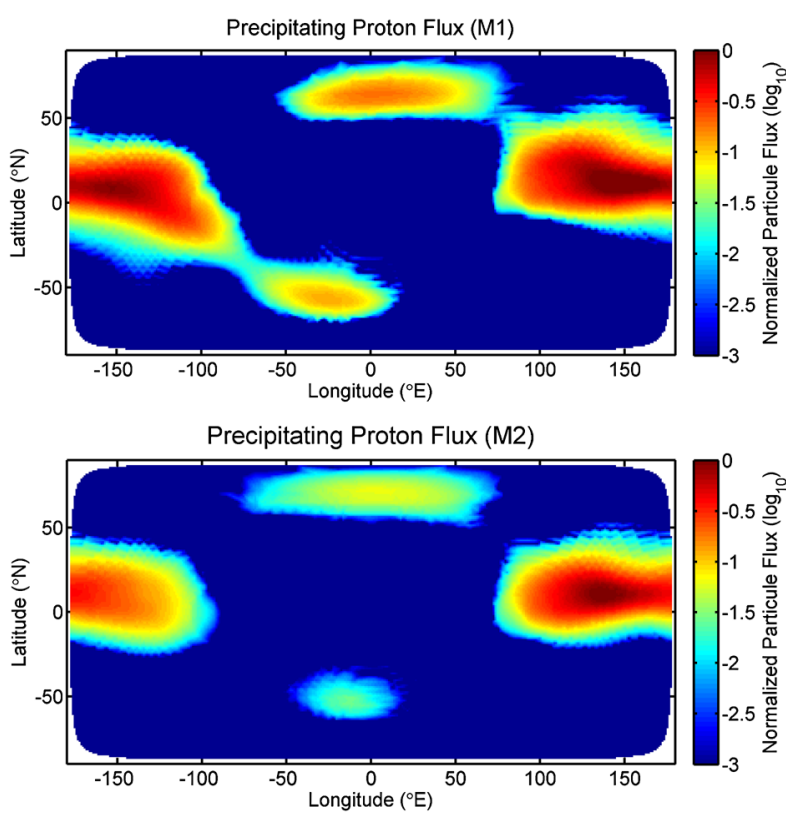

The region accessible to the solar wind is regulated mainly by IMF orientation and the dynamic pressure of the solar wind, but the entry of solar wind and the related weathering effects are not confined to high-latitude regions. Large portions of the near-equatorial nightside are exposed to plasma-sheet plasma flowing sunward from the more distant magnetotail. The high reconnection rate at Mercury places the magnetic separatrix between the planetary field and IMF very near the planet's nightside, $\sim 1-3 R_{\mathrm{M}}$ above the surface according to MESSENGER data (Slavin et al. 2010a, 2010b). Under this intense but steady-state forcing, magnetospheric models suggest that large portions of the nightside are regularly bombarded by the energetic ions and electrons from the plasma sheet (Fig. 13). Plasma impact on the nightside of Mercury is expected to be greatly enhanced under active magnetospheric conditions. The MESSENGER XRS measured several count-rate spikes near closest approach during all three Mercury flyby events attributed to $\sim 1-10 \mathrm{keV}$ electrons interacting with the XRS detector material (Ho et al. 2011a). During orbital operations the XRS has detected energetic electrons on nearly every orbit (Ho et al. 2011b) and electron-induced X-ray florescence from Mercury's nighttime surface, confirming the precipitation of these electrons to the surface (Starr et al. 2012).

A simulation of electron transport from the solar wind through Mercury's magnetosphere for solar wind conditions similar to those during the first two MESSENGER flybys of Mercury showed that precipitating electron fluxes were as large as $10^{9}-10^{10} \mathrm{~cm}^{-2} \mathrm{~s}^{-1}$ in some places, with average energies from hundreds to $\sim 1000 \mathrm{eV}$, as illustrated in Fig. 14 (Schriver et al. 2011a). Precipitating electron energies were generally lower during the first MESSENGER flyby (M1) when the IMF had a northward component, and were on average higher during the second flyby (M2), when the IMF had a southward component (during both encounters the IMF had a large radial component). Simulations matched to orbital observations have provided insight into the dependence of ion and electron precipitation flux on solar wind IMF properties.

An example of the excellent correlation between MAG and FIPS orbital observations and ion density contour maps derived from global hybrid simulations is shown in Fig. 15 
Fig. 14 (Top) Electron precipitation flux and (bottom) electron precipitation energy for a simulation with solar wind parameters corresponding to MESSENGER's first flyby of Mercury (Schriver et al. 2011a). Both panels show electron data as a function of latitude versus longitude, with $0^{\circ}$ latitude corresponding to the equator, $90^{\circ}$ latitude the north pole, $-90^{\circ}$ latitude the south pole. Longitudes are equivalent to local time of day, with $0^{\circ} / 360^{\circ}$ longitude corresponding to midnight, $90^{\circ}$ longitude dawn, $180^{\circ}$ longitude noon, and $270^{\circ}$ longitude dusk
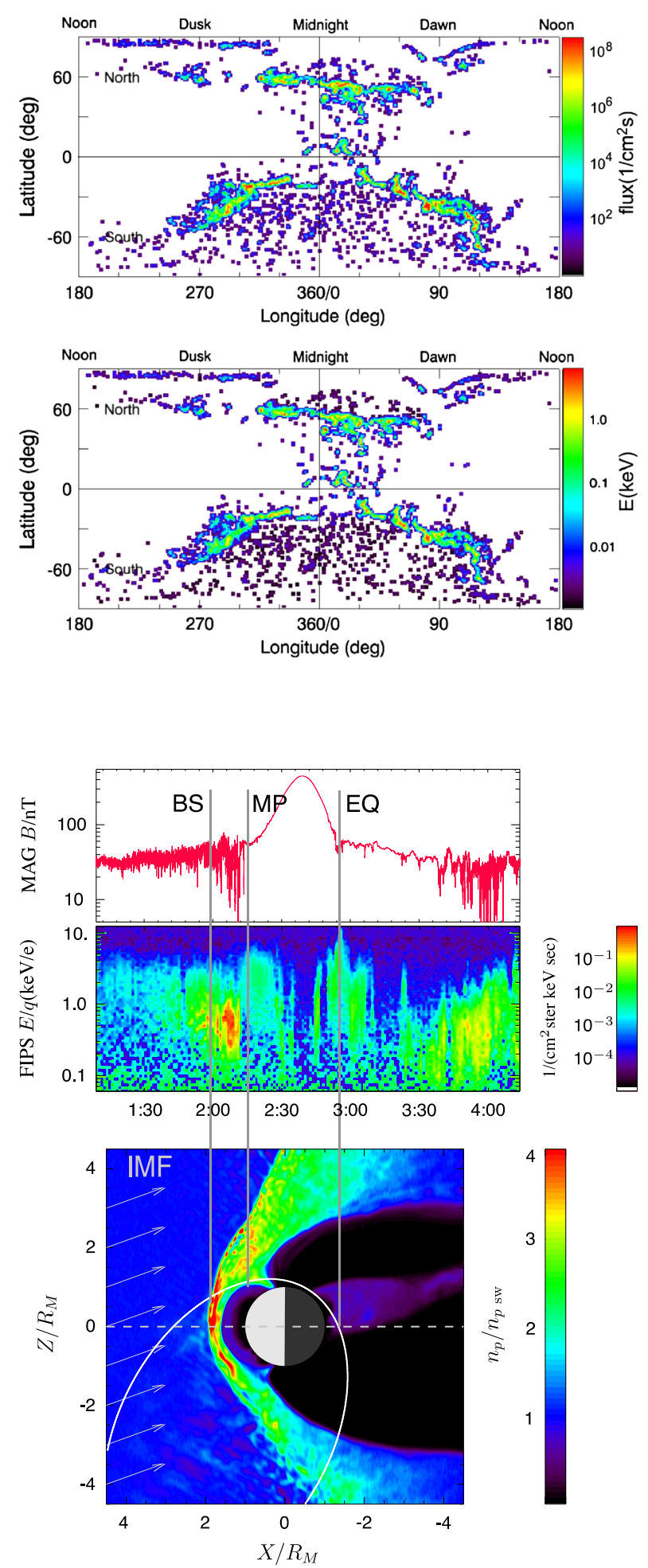

Fig. 15 Comparison of MESSENGER orbital measurements of magnetic field and plasma ion flux with global hybrid simulations of the distribution of plasma ions near Mercury. The top two panels show $\sim 3$ hours of data from MAG (total magnetic field in $\mathrm{nT}$ ) and FIPS (ion energy spectrogram, in $\mathrm{keV}$, color coded by energy flux) on 31 March 2011, respectively, and the bottom panel displays contours of ion density (normalized to solar wind density) from a global hybrid simulation in the noon-midnight meridian $(Z-X$ plane, $Y=0)$. The

MESSENGER orbit is shown in white on the bottom panel, and the arrows at left show the orientation of the IMF. The bow shock $(B S)$, magnetopause $(M P)$, and nightside geographic equator $(E Q)$ locations are indicated (Schriver et al. 2011b) 
Fig. 16 An example of the simulated plasma proton density $n_{\mathrm{p}} / n_{\mathrm{psw}}$, where $n_{\mathrm{psw}}$ is the corresponding density in the solar wind, from a global hybrid simulation (the simulation denoted g4 in Table 6, with the IMF pointing northward and anti-sunward) (Schriver et al. 2011b). The upper panel shows the simulated proton density in the equatorial $(X-Y)$ plane, and the bottom panel shows the simulated proton density in the plane $(X-Z)$ of the noon-midnight meridian. The grey circle denotes Mercury in both panels
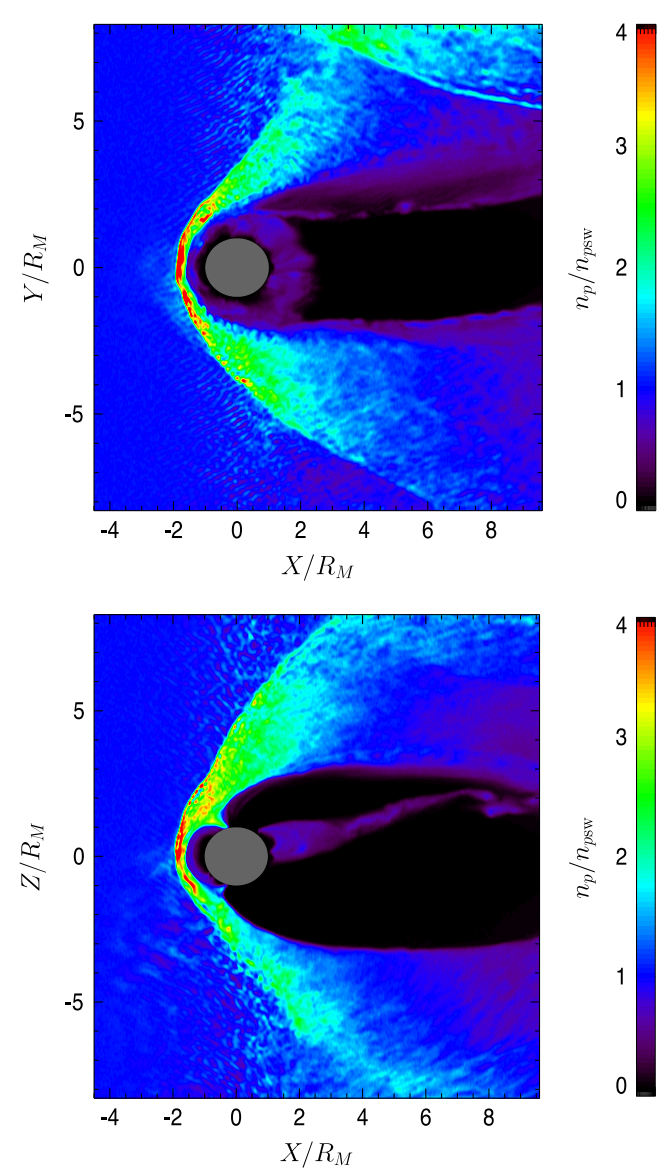

(Schriver et al. 2011b). Additional details of this simulation are shown in Fig. 16. These global hybrid simulations serve as inputs to derivations of the ion precipitation flux onto Mercury's surface. This flux varies with both the $X$ direction (sunward versus anti-sunward) and $Z$ orientation (north, south, or equatorial) of the IMF, as demonstrated in Fig. 17. A summary of the IMF conditions in the simulations corresponding to the precipitation maps in Fig. 17 is provided in Table 6.

The simulations with anti-sunward IMF orientations all show higher precipitation in the southern than the northern hemisphere, and lower flux when the IMF is oriented equatorward. When the IMF is oriented northward there appears to be an enhanced flux on the dusk side compared with the dawn side. However during southward-oriented IMF conditions the opposite appears to occur, and there is an enhanced flux on the dawn side compared with the dusk side. The models with sunward IMF orientations all show a stronger nightside, northern-hemisphere enhancement that is not apparent under anti-sunward orientations. In the sunward IMF cases, both the southward and equatorward orientations show a dawn-side enhancement in electron precipitation relative to the dusk side. The northward orientation, however, shows no discernable enhancement at either the dawn or dusk regions. These results can be used to predict surface depletion of volatile species within Mercury's regolith as a function of latitude. 

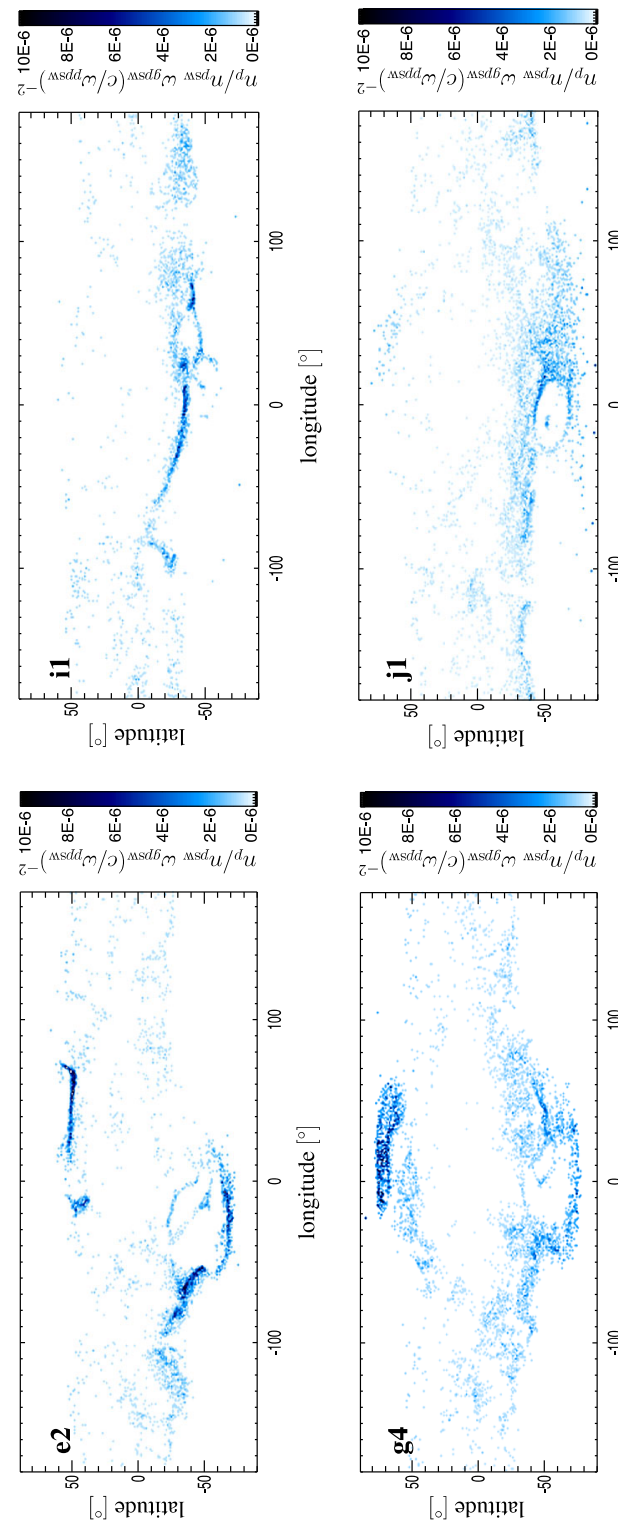
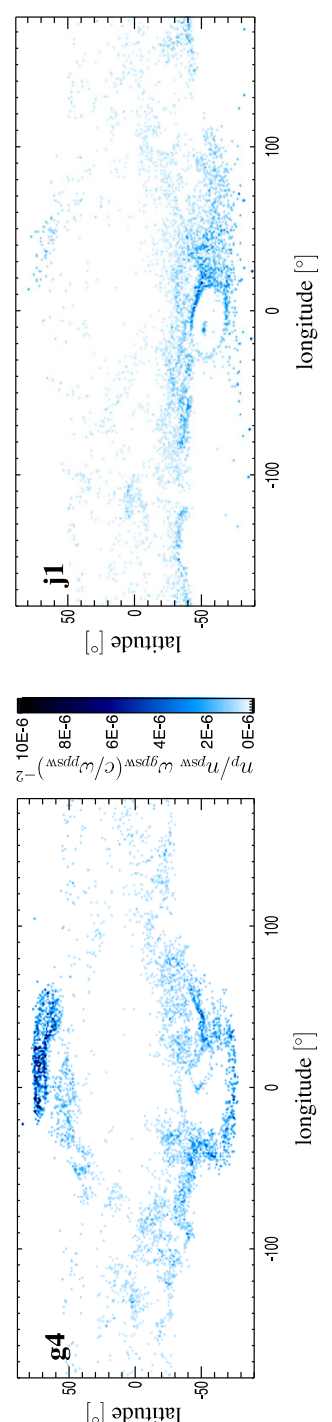
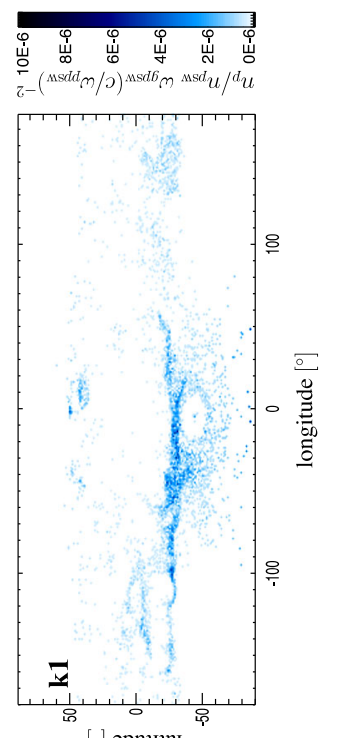

[o] әрпц!̣ए I

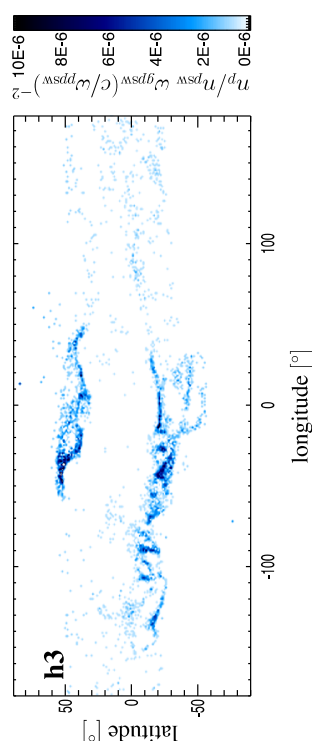

马्ष

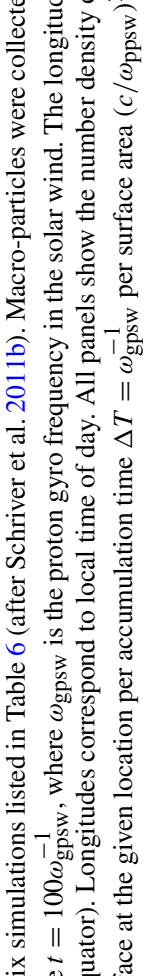

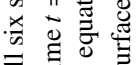

न $\Xi$ 向 क

$\Xi \tilde{\Xi} \approx$

ग्ण

完离

뉴

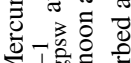

和造

ㅇ.

证文

考宁券

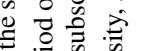

율 के क्ष

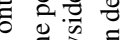

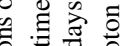

\%

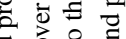

욜

帘 :

क्षे

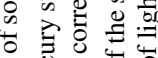

ธี

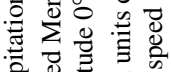

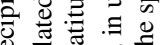

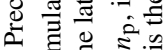

D.

ح क

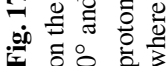


Table 6 IMF conditions for the global hybrid simulations of plasma ion distribution near Mercury shown in Fig. 17

\begin{tabular}{|c|c|c|c|}
\hline IMF & Northward & Southward & In equatorial plane \\
\hline Sunward & $\begin{array}{l}\text { Simulation } \mathrm{j} 1 \text { : } \\
\left(B_{X}<0, B_{Y}=0, B_{Z}>0\right)\end{array}$ & $\begin{array}{l}\text { Simulation k1: } \\
\left(B_{X}<0, B_{Y}=0, B_{Z}<0\right)\end{array}$ & $\begin{array}{l}\text { Simulation i1: } \\
\left(B_{X}<0, B_{Y}>0, B_{Z}=0\right)\end{array}$ \\
\hline Anti-sunward & $\begin{array}{l}\text { Simulation g4: } \\
\left(B_{X}<0, B_{Y}=0, B_{Z}>0\right)\end{array}$ & $\begin{array}{l}\text { Simulation h3: } \\
\left(B_{X}>0, B_{Y}=0, B_{Z}<0\right)\end{array}$ & $\begin{array}{l}\text { Simulation e2: } \\
\left(B_{X}>0, B_{Y}<0, B_{Z}=0\right)\end{array}$ \\
\hline
\end{tabular}

Note: These six global hybrid simulations of solar wind interaction with Mercury's magnetosphere are for six different orientations of the IMF B. All other parameters in the simulations were identical. The solar wind speed was taken to be 4 times larger than the Alfvén speed in the solar wind. The angle of the IMF within the $X-Z$ plane with respect to the equatorial plane (when nonzero) was set to $20^{\circ}$; the angle of the IMF with respect to the solar wind flow within the equatorial $(X-Y)$ plane (when nonzero) was set to $45^{\circ}$

The timescale for circulation of plasma, magnetic flux, and energy from the dayside magnetosphere to the magnetotail and subsequently back to the dayside magnetosphere (the socalled Dungey cycle) is very short for Mercury, $\sim 1-2 \mathrm{~min}$. These times are short compared with diffusive or gardening timescales, so even brief changes effected in the magnetosphere by reconnection have an opportunity to alter the surface over long timescales.

The energetic particle population at Mercury was first reported on the basis of observations made during the first Mercury flyby of Mariner 10 in 1974 (Simpson et al. 1974). From orbital observations, MESSENGER has shown that there are regular bursts of energetic electrons $(<300 \mathrm{keV}$ ) inside Mercury's magnetosphere, but no evidence has been seen of high-energy protons as reported from Mariner 10 observations (Ho et al. 2011a, 2011b). These electrons typically have energies of $100 \mathrm{keV}$ or less and are detected mostly on the nightside at high latitude. The location and angular distribution of these energetic electrons suggest that there are no fully trapped energetic particles at Mercury. These electrons are sufficiently energetic that they can follow Mercury's magnetic field lines and directly impact the surface. The recurrent nature of these electron events means that whatever acceleration mechanism is responsible operates on a nearly continuous basis. Electron-induced X-ray fluorescence from this electron population has been detected by the MESSENGER XRS and used to determine elemental abundances of $\mathrm{Mg}, \mathrm{Al}, \mathrm{Si}, \mathrm{S}$, and $\mathrm{Ca}$ from localized regions on the surface (Starr et al. 2012).

\subsection{The Sun through Time}

Variations in the Sun's photon and solar wind output affect each space weathering process discussed above, through variations in temperature, particle flux and composition, and photon flux. The properties of the dynamic solar wind change on timescales ranging from hours to many millennia. Because solar wind evolution is directly tied to dynamic changes in the Sun, solar wind measurements can reveal internal solar processes. By examining solar variability through the sunspot record or proxies such as terrestrial ice cores, solar wind traits can be inferred and correlated with planetary surface processing over time. In this section we examine how the solar output has varied over these timescales and assess the implications for the effectiveness of space weathering processes on Mercury's surface.

\subsubsection{Short-Timescale Variations}

Transient Evolution Short-timescale variability, on the order of hours, originates from the scale length of coronal structures and the plasma's evolution as it propagates through the 
inner heliosphere. Slower and faster streams may interact, leading to compressive fluctuations or rarefaction regions. CMEs (including SEPs) are probably the dominant perturbations near Mercury. They can be hurled into space at $2000 \mathrm{~km} / \mathrm{s}$ or more, and their dynamic interactions can lead to changes in plasma pressures, which can overwhelm the magnetic protection of the planet, as shown in a simulation depicted in Fig. 12 for solar wind at a speed of $1100 \mathrm{~km} / \mathrm{s}$.

Solar-Rotation Timescale Solar rotation should have important recurring heliospheric effects near Mercury. Coronal holes have lifetimes of several 11-y solar-activity cycles, so their associated fast solar wind streams are observed in subsequent solar rotations (the Sun has an 11-y sunspot and activity cycle and a 22-y full magnetic cycle for periodic reversal of the polarity of its magnetic field). CMEs have a tendency to erupt from restricted ranges of solar longitude. Given the 33.9-day average synodic solar rotation period with respect to Mercury and accounting for Mercury's orbital eccentricity, solar plasma features should affect Mercury with this periodicity.

Solar-Cycle Timescale Solar wind properties, such as density, speed, number and structure of CMEs, and composition, change during the 22-y solar magnetic cycle. For example, the He abundance increases from $<2 \%$ during solar maximum to $\sim 4.5 \%$ at solar minimum (Aellig et al. 2001), and the in-ecliptic solar wind transitions from purely slow wind at solar minimum to a mixture of the two types at maximum. However, the He abundance can exceed $10 \%$ in the closed field regions within coronal holes. The flux of anomalous and galactic cosmic rays detected at Earth depends on the solar cycle modulation, $\Phi$, which quantifies the energy lost by particles entering the heliosphere (Gleeson and Axford 1968). $\Phi$ is higher when the Sun is more active and the solar wind carries a more intense magnetic field through the inner heliosphere, and the flux of low-energy GCRs $\left(<10^{4} \mathrm{MeV} /\right.$ nucleon $)$ reaching Earth decreases (Beer et al. 2006). The intensity of GCRs in the most recent solar minimum exceeded that of the last five decades, indicating much lower solar activity (Mewaldt et al. 2010).

\subsubsection{Moderate-Timescale Variations}

Centurial Timescale Sunspots have been counted regularly by astronomers since the early 1600 s, creating a record of solar activity that spans four centuries. During times of increased sunspot numbers, solar activity is high; solar activity has increased, on average, over the last century. Periods of minimal solar activity occurred at the turn of the 18th, 19th, and 20th centuries, seemingly cyclic with a 90 -year period; there was a prolonged absence of sunspots in 1645-1715 during the Maunder Minimum (Pulkkinen et al. 2001). Beyond sunspot numbers, geomagnetic indices measured over the last century also indicate trends in solar activity (cf. Lockwood et al. 1999). These indices are used to derive the mean solar wind speed, under the assumption that speed is related to the expansion of open magnetic flux in the heliosphere (Wang and Sheeley 1990). Mean IMF strength and solar wind speed both apparently increased during the first half of the 20th century (Rouillard et al. 2007).

Millennial Timescale Cosmogenic radionuclides produced by the interaction of GCRs with Earth's atmosphere, such as ${ }^{10}$ Be measured in ice cores (Raisebeck et al. 1987; Sonett et al. 1987; Zank and Frisch 1999; Frisch and Slavin 2006) and ${ }^{14} \mathrm{C}$ measured in tree rings (e.g., Miyake et al. 2012), give a proxy record of solar activity going back at least 10,000 years. Longer timescales have been analyzed from meteorites (e.g., Eugster et al. 2006). 
These proxies show that $\Phi$ has varied by more than a factor of 3 and was lower than it is now $\sim 85 \%$ of the time. Solar activity was low about 7000 years before present (BP), rising to a peak at about 2000 years BP (Steinhilber et al. 2008). Space weathering by solar wind plasma is expected to increase when the solar activity is high, whereas cosmic ray access to the inner heliosphere increases when solar activity is low. Overall cosmic ray variability is not expected to vary by orders of magnitude. These timescales are within the range of timescales predicted by laboratory experiments of solar wind irradiation, which have been variously suggested to be $\sim 5000$ y (Loeffler et al. 2009) to $10^{4}-10^{6}$ y (Strazzulla et al. 2005; Vernazza et al. 2009).

\subsubsection{Long-Timescale Variations}

Galactic Timescales Theoretical and observational modeling studies show that the properties of the heliosphere, and therefore the interplanetary environment, are affected by the galactic environment of the Sun (e.g., Zank and Frisch 1999; Frisch and Slavin 2006). Although the Sun has resided in a low-density hot region (the Local Bubble, or LB) of the galaxy for more than 3 My, LB properties have varied. The Sun has encountered local interstellar clouds (LICs) within which densities, temperatures, and magnetic field properties differ (Frisch and Slavin 2006). Interactions of the heliosphere with the local interstellar medium (ISM) affect the solar wind and particle environment experienced by the planets. Simulations in which ISM properties were held constant to current values except for the density of interstellar hydrogen $\left(\mathrm{H}^{0}\right)$ (Zank and Frisch 1999) show that changing $\mathrm{H}^{0}$ density from current values of $\sim 0.2 \mathrm{~cm}^{-3}$ to $10 \mathrm{~cm}^{-3}$ reduced the size of the heliospheric cavity (the distance from the Sun to the upstream termination shock) from current values of $\sim 80-120$ AU to $~ 10-14$ AU. This change is due to charge exchange between the ISM and the solar wind at the heliopause, which leads to significant deceleration of the solar wind through momentum transfer (Zank and Frisch 1999). Zank and Frisch (1999) also demonstrated that the heliospheric configuration can become highly unstable, with the formation, disappearance, and re-formation of the termination shock recurring in cycles on a timescale of 280 days. Under current ISM conditions interstellar hydrogen is entirely ionized within a few AU from the Sun; however, in Zank and Frisch's (1999) simulation, the interstellar neutral hydrogen density at Earth changes from essentially zero to $\sim 2 \mathrm{~cm}^{-3}$, thus exposing Earth to bombardment by $\mathrm{H}^{0}$. There is also evidence for higher magnetic field strengths within denser LICs, which would further decrease the heliospheric radius by one-half to one-third, depending on the magnetic field configuration (Zank and Frisch 1999), increasing exposure of the inner planets to interstellar particles.

The Zank and Frisch (1999) simulation showed that pickup ions completely dominate the thermodynamic character of the outer heliospheric solar wind, creating solar wind temperatures in excess of the current $10^{5} \mathrm{~K}$. This higher temperature in turn creates an inward pressure gradient, causing the solar wind to decelerate much more than through mass-loading scenarios; thus density as a function of heliocentric distance no longer obeys the usual $r^{-2}$ dependence on solar distance $r$. (Mass loading is a process by which neutral atoms are ionized and picked up by the solar wind and accelerated to solar wind velocities. If the ions are heavier than the solar wind protons, then to conserve momentum the solar wind velocity decreases.)

Interactions with LICs also increase the cosmic-ray flux at 1 AU (Zank and Frisch 1999), in two ways: (1) the increased pick-up ion population increases the ACR population, and (2) GCRs are no longer as markedly modulated by an extended solar wind, which is shortened due to the reduction in the size of the heliospheric cavity (Zank and 
Frisch 1999). Evidence for past interactions of the Solar System with LICs has been inferred from Earth's geologic record. Spikes in ${ }^{10} \mathrm{Be}$ concentration in Antarctic ice core samples at 33,000 and 60,000 years BP have been attributed to an increase in cosmic-ray flux on Earth's atmosphere (Raisebeck et al. 1987; Sonett et al. 1987; Zank and Frisch 1999; Frisch and Slavin 2006). The increased cosmic-ray flux can be produced by changes in the Sun's galactic environment.

Though most studies of the effects of changes in the Sun's galactic environment have focused on the repercussions for Earth's climate, there will also be effects throughout the Solar System. The effects at Mercury's orbit have not been quantified, but the timescales are on the order of millions to billions of years, i.e., during the time of regolith formation for both Mercury and the Moon (Figs. 18 and 19). To understand the potential space weathering effects, the turnover rate of the upper regolith is needed. This effect should be averaged over the depth of gardening and would differ between rocks and soil.

Geologic Timescales Lunar regolith samples record solar wind impacts that potentially date to nearly $4 \mathrm{Ga}$ (billion years ago) and reveal the changing composition of the solar wind. The noble gases $\mathrm{Xe}$ and $\mathrm{He}$ are enriched in samples of ancient regolith breccias by at least factors of 2, whereas the ${ }^{3} \mathrm{He} /{ }^{4} \mathrm{He}$ ratio is $20 \%$ lower than more recent (1.5-3.5 Gy younger) soil samples (Kerridge et al. 1985). Given that Xe and ${ }^{4} \mathrm{He}$ must be accelerated by a stronger Coulomb drag (a component of solar-wind acceleration theory in which ionized hydrogen can preferentially accelerate heavier species, Wiens et al. 2004) than required by other noble gases (Geiss 1973), the compositional differences indicate that solar wind fluxes were higher in the past (Geiss 1974).

Additional clues about the Sun over geologic timescales come from studies of solaranalog stars (Ribas et al. 2005; Wood 2006; Güdel 2007; Guinan and Engle 2009). The Sun entered the main sequence at about $4.6 \mathrm{Ga}$. The bolometric luminosity at zero-age main-sequence (ZAMS) was $\sim 70 \%$ of its present output (Siess et al. 2000; Ribas et al. 2005), so the optical and infrared thermal outputs were $30 \%$ lower than today. However, the ZAMS Sun rotated faster than now. This faster rotation generated higher levels of magnetic activity and induced higher fluxes in UV, extreme ultraviolet (EUV), and X-ray outputs. Such photon emissions may have been factors of 10, 100, and 1000 higher than present levels, respectively (Ribas et al. 2005; Güdel 2007).

In addition, enhanced high-energy emissions and frequent flares typical of young stars also produce more powerful particle winds (Ribas et al. 2005). Although stellar winds from solar-analog stars have not been directly measured, indirect measurements through their interactions with the ISM (Wood et al. 2001, 2002; Ribas et al. 2005; Wood 2006) indicate the coronal mass-loss rates for several solar-analog stars. From these, Wood et al. (2002) proposed that the mass-loss rate of the Sun has decreased with time $t$ as $t^{-2}$, indicating that the solar wind flux may have been 1000 times greater than at present (Wood et al. 2002; Ribas et al. 2005). Young, solar-analog stars exhibit explosive, episodic releases of plasma, generating non-thermal, high-energy particles, similar to current solar CMEs, but hundreds of times stronger and more frequent (Audard et al. 2000; Ribas et al. 2005).

Estimates of solar output over time are compared with major geologic time periods and events from asteroids and the Moon in Figs. 18 and 19. These figures permit us to evaluate regolith exposure and alteration by solar processes described above (applicable to Mercury to the degree that we can tie Mercury's geologic history to the lunar timescale). These figures provide a picture of the potential solar wind processing experienced by the regolith early in both the Moon's and Mercury's history. This early history has implications for the depth to which the regolith has been weathered. Higher solar wind activity during early solar system 

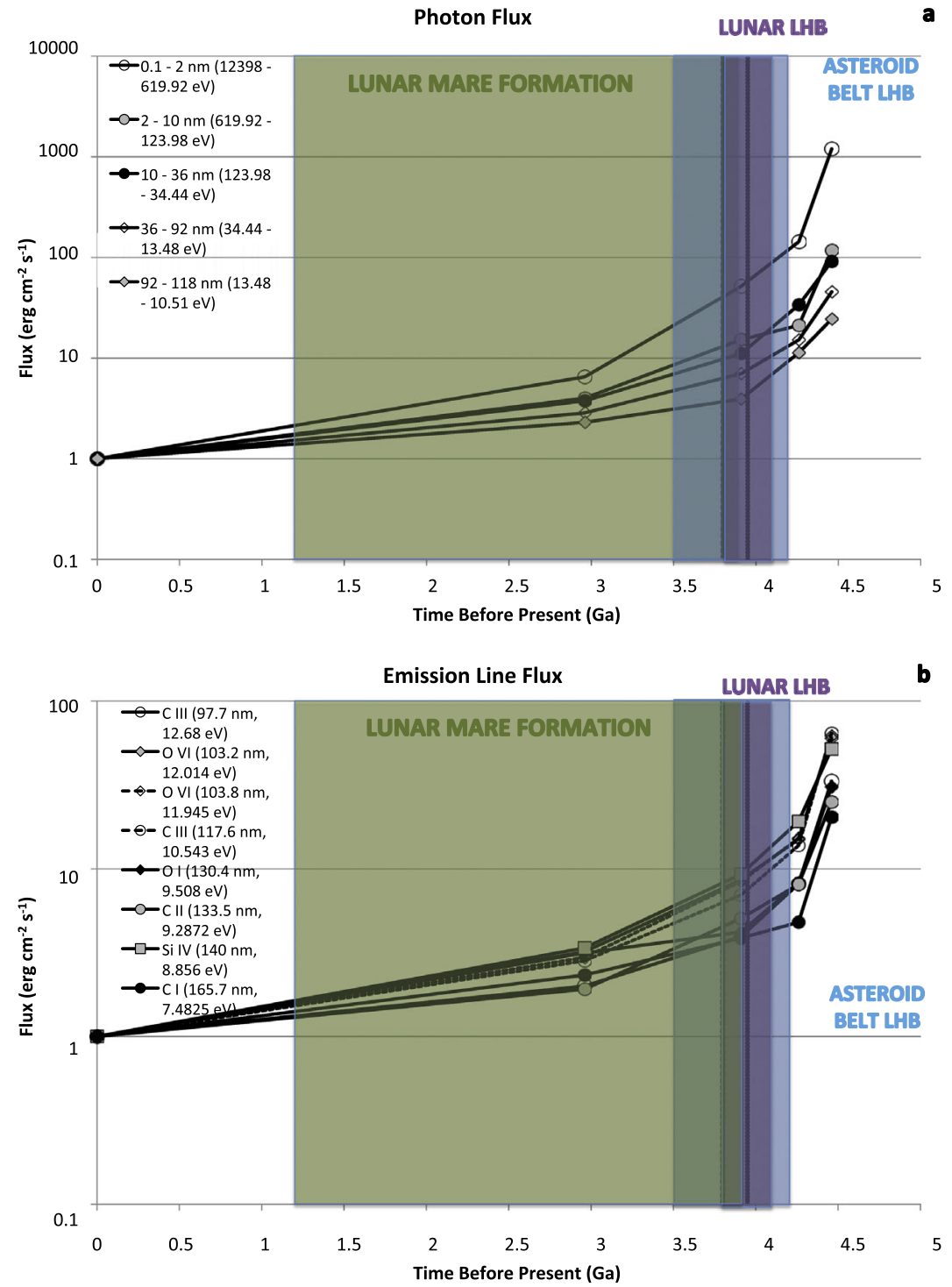

Fig. 18 Estimates of the flux of solar high-energy photons in (a) EUV to soft X-ray wavelengths and (b) near UV to EUV wavelengths versus time from studies of solar-analog stars at various ages (Ribas et al. 2005). The top graph plots the change with time in photon flux for wavelength (energy) ranges of 0.1-2 nm (open circles), 2-10 nm (gray circles), 10-36 nm (black circles), 36-92 nm (open diamonds), and 92-118 nm (gray diamonds). The bottom graph plots the change with time in the emission line flux of several atomic species (C I, C II, and C III plotted as black, gray, and open circles, respectively; O I plotted in black diamonds, the two O IV lines plotted in gray and open diamonds; Si IV plotted in gray squares). Also shown in both graphs are estimates of the time periods of the lunar LHB (purple region, 4.1 to 3.8 Ga, Chapman et al. 2007), asteroid main belt LHB (blue region, 4.2 to $3.5 \mathrm{Ga}$, Chapman et al. 2007), and lunar mare volcanism (green region, 3.9 to $1.2 \mathrm{Ga}$, Hiesinger et al. 2000, 2003). For comparison, the estimated time of the Caloris basin formation (dotted line) and Caloris plains formation (dashed line) are also plotted. These graphs show that during the time of regolith formation in the LHB era the solar photon output was much greater than at present for both energy regimes. Estimates given beyond the current age of the Sun are based on measurements of solar-analog stars older than the Sun 


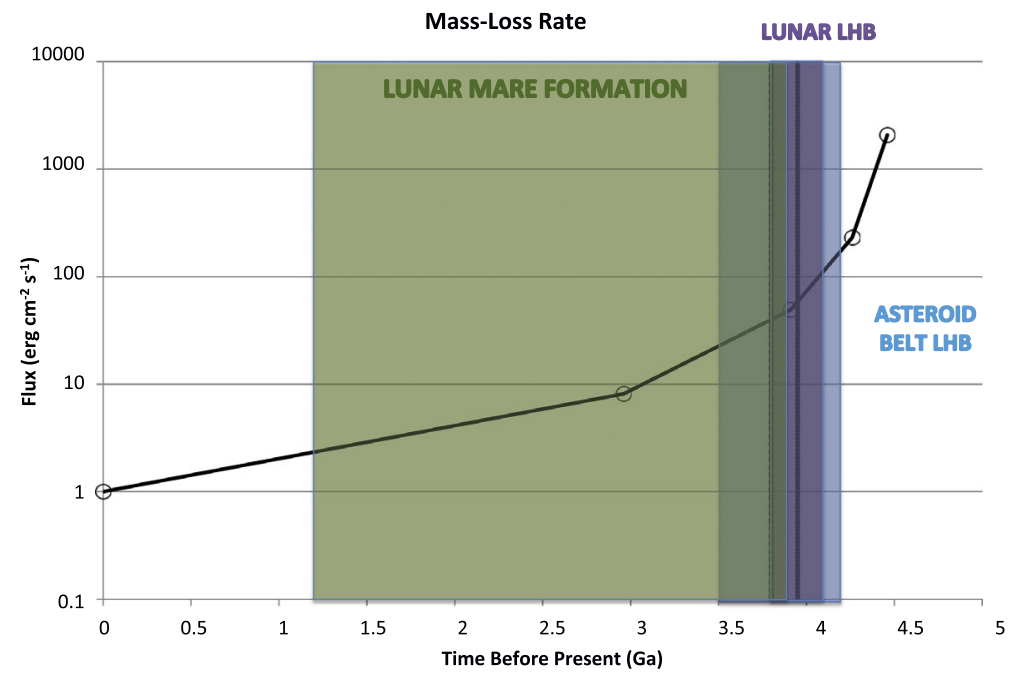

Fig. 19 Estimated solar mass loss rates (Ribas et al. 2005) compared with geologic time periods for the Moon and asteroid belt. Also shown are the estimated time periods of the lunar LHB (purple region, 4.1 to $3.8 \mathrm{Ga}$, Chapman et al. 2007), asteroid main belt LHB (blue region, 4.2 to $3.5 \mathrm{Ga}$, Chapman et al. 2007), and lunar mare volcanism (green region, 3.9 to $1.2 \mathrm{Ga}$, Hiesinger et al. 2000, 2003). For comparison, the estimated time of the Caloris basin formation (dotted line) and Caloris plains formation (dashed line) are also plotted. These graphs show that during the time of regolith formation in the LHB era the solar mass output was much greater than at present, comparable to or greater than that during CMEs in the modern era. Estimates given beyond the current age of the Sun are based on measurements of solar-analog stars older than the Sun

history implies a higher probability of weathered material at greater depths. The current elemental abundance measurements from MESSENGER show nearly uniform abundances down to tens of centimeter depths (Evans et al. 2012; Weider et al. 2012; Peplowski et al. 2014), a result consistent with a regolith that has been uniformly weathered to a least these shallow regolith depths.

There are several competing hypotheses regarding the impact bombardment history of the Moon. These theories are based on geochemical analyses of lunar samples, crater density measurements, and solar system dynamical evolution models. Lunar sample analyses show a clustering of radiometric impact ages at $\sim 3.9 \mathrm{Ga}$ (Papanastassiou and Wasserburg 1971a, 1971b; Wasserburg and Papanastassiou 1971; Turner et al. 1973), whereas laboratory analyses of lunar meteorites show a paucity of impact ages older than $\sim 4$ Gy (Cohen et al. 2000). These geochemical measurements are suggestive of a period of heavy bombardment in the Moon's impact history, often referred to as the lunar cataclysm or late heavy bombardment (LHB). Analyses of lunar crater densities have led to an interpretation of the Moon's impact history that does not require a lunar cataclysmic scenario. From an estimated age of $\sim 4.1 \mathrm{Ga}$ for the lunar Nectaris basin (derived from Apollo 16 highland sample analysis, e.g., Maurer et al. 1978), the density of craters on terrain with ages between 4.1 and 3.5 Ga appears to decline exponentially (Neukum and Wilhelms 1982; Neukum 1983; Neukum and Ivanov 1994). This exponential decay was extrapolated backward in time to estimate the impact flux during the Moon's earlier history to support a long smooth decay in the lunar impact flux (Neukum and Ivanov 1994; Morbidelli et al. 2012). However, it can be argued on the basis of Apollo 16 Descartes terrain samples that the age of the Nectaris impact is $3.9 \mathrm{Ga}$, in which case the same crater counting statistics would indicate a much steeper de- 
cline in the impact flux between 3.9 and 3.5 Ga (Ryder 1990, 2002; Stöffler and Ryder 2001; Morbidelli et al. 2012). Extrapolation of this steeper decline in impacts to earlier times leads to physically unrealistic implications, such as the Moon accreting more than a lunar mass since its formation (Ryder 2002; Morbidelli et al. 2012). Thus this age for Nectaris, combined with the crater statistics, implies that the impact flux was smaller before $3.9 \mathrm{Ga}$ than in the 3.9 to $3.5 \mathrm{Ga}$ time frame, consistent with the hypothesis of a late heavy bombardment (Morbidelli et al. 2012).

Crater size-frequency distributions show a change in the shape of the distribution between the highlands and the younger maria (Strom et al. 2005). Velocity calculations of the populations of projectiles impacting the Moon show a change between Nectarian and Pre-Nectarian times (Marchi et al. 2012). Both of these findings suggest a marked change in the impactor populations, in agreement with some versions of the lunar cataclysm hypothesis (e.g., Morbidelli et al. 2012). However, a study by Fassett et al. (2012) showed that a change occurred in the impactor population in the mid-Nectarian time frame, counter to the requirements in the lunar cataclysm hypothesis (Morbidelli et al. 2012). From dynamical models of solar system evolution modeling derived from the socalled "Nice model" (Morbidelli et al. 2005; Nesvorny et al. 2007; Charnoz et al. 2009; Broz et al. 2011), including the concept of an extended or "E-belt" of early asteroids (Bottke et al. 2012), in conjunction with the lunar cratering record and the geochemical constraints from lunar sample analyses, Morbidelli et al. (2012) showed that the lunar impact flux can be described by a sawtooth-like distribution: an exponential impact flux rate from 4.1 to 3.5 Ga consistent with the results of Neukum and Ivanov (1994) and a moderate increase in flux rate at 4.2 to $4.1 \mathrm{Ga}$ in agreement with the weak cataclysm scenario of Hartmann et al. (2000).

For comparison with the solar output, the following is assumed. The Nectaris basin formed on the Moon at 4.1-3.9 Ga, and the Imbrium basin at $~ 3.85 \mathrm{Ga}$ (Chapman et al. 2007; Stöffler and Ryder 2001). As many as 10 lunar basins may have formed between these two events. Orientale formed somewhat after Imbrium, but two-thirds of the recognized lunar basins formed before Nectaris (Chapman et al. 2007). Thus the LHB ended $~ 3.85$ to $3.8 \mathrm{Ga}$; it commenced before Nectaris formed, but how long before is uncertain $(4.1 \mathrm{Ga}$ is adopted in Figs. 18 and 19). [An LHB-like period in the asteroid belt apparently began somewhat earlier than on the Moon and also lasted longer, from $\sim 4.2$ to $3.5 \mathrm{Ga}$ (Chapman et al. 2007).] The period of lunar volcanism has been constrained by crystallization ages of basalts returned by Apollo missions, which range from 3.8 to $3.15 \mathrm{Ga}$ (Stöffler and Ryder 2001). Stratigraphic information and the size-frequency distributions of superposed impact craters indicate that mare volcanic units on the Moon span a much longer interval of ages, from $\sim 3.9 \mathrm{Ga}$ or earlier to $\sim 1.2 \mathrm{Ga}$ or younger (Hiesinger et al. 2000, 2003).

There are no samples to constrain ages of geologic features on Mercury. If the timing of the lunar LHB and the size-frequency distributions of the bombarding population on the Moon apply to Mercury (Marchi et al. 2009), then Mercury's Caloris basin formed late in the LHB, perhaps $\sim 3.95 \mathrm{Ga}$, and the major expanses of volcanic smooth plains interior and surrounding the basin formed somewhat later, perhaps $\sim 3.8 \mathrm{Ga}$. From a model of early lunar cratering chronology (Morbidelli et al. 2012) and dynamical extrapolation to Mercury by Marchi et al. (2009), the oldest geologic terrains on Mercury are estimated to have been emplaced between 4.1 to 4.0 Ga on the basis of crater statistics (Marchi et al. 2013).

Most of these ancient geologic events occurred when the Sun's high-energy output was much greater than now, and alteration of the surficial regolith by particle and photon bombardment processes must have been greater. However, it is unclear to what degree such surficial regolith processes may be discerned from periods during and before the LHB, or 
even during later periods of extensive volcanism. It is also unclear what volumetric fraction of near-surface materials on the Moon or Mercury may have been reworked (e.g., what fraction of upper crustal materials may have been depleted in volatiles or what fraction of $\mathrm{FeO}$ was converted to nanophase $\mathrm{Fe}$ ) and to what depth. Since impact basin formation and plains volcanism ended on the Moon and Mercury, the surficial regolith has been gardened to depths of meters (Heiken et al. 1991), as discussed above; only in isolated locations was surficial regolith formation punctuated by a large $(>10 \mathrm{~km}$ diameter) crater. Prior to the end of plains volcanism, multiple lava flows meters thick, cumulatively up to kilometers thick, were emplaced, a substantial fraction of which (depending on individual flow thicknesses and time intervals between flows) were not processed markedly by surface exposure and regolith gardening. Until late in the LHB, near-saturation of the surfaces of Mercury and the Moon by giant impact basins and their extensive ejecta blankets and sculpturing formed megaregoliths kilometers to tens of kilometers in thickness. If the size-frequency distribution of ancient meteoroids and micrometeoroids was similar to that of today, the immediate surfaces of these volcanic and impact deposits were presumably churned to depths of centimeters to meters, but the bulk of the volcanic deposits and megaregolith was never located within millimeters of the surface and thus was not subject to solar processing. The shallowly sloping size-frequency distribution of craters $>10 \mathrm{~km}$ diameter indicates that the near-surface area of the Moon and Mercury are dominated by individual large cratering impacts, so the megaregolith has not been repeatedly churned in the manner of the surficial regolith. The latter has been gardened by much smaller impacts characterized by a steeply sloping size-frequency distribution (Chapman et al. 2007).

The comparisons in Figs. 18 and 19 show that the solar photon flux was substantially higher over a range of wavelengths during the LHB when the regolith, especially at meter to kilometer scales, was being formed. During this period the optically active portion of the regolith (millimeters deep) was also being generated and overturned to substantial depths. Lunar regolith samples show evidence for this period of higher solar activity (Geiss 1974; Kerridge et al. 1985). Thus the surfaces of both objects have been subjected to solar-induced weathering processes during their regolith formation periods, indicating in particular that space weathered regolith is present to depths sampled by MESSENGER's XRS and GRS. We must await quantitative modeling of these regolith processes to estimate the fraction of upper crustal material that has been processed. Perhaps rare regolith breccias from the Moon (and even Mercury, if relevant meteorites from Mercury are found) can provide insight to ancient events.

\subsection{Connections to Space Weathering Processes}

Solar wind and solar irradiation effects may be characterized by composition, energy, and flux (Table 7) for the Moon, Mercury, and main-belt asteroids (nominally 2.7 AU solar distance). These effects are all associated with surface alteration processes. Although particle energies are similar across this region of the Solar System, impact energies are not equivalent. For example, protons are accelerated when they cross Mercury's cusp regions (Massetti et al. 2007). Lunar impact energies of $\sim 1 \mathrm{keV}$ have a corresponding energy of $\sim 3-7 \mathrm{keV}$ on Mercury (Massetti et al. 2007). Proximity to the Sun exposes Mercury's surface to 5.2 to 11.9 times more solar flux at perihelion and aphelion, respectively, than at the Moon. These effects translate to the following differences:

- Higher maximum temperatures and greater range of temperature on Mercury $[\sim 100$ to $700 \mathrm{~K}$ (Morrison 1970; Davies et al. 1978)] than on the Moon [ 100 to $400 \mathrm{~K}$ (Keihm and Langseth 1973; Chase et al. 1974, 1976)]. 


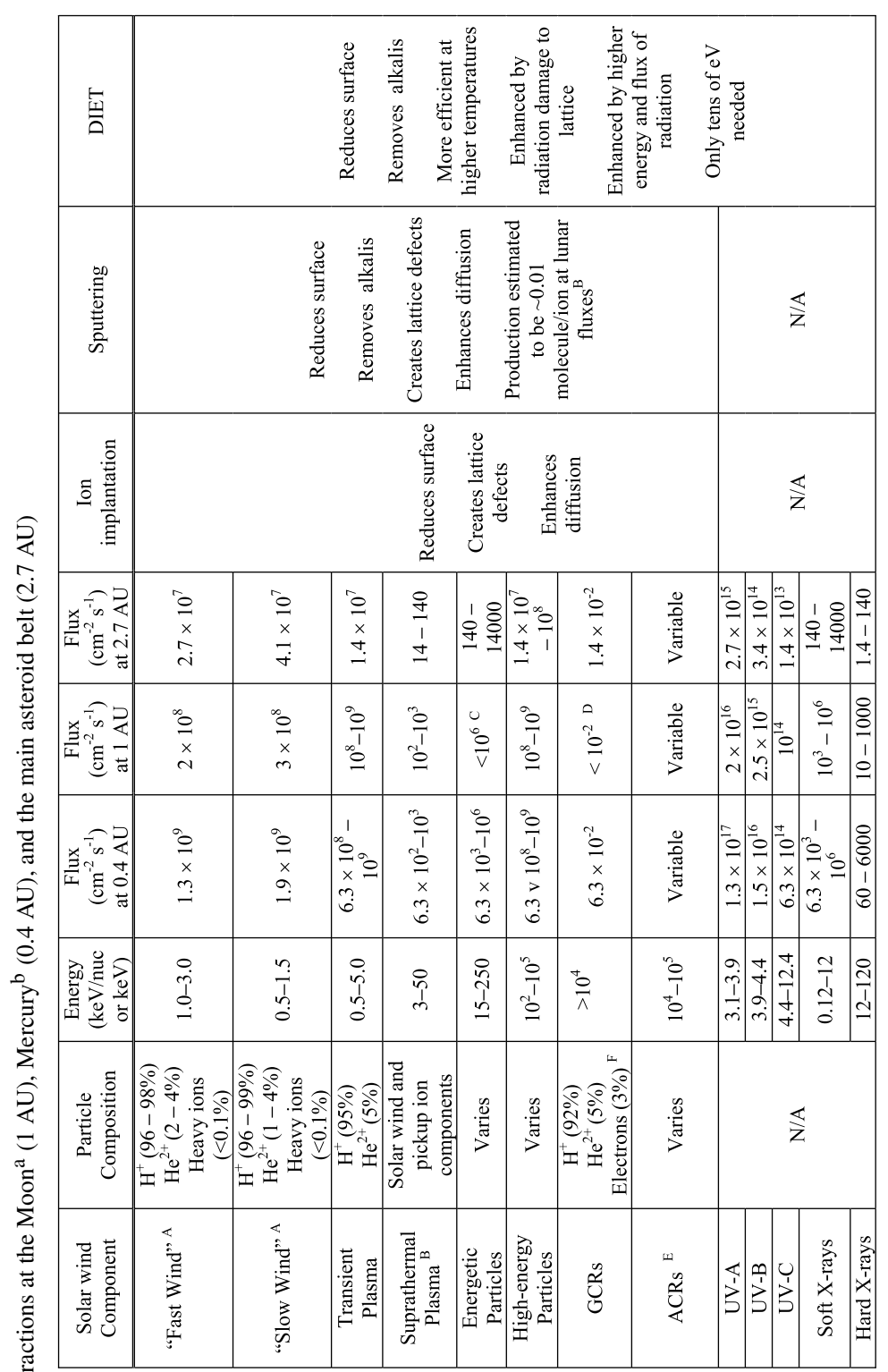

핳

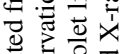

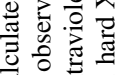

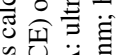

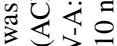
可 要安的

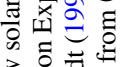

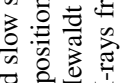

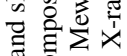
芶记芒窟

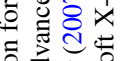

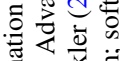

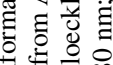
记

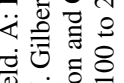

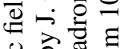
을 용

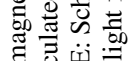

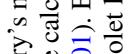
응 흐응

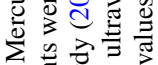
ह

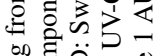
음 ํㅜㅇ 융 굴 0

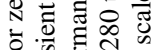

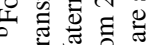

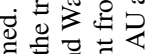

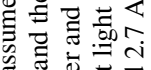
की

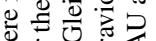
记

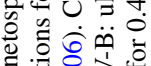
on

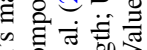
= 可泮 $\Xi$ 氙

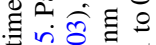
ช 0 웅.

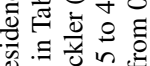
踏

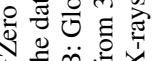


- Higher particle and photon fluxes than at the Moon (by a factor of $1 / r^{2}$, or 5.2-11.9).

The higher temperatures affect micrometeoroid impact and DIET processes. Cintala (1992) calculated that the ratio of pure liquid produced during impact events at $100 \mathrm{~K}$ versus $700 \mathrm{~K}$ is $1: 1.3$, implying a $30 \%$ greater production of melt products, such as agglutinates, on Mercury's dayside versus nightside. A similar calculation for pure vapor production yielded a ratio of 1:1.15, implying $15 \%$ more vapor products, such as grain patinas, on the dayside than the nightside. Differences in impact velocity between the Moon and Mercury contribute more to the variations in impact melt and vapor production, but temperature is also a non-negligible contributing factor. ESD experiments show linear increases in ion yields with increasing temperatures in the 350-550 K range (Yakshinskiy and Madey 2004, 2005; McLain et al. 2011).

Each of the processes listed in Table 7, along with micrometeoroid bombardment, acts to chemically reduce the regolith grains. The 5.2 to 11.9 higher solar radiation fluxes and the order of magnitude higher micrometeoroid flux all work to make Mercury's surface environment, for a given initial composition, more highly reducing than those of the Moon or asteroids. Even over galactic and geologic timescales (timescales spanning the generation of the regolith), Mercury's environment has been more reducing than the lunar environment. Even if Mercury's initial composition was chemically reduced, as suggested by its high surface $S$ and low surface Fe contents (Nittler et al. 2011), the space environment (under current conditions or conditions existing over the timescales of regolith generation) works to remove volatiles at a more efficient rate on Mercury than on the Moon or asteroids. However, the measured abundance of such volatiles as S, Na, and K (Evans et al. 2012; Peplowski et al. 2012; Weider et al. 2012) indicates that the surface also provides efficient repositories or sinks for these species, suggesting that the composition of the surface may be highly recycled. These species may be re-claimed within the regolith as adsorbates, constituents in grain vapor coatings or patinas, or an impact glass fraction of the soil. Meteoroids also contribute an exogenic component of volatile material (e.g., Noble et al. 2007). Spaceweathering processes can be assumed to enrich the surface of Mercury in refractory species and low-sputtering-yield elements such as Fe and Ti (McCord et al. 2001) with the possibility of high volatile-content grain coatings. Models of the surface source for observed exospheric abundances of $\mathrm{Na}$ show that the recycling of removed $\mathrm{Na}$ is not necessary to match the Na content over time. Killen et al. (2007) examined the diffusion rates of $\mathrm{Na}$ within regolith grains, coupled with regolith gardening (overturn) rates, and demonstrated that the exospheric sodium observed can be supplied by the surface via PSD even if the Na is lost to the system once removed from the regolith.

\section{Mercury's Surface}

We know about Mercury's surface properties from analyses and modeling of Earth-based reflectance and emission spectra, radar observations, and spacecraft imaging, elemental remote sensing, and spectral measurements. Modeling efforts constrain physical characteristics, such as porosity and grain size, and compositional properties, such as elemental abundances and mineral identifications. As discussed above, regolith porosity and grain size affect diffusion rates and trapping efficiencies, key moderators of space weathering processes. Surface composition also affects space-weathering rates, and in turn is altered by these same processes as evidenced by space-weathering-induced spectral changes. In this section we review constraints on the physical character and composition of Mercury's regolith and the observable effects of space weathering. 


\subsection{Regolith Characteristics}

Porosity, grain size distribution, surface roughness, and grain albedo and structure are constrained by analysis and modeling of photometric measurements. Reflectance measurements as a function of incidence, emission, and phase angles examined with Hapke's (1981, 1984, 1986, 2002) model can provide measures of regolith grain characteristics that can be compared among solar system bodies. Although it has been demonstrated for physically characterized laboratory samples that many of the parameters in Hapke's model represent a combination of physical properties (Shepard and Helfenstein 2007), it is still useful to compare modeling results between surfaces. For example, modeling of the opposition effect (the surge in reflectance at phase angles near $0^{\circ}$ ) provides insight into the correlations between grain size and regolith porosity. With Hapke's model and the assumption that Mercury's surface regolith is dominated by impact generation, Domingue et al. (2010) derived a correlation between possible grain sizes and regolith porosity for Mercury. For an impact-generated regolith, comminution creates a grain-size characterization parameter, $Y$, given by

$$
Y=\frac{\sqrt{3}}{\ln \left(r_{l} / r_{s}\right)}
$$

(Bhattacharya et al. 1975), where $r_{l}$ and $r_{s}$ are the radii of the largest and smallest grains, respectively. The relationship between $Y$ and the opposition width parameter, $h$, in the Hapke model is given by

$$
h=-\left(\frac{3}{8}\right) Y \ln (\rho),
$$

where $\rho$ is the porosity of the optically active top layer. Porosity estimates over a range of grain-size ratios, taken from the photometric analyses of Domingue et al. (2010) of the ground-based observations of Mallama et al. (2002), MESSENGER imaging data, and comparable lunar and S-type asteroid phase curves (Helfenstein and Veverka 1987, 1989; Hartmann and Domingue 1998), are shown in Fig. 20. Domingue et al. (2010) argued that although the absolute value of the porosity cannot be derived from the model parameter $h$, the results do indicate relative porosities among planetary surfaces of similar albedo, showing that Mercury's surface is less porous than either lunar or S-type asteroid surfaces, perhaps due to Mercury's higher surface gravitational acceleration or a larger component of fine soil particles $(<1 \mu \mathrm{m})$ that fill voids.

Application of Hapke's equations provides a method to compare grain-scattering properties with grain internal structures. In Fig. 20 the single-particle scattering function parameters (scattering amplitude, $b$, and scattering partition factor, $c$ ) derived for Mercury, the Moon, and several asteroids are compared with laboratory measures of these parameters for particles with known internal structural characteristics (McGuire and Hapke 1995; Domingue et al. 2011). The grains of all three bodies show similarities to particles with moderate to high density of internal scatterers (boundaries within the grain at which the index of refraction changes, such as cracks, vesicles, mineral inclusions, or rims and patinas). For Mercury there is an indication of an increasing density of internal scattering centers with decreasing wavelength, possibly constraining the sizes of these centers (Domingue et al. 2011). Domingue et al. (2011) postulated that if these centers are created through weathering processes, then there is a lower density of structural boundaries in regolith grains on Mercury than on asteroids and comparable densities on Mercury and the Moon. This inference was based on comparisons between the laboratory study results of McGuire and Hapke 

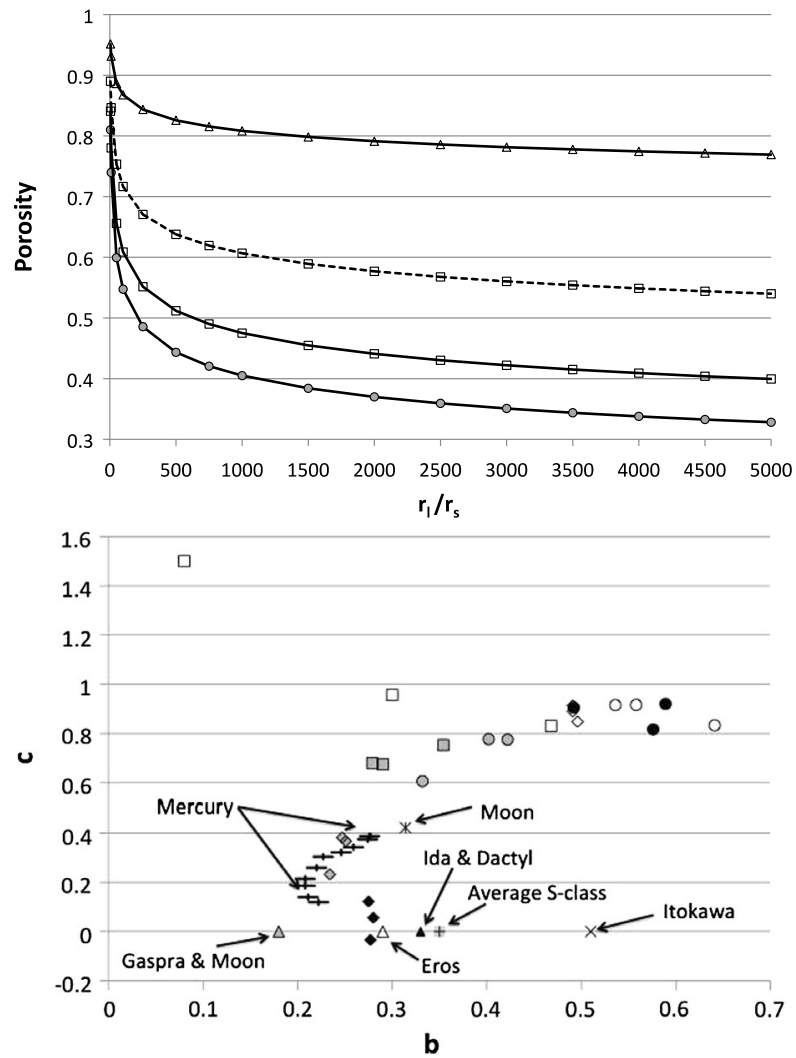

Fig. 20 Surface characteristics on Mercury and other bodies derived from photometric modeling. (Top) Variations in porosity as a function of the ratio of largest to smallest regolith grain for Mercury (filled circle), the Moon [open squares: solid line from Helfenstein and Veverka (1987); dashed line from Hartmann and Domingue (1998)], and average S-type asteroid [open triangle from Helfenstein and Veverka (1989)]. These are relative porosities and not absolute measurements. From Domingue et al. (2010). (Bottom) Relationship between photometrically derived scattering parameters for a Henyey-Greenstein scattering function $(b$ is the amplitude of the forward or backward scattering directions, and $c$ is the partition between forward and backward scattering) for Mercury (with uncertainties), the Moon, asteroids, and laboratory particles with a variety of physical characteristics. From Domingue et al. (2011). Open, gray, and black circles denote smooth, rough, and pitted spheres with no internal structures, respectively. Open, gray, and black diamonds represent spheres with low, moderate, and high densities of internal scatterers, respectively. The open squares represent irregular-shaped particles, and gray squares denote agglutinate particles. Lunar values from Hartmann and Domingue (1998) are shown with an asterisk. The open, gray, and black triangles indicate the asteroids Eros, Gaspra, and Ida, respectively. Values for Dactyl are identical to those for Ida (black triangle), and lunar values from Helfenstein and Veverka (1989) are identical to those for Gaspra (gray triangle). Itokawa is represented by a plus sign, and average S-type asteroids are represented by a gray square with a plus sign. The Mercury values plot in the region intermediate between particles with high and moderate densities of internal scatterers and in areas of increasing scattering-center density with decreasing wavelength

(1995) and the surface modeling results of Domingue et al. (2011). These boundaries have a size distribution such that a substantial portion has dimensions of $400 \mathrm{~nm}$ or less (Domingue et al. 2011).

Grain sizes have also been estimated by comparing spectral data with laboratory measurements. Hapke models of visible to near-infrared ground-based observations of Mercury 
indicate that much of the regolith has grain sizes near $\sim 20 \mu \mathrm{m}$ (Warell et al. 2010), but comparison of mid-infrared data with laboratory spectra indicate that regions on Mercury have grain sizes of 40 to $250 \mu \mathrm{m}$ (Sprague et al. 2009). Both of these grain size estimates are derived from comparisons with mineral spectra acquired at room temperature, however, rather than the high surface temperatures experienced at Mercury.

The Hapke surface roughness parameter (a measure of average surface tilt on scales ranging from a few tens of micrometers to the resolution footprint of the observation detector) for Mercury varies between $8^{\circ}$ and $16^{\circ}$ at $550 \mathrm{~nm}$ wavelength (Mallama et al. 2002; Warell 2004; Domingue et al. 2010, 2011). These values are lower, implying a smoother surface, than the $20^{\circ}-33^{\circ}$ values derived for the Moon (Helfenstein and Veverka 1987; Hartmann and Domingue 1998) and asteroids (Helfenstein and Veverka 1989; Helfenstein et al. 1994, 1996; Domingue et al. 2002; Lederer et al. 2005). Domingue et al. (2011) interpreted these roughness parameter values to indicate surface texture differences among these planetary objects on the scale of regolith grains, postulating that finer-grained dust on Mercury might clump together to produce conglomerate particles for which the grain boundaries act as scattering centers and increase the scattering-center densities. The clumping of fine particles to form conglomerates (via charging) would also decrease the average surface tilt at the scale of several grains, thus reducing the surface roughness value. Radar backscatter data, too, are influenced by surface roughness, in addition to topography and dielectric properties. Doppler spectra and depolarized radar images at $12.6 \mathrm{~cm}$ confirm that Mercury has a regolith and indicate a surface roughness value (average surface tilt on the scale of the radar wavelength) of $4^{\circ}$ in the smooth plains regions (Harmon 2007; Harmon et al. 2007), slightly smoother than the low values derived from photometry at visible to near-infrared wavelengths.

Mercury's cratered surface grossly resembles that of the Moon (Murray et al. 1974), in that both surfaces have been dominantly shaped by impacts. Lunar soils average $25-30 \%$ agglutinates, 3-5\% impact glasses, and 1-4\% material of meteoritic origin (Haskin and Warren 1991). Examination of mature lunar soils show they contain 40-60\% agglutinates (McKay et al. 1972), though how representative the returned soil samples are of the entire lunar regolith can be debated. Extrapolation to Mercury must take into account the generally higher impact speeds and greater impact flux. As discussed above, more melt and vapor are produced by impacts on Mercury than on the Moon. Because mature lunar soils contain up to $60 \%$ agglutinates (McKay et al. 1991), Mercury's regolith, at some locations, may have been converted entirely to glass (Cintala 1992). Cintala (1992) postulated that Mercury's regolith may retain little crystalline material. As noted above, the higher impact flux implies that the meteoritic component of Mercury's regolith is also higher (5-20 wt\%, Noble et al. 2007) than on the Moon, possibly contributing 1-5 wt\% FeO to Mercury's regolith (Noble et al. 2007), compatible with current estimates of the total Fe content.

Volcanic glass spherules are found in lunar soils (e.g., Delano 1986), and numerous lunar pyroclastic deposits (fragmented rock formed by explosive volcanism) have been mapped and analyzed with photogeological and other remote-sensing techniques (e.g., Gaddis et al. 2003). Deposits with anomalous color, surface reflectance, and morphology consistent with pyroclastic emplacement are also found on Mercury (Head et al. 2008, 2009; Murchie et al. 2008; Kerber et al. 2009, 2011). Therefore, explosive volcanic products such as pyroclastic beads and disrupted rock fragments are likely to be contained in Mercury's regolith.

Mercury's surface displays widespread evidence for large-scale deformation (e.g., Watters et al. 2009), including such tectonic features as lobate scarps, high-relief ridges, wrinkle ridges, and graben. Comminution unrelated to impact cratering, e.g., fault gouge and/or cataclasites (rock comminuted by high mechanical stress) created through frictional action 


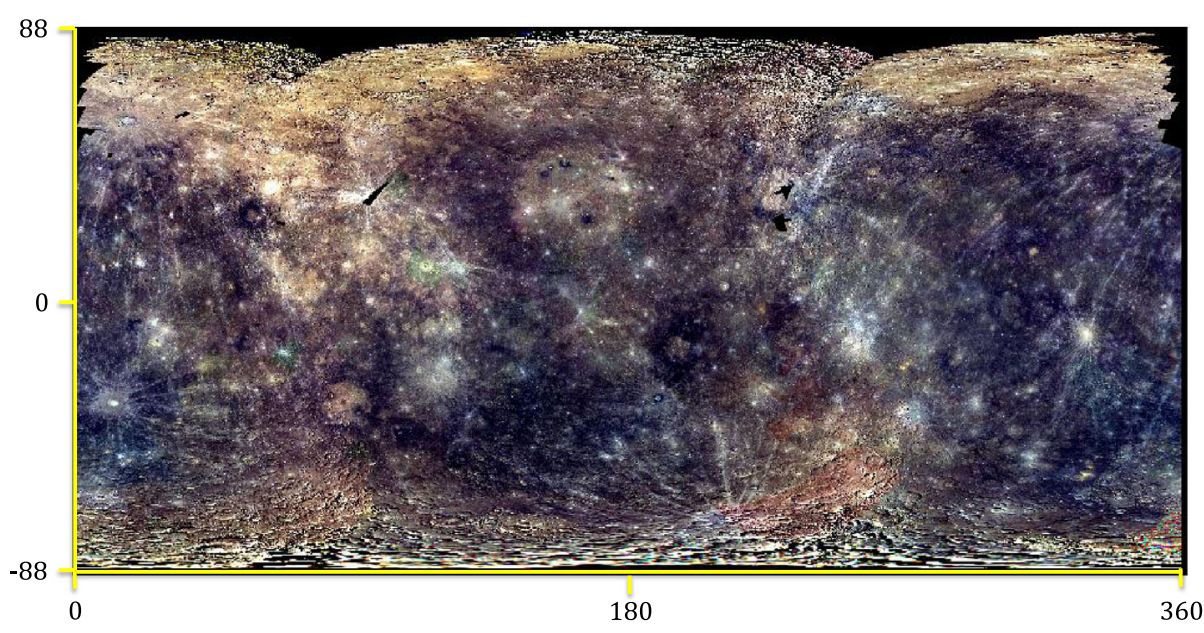

Fig. 21 Global color mosaic of Mercury's surface from MDIS images acquired during MESSENGER's primary orbital mission. This image has been stretched to enhance color differences. The red, green, and blue channels represent images acquired with the filters centered at 996.2-nm, 748.7-nm, and 433.2-nm wavelength. The red channel has been stretched to 0.069-0.128, the green channel to 0.056-0.109, and the blue channel to 0.033-0.070. Image seams are visible within the mosaic where images were acquired at high incidence and/or emission angles. The central longitude of the simple cylindrical projection is $180^{\circ} \mathrm{E}$

along fault planes, might contribute somewhat to Mercury's regolith. Downslope mass wasting affects the lunar regolith and probably also does so on Mercury, possibly enhanced by shaking during impact events and seismic slip on major fault structures.

\subsection{Geological Units}

Three major terrain types on Mercury can be defined on the basis of relative surface reflectance, spectral slope, and morphology (Robinson et al. 2008; Denevi et al. 2009) as determined from global images obtained with MESSENGER's Mercury Dual Imaging System (MDIS) (Hawkins et al. 2007). These units include smooth plains, intermediate terrain (IT), and low-reflectance material (LRM). The smooth plains, distinguished morphologically by a level to gently rolling surface and a lower density of impact craters, include three subtypes on the basis of their average reflectance and spectral slope: high-reflectance red plains (HRP), intermediate plains (IP), and low-reflectance blue plains (LBP) (Denevi et al. 2009). Intermediate terrain consists of regions with a higher density of craters than the smooth plains and have reflectance and color properties similar to the global mean within moderate variation (Denevi et al. 2009). The third major terrain, LRM, is distinguished by reflectance as much as $30 \%$ lower than the global mean, with spectral slopes $5 \%$ lower than HRP and no distinctive morphology (Denevi et al. 2009). Smooth plains cover $\sim 27 \%$ of Mercury's surface, and at least two-thirds of these smooth plains units are volcanic in origin (Denevi et al. 2013).

Color variations across Mercury's surface are showcased in the global color mosaic (Fig. 21) constructed from MESSENGER's orbital mapping campaign. Other spectrally distinct units identified on Mercury include fresh crater ejecta (FE), bright crater-floor deposits (BCFDs), and red spots (RSs) (Robinson et al. 2008). High-resolution images of Mercury's surface acquired from orbit (Fig. 22) show that the BCFDs include one or more depressions or hollows (Blewett et al. 2011), and the red spots are pyroclastic deposits (Kerber 
Fig. 22 (Top) This region of the 559-nm monochrome mosaic of Mercury's surface is centered on Basho crater (white arrow) at $32.4^{\circ} \mathrm{S}, 189.5^{\circ} \mathrm{E}$ (simple cylindrical projection). Basho crater is $74.6 \mathrm{~km}$ in diameter and displays bright ejecta rays with a dark halo of LRM ejecta close to the crater rim. The bright regions along the interior of the crater walls are hollows, shown in more detail in the bottom image (not reprojected). Also visible in this region is Tolstoj basin (black arrow) at $16.2^{\circ} \mathrm{S}, 195.3^{\circ} \mathrm{E}$. The basin is $355.6 \mathrm{~km}$ in diameter, and its interior is filled with smooth plains surrounded by LRM
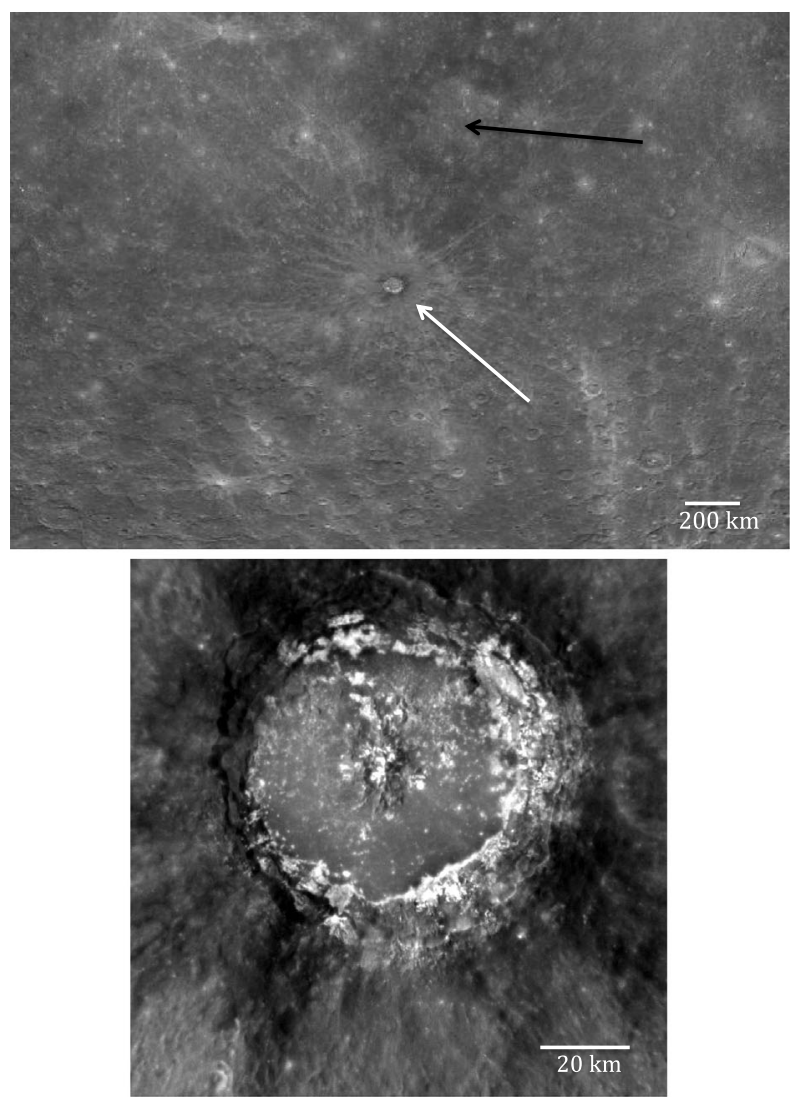

et al. 2011). Hollows are fresh-appearing, irregular, shallow, rimless depressions ranging from tens of meters to a few kilometers in size (Blewett et al. 2013). Hypothesized formation mechanisms for hollows involve the recent loss of volatiles through some combination of sublimation, outgassing, pyroclastic volcanism, and space-weathering processes (Blewett et al. 2013).

\subsection{Surface Composition}

Until MESSENGER completed its primary orbital mission, knowledge of Mercury's surface composition was limited to information inferred from ground-based reflectance observations, color imaging observations from Mariner 10 and MESSENGER flybys, and limited constraints on average elemental composition from flyby observations with MESSENGER's Gamma-Ray and Neutron Spectrometer (GRNS) instrument. Elemental information from the MESSENGER XRS and GRNS instruments has substantially improved since the spacecraft was inserted into orbit about Mercury, but the identification of specific surface minerals from MDIS and MASCS spectral observations remains elusive. All of this information provides an important context for a discussion of space weathering effects.

It has long been recognized that Mercury's surface is low in $\mathrm{FeO}$, on the basis of groundbased observations at microwave wavelengths (Mitchell and de Pater 1994), visible-nearinfrared reflectance spectra (Vilas and McCord 1976; McCord and Clark 1979; Vilas 1985; 
McClintock et al. 2008), and identification of minerals from thermal infrared Restrahlen bands (Sprague et al. 2009). Such low amounts of surficial Fe contrast with Mercury's bulk composition and large Fe-rich core, as inferred from its high density (Siegfried and Solomon 1974; Solomon 1976). Mercury's surface albedo, for both mature and immature terrains, averages 10-15\% darker than the lunar nearside (Warell 2004; Denevi and Robinson 2008; Warell et al. 2010), which has a strong bimodal albedo distribution (highlands and maria) that is not seen for the spectral units identified on Mercury. Denevi and Robinson (2008) found that young, Kuiperian-age rayed craters on Mercury are 30-50\% darker than analogous Copernican-age rayed craters on the Moon. These differences may mean that Mercury's regolith is composed of inherently darker material, that darkening processes have progressed further on Mercury than on the Moon, or both.

We first consider the evolution in our understanding of iron and titanium abundances on Mercury's surface (from both elemental and mineralogical perspectives). We then discuss plausible regolith darkening agents, temperature effects, and constraints on silicate, oxide, sulfide, and glass contents.

\subsubsection{Iron and Titanium}

The abundances of Fe and Ti on Mercury's surface are key to deciphering Mercury's formation and the origin of its high bulk metal fraction. Fe and $\mathrm{Ti}$ can be present in a variety of mineral forms including silicates, oxides, sulfides, and native metals. When $\mathrm{Fe}$ is bound to $\mathrm{O}$ in a silicate lattice (e.g., in olivine or pyroxene), an electronic transition in response to incident light produces a diagnostic spectral reflectance absorption band at $\sim 1 \mu \mathrm{m}$ wavelength. This feature is generally absent in whole-disk spectra of Mercury (Vilas 1985; McClintock et al. 2008, 2009) and in disk-resolved MASCS spectra (McClintock et al. 2008, 2009). Warell et al. (2006) reported a shallow absorption feature from 0.8 to $1.3 \mu \mathrm{m}$ (centered at $1.1 \mu \mathrm{m}$ ) in two disk-resolved observations, suggesting that iron-bearing silicates may be present but localized (Warell et al. 2006; Boynton et al. 2007). The global spectral coverage obtained by MASCS from orbit (Fig. 23), however, shows no evidence of a 1- $\mu$ m absorption feature (Izenberg et al. 2012, 2014; Klima et al. 2013). Spectral mixing models applied to ground-based mid-infrared measurements yield some solutions that contain hedenbergite $\left(\mathrm{FeCaSi}_{2} \mathrm{O}_{6}\right)$, a calcium-iron pyroxene, at the $\sim 30 \%$ level (Sprague et al. 2009). At this concentration, however, this mineral should also produce a 1- $\mu \mathrm{m}$ feature at NIR wavelengths, which is not seen in MASCS observations. Results from spectral mixing models, however, are constrained by the mineral spectral libraries to which the observations are compared.

MESSENGER reflectance spectra at near-ultraviolet and visible wavelengths show a downturn from the visible into the near-ultraviolet that has been attributed to oxygen-metal charge transfer (OMCT) (McClintock et al. 2008). This feature is exhibited only by transition metals (Burns 1993), the most abundant of which in the Earth-Moon system is iron. McClintock et al. (2008) suggested that the absorption edge seen in MESSENGER spectra is due to low-abundance Fe-bearing silicates; limits on equivalent $\mathrm{FeO}$ derived from reflectance spectra range from $\sim 3 \mathrm{wt} \%$ (Blewett et al. 1997) to less than $2 \mathrm{wt} \%$ (Warell and Blewett 2004; McClintock et al. 2008). Studies of the near-UV feature in the global MASCS data set coupled with the non-detection of a $1 \mu \mathrm{m}$ feature limits the $\mathrm{FeO}$ content to less than 2-4 wt\% (Izenberg et al. 2012, 2014). More detailed comparisons of MASCS data with laboratory spectra of low-Fe silicates indicate an $\mathrm{FeO}$ abundance of $<1 \mathrm{wt} \%$ (Klima et al. 2013). A comparison of Hapke models of MASCS spectra from MESSENGER's first Mercury flyby with laboratory mineral spectra yield between 1 and $8 \mathrm{wt} \% \mathrm{FeO}$ (Warell et al. 


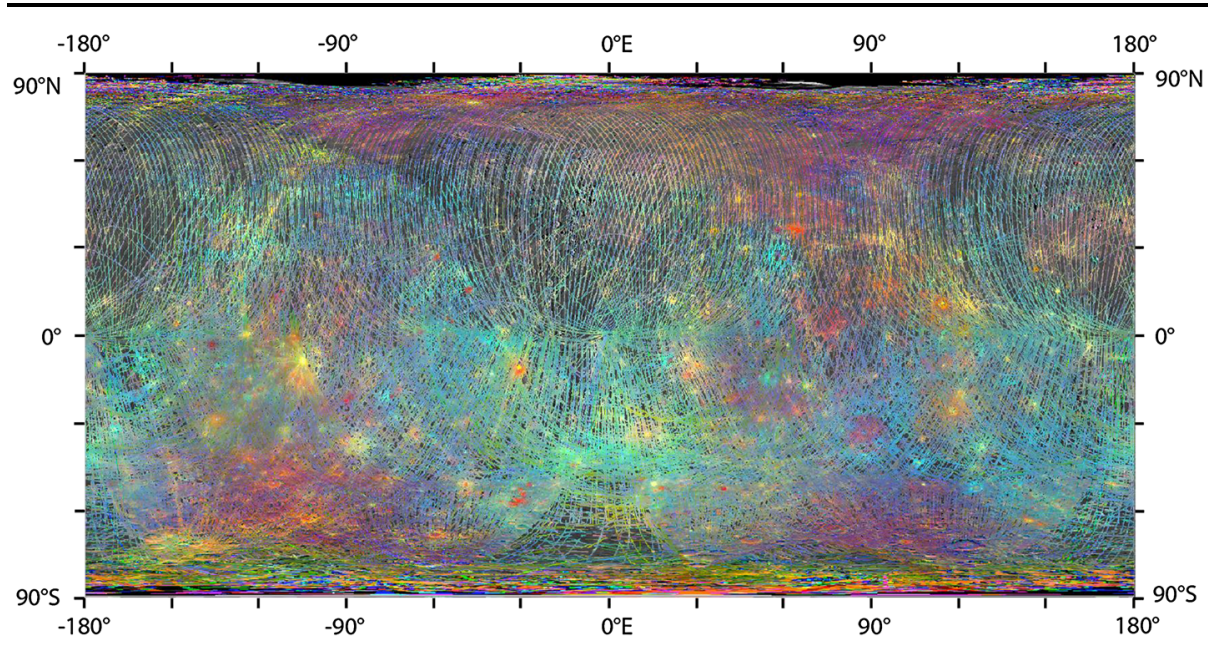

Fig. 23 Global coverage of MASCS Visible and Infrared Spectrograph (VIRS) observations acquired during MESSENGER's orbital mission phase. The red channel represents the surface reflectance at $575 \mathrm{~nm}$ (stretched to values of 0.03 to 0.1 ), the green channel represents the $415 / 750 \mathrm{~nm}$ spectral ratio (stretched to values of 0.46 to 0.64 ), and the blue channel represents the $310 / 390 \mathrm{~nm}$ spectral ratio (stretched to values of 0.53 to 0.67 ). The MASCS data overlay an MDIS monochrome mosaic with a central longitude of $0^{\circ} \mathrm{E}$. Caloris basin lies on the upper right-hand edge of this map (compared with the upper center of the maps shown in Fig. 21). The central longitude of the simple cylindrical map is $0^{\circ} \mathrm{E}$

2010), depending on modeled mineral combinations. A compilation of the spectral properties of example geologic units (Fig. 24) demonstrates that the OMCT feature in the UV is predominantly associated with younger surface units (Izenberg et al. 2014). This inference is consistent with the conversion of $\mathrm{FeO}$ to $\mathrm{npFe}^{0}$ in older, more mature regions. The lack of the diagnostic spectral reflectance absorption band at $\sim 1 \mu \mathrm{m}$ is also evident in the representative spectra in Fig. 24.

$\mathrm{Ti}$ substitutes for $\mathrm{Fe}$ in ferromagnetic minerals, such as iron oxides and spinels. It will also substitute for $\mathrm{Fe}$ in some pyroxenes. Its most common mineral form, however, is in an oxide such as ilmenite $\left(\mathrm{FeTiO}_{3}\right)$. The $\mathrm{Ti}$ concentration expressed as $\mathrm{wt} \% \mathrm{TiO}_{2}$ has been inferred to be quite low from ground-based NIR and microwave observations of Mercury (Mitchell and de Pater 1994; Warell and Blewett 2004). Blewett et al. (1997) evaluated the spectral effects of ilmenite on visible-NIR telescopic spectra and inferred a $\mathrm{TiO}_{2}$ abundance of $\sim 1 \mathrm{wt} \%$ or less, confirmed by Warell and Blewett (2004). In contrast, Chase et al. (1976) interpreted active and passive microwave data and concluded that Mercury's surface might have a combined $\mathrm{FeO}+\mathrm{TiO}_{2}$ abundance of $\sim 20 \mathrm{wt} \%$, though these workers did not consider other microwave absorbers that could be important (Elachi 1987). Later work by Jeanloz et al. (1995) constrained the $\mathrm{FeO}+\mathrm{TiO}_{2}$ content to $<6 \mathrm{wt} \%$ from microwave opacity measurements. From spectral mixing modeling of mid-infrared measurements, Sprague et al. (2009) found model solutions with rutile $\left(\mathrm{TiO}_{2}\right)$ at abundances as high as $37 \mathrm{wt} \%$ in some areas. Modeling of MASCS spectra by Warell et al. (2010) with Hapke's equations produced solutions with $25 \mathrm{wt} \% \mathrm{TiO}_{2}$, not necessarily in the pure oxide form. This value is much higher than the estimates of Warell and Blewett (2004) derived from modeling of ground-based NIR observations, for which $\mathrm{TiO}_{2}$ was assumed to be in the form of ilmenite. Differences in inferred Ti content among spectral modeling studies may be ascribable in part to the limited mineral libraries used in the modeling. Moreover, spectra from these libraries 

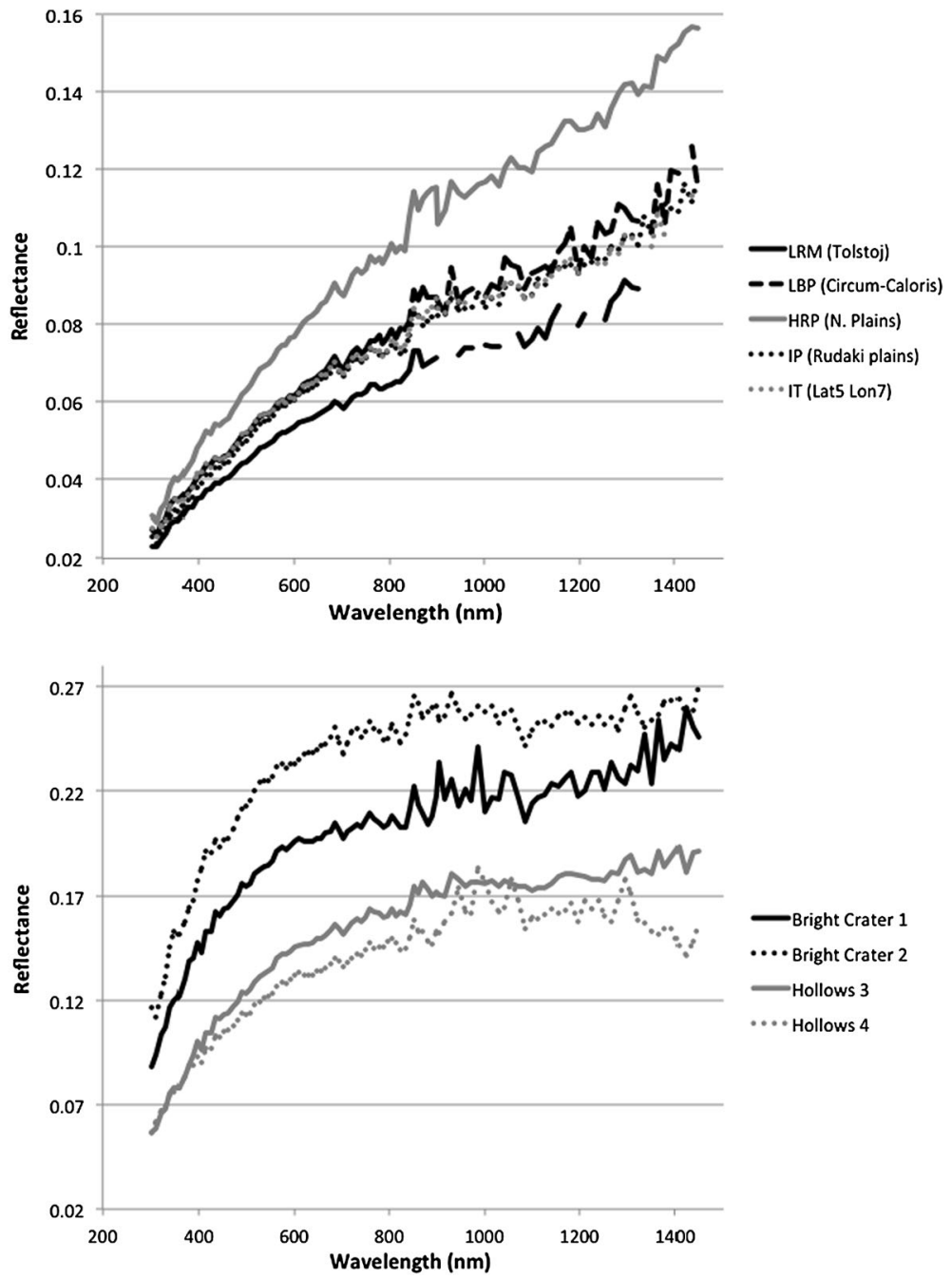

Fig. 24 MASCS spectra of example geologic units, photometrically corrected to $45^{\circ}$ incidence angle, $45^{\circ}$ emission angle, and $90^{\circ}$ phase angle (Izenberg et al. 2014). The top graph contains representative spectra of older geological units that have been exposed to the space-weathering environment for a large fraction of Mercury's geological history. The bottom graph contains representative spectra of younger, less space-weathered units. The spectra in both graphs lack the diagnostic 1- $\mu \mathrm{m}$ feature of FeO in silicates, but the spectra of the younger units display the UV downturn ascribed to OMCT

have typically been acquired at room conditions and do not reflect either the temperature regimes at Mercury or space weathering effects.

From an analysis of Mariner 10 reflectance data, Denevi and Robinson (2008) suggested that Mercury's surface has a spatially variable contribution from a spectrally neutral opaque phase consistent with, but not requiring, Fe-Ti oxides such as ilmenite or ulvöspinel $\left(\mathrm{Fe}_{2} \mathrm{TiO}_{3}\right)$, an inference repeated after early MESSENGER observations of surface color and reflectance (Robinson et al. 2008; Denevi et al. 2009). With spectral mixing models, Denevi et al. (2009) showed that Mercury's spectrally intermediate terrain may have up to 15 vol\% 
ilmenite-equivalent abundance and that the LRM color unit may have up to $40 \mathrm{vol} \%$. Spectral mixing models of mid-infrared spectra of radar-bright region " $C$ " (Harmon et al. 2007) show good model matches with compositions that include perovskite $\left(\mathrm{CaTiO}_{3}\right)(\mathrm{Sprague}$ et al. 2009). Abundant perovskite would indicate extreme enrichment in refractory elements and may also be associated with a large abundance of feldspar ( $\mathrm{KAlSi}_{3} \mathrm{O}_{8}-\mathrm{NaAlSi}_{3} \mathrm{O}_{8}-$ $\mathrm{CaAl}_{2} \mathrm{Si}_{2} \mathrm{O}_{8}$ ).

The Neutron Spectrometer component of MESSENGER's GRNS instrument has also been used to infer the combined abundances of Fe and Ti on Mercury's surface. The NS measures neutrons created by cosmic-ray-spallation reactions in the top meter of Mercury's crust. Thermal neutrons, the down-scattered products of the initial fast neutrons, are highly sensitive to neutron-absorbing elements, of which $\mathrm{Fe}$ and $\mathrm{Ti}$ are typically the most abundant in planetary materials. During MESSENGER's three Mercury flybys, NS measured thermal neutrons across broad areas centered near the equator and measured a total neutron absorption of (45-81) $\times 10^{-4} \mathrm{~cm}^{2} / \mathrm{g}$ (Lawrence et al. 2010). If all of the neutron absorption were due to $\mathrm{Fe}$ and $\mathrm{Ti}$, this figure is equivalent to $\sim 7-18 \mathrm{wt} \%$ ilmenite, broadly consistent with the inference of Denevi et al. (2009) for the fraction of spectrally neutral material in terrain spectrally similar to that sampled by the NS. Alternatively, the neutron absorption could be caused by $8-22 \mathrm{wt} \% \mathrm{Fe}$ or 3-8 wt\% Ti, or some intermediate composition (Lawrence et al. 2010). Moreover, low or even trace quantities of other strongly neutron-absorbing elements (e.g., S, Cl, Gd, Sm) could also contribute to the measured absorption.

Orbital XRS and GRS measurements currently provide the most robust constraints on the surface abundances of Fe and Ti on Mercury. Over a broad set of regions observed by XRS during energetic solar flares, the surface concentration of Fe in any form is inferred to be $\sim 4 \mathrm{wt} \%$ or less (Nittler et al. 2011). The GRS determination of the Fe/Si ratio $(0.077 \pm 0.013$ weighted average) from orbital measurements is within the range reported by XRS and within the errors of the Fe/Si estimates $(0.27 \pm 0.14)$ from flyby GRS observations (Rhodes et al. 2011), suggesting that there is no systematic variation in Fe abundance with latitude (Evans et al. 2012). For a Si abundance of $24.6 \mathrm{wt} \%$, Evans et al. (2012) estimated an Fe abundance, averaged over the northern hemisphere, of $1.9 \pm 0.3 \mathrm{wt} \%$ from the orbital GRS observations.

XRS orbital measurements also yield an upper limit of $\sim 0.8 \mathrm{wt} \% \mathrm{Ti}$ for the same areas for which the Fe abundance has been determined to date (Nittler et al. 2011), indicating that Ti oxides make up at most a minor component of the regolith. XRS has also measured upper limits on the average concentrations of $\mathrm{Mn}(\sim 0.5 \mathrm{wt} \%)$ and $\mathrm{Cl}(\sim 0.2 \mathrm{wt} \%)$, both of which are strong neutron absorbers not considered in the initial NS analysis (Lawrence et al. 2010). The observed abundances or abundance limits on $\mathrm{S}, \mathrm{Fe}, \mathrm{Cl}, \mathrm{Ti}$, and $\mathrm{Mn}$ determined from XRS data are considered generally consistent with the NS flyby measurements (Nittler et al. 2011).

A summary of all observations concerning Fe and Ti in Mercury surface materials is given in Table 8. The inferences from NS and GRS contrast with estimates obtained from modeling of infrared reflectance and emission spectra, although the latter are from data highly affected by space weathering processes not quantitatively considered in the modeling. This contrast between the NS, GRS, and XRS measurements and the results from analyses of reflectance and emission spectra has been used to argue that the Fe on Mercury's surface is not bound in silicates but is contained in other minerals, such as sulfides or metals (Klima et al. 2013; Weider et al. 2013).

Alternatively, much of the Fe and Ti within Mercury's regolith could be exogenic, i.e., delivered by meteoroid and micrometeoroid bombardment. From percentages of meteoritic material within lunar samples, coupled with differences in meteoroid flux between the Moon 
Table 8 Estimates of surface iron and titanium abundances on Mercury ${ }^{\mathrm{a}}$

\begin{tabular}{|c|c|c|c|c|}
\hline Reference & Measurement type & $\begin{array}{l}\mathrm{FeO} \\
(\mathrm{wt} \%)\end{array}$ & $\begin{array}{l}\mathrm{TiO}_{2} \\
(\mathrm{wt} \%)\end{array}$ & $\begin{array}{l}\mathrm{FeO}+\mathrm{TiO}_{2} \\
(\mathrm{wt} \%)\end{array}$ \\
\hline $\begin{array}{l}\text { McCord and Adams (1972a, 1972b); } \\
\text { McCord and Clark (1979 }\end{array}$ & $1 \mu \mathrm{m}$ absorption & $\leq 6$ & & \\
\hline Chase et al. (1976) & Microwave loss tangent & & & $\sim 20 \%$ \\
\hline Hapke (1977) & $1 \mu \mathrm{m}$ absorption & $\sim 3-6$ & & \\
\hline Blewett et al. (1997) & $1 \mu \mathrm{m}$ absorption & $\sim 3$ & $<\sim 1$ & $\sim 4$ \\
\hline Warell and Blewett (2004) & $\begin{array}{l}1 \mu \mathrm{m} \text { absorption, Hapke } \\
\text { modeling }\end{array}$ & $<2$ & $<1$ & \\
\hline Warell et al. (2006) & $1 \mu \mathrm{m}$ absorption & $>\sim 5$ & & \\
\hline McClintock et al. (2008) & $1 \mu \mathrm{m}$ absorption & $<2-3$ & & \\
\hline Sprague et al. (2009) & Mid-IR modeling & $\sim 2-5$ & $<24$ & \\
\hline Warell et al. (2010) & Hapke modeling & $1-8$ & $<25$ & \\
\hline Izenberg et al. (2012) & $\begin{array}{l}1 \mu \mathrm{m} \text { absorption, near-UV } \\
\text { feature }\end{array}$ & $2-4$ & & \\
\hline Klima et al. (2013) & $\begin{array}{l}1 \mu \mathrm{m} \text { absorption, near-UV } \\
\text { feature }\end{array}$ & $<1$ & & \\
\hline Jeanloz et al. (1995) & Microwave loss tangent & & & $<6$ \\
\hline Lawrence et al. (2010) & $\begin{array}{l}\text { Neutron absorption } \\
\text { (flyby) }\end{array}$ & $8-22$ & $3-8$ & 19 \\
\hline Rhodes et al. (2011) & $\begin{array}{l}\text { Gamma-ray emission } \\
\text { (flyby) }\end{array}$ & 9.7 & 7.0 & \\
\hline Evans et al. (2012) & $\begin{array}{l}\text { Gamma-ray emission } \\
\text { (orbital) }\end{array}$ & 1.9 & & \\
\hline Nittler et al. (2011) & $\begin{array}{l}\text { X-ray spectrometry } \\
\text { (orbital) }\end{array}$ & $\leq 4$ & $\leq 0.8$ & \\
\hline
\end{tabular}

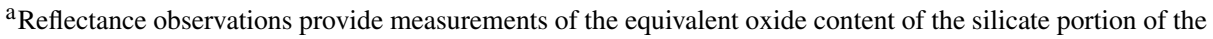
uppermost optical surface of the regolith, whereas microwave, Neutron Spectrometer, and Gamma-Ray Spectrometer observations (Jeanloz et al. 1995; Lawrence et al. 2010; Rhodes et al. 2011) provide measurements of the bulk properties of the regolith (microwave observations penetrate 2 to $20 \mathrm{~cm}$ depth whereas neutron and gamma-ray observations sample the uppermost tens of centimeters to $1 \mathrm{~m}$, depending on composition). XRS samples to depths of $<100 \mu \mathrm{m}$
}

and Mercury (Cintala 1992), Noble et al. (2007) argued that Mercury's regolith could contain 1-5 wt $\%$ exogenous FeO. This range overlaps estimates of Fe abundances determined by the MESSENGER XRS and GRS.

\subsubsection{Metals (Nanophase Iron)}

Mercury's regolith is likely to have $\mathrm{Fe}$ in the form of $\mathrm{npFe}^{0}$ produced by space weathering processes. As discussed above, the $\mathrm{Fe}$ and $\mathrm{Ti}$ within the regolith could be predominantly of exogenic origin. Experiments by Noble et al. (2001) show that concentrations as low as $0.05 \mathrm{wt} \% \mathrm{npFe}^{0}$ are sufficient to influence surface reflectance properties. Hapke (2001) estimated $\sim 0.5 \mathrm{wt} \%$ of $\mathrm{npFe}^{0}$ within Mercury's regolith, similar to that of the Moon, on the basis of theoretical modeling and comparisons with ground-based spectra. Re-analysis by Warell (2003) yielded an upper bound to the $\mathrm{nFFe}^{0}$ abundance of $0.3 \mathrm{wt} \%$, later revised to 0.1-0.2 wt\% with Hapke modeling (Warell and Blewett 2004; 
Table 9 Spectral effects of intermediate-size $n p F e^{0}$ (Noble et al. 2007)

\begin{tabular}{|c|c|c|c|}
\hline $\begin{array}{l}\text { Size range } \\
(\mathrm{nm})\end{array}$ & $\begin{array}{l}\text { Average size } \\
(\mathrm{nm})\end{array}$ & $\begin{array}{l}\text { Concentration } \\
(\mathrm{wt} \%)\end{array}$ & Spectral effect \\
\hline \multirow[t]{4}{*}{$5-15$} & \multirow[t]{4}{*}{8} & 0.02 & $\begin{array}{l}\text { Curvature at visible wavelengths, } \\
\lambda>700 \mathrm{~nm} \text { unaffected }\end{array}$ \\
\hline & & 0.1 & $\begin{array}{l}\text { Curvature at visible wavelengths, } \\
\lambda>700 \mathrm{~nm} \text { unaffected }\end{array}$ \\
\hline & & 0.2 & $\begin{array}{l}\text { Curvature at visible wavelengths, } \\
\lambda>700 \mathrm{~nm} \text { unaffected }\end{array}$ \\
\hline & & $>1$ & Reddening across all wavelengths \\
\hline \multirow[t]{4}{*}{$10-25$} & \multirow[t]{4}{*}{15} & 0.02 & $\begin{array}{l}\text { Steep curvature develops at visible } \\
\text { wavelengths, } \lambda>750 \mathrm{~nm} \text { unaffected }\end{array}$ \\
\hline & & 0.1 & $\begin{array}{l}\text { Spectra redden; curvature becomes less } \\
\text { extreme and extends to longer wavelengths }\end{array}$ \\
\hline & & 0.2 & $\begin{array}{l}\text { Spectra lose curvature and become } \\
\text { increasingly linear }\end{array}$ \\
\hline & & $>1$ & $\begin{array}{l}\text { Spectra become increasingly dark with } \\
\text { convex shape }\end{array}$ \\
\hline \multirow[t]{3}{*}{$25-50$} & \multirow[t]{3}{*}{35} & $<0.1$ & $\begin{array}{l}\text { Slightly curved in visible and red-sloped in } \\
\text { IR ( } \text { similar to } 10-25 \mathrm{~nm} \text { particle size with } \\
0.1 \text { concentration) }\end{array}$ \\
\hline & & $\sim 0.1-1.0$ & Linear, highly red-sloped spectrum \\
\hline & & $>1$ & Increasingly dark, convex-shaped spectrum \\
\hline \multirow[t]{3}{*}{$20-200$} & \multirow[t]{3}{*}{40} & 0.02 & Nearly linear with slightly red slope \\
\hline & & $0.02-1.89$ & $\begin{array}{l}\text { Progressively lower albedo, slight increase } \\
\text { in red slope for } \lambda>1000 \mathrm{~nm}\end{array}$ \\
\hline & & 1.89 & Convex continuum \\
\hline
\end{tabular}

Note: $\lambda$ is wavelength

Warell et al. 2006). Analysis of MASCS spectra has suggested a $\mathrm{npFe}^{0}$ content on Mercury less than that on the Moon (McClintock et al. 2008). Hapke models of MASCS spectra from MESSENGER's first Mercury flyby indicate an $n \mathrm{pFe}^{0}$ abundance of $\sim 0.065 \mathrm{wt} \%$ with small variations between mature and immature regions (Warell et al. 2010). All these estimates for $\mathrm{nFFe}^{0}$ are model-dependent and based on spectra with minimal or no diagnostic absorption features; they were derived from attempts to match the albedo and spectral slope but required assumptions on mineral constituents.

Spectral effects of $\mathrm{nFFe}^{0}$ concentration and particle size have been quantified by laboratory measurements (Noble et al. 2007). Small $\left(<10 \mathrm{~nm}\right.$ in diameter) $\mathrm{npFe}^{0}$ particles markedly redden visible spectra but have little effect at near-infrared wavelengths (Noble et al. 2007), whereas $\mathrm{nFe}^{0}$ particles $>40 \mathrm{~nm}$ in diameter lower albedo across the visibleinfrared portion of the spectrum but do not affect the continuum shape (i.e., no spectral reddening is detected). Spectral changes induced by intermediate-sized particles depend on concentration (Table 9). Studies of mixed sizes show behavior as expected for the average size, biased slightly toward large particle effects. The laboratory measurements on which these conclusions were based were made with transparent silicate gels impregnated with $n p \mathrm{Fe}^{0}$ particles; spectral effects of $\mathrm{npFe}^{0}$ in a more opaque mineral matrix, such as oxides, 
have not been quantified experimentally. The spectral effects of non-pure iron nanoscale particles, such as the $\mathrm{np}(\mathrm{Fe}, \mathrm{Mg}) \mathrm{S}$ observed in the Itokawa samples (Noguchi et al. 2011, 2014), have not yet been studied or quantified in the laboratory.

Coupling Mie theory with the Hapke (2001) radiative transfer equations, Lucey and Riner (2011) modeled spectral effects of $n \mathrm{pFe}^{0}$ particles of different sizes. They predicted that $\mathrm{npFe}{ }^{0}$ particles $<50 \mathrm{~nm}$ in diameter redden spectral slopes, whereas particles larger than $50 \mathrm{~nm}$ cause darkening without reddening, consistent with laboratory spectral studies of lunar analog materials by Noble et al. (2007). Applying their model to near-infrared MASCS spectra of Mercury, Lucey and Riner (2011) suggested that $\mathrm{npFe}^{0}$ is present at $3.5 \mathrm{wt} \%$ in Mercury's regolith, with $3 \mathrm{wt} \%$ in particles larger than $50 \mathrm{~nm}$. Lucey and Riner's (2011) modeling, with variable opaque abundances, showed that spectral darkening effects of $>50$ nm-diameter $\mathrm{npFe}^{0}$ dominate over the darkening effects of opaque minerals; their model matched observations with opaque concentrations of 0 to $24 \mathrm{wt} \%$, demonstrating that opaque minerals are not required to explain Mercury's dark surface.

Noble et al. (2007) concluded that even at small concentrations the size of $n p \mathrm{Fe}^{0}$ particles influences reflectance properties. Lucey and Riner (2011) concluded that all the darkening on Mercury could be entirely, although is not necessarily, produced by large-grain $n p F e{ }^{0}$. Lunar soil studies show that $\mathrm{nFFe}^{0}$ particles in agglutinates are at least twice as large as those in grain rims, $\sim 7 \mathrm{~nm}$ and $\sim 3 \mathrm{~nm}$ in diameter, respectively (Keller and Clemett 2001; Noble et al. 2007). In some lunar agglutinates such $\mathrm{npFe}^{0}$ particles may approach $100 \mathrm{~nm}$ in diameter (Taylor et al. 2001a, 2001b; Noble et al. 2007). Nanophase Fe particles may be large within agglutinates because regolith grains with $\mathrm{npFe}{ }^{0}$-bearing rims are melted to form the agglutinates and the $\mathrm{npFe} \mathrm{e}^{0}$ coalesces to form larger particles (Taylor et al. 2001a, 2001b; Noble et al. 2007). On Mercury, where impacts produce substantially more melt and vapor than on the Moon, and where we expect more agglutinates and vapor-deposited rims, it is plausible that $\mathrm{nFFe}^{0}$ particles will be larger (cf. Lucey and Riner 2011). The formation, melting, and re-formation of agglutinates from prior agglutinates create an environment for the growth of $n \mathrm{pFe}^{0}$ particles.

Another mechanism that has been postulated to coalesce and grow $n p \mathrm{Fe}^{0}$ particles on Mercury is Ostwald ripening (Noble and Pieters 2003). Ostwald ripening occurs in binary mixtures (in this case mineral and $\mathrm{npFe}^{0}$ particles) in which the minority component $\left(\mathrm{npFe}^{0}\right)$ condenses into droplets. Over time the droplets grow in size and their number density decreases. These changes occur because large droplets grow by the condensation of material diffused through the mineral matrix from smaller evaporating droplets (Yao et al. 1993). It has been suggested that Mercury's high dayside temperatures could enlarge $\mathrm{nFFe}^{0}$ particles, especially in the hottest areas (low latitudes and the "hot pole" longitudes), where spectral differences would be expected (Noble et al. 2007). Contrasting with finer $\mathrm{nFe}^{0}$ grain sizes at cooler high latitudes and associated reddened spectral continua, larger average $n \mathrm{pF}^{0}$ particles near the equator might result in a flatter, darker spectrum. MESSENGER multispectral images (Robinson et al. 2008; Blewett et al. 2009; Denevi et al. 2009) have not yet revealed systematic latitudinal color contrasts consistent with Ostwald ripening, but interpretation is complicated by color variations associated with compositional units and by high-reflectance crater rays. However, Ostwald ripening usually refers to emulsions or magmatic systems (i.e., systems with high atomic mobility in which diffusion can be driven by surface energy gradients). The melting temperature of agglutinates is expected to be 2 to 3 times Mercury's maximum surface temperature, so the diffusive timescales may be too short to drive Ostwald ripening, even on geologic timescales. Regardless of the growth mechanism, Starukhina and Shkuratov (2003) noted that continued growth of iron grains to sizes $\sim 0.1$ to $1.0 \mu \mathrm{m}$ would cause a decrease in absorption and an increase in scattering. This effect might make "overmatured" soils brighter. 
Noble et al. (2007) showed that the combination of small $n p F e^{0}$ particles in rims with larger $\mathrm{nFFe}^{0}$ particles in agglutinates accounts for the shape of lunar soil spectra. On the basis of telescopic spectra of Mercury, they predicted that Mercury's regolith has more and larger $\mathrm{nFe}^{0}$ grains than the Moon, whereas S-class asteroid surfaces should have slightly smaller $\mathrm{nFe}^{0}$ particles than the Moon, consistent with the paucity of agglutinates in regolith breccia meteorites (e.g., Basu and McKay 1983), the $\mathrm{npFe}^{0}$ particles observed in the Itokawa samples (Noguchi et al. 2011), and evidence that solar wind irradiation is a key weathering process on asteroids (Loeffler et al. 2009; Vernazza et al. 2009). Recent laboratory analyses of regolith breccia meteorites support these predictions. Noble et al. (2011) found small, though extremely scarce, $\mathrm{npFe}^{0}$ in mineral rims within these meteorites.

Additional supporting evidence for smaller $n \mathrm{pFe}^{0}$ particles on asteroidal surfaces has been obtained from an examination of samples returned from Itokawa (Noguchi et al. 2011). Noguchi et al. (2011) found $\mathrm{nFe}^{0}$ particles with an average grain size of $2 \mathrm{~nm}$ within five of the ten Itokawa grain samples they examined. Noguchi et al. (2011) also found that compositional variations within the nanophase particles correlated with location within the regolith grains. Sulfur-bearing Fe-rich nanoparticles (npFeS) were detected in thin (5-15 nm) surface layers on olivine, low-Ca pyroxenes, and plagioclase (Noguchi et al. 2011). Sulfur-free Fe-rich nanoparticles were found deeper $(<60 \mathrm{~nm})$ inside the rims of ferromagnesium silicates (Noguchi et al. 2011). Noguchi et al. (2011) showed that the structure of the rims on these asteroidal grains indicate that $\mathrm{npFeS}$ particles near the surface form via vapor deposition, and the $\mathrm{nFe}^{0}$ particles deeper inside the rims are formed by radiation-induced amorphization and in situ reduction of Fe. More recent analyses of the Itokawa samples also show the presence of a component of $\mathrm{MgS}$ nanophase particles in the vapor-deposited top layer of the sample grains (Noguchi et al. 2014).

These compositional differences have implications for Mercury, especially in light of the detection of high sulfur abundance on Mercury (Nittler et al. 2011). The XRS observations indicate sulfur abundances ranging from $\sim 1$ up to $4 \mathrm{wt} \%$, in contrast to an estimate of $\leq 0.2 \mathrm{wt} \%$ in bulk silicate Earth, lunar silicates, stony meteorites from Mars, and differentiated asteroids (Nittler et al. 2011). GRS orbital data have confirmed these results, indicating an average northern hemisphere $\mathrm{S}$ abundance of $2.3 \pm 0.4 \mathrm{wt} \%$ (Evans et al. 2012). If $n p F e S$ particles are associated with vapor deposition, and the production of vapor on Mercury is higher than on both the Moon and asteroids, this composition of nanoparticle can be expected within a surface containing 1-4 wt\% of both Fe and S. It also offers opportunity for speculation on nanophase particles of compositions other than Fe, such as $\mathrm{Mg}$, which often substitutes for Fe in the lattice matrix of many minerals and could be created through more intense reduction processes. The XRS and GRS orbital measurements indicating Mg-rich mafic silicates (Evans et al. 2012), and the detection of $n p(\mathrm{Fe}, \mathrm{Mg}) \mathrm{S}$ particles in vapor-deposited coatings on the Itokawa grain samples (Noguchi et al. 2014), are in line with this suggestion.

\subsubsection{Silicates (Plagioclase, Pyroxenes, Olivines)}

Silicates, such as plagioclase feldspar, pyroxene, and olivine, are indicated for Mercury's surface from emission and reflectance spectra (Sprague et al. 1994, 2002, 2007, 2009; Emery et al. 1998; Warell and Blewett 2004; Warell et al. 2006). Feldspar emissions in the mid-infrared were reported (Emery et al. 1998), and 14-28 wt\% Na- and K-bearing feldspars were estimated for different locations from comparisons with models involving combinations of laboratory spectra (Sprague et al. 2009). Ground-based mid-infrared telescopic data have been interpreted to indicate magnesium-bearing orthopyroxenes, calciumbearing clinopyroxenes, sodium-bearing plagioclase, and minor amounts of magnesian 
Table 10 Inferences on silicate minerals present on Mercury

\begin{tabular}{|c|c|c|c|c|c|}
\hline Reference & Data source & Plagioclase & Pyroxene & Olivine & Sulfides \\
\hline $\begin{array}{l}\text { Sprague et al. } \\
\text { (1994) }\end{array}$ & $\begin{array}{l}\text { Emission } \\
\text { spectra }\end{array}$ & $\begin{array}{l}\text { Na-bearing } \\
\text { feldspar }\end{array}$ & Enstatite & & \\
\hline $\begin{array}{l}\text { Sprague et al. } \\
\text { (1995) }\end{array}$ & $\begin{array}{l}\text { Emission } \\
\text { spectra }\end{array}$ & & & & $\begin{array}{l}\text { Oldhamite } \\
(\mathrm{Ca}, \mathrm{Mg}, \mathrm{Fe}) \mathrm{S}\end{array}$ \\
\hline $\begin{array}{l}\text { Emery et al. } \\
\text { (1998) }\end{array}$ & $\begin{array}{l}\text { Emission } \\
\text { spectra }\end{array}$ & $\begin{array}{l}\text { Feldspar and } \\
\text { feldspathoids }\end{array}$ & & $\begin{array}{l}\text { Mg-rich } \\
\text { indicated }\end{array}$ & \\
\hline $\begin{array}{l}\text { Warell et al. } \\
(2006)\end{array}$ & $\begin{array}{l}\text { Reflectance } \\
\text { spectra }\end{array}$ & & $\begin{array}{l}\text { Ca-rich } \\
\text { clinopyroxene }\end{array}$ & & \\
\hline $\begin{array}{l}\text { Sprague et al. } \\
(2002)\end{array}$ & & & $\begin{array}{l}\text { Diopside } \\
\left(\mathrm{MgCaSi}_{2} \mathrm{O}_{6}\right)\end{array}$ & & \\
\hline $\begin{array}{l}\text { Sprague et al. } \\
(2009)\end{array}$ & $\begin{array}{l}\text { Mid-IR } \\
\text { modeling }\end{array}$ & $\begin{array}{l}\text { K-feldspar within } \\
\text { Caloris basin, } \\
\text { Na-bearing } \\
\text { plagioclase at } \\
\text { various locations }\end{array}$ & $\begin{array}{l}\text { Ca-rich } \\
\text { clinopyroxene, } \\
\text { hedenbergite, } \\
\text { Mg-rich } \\
\text { orthopyroxene }\end{array}$ & & \\
\hline $\begin{array}{l}\text { Warell et al. } \\
(2010)\end{array}$ & $\begin{array}{l}\text { Hapke } \\
\text { models of } \\
\text { reflectance } \\
\text { spectra }\end{array}$ & $\begin{array}{l}\text { 35-70 mode\% } \\
\text { Na-plag OR } \\
\text { orthoclase } \\
\text { (K-fledspar) } \\
\text { Up to } 70 \text { mode\% } \\
\text { pyroxene- and } \\
\text { ilmenite-bearing } \\
\text { glasses }\end{array}$ & $\begin{array}{l}\leq 30 \text { mode } \% \\
\text { Mg-rich } \\
\text { clinopyroxene } \\
<5 \text { mode } \% \\
\text { Mg-rich } \\
\text { orthopyroxene } \\
<21 \text { wt } \% \text { MnO } \\
\text { (hedenbergite) }\end{array}$ & $\begin{array}{l}\text { Minute } \\
\text { amounts in } \\
\text { the glass } \\
\text { samples used } \\
\text { in the model }\end{array}$ & \\
\hline $\begin{array}{l}\text { Nittler et al. } \\
(2011)\end{array}$ & $\begin{array}{l}\text { Orbital } \\
\text { X-ray } \\
\text { spectra }\end{array}$ & $\begin{array}{l}\text { Depletion of } \\
\text { plagioclase } \\
\text { relative to the } \\
\text { lunar crust }\end{array}$ & & & \\
\hline $\begin{array}{l}\text { Weider et al. } \\
(2012)\end{array}$ & $\begin{array}{l}\text { Orbital } \\
\text { X-ray and } \\
\text { gamma-ray } \\
\text { spectra }\end{array}$ & $\begin{array}{l}\text { Ca-bearing, } \\
\text { Na-bearing, and } \\
\text { K-rich feldspars, } \\
\text { but depletion of } \\
\text { plagioclase } \\
\text { relative to the } \\
\text { lunar crust }\end{array}$ & $\begin{array}{l}\text { Mg-rich } \\
\text { (Enstatite) }\end{array}$ & $\begin{array}{l}\text { Mg-rich } \\
\text { (Forsterite) }\end{array}$ & $\begin{array}{l}\text { Oldhamite } \\
(\mathrm{Ca}, \mathrm{Mg}, \mathrm{Fe}) \mathrm{S} \\
\text { Possible } \\
\text { presence of } \\
\text { niningerite } \\
(\mathrm{MgS}) \\
\text { and/or } \\
\text { daubreelite } \\
\left(\mathrm{FeCr}_{2} \mathrm{~S}\right)\end{array}$ \\
\hline
\end{tabular}

olivine (Sprague et al. 2002, 2009). Olivine has been indicated from a comparison of midinfrared emission features with laboratory spectra of Mg-rich olivines (Emery et al. 1998; Sprague et al. 2009). Telescopic observations at high northern and southern latitudes (Warell et al. 2006) have been interpreted to imply localized low-Fe, high-Ca pyroxenes. Exospheric identifications of Na, K (Potter and Morgan 1985, 1986; Sprague et al. 1989), and Mg (McClintock et al. 2009) support the presence of minerals bearing these cations. Although surface concentrations have not been quantified from exospheric measurements, the elemental abundances of these cations have been measured by the MESSENGER XRS (Mg) and GRS (Na and K) (Peplowski et al. 2012; Starr et al. 2012; Weider et al. 2012) and are discussed at the end of this section. Table 10 summarizes the current evidence for various silicate mineral assemblages.

Examples of near-infrared and mid-infrared spectra, upon which these silicate mineral identifications are based, are displayed in Fig. 25. These mineral identifications rely on 
Fig. 25 (Top) Visible to NIR spectra acquired with the Infrared Telescope Facility for three different locations on Mercury (gray) compared with other ground-based observations (black). Each of these spectra has solar reflectance and thermal emission components removed, is divided by a linear fit to the continuum, and is normalized at $1 \mu \mathrm{m}$. Two of these three spectra (2003 S and $2003 \mathrm{~N}$ ) display evidence for an absorption band between 0.8 and $1.1 \mu \mathrm{m}$ associated with $\mathrm{FeO}$ in silicates. These observations are from Warell et al. (2006). (Bottom) Comparison of a mid-infrared spectrum (Sprague et al. 2009) with spectra of laboratory samples. Peaks associated with pyroxene and labradorite plagioclase feldspar are seen near 5 and $8 \mu \mathrm{m}$, respectively (Strom and Sprague 2003)
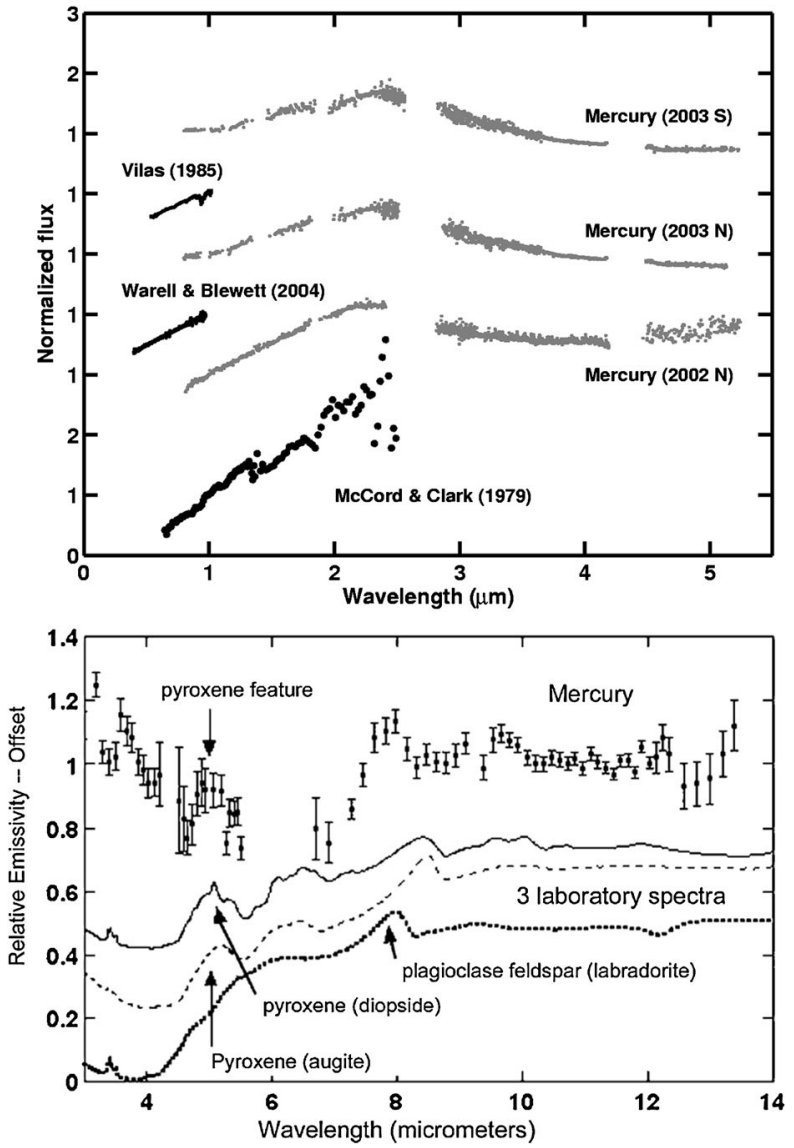

comparisons with spectral measurements of minerals generally taken at room temperature, under standard photometric geometries (incidence, emission, and phase angle values of $0^{\circ}$, $30^{\circ}, 30^{\circ}$, respectively), and without exposure to space weathering conditions. These conditions do not match those of Mercury's surface or surface observation geometries. The mineral quantities derived from the mid-infrared modeling also would produce detectable features in the NIR, which are not observed. Whereas this discrepancy could be attributed to the differences between laboratory conditions and those at Mercury's surface, this attribution has not been proven. Studies of the effects of temperature, photometry, and weathering at both mid- and near-infrared wavelengths are required to resolve this issue.

MESSENGER's XRS instrument has provided orbital measurements of $\mathrm{Mg} / \mathrm{Si}, \mathrm{Al} / \mathrm{Si}$, $\mathrm{S} / \mathrm{Si}$, and $\mathrm{Ca} / \mathrm{Si}$ ratios by weight and found that surface material on Mercury is higher in $\mathrm{Mg} / \mathrm{Si}$ and $\mathrm{S} / \mathrm{Si}$ and lower in $\mathrm{Al} / \mathrm{Si}$ and $\mathrm{Ca} / \mathrm{Si}$ than terrestrial and lunar crustal material, indicative of lower plagioclase feldspar abundances (Nittler et al. 2011). Nittler et al. (2011) argued that the relatively low $\mathrm{Al} / \mathrm{Si}$ and $\mathrm{Ca} / \mathrm{Si}$ ratios of Mercury surface materials are evidence against a plagioclase-rich crust similar to the crust that formed the lunar highlands by flotation of solidifying feldspar during cooling and crystallization of a global magma ocean. Depending on the Fe content of the bulk silicate fraction of Mercury, however, plagioclase crystals may not float in an early magma ocean on Mercury, in contrast to the situation on the Moon (Brown and Elkins-Tanton 2009). Although the role of a global magma ocean in 
the formation of Mercury's early crust is not known, the higher $\mathrm{Mg} / \mathrm{Si}$ ratio and low $\mathrm{Fe} / \mathrm{Si}$ ratio of surface material is indicative that $\mathrm{Mg}$-rich silicates, such as enstatite and forsterite, dominate the surface mineral chemistry (Peplowski et al. 2014).

$\mathrm{X}$-ray fluorescence (XRF) observations from solar flares on the dayside (Weider et al. 2012) and from electron-induced X-ray fluorescence (eXRF) on the nightside (Starr et al. 2012) show spatial variability in $\mathrm{Mg}, \mathrm{Al}, \mathrm{S}$, and $\mathrm{Ca}$ abundances across Mercury's surface. Spatial variation and correlations between elemental abundances for broad unit regions within the northern hemisphere as measured with the XRS by Weider et al. (2012) are displayed in Figs. 26 and 27. The units examined include the northern plains (NP), a subset of the smooth plains defined by Denevi et al. (2009), and intercrater plains and heavily cratered terrain (IcP-HCT), which are older deposits that surround the NP. On average the IcP-HCT display higher $\mathrm{Mg} / \mathrm{Si}, \mathrm{S} / \mathrm{Si}$, and $\mathrm{Ca} / \mathrm{Si}$ ratios and lower $\mathrm{Al} / \mathrm{Si}$ ratios than the $\mathrm{NP}$ (Weider et al. 2012). Even within the older IcP-HCT there appear to be two compositional units, one with $\mathrm{Mg} / \mathrm{Si}$ and $\mathrm{Al} / \mathrm{Si}$ rates of $\sim 0.75$ and $\sim 0.15$, respectively, and another with ratio values of $\sim 0.45$ and $\sim 0.3$, respectively (Weider et al. 2012), as indicated in Fig. 26 . Weider et al. (2012) argue that the elemental compositional variations between the NP and the older IcP-HCT indicates that the younger smooth plains region (NP) may have been derived from a more chemically evolved magma source.

Correlations between $\mathrm{Ca} / \mathrm{Si}$ and $\mathrm{S} / \mathrm{Si}$ ratios are found in all the terrains examined by Weider et al. (2012). This correlation is consistent with the presence of oldhamite, $(\mathrm{Ca}, \mathrm{Mg}, \mathrm{Fe}) \mathrm{S}$ (Weider et al. 2012), which was initially suggested as a surface mineral constituent by Sprague et al. (1995) on the basis of ground-based spectral observations. Figure 26f shows a slight anti-correlation between $\mathrm{Al} / \mathrm{Si}$ and $\mathrm{S} / \mathrm{Si}$, indicating that oldhamite cannot fully account for the $\mathrm{Ca}-\mathrm{S}$ correlation since its presence would not affect the $\mathrm{Al}$ abundance (Weider et al. 2012). Weider et al. (2012) suggest that the $\mathrm{Ca}-\mathrm{S}$ correlation is influenced by a variable abundance of oldhamite and the mixing of varying amounts of enstatite, forsterite, and plagioclase feldspar. Although these XRS measurements are consistent with the idea that much of the $\mathrm{S}$ is present as $\mathrm{CaS}$, the majority of Ca must be in plagioclase feldspar (StockstillCahill et al. 2012; Weider et al. 2012). The $\mathrm{Ca} / \mathrm{Si}$ and $\mathrm{Al} / \mathrm{Si}$ ratios don't correlate as expected if Ca-rich plagioclase (anorthite, $\mathrm{CaAl}_{2} \mathrm{Si}_{2} \mathrm{O}_{8}$ ) were the only $\mathrm{Ca}-\mathrm{Al}$-bearing mineral phase, so it has been postulated that $\mathrm{Ca}$ is present in one or more non-aluminous, non-sulfide mineral phases such as diopside, $\mathrm{MgCaSi}_{2} \mathrm{O}_{6}$ (Weider et al. 2012). Diopside was inferred to be present in an equatorial region ranging from $275^{\circ}$ to $315^{\circ} \mathrm{E}$ by Sprague et al. (2002) on the basis of ground-based observations. Possible correlations between $\mathrm{Mg} / \mathrm{Si}$ and $\mathrm{Ca} / \mathrm{Si}$ in the IcP-HCT regions are suggestive of Ca-rich pyroxene, though this mineral phase would not account for all of the $\mathrm{Al}$ measured in these regions (Weider et al. 2012). The younger NP are richer in Al than the older IcP-HCT, suggesting that the NP contain more feldspar (Weider et al. 2012).

MESSENGER's GRS has measured K abundances across Mercury that also display strong spatial variations in the northern hemisphere (Peplowski et al. 2012). Higher K abundances are measured in regions associated with the NP ( $2000 \mathrm{ppm})$ than for the surrounding units $(\sim 500 \mathrm{ppm})$, though the correlation with surface temperature is at least as strong as that with geologic unit (Peplowski et al. 2012; Weider et al. 2012). A similar enrichment of $\mathrm{Na}$ at high northern latitudes suggests that the NP feldspars may contain K-bearing and Na-bearing variants, as well as calcic feldspar. The anticorrelation between $\mathrm{K}$ and $\mathrm{Na}$ abundances and near-surface temperature (Peplowski et al. 2012), however, suggests that some of the $\mathrm{K}$ and $\mathrm{Na}$ measured may be present as adsorbates within the regolith. Table 11 summarizes the range in elemental abundances measured by both the XRS and GRS. These elemental abundances derived from orbital operations have placed important constraints on possible mineral compositions, which are summarized in Table 10. 


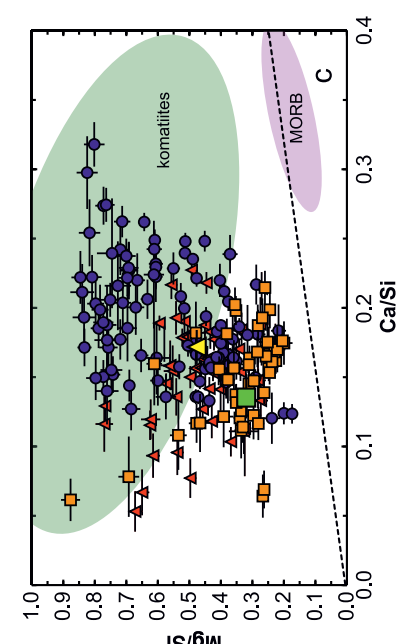

!s/6W
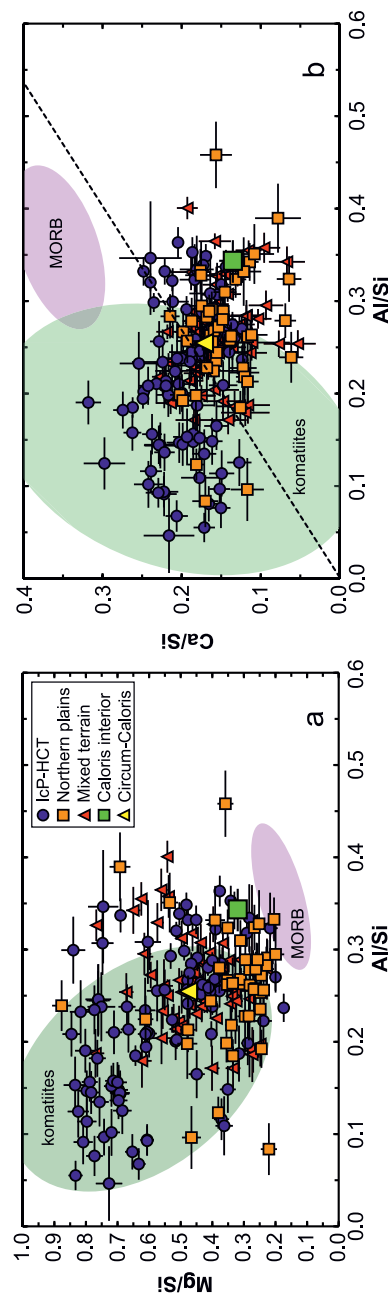
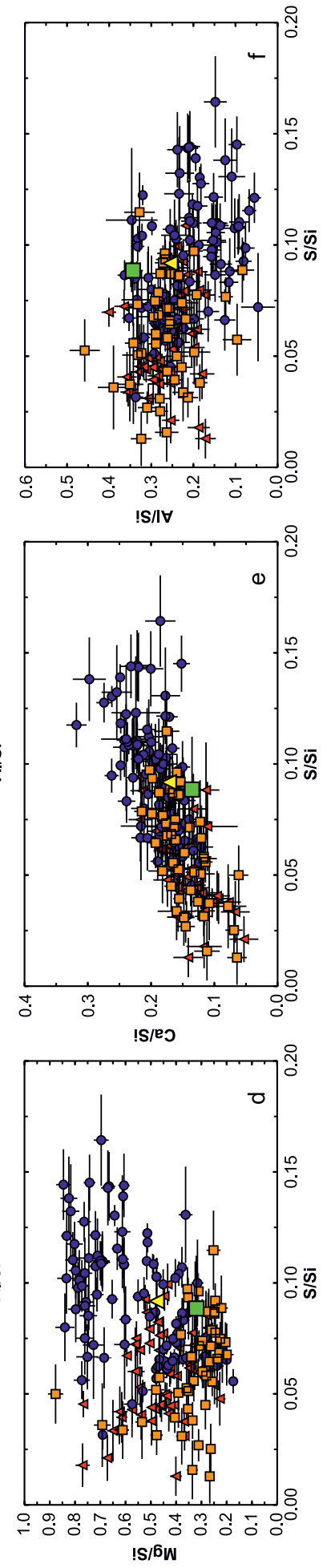

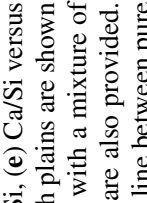

施

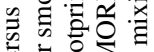

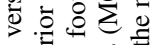

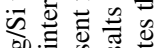

$\sum 0$

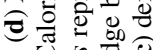

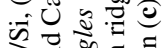

సิ

क ष

क्षे

运范

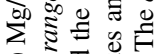

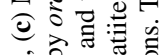

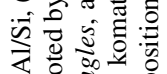

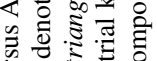

D.

运艺造

का दू

定

. $\frac{0}{4}$

एँ

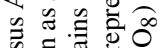

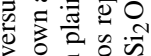

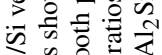

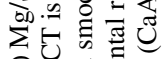

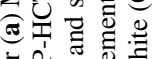

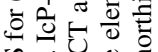

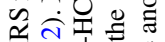

주월

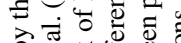

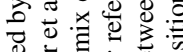

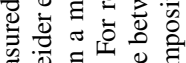

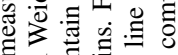

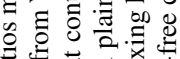

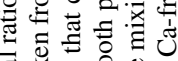

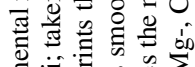

:

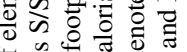

पั0

.

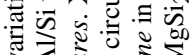

ᄂ

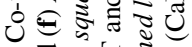

ั

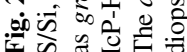


Fig. 27 Histograms of elemental ratios for three terrain types measured by the MESSENGER XRS for (a) $\mathrm{Mg} / \mathrm{Si}$, (b) $\mathrm{Al} / \mathrm{Si}$, (c) $\mathrm{S} / \mathrm{Si}$, and (d) $\mathrm{Ca} / \mathrm{Si}$. The data have been divided into 10 equal sized bins and normalized to the total number of data points for each elemental ratio shown. The bin upper limits are shown on the abscissae. From Weider et al. (2012)
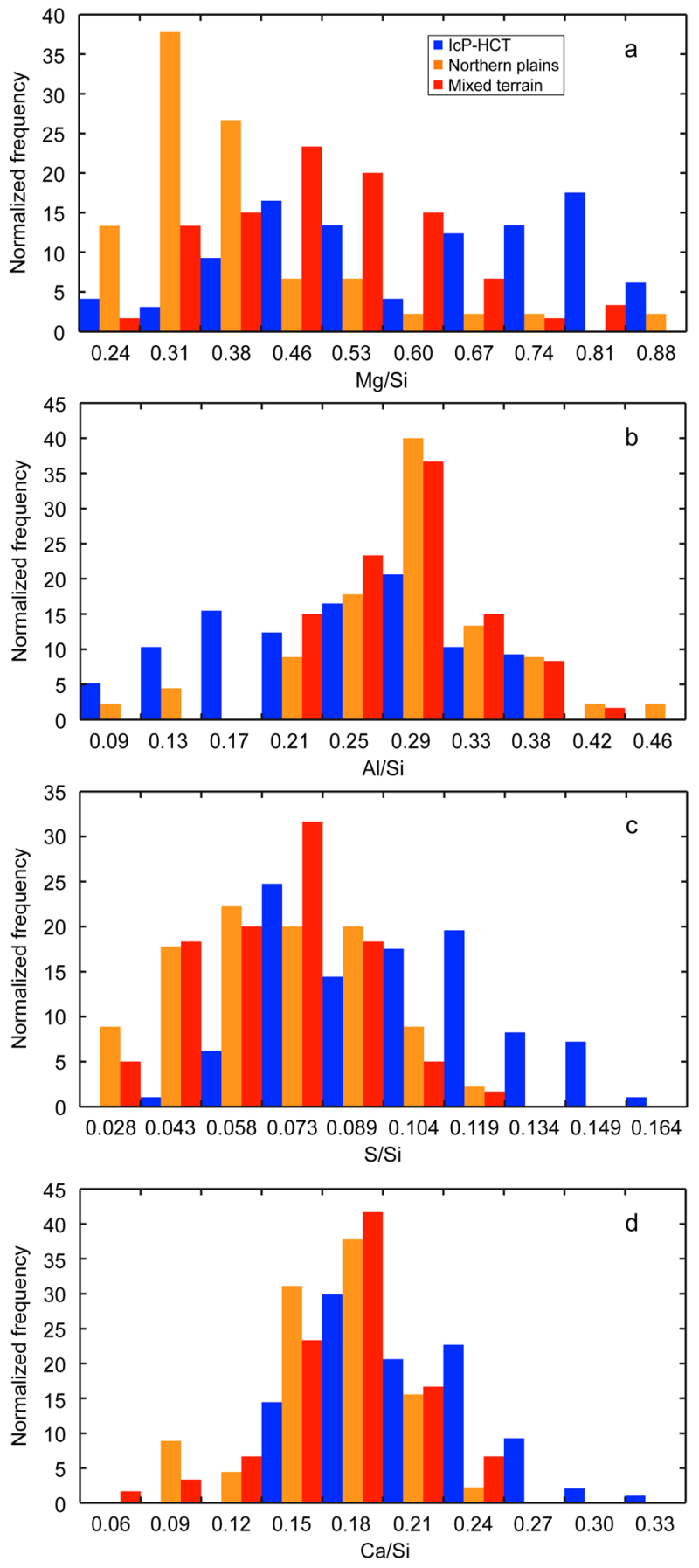
Table 11 Elemental abundance variations across Mercury

\begin{tabular}{lllll}
\hline Element & Global & Northern plains & Caloris basin & IcP-HCT \\
\hline $\mathrm{Mg}$ & $2-21.1 \mathrm{wt}^{\mathrm{a}}$ & $\sim 8.5 \mathrm{wt} \%^{\mathrm{b}}$ & $\sim 8.0 \mathrm{wt} \%^{\mathrm{b}}$ & $\sim 14.4 \mathrm{wt} \%^{\mathrm{b}}$ \\
$\mathrm{Al}$ & $4.3-13.1 \mathrm{wt}^{\mathrm{a}}$ & $\sim 6.6 \mathrm{wt} \%^{\mathrm{b}}$ & $\sim 8.5 \mathrm{wt} \%^{\mathrm{b}}$ & $\sim 5.4 \mathrm{wt} \%^{\mathrm{b}}$ \\
$\mathrm{S}$ & $1.7-7.6 \mathrm{wt}^{\mathrm{a}}$ & $\sim 1.5 \mathrm{wt} \%^{\mathrm{b}}$ & $\sim 2.3 \mathrm{wt} \%^{\mathrm{b}}$ & $\sim 2.3 \mathrm{wt}^{\mathrm{b}}$ \\
$\mathrm{Ca}$ & $3.3-15.1 \mathrm{wt} \%^{\mathrm{a}}$ & $\sim 3.7 \mathrm{wt} \%^{\mathrm{b}}$ & $\sim 3.5 \mathrm{wt} \%^{\mathrm{b}}$ & $\sim 4.9 \mathrm{wt}^{\mathrm{b}}$ \\
$\mathrm{K}$ & $1288 \pm 234 \mathrm{ppm}^{\mathrm{c}}$ & $1786 \pm 330 \mathrm{ppm}^{\mathrm{c}}$ & $754 \pm 203 \mathrm{ppm}^{\mathrm{c}}$ & $952 \pm 176 \mathrm{ppm}^{\mathrm{c}}$ \\
$\mathrm{Th}$ & $0.155 \pm 0.054 \mathrm{ppm}^{\mathrm{c}}$ & $0.142 \pm 0.075 \mathrm{ppm}^{\mathrm{c}}$ & $0.540 \pm 0.288 \mathrm{ppm}^{\mathrm{c}}$ & $0.168 \pm 0.072 \mathrm{ppm}^{\mathrm{c}}$ \\
\hline
\end{tabular}

a Abundances are taken from Starr et al. (2012). The ranges include one standard deviation and are based on a 25.7 wt\% Si abundance. ${ }^{b}$ Abundances are taken from Weider et al. (2012) and are based on a $25 \mathrm{wt} \% \mathrm{Si}$ abundance. ${ }^{\mathrm{c}}$ Abundances are taken from Peplowski et al. (2012)

\subsubsection{Oxides and Opaque Minerals}

As noted above, Mercury's low average albedo suggests that its regolith may contain a spectrally neutral opaque component (Warell et al. 2006; Robinson et al. 2008; Denevi et al. 2009). Low-reflectance iron- and titanium-bearing oxides [such as ilmenite, perovskite, ulvöspinel $\left(\mathrm{Fe}_{2} \mathrm{TiO}_{4}\right)$, armalcolite $\left((\mathrm{Mg}, \mathrm{Fe}) \mathrm{Ti}_{2} \mathrm{O}_{5}\right)$, and ferropseudobrookite $\left.\left(\mathrm{FeTiO}_{5}\right)\right]$ have been suggested as candidates (McClintock et al. 2008; Robinson et al. 2008; Denevi et al. 2009; Sprague et al. 2009). Hapke models of MESSENGER MASCS data suggest the possible presence of lunar-like opaque minerals at $<10 \mathrm{wt} \%$ levels (Warell et al. 2010). Riner et al. (2009) argued that Fe-Ti oxides cannot be the sole darkening agent, because 20$50 \mathrm{wt} \%$ abundances would be required to match measured reflectance properties. For instance, they inferred that the abundance of ilmenite needed to darken immature lunar highland sample material to match Mercury's reflectance at $490 \mathrm{~nm}$ is $27-38 \mathrm{wt} \%$ (18.5-21.9 equivalent wt $\% \mathrm{Fe}+\mathrm{Ti}$ ), at the high end or above the MESSENGER flyby NS and GRS estimates and well above the abundances indicated by the orbital XRS and GRS observations. Alternative opaque minerals include geikielite $\left(\mathrm{MgTiO}_{3}\right)$, the magnesian end-member of a solid-solution series with ilmenite, and anosovite $\left(\mathrm{Ti}_{3} \mathrm{O}_{5}\right)$, which forms a solid-solution series with armalcolite (Riner et al. 2009). Because laboratory reflectance data are lacking for these oxides, Riner et al. (2009) did not estimate abundances for these minerals on Mercury. If such minerals are present, the abundances indicated by the XRS would be low, however, and may be insufficient to produce the darkening observed.

Lucey and Riner (2011) modeled spectra by varying the amounts and sizes of $n p F e^{0}$ and of opaque iron oxide minerals. Comparison with MASCS NIR spectra showed that Mercury's albedo and spectral slope could be matched with 0-24 wt\% opaque minerals. The best spectral matches were for $3 \mathrm{wt} \%$ large $(>50 \mu \mathrm{m}) \mathrm{npFe}^{0}$ particles and $0.5 \mathrm{wt} \%$ small $(<50 \mu \mathrm{m}) \mathrm{npFe}^{0}$ particles, regardless of the opaque content in the model; their total $\mathrm{npFe}^{0}$ content is within the measured XRS abundance limit (Nittler et al. 2011). Lucey and Riner (2011) predicted a 4-6 wt\% opaque mineral content if all $\mathrm{npFe}^{0}$ is from the opaque iron oxides discussed above rather than from silicates.

Modeling by Warell et al. (2010) suggested abundances for other (non Fe- or Ti-bearing) oxides, such as $\mathrm{SiO}_{2}(40-50 \mathrm{wt} \%), \mathrm{Al}_{2} \mathrm{O}_{3}(10-35 \mathrm{wt} \%)$, and $\mathrm{MnO}$ (<21 wt\%). GRS flyby measurements indicated silicon $(\mathrm{Si})$ at a $99 \%$ confidence level, and bounds on other elements were derived from ratioing with an assumed abundance for Si of $18 \mathrm{wt} \%$ (Rhodes et al. 2011). Sprague et al. (2009) estimated $\mathrm{SiO}_{2}$ content from mid-IR spectra as 49 to 
$55 \mathrm{wt} \%$. GRS observations of $\mathrm{Si}$ abundance in the northern hemisphere show no Si abundance variability above the two-standard-deviation level (Peplowski et al. 2012). Table 11 summarizes the XRS and GRS abundances for some of the major mineral-forming elements.

\subsubsection{Darkening Agents}

Here we consider additional darkening agents that might contribute to Mercury's low albedo. Beyond the ferrous iron oxides, impact glasses (including agglutinates), and $\mathrm{npFe}^{0}$ discussed above, sulfides, clinopyroxenes with strongly absorbing cations (such as $\mathrm{Mn}$ and $\mathrm{Cr}$ ), temperature effects, meteoritic material, and both indigenous and exogenic carbon are also possibilities (Helbert and Maturilli 2009; Warell et al. 2010).

Riner et al. (2009) contended that Mercury's low reflectance and apparent low Fe content argue against the formation of Fe-Ti-rich oxides, which would require the co-existing silicates also to be Fe-rich (Riner et al. 2009), which is contra-indicated by the lack of a $1 \mu \mathrm{m}$ band in reflectance spectra and the Fe abundance measured by the XRS. Ilmenite displays a reflectance peak near $1 \mu \mathrm{m}$. Hapke models by Riner et al. (2009) showed that $16-40 \mathrm{wt} \%$ ilmenite would be needed to completely mask the $1 \mu \mathrm{m}$ feature in a lunar highland soil with $5.14 \mathrm{wt} \% \mathrm{FeO}$. More Mg-rich oxides with low-Fe silicates, possibly combined with some fine-grained ilmenite to mask the ferrous iron absorption near $1 \mu \mathrm{m}$, are more geochemically plausible (Riner et al. 2009).

Burbine et al. (2002) demonstrated that the properties of Mercury's crust, as then known, could be described with a mineral assemblage similar to that in aubrite meteorites, which contain such minerals as enstatite $\left(\mathrm{MgSiO}_{3}\right)$, plagioclase $\left(\mathrm{NaAlSi}_{3} \mathrm{O}_{8}-\mathrm{CaAlSi}_{2} \mathrm{O}_{8}\right)$, diopside, and $\mathrm{Ca}, \mathrm{Mg}$, and $\mathrm{Mn}$ sulfides. It has been suggested that $\mathrm{Mg}$ and $\mathrm{Ca}$ sulfides could contribute to Mercury's low reflectance, given the high abundance of sulfur and apparent correlations among S, Mg, and Ca (Nittler et al. 2011; Weider et al. 2012). The presence of oldhamite in both enstatite chondrites and aubrite meteorites indicates that this mineral is stable under highly reducing environments (Ebel 2006), and when taken in conjunction with the XRS elemental abundance measurements supports its presence on Mercury's surface (Weider et al. 2012).

Clinopyroxenes with strongly absorbing cations, such as $\mathrm{Mn}$ and $\mathrm{Cr}$, are additional possible darkening agents (Warell et al. 2010). Because manganese is also a strong neutron absorber, its presence would affect NS estimates of Fe and Ti abundances deduced from the MESSENGER NS data but not from GRS data. Differences among the NS, GRS, and XRS estimates for $\mathrm{Fe}$ and Ti discussed above leave room for other strong neutron absorbers within Mercury's regolith (such as $\mathrm{Sm}, \mathrm{Gd}, \mathrm{Zn}, \mathrm{Mn}$, and $\mathrm{Pb}$ ). However, orbital GRS measurements to date have not detected elements such as $\mathrm{Cl}, \mathrm{Cr}$, or $\mathrm{Mn}$, which have large neutron capture cross-sections. This non-detection may be due to low detector efficiencies at the high energies at which these elements emit gamma rays (Evans et al. 2012). XRS observations, however, have placed upper limits on the average concentrations of $\mathrm{Mn}$ and $\mathrm{Cl}$ to be $\sim 0.5$ and $0.2 \mathrm{wt} \%$, respectively (Nittler et al. 2011).

Given Mercury's proximity to the Sun, thermal effects must also be considered. Ostwald ripening of $\mathrm{npFe}^{0}$ grains, one such effect, was mentioned above. Helbert and Maturilli (2009) examined mid-infrared emission from labradorite feldspar (a possible Mercury ana$\log$ on the basis of pre-MESSENGER ground-based observations, Sprague et al. 2009) at temperatures up to $420{ }^{\circ} \mathrm{C}$, revealing emissivity changes with temperature. Their samples were visibly and measurably darker at Mercury's dayside temperatures, though such changes may have been due to oxidation of trace Fe through exposure to air during the experiment. Near-infrared reflectance properties from 400 to $2500 \mathrm{~nm}$ have been measured in the laboratory for pyroxene and olivine over a temperature range of 80-448 K (Singer and Roush 

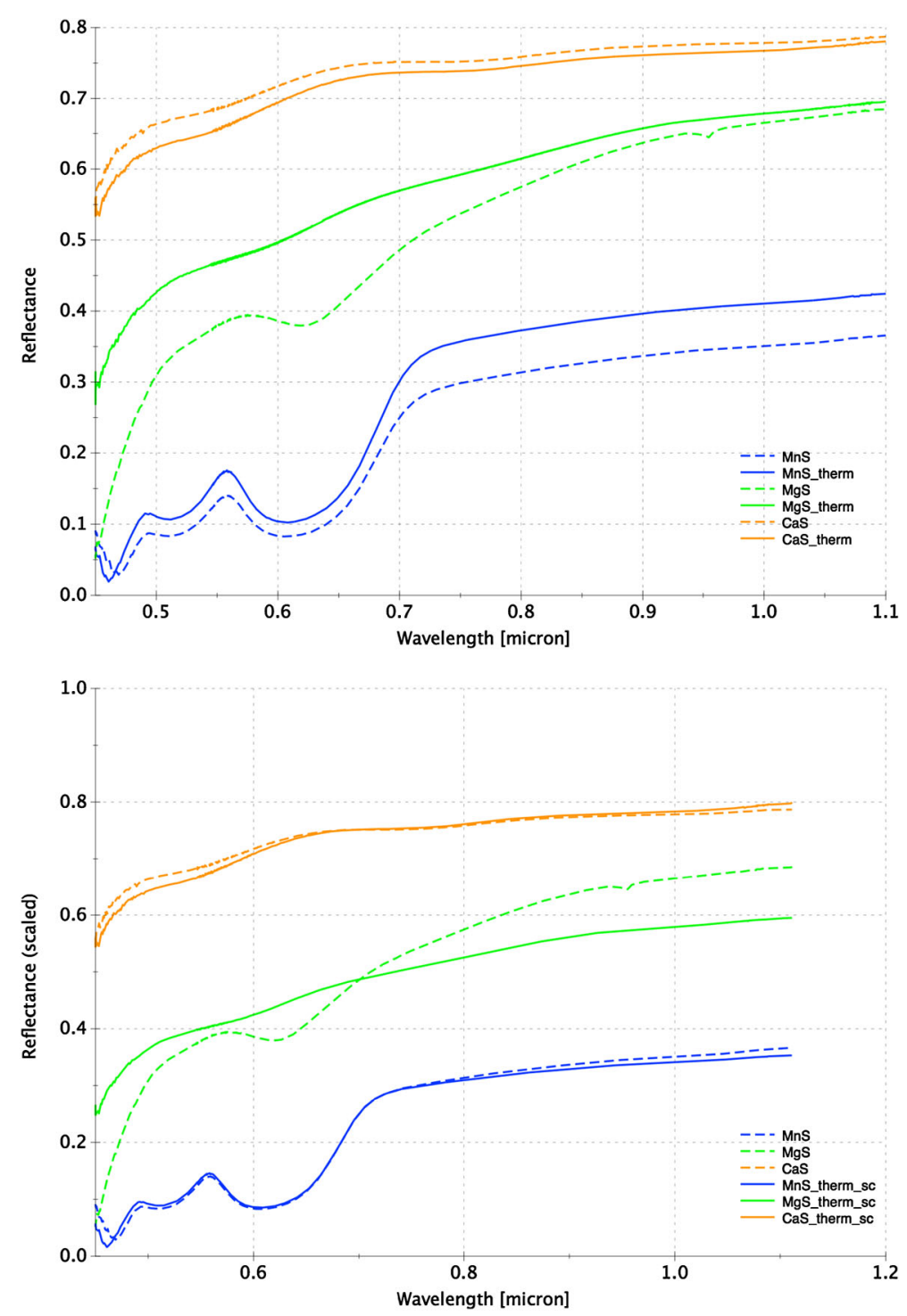

Fig. 28 Measured reflectance of synthetic sulfides CaS (tan), MgS (green), and MnS (blue) for unheated (dashed lines) and thermally cycled (solid lines) samples. The spectra in the bottom graph have been normalized to unity at $0.7 \mu \mathrm{m}$ to accent the spectral variations. The spectral changes induced by heating to Mercury dayside temperatures $\left(500^{\circ} \mathrm{C}\right)$ vary with the cation bonded with the sulfur. From Helbert et al. (2013)

1985). These measurements show that absorption features broaden with increasing temperature (Singer and Roush 1985). More recent work by Helbert et al. (2013) examined the spectral changes in synthetic sulfides that are heated to Mercury's dayside temperatures (nearly $500{ }^{\circ} \mathrm{C}$ ). These sulfides display spectral changes with heating and cooling that are dependent on the cation contained in the sulfide (Fig. 28). Calcium sulfide (oldhamite) displays the smallest variations from thermal cycling whereas $\mathrm{MgS}$ displays the largest. In all cases spectral slope and color changed after thermal cycling (Helbert et al. 2013). 
Estimates of meteoritic contributions to Mercury's regolith range between 5 and $20 \mathrm{wt} \%$ (Noble et al. 2007). This contribution would likely include carbonaceous material from both asteroidal and cometary sources, potentially at a percentage sufficiently high to contribute to the darkening of Mercury's regolith. This darkening agent would be well mixed within the regolith by impact gardening, but its global distribution is unknown. Monte Carlo simulations of radar measurements of the meteoroid flux at Earth have shown that the dominant distribution of radiants (70 \% of the flux) is centered about the apex to Earth's motion, and the remaining contributions come from radiants near the sunward and anti-sunward directions (Janches et al. 2006). Even if the micrometeoroid flux is anisotropic at $1 \mathrm{AU}$, it is unclear how the distribution of radiants at Earth would map to those at Mercury. Some latitudinal variation in exogenic (meteoritic) material might be possible. Variations in spectral reflectance and elemental composition seen among geologic terrains at a given latitude would thus, for such a darkening agent, reflect inherent differences in composition.

Models of Mercury's surface material, under the assumption that it was derived from high-degree partial melts of an enstatite chondrite source region, have provided close matches to the elemental and mineralogical chemistry of Mercury's surface with the exception of iron content (Weider et al. 2012). The models predict lower Fe content than is measured for Mercury, which may indicate an exogenic contribution from meteoroid impacts (Weider et al. 2013). Alternatively, should the Fe content be shown to be entirely endogenic, the mismatch between the measured and predicted Fe content may indicate that Mercury's surface is not derived from a pure enstatite chondrite material.

\section{Discussion}

Remote sensing of Mercury's regolith, derived from crustal bedrock, is at present the sole means for inferring the planet's crustal composition. But the regolith's original physical, chemical, and mineralogical nature has been space weathered, processed, and altered, thus complicating the interpretation of crustal mineralogical composition and modifying the elemental chemistry (including its spatial variations). To interpret these space-weathering effects on Mercury's regolith, an understanding of Mercury as an interactive system is needed. This paper has reviewed this system, tying together the exosphere formation processes, the space environment, and surface composition. The same processes that form and sustain the exosphere are responsible for the weathering of Mercury's surface. The space environment, including the solar wind and Mercury's global magnetic field and magnetosphere, exerts control over these processes.

The expected physical, chemical, and mineralogical regolith traits resulting from weathering processes are summarized in Table 12. The higher micrometeoroid flux and impact velocities on Mercury compared with those on the Moon should produce more impact melt products, such as agglutinates, glasses, and vapor coatings (rims or patinas) on regolith grains. Impacts should create and garden Mercury's regolith perhaps an order of magnitude more efficiently than the lunar regolith, readily providing new crustal material for exospheric production and burying matured and reduced material. The XRS and GRS on MESSENGER sample the regolith to different depths, yet the similarity in measured abundances for $\mathrm{Al}, \mathrm{S}$, and $\mathrm{Ca}$ indicate that Mercury's regolith is approximately homogeneous to depths of tens of centimeters (Evans et al. 2012). This observation suggests that the regolith is well gardened to such depths and has potentially reached a steady state. Both solar wind irradiation and deposition of impact vapor and melts on surficial grains create patinas that affect reflectance spectra. Mercury's magnetic field does not completely shield the surface from bombardment by solar wind and magnetospheric ions. Nightside equatorial regions are exposed to 
Table 12 Summary of Mercury surface alteration types

\begin{tabular}{|c|c|c|}
\hline Alteration type & Process & Effect \\
\hline \multirow[t]{4}{*}{ Physical/structural } & Micrometeoroid bombardment & $\begin{array}{l}\text { Greater abundance of agglutinates and } \\
\text { impact melt products compared with lunar } \\
\text { soil }\end{array}$ \\
\hline & Micrometeoroid bombardment & $\begin{array}{l}\text { Larger portion of regolith in "finest } \\
\text { fraction" }(<20 \mu \mathrm{m}) \text { compared with lunar } \\
\text { soil }\end{array}$ \\
\hline & $\begin{array}{l}\text { Solar wind irradiation, } \\
\text { micrometeoroid bombardment }\end{array}$ & $\begin{array}{l}\text { Thicker amorphous, reduced rims or } \\
\text { patinas on grains compared with lunar soil }\end{array}$ \\
\hline & Solar wind irradiation & $\begin{array}{l}\text { Increased lattice defects within regolith } \\
\text { grains compared with lunar grains }\end{array}$ \\
\hline \multirow[t]{4}{*}{ Chemical } & Ion implantation & H implantation, at saturation level? \\
\hline & Physical/chemical sputtering & Removal of $\mathrm{H}, \mathrm{OH}$ \\
\hline & $\begin{array}{l}\text { DIET, physical/chemical } \\
\text { sputtering }\end{array}$ & Depletion of alkalis \\
\hline & DIET & Repository for exospheric species \\
\hline Mineralogical & $\begin{array}{l}\text { Micrometeoroid } \\
\text { bombardment, ion } \\
\text { implantation, chemical } \\
\text { sputtering }\end{array}$ & $\begin{array}{l}\text { Reduction products, such as } n \mathrm{pFe}^{0} \text { or } \\
\text { npFeS }\end{array}$ \\
\hline
\end{tabular}

ion precipitation from the tail region, even under nominal conditions (Fig. 14). Dayside magnetospheric cusps are exposed to the solar wind under nominal conditions, increasingly so when the Sun is active. During periods of high solar activity these dayside high-latitude cusp regions can extend equatorward, exposing mid-latitude or even low-latitude regions to the solar wind. The northward shift of the magnetic dipole allows for solar wind access to a larger region in the south, creating potential space weathering differences between the two hemispheres. The proton flux at Mercury can be an order of magnitude higher than at the Moon (Table 7) when the solar wind has access to the surface. Thus, qualitatively, we expect grain surfaces at Mercury to be more highly radiation damaged, resulting in thicker or deeper amorphous layers (patinas) that contain a higher density of atomic defect sites to adsorb ions (especially from the exosphere), and a higher density of lattice defects, which act as paths for the diffusion of material from grain interiors.

Other physical alterations to Mercury's regolith include greater degrees of grinding into finer size fractions $(<45 \mu \mathrm{m})$ than observed in lunar soils by the higher micrometeoroid flux and higher impact velocities. Diffusion depletes materials from smaller grains more rapidly and therefore contributes more efficiently to the depletion of regolith in volatiles, including alkalis. Yet, the large surface area available in a finer size fraction also provides a more efficient sink for volatiles returned to the surface via exosphere- and magnetospheresurface interactions. The abundance of volatile species, such as $\mathrm{Na}, \mathrm{K}$, and $\mathrm{S}$, argues for a regolith that is an efficient sink or reservoir for such species. The anticorrelation of $\mathrm{K}$ and $\mathrm{Na}$ abundances with near-surface temperature suggests that a portion of these elements may be present at high latitudes as adsorbates within the regolith. Models of diffusion and gardening rates, however, have demonstrated that the exospheric $\mathrm{Na}$ abundances can be supplied by the surface without the need for redeposition (Killen et al. 2007).

Chemical alteration depends on both diffusion rates and lattice structural defects. The more efficient melting (compared with the Moon) produces higher amorphous content, and 
so higher diffusion rates. The greater radiation flux increases lattice structural defects, enhancing DIET and diffusion rates and creating sites for adsorption of exospheric species. Many of the processes that space weather the surface act to facilitate the transport of the more volatile elements within the regolith. The detection of such volatiles as $\mathrm{Na}$ and $\mathrm{K}$ in both the exosphere and surface argue for a dynamic interchange and transport of such species.

Space weathering changes mineralogy, for instance, through chemical reduction and depletion of alkalis (for which the efficiency depends on the relative rates of removal and implantation, as discussed above). The abundance of $\mathrm{npFe}^{0}$, a reduction process product, will depend on the initial iron content within the regolith. Even if Mercury's regolith formed from a chemically reduced mineral assemblage, iron sulfides or metal under space weathering processes will still produce $\mathrm{npFe}^{0}$ or $\mathrm{npFeS}$. These reduction products are likely the major contributors to modifications to Mercury's spectral reflectance. Assessing Mercury's surface Fe content (in silicate, oxide, sulfide, and metal forms) depends on a variety of measurement techniques, including visible and infrared measurements in which diagnostic absorption bands are diminished with increasing space weathering maturity. Neutron absorption and X-ray and gamma-ray emission features constrain Fe content but not mineralogy. Laboratory analyses of samples from asteroid Itokawa also indicate that nanophase particles that lack iron (such as npMgS) are possible (Noguchi et al. 2014), so even in a low-Fe regolith nanophase materials may be produced that will affect spectral properties and render mineral identifications difficult. Spectral modeling (Riner et al. 2009; Lucey and Riner 2011) has demonstrated that whereas opaques could darken Mercury's surface sufficiently to reduce the albedo and diminish absorption features, the quantity required for some candidate opaque minerals (e.g., Fe-Ti oxides) exceeds that permitted by the elemental abundances measured to date. These studies do not exclude the presence of a darkening material or a compositional source for some of Mercury's spectral properties, but they demonstrate that spectral alteration via space weathering is likely to have been important given the elemental abundance measurements.

Many planetary formation models have been devised to account for Mercury's high density and large core (Lewis 1988). Hypotheses include: (1) fractionation by mechanical sorting of silicate and metal grains by differential aerodynamic drag in the solar nebula at the onset of planetesimal accretion (Weidenschilling 1978), (2) preferential vaporization of silicates in the crust and upper mantle (after planetary differentiation) by a hot early solar nebula and removal of the silicate vapor by a strong early solar wind (Cameron 1985; Fegley and Cameron 1987), (3) incomplete condensation of silicates at high pressures and temperatures in the innermost nebula [because metal alloys condense at a higher temperatures than do Mg silicates (Lewis 1973)], (4) removal of the outer silicate crust and upper mantle after planetary differentiation by a giant impact (Wetherill 1988; Benz et al. 1988, 2007), and (5) condensation in an inner nebular annulus enriched in C-rich dust in which a high-temperature $(T>1000 \mathrm{~K})$, reducing environment allows metal to condense in greater abundance than olivine in addition to enabling $\mathrm{S}$ to behave as a refractory element (Ebel and Alexander 2011).

The mechanical sorting hypothesis does not selectively fractionate major silicate minerals (Lewis 1988), so there would be no relative enrichment of olivine or pyroxene, for example. Lewis (1988) concluded that the silicate portion would include 3.6-4.5 wt\% alumina, $\sim 1 \mathrm{wt} \%$ alkali oxides, and $0.5-6 \mathrm{wt} \% \mathrm{FeO}$. The vaporization mechanism would strongly enrich the present crust and upper mantle in refractory elements (e.g., $\mathrm{Al}, \mathrm{Ca}, \mathrm{Ti}, \mathrm{Th}$ ), severely deplete it in volatile elements, and fractionate Fe, Mg, and Si (Fegley and Cameron 1987; Lewis 1988). Incomplete silicate condensation models predict strong volatile depletion, enrichment in refractory elements, and low-FeO mantle compositions (Lewis 1973). The giant 
Table 13 Elemental abundances predicted by Mercury formation models

\begin{tabular}{|c|c|c|c|c|c|}
\hline $\begin{array}{l}\text { Chemical } \\
\text { signature }\end{array}$ & $\begin{array}{l}\text { Dynamical } \\
\text { nebular } \\
\text { fractionation }\end{array}$ & $\begin{array}{l}\text { Incomplete } \\
\text { silicate } \\
\text { condensation }\end{array}$ & $\begin{array}{l}\text { Mantle } \\
\text { vaporization }\end{array}$ & Giant impact & $\begin{array}{l}\text { C-rich dust } \\
\text { enrichment }\end{array}$ \\
\hline $\begin{array}{l}\text { Crustal } \\
\mathrm{FeO}\end{array}$ & $\begin{array}{l}0.5-6 \mathrm{wt} \% \\
\text { (as predicted } \\
\text { by } \\
\text { condensation } \\
\text { models) }\end{array}$ & Low & $\begin{array}{l}\text { Severely } \\
\text { depleted }\end{array}$ & $\begin{array}{l}0.5-6 \mathrm{wt} \% \\
\text { (equivalent to } \\
\text { the primordial } \\
\text { oxidation } \\
\text { state at } \\
\text { accretion) }\end{array}$ & $\begin{array}{l}\text { Low } \mathrm{FeO}, \\
\text { elevated } \mathrm{Fe} / \mathrm{Si}\end{array}$ \\
\hline $\begin{array}{l}\text { Refractory } \\
\text { elements } \\
(\mathrm{Ca}, \mathrm{Al} \text {, } \\
\text { and } \mathrm{Ti})\end{array}$ & $\begin{array}{l}3.6-4.5 \mathrm{wt} \% \\
\mathrm{Al}\end{array}$ & $\begin{array}{l}\text { Strongly } \\
\text { enriched }\end{array}$ & $\begin{array}{l}\text { Strongly } \\
\text { enriched }\end{array}$ & $\begin{array}{l}\text { No enhance- } \\
\text { ment; severe } \\
\text { depletion of } \\
\mathrm{Ca} \text { and } \mathrm{Al}\end{array}$ & Enriched \\
\hline $\begin{array}{l}\text { Alkalis } \\
(\mathrm{Na} \text { and } \mathrm{K})\end{array}$ & $\begin{array}{l}\sim 1 \mathrm{wt} \% \\
\text { (alkali } \\
\text { oxides) solar } \\
\text { values }\end{array}$ & $\begin{array}{l}\text { Severely } \\
\text { depleted }\end{array}$ & $\begin{array}{l}\text { Severely } \\
\text { depleted }\end{array}$ & $\begin{array}{l}\text { Severely } \\
\text { depleted in } \\
\text { volatile } \\
\text { alkalis metals }\end{array}$ & $\begin{array}{l}\text { Na depletion, } \\
\text { K not } \\
\text { determined }\end{array}$ \\
\hline Sulfur & $\mathrm{S}$ to core & $\begin{array}{l}\text { Severely } \\
\text { depleted }\end{array}$ & $\begin{array}{l}\text { Severely } \\
\text { depleted }\end{array}$ & Undetermined & $\begin{array}{l}\text { High S/Si } \\
\text { ratio }\end{array}$ \\
\hline
\end{tabular}

impact hypothesis might yield a secondary crust produced by mantle partial melting but depleted in $\mathrm{Ca}, \mathrm{Al}$, and volatile alkalis relative to the pre-impact crust (Lewis 1988). The extent to which volatiles would be depleted in Mercury's mantle following a giant impact is not clear, though Monte Carlo simulations suggest that some of the impact ejecta could re-accrete (Benz et al. 2007). The impact that produced the Moon strongly depleted volatiles from the material delivered to Earth orbit (from which the Moon formed), but the target body (Earth) retained much of its volatile components. Some giant impact models for Mercury call for a single, large impactor that nearly completely destroys the proto-planet and results in at most modest re-accretion of ejecta (Benz et al. 2007). Comparison with the EarthMoon giant impact suggests that for Mercury some fraction of original volatile abundances may have been retained within the post-impact planet. Silicate and $\mathrm{FeO}$ contents, however, would be determined by the oxidation state of the material present at the time and location of Mercury's accretion (Lewis 1988). This outcome would also hold for the fractionation hypothesis. Models involving formation by condensation from carbon-enriched materials predict high $\mathrm{S} / \mathrm{Si}$ and pyroxene/olivine ratios, very low silicate $\mathrm{FeO}$, and elevated bulk $\mathrm{Fe} / \mathrm{Si}$ compositions (Ebel and Alexander 2011). Table 13 summarizes the compositional variations predicted for each formation model.

$\mathrm{Mg} / \mathrm{Si}, \mathrm{Al} / \mathrm{Si}$, and $\mathrm{Ca} / \mathrm{Si}$ ratios measured from orbit by XRS and $\mathrm{K}$, Th, and $\mathrm{U}$ abundances determined by GRS allow for testing of the various formation hypotheses. $\mathrm{Mg} / \mathrm{Si}$, $\mathrm{Al} / \mathrm{Si}$, and $\mathrm{Ca} / \mathrm{Si}$ ratios are consistent with the removal of an early basaltic crust by either large-scale vaporization or a giant impact (Nittler et al. 2011). However, Mercury's surface material is not highly depleted in the volatile elements $\mathrm{S}, \mathrm{K}$, and $\mathrm{Na}$ (Nittler et al. 2011; Peplowski et al. 2011; Evans et al. 2012). Formation mechanisms that result in refractoryrich, volatile-poor compositions, such as vaporization or incomplete silicate condensation models, are thus ruled out by the MESSENGER elemental abundance data (Nittler et al. 2011; Peplowski et al. 2011). The S/Si ratio is higher than terrestrial, the K/Th ratio is terrestrial rather than depleted as is observed on the Moon (Nittler et al. 2011; 
Peplowski et al. 2011), and the abundance of Na suggests the presence of plagioclase feldspar comparatively rich in albite (Peplowski et al. 2012, 2014). The high S abundance alone may not be an indicator of volatile retention, as $\mathrm{S}$ has been shown to behave as a refractory element under highly reducing conditions (McCoy et al. 1999; Ebel and Alexander 2011). The observed K/Th ratio indicates that Mercury's ratio of volatile to refractory lithophile elements is similar to those of the other terrestrial planets (Peplowski et al. 2011), and recent studies have shown that $\mathrm{K}$ can also behave as a refractory element in C-rich systems (Ebel and Sack 2013).

Mercury's surface composition is well matched by simple partial melting of enstatite chondrite-like precursor materials (Burbine et al. 2002; Nittler et al. 2011). Ebel and Alexander (2011) suggested an origin scenario for Mercury involving enstatite-chondrite-like parent bodies and local enrichment of the nebular disk in C-rich interplanetary dust. Although these hypotheses are consistent with Mercury's high surface abundance of sulfur, they do not explain Mercury's high bulk Fe/Si ratio (Nittler et al. 2011), indicating silicate removal by either a silicate-metal fractionation process in the solar nebula or impact removal of Mercury's early crust and upper mantle (Nittler et al. 2011). Understanding the identities and relative abundances of crustal minerals is important to distinguish among the possible formation hypotheses.

As discussed above, space weathering will alter the composition of Mercury's surface materials. For example, any iron in silicates is converted to $n p \mathrm{Fe}^{0}$, thus biasing determination of crustal $\mathrm{FeO}$. Additional nanoscale materials (such as $\mathrm{FeS}$ or $\mathrm{MgS}$ ) may be present as a result of space weathering, making the original mineral hosts (not necessarily silicates) more difficult to determine. Whereas space weathering processes affect the topmost layers of regolith grains, and grain interiors retain much of the original mineral structure, it is the topmost layers that most strongly influence optical reflectance measurements, and the reason spectral observations are so highly affected by space weathering. Remote mineral identifications are predominantly made by optical reflectance measurements, so under strong space weathering elemental composition measurements are needed to provide constraints on mineral abundances. Alkalis and other volatiles can be preferentially removed from the regolith grains relative to refractories by many of the processes that accompany space weathering. Yet the detection of many of these volatile elements within the regolith of Mercury indicates that the removal of these elements from the regolith grain structure is not equivalent to the loss of these elements from the regolith. Many of these volatile species redeposit onto the fine-grained, radiation-damaged regolith grains. The abundance of the exospheric species detected to date can be produced from the regolith under current diffusion and gardening rates without the requirement for recycling. Understanding removal rates by these processes will be a critical step in better establishing the relative abundances of these species. Thus to distinguish among formation models for Mercury we must understand the influence of each weathering process over the formation history of the regolith as well as the links between the exosphere and surface.

There are specific studies that can be conducted to examine the relative roles of the various space weathering processes. Simulations of the electron and proton flux to the surface under various IMF conditions can define potential latitudinal variations in space weathering signatures. Latitudinal domains defined by these simulations and correlated with spectral properties and elemental abundances will define the magnitude to which solar wind bombardment is altering the surface. These effects, however, must be decoupled from signatures correlated to variations in near-surface temperature. Coupled studies of temperature, spectral properties, and elemental abundances are needed to establish potential adsorbate versus mineral compositions within Mercury's regolith. Laboratory spectral measurements 
of low-Fe minerals under radiation and impact environments are needed to better establish the variations in space weathering products and their corresponding influence on reflectance properties. Similar laboratory measurements are needed for sulfur-containing mineral assemblages. As detailed in this paper, our current understanding of the effects of Mercury's environment on chemical and mineralogical remote sensing of its surface material illuminates the many questions still to be answered.

Acknowledgements The authors deeply appreciate the efforts and dedication of the MESSENGER mission operations, engineering, and instrument teams, without whom the scientific successes of the MESSENGER mission would not have been possible. The MESSENGER project is supported by the NASA Discovery Program under contracts NASW-00002 to the Carnegie Institution of Washington and NAS5-97271 to The Johns Hopkins University Applied Physics Laboratory. Contributions by D.T.B., D.S., M.B, and R.K. are made possible by grants from the NASA MESSENGER Participating Scientist Program (NNX08AN29G, NNX07AR62GO, NNX07AR61G, and NNX07AR78G, respectively). The authors also thank T.B. McCord and an anonymous reviewer for their comments on an earlier version of this manuscript.

Open Access This article is distributed under the terms of the Creative Commons Attribution License which permits any use, distribution, and reproduction in any medium, provided the original author(s) and the source are credited.

\section{References}

M.M. Abbas, D. Tankosic, P.D. Craven, A.C. LeClair, J.F. Spann, Astrophys. J. 718, 795 (2010)

J. Adams, R. Jones, Science 167, 737 (1970)

M.R. Aellig, A.J. Lazarus, J.T. Steinberg, Geophys. Res. Lett. 28, 2767 (2001)

E. Anders, Icarus 24, 363 (1975)

B.J. Anderson, M.H. Acuña, H. Korth, M.E. Purucker, C.L. Johnson, J.A. Slavin, S.C. Solomon, R.L. McNutt Jr., Science 321, 82 (2008)

B.J. Anderson, M.H. Acuña, H. Korth, J.A. Slavin, H. Uno, C.L. Johnson, M.E. Purucker, S.C. Solomon, J.M. Raines, T.H. Zurbuchen, G. Gloeckler, R.L. McNutt Jr., Space Sci. Rev. 152, 307 (2010)

B.J. Anderson, C.L. Johnson, H. Korth, M.E. Purucker, R.M. Winslow, J.A. Slavin, S.C. Solomon, R.L. McNutt Jr., J.M. Raines, T.H. Zurbuchen, Science 333, 1859 (2011)

Apollo Soil Survey, Earth Planet. Sci. Lett. 12, 49 (1971)

M. Audard, M. Gudel, J.J. Drake, V.L. Kashyap, Astrophys. J. 541, 396 (2000)

R.L. Baron, E. Bilson, T. Gold, R.J. Colton, B. Hapke, M.A. Steggert, Earth Planet. Sci. Lett. 37, 263 (1977)

R.L. Baron, E. Bilson, T. Gold, R.J. Colton, B. Hapke, M.A. Steggert, Earth Planet. Sci. Lett. 41, 2471 (1978)

D.N. Baker, D. Odstrcil, B.J. Anderson, C.N. Arge, M. Benna, G. Gloeckler, J.M. Raines, D. Schriver, J.A. Slavin, S.C. Solomon, R.M. Killen, T.H. Zurbuchen, J. Geophys. Res. 114, A10101 (2009). doi:10.1029/ 2009JA014287

S.J. Bame, J.R. Asbridge, W.C. Feldman, J.T. Gosling, J. Geophys. Res. 82, 1487 (1977)

A. Basu, J. Sediment. Petrol. 46, 694 (1976)

A. Basu, D.S. McKay, Meteoritics 18, 263 (1983)

A. Basu, D.J. DesMarais, J.M. Hayes, W.G. Meinschein, Moon 14, 129 (1975)

D. Bäuerle, Laser Processing and Chemistry (Springer, New York, 2000). 788 pp.

J. Beer, M. Vonmoos, R. Muscheler, Space Sci. Rev. 125, 67 (2006)

R. Behrisch, K. Wittmaack, in Sputtering by Particle Bombardment III, ed. by R. Behrisch, K. Wittmaack (Springer, New York, 1991), pp. 1-13

M. Benna, B.J. Anderson, D.N. Baker, S.A. Boardsen, G. Gloeckler, R.E. Gold, G.C. Ho, R.M. Killen, H. Korth, S.M. Krimigis, M.E. Purucker, R.L. McNutt Jr., J.M. Raines, W.E. McClintock, M. Sarantos, J.A. Slavin, S.C. Solomon, T.H. Zurbuchen, Icarus 209, 3 (2010)

W. Benz, W.L. Slattery, A.G.W. Cameron, Icarus 74, 516 (1988)

W. Benz, A. Anic, J. Horner, J.A. Whitby, Space Sci. Rev. 132, 189 (2007)

A.A. Berezhnoy, B.A. Klumov, Icarus 195, 511 (2008)

S.K. Bhattacharya, J.N. Goswami, D. Lat, P.P. Patel, M.N. Rao, Proc. Lunar Sci. Conf. 6, 3509 (1975)

T.A. Bida, R.M. Killen, T.H. Morgan, Nature 404, 159 (2000)

R.P. Binzel, S.J. Bus, T.H. Burbine, J.M. Sunshine, Science 273, 946-948 (1996)

R.P. Binzel, A.S. Rivkin, S.J. Bus, J.M. Sunshine, T.H. Burbine, Meteorit. Planet. Sci. 36, 1167 (2001) 
C. Björkas, K. Vörtler, D. Nishijima, R. Doerner, New J. Phys. 11, 123017 (2009)

D.T. Blewett, P.G. Lucey, B. Ray Hawke, G.G. Ling, M.S. Robinson, Icarus 129, 217 (1997)

D.T. Blewett, M.S. Robinson, B.W. Denevi, J.J. Gillis-Davis, J.W. Head, S.C. Solomon, G.M. Holsclaw, W.E. McClintock, Earth Planet. Sci. Lett. 285, 272 (2009)

D.T. Blewett, B.W. Denevi, M.S. Robinson, C.M. Ernst, M.E. Purucker, S.C. Solomon, Icarus 209, 239 (2010)

D.T. Blewett, E.I. Coman, B.R. Hawke, J.J. Gillis-Davis, M.E. Purucker, C.G. Hughes, J. Geophys. Res. 116, E02002 (2011). doi:10.1029/2010JE003656

D.T. Blewett, W.M. Vaughan, Z. Xiao, N.L. Chabot, B.W. Denevi, C.M. Ernst, J. Helbert, M. D’Amore, A. Maturilli, J.W. Head, S.C. Solomon, J. Geophys. Res. Planets 118, 1013 (2013). doi:10.1029/ 2012JE004174

P. Bochsler, Space Sci. Rev. 85, 291 (1998)

J. Borg, M. Maurette, L. Durrieu, C. Jouret, Proc. Lunar Sci. Conf. 2, 2027 (1971)

P. Borin, G. Cremonese, F. Marzari, M. Bruno, S. Marchi, Astron. Astrophys. 503, 259 (2009)

P. Borin, M. Bruno, G. Cremonese, F. Marzari, Astron. Astrophys. 517, 5 (2010). doi:10.1051/0004-6361/ 201014312

W.F. Bottke, D. Vokrouhlicky, D. Minton, D. Nesvorny, A. Morbidelli, R. Brasser, B. Simonson, H.F. Levison, Nature 485, 78 (2012)

W.V. Boynton, A.L. Sprague, S.C. Solomon, R.D. Starr, L.G. Evans, W.C. Feldman, J.I. Trombka, E.A. Rhodes, Space Sci. Rev. 131, 85 (2007)

S.M. Brown, L.T. Elkins-Tanton, Earth Planet. Sci. Lett. 286, 446 (2009)

M. Broz, D. Vokrouhlicky, A. Morbidelli, D. Nesvorny, W.F. Bottke, Mon. Not. R. Astron. Soc. 414, 2716 (2011). doi:10.1111/j.1365-2966.2011.18587.x

R. Brunetto, G. Strazzulla, Icarus 179, 265 (2005)

R. Brunetto, F. Romano, A. Blanco, S. Fonti, M. Martino, V. Orofino, C. Verrienti, Icarus 180, 546 (2006a)

R. Brunetto, P. Vernazza, S. Marchi, M. Birlan, M. Fulchignoni, V. Orofino, G. Strazzulla, Icarus 184, 327 (2006b)

R. Brunetto, T. Roush, A.C. Marra, V. Orofino, Icarus 191, 381 (2007)

T.H. Burbine, T.J. McCoy, L.R. Nittler, G.K. Benedix, E.A. Cloutis, T.L. Dickinson, Meteorit. Planet. Sci. 37, 1233 (2002)

M.H. Burger, R.M. Killen, R.J. Vervack, E.T. Bradley, W.E. McClintock, M. Sarantos, M. Benna, N. Mouawad, Icarus 209, 63 (2010)

R.G. Burns, in Remote Geochemical Analysis, ed. by C.M. Pieters, P.A.J. Englert (Cambridge University Press, New York, 1993), pp. 3-29

A.G.W. Cameron, Icarus 64, 285 (1985)

T.A. Cassidy, R.E. Johnson, Icarus 176, 499 (2005)

C.R. Chapman, Meteorit. Planet. Sci. 31, 699 (1996)

C.R. Chapman, Annu. Rev. Earth Planet. Sci. 32, 539 (2004)

C.R. Chapman, J.W. Salisbury, Icarus 19, 507 (1973)

C.R. Chapman, B.A. Cohen, D.H. Grinspoon, Icarus 189, 233 (2007)

S. Charnoz, A. Morbidelli, L. Dones, J. Salmon, Icarus 199, 413 (2009)

S.C. Chase, E.D. Miner, D. Morrison, G. Munch, G. Neugebauer, M. Schroeder, Science 185, 142 (1974)

S.C. Chase, E.D. Miner, D. Morrison, G. Munch, G. Neugebauer, Icarus 28, 565 (1976)

H. Chen, A. Aleksandrov, Y. Chen, S. Zha, M. Liu, T.M. Orlando, J. Phys. Chem. B 109, 11257 (2005)

D.B. Chrisey, G.K. Hubler (eds.), Pulsed Laser Deposition of Thin Films (Wiley, New York, 1994), 648 pp.

M.J. Cintala, J. Geophys. Res. 97, 947 (1992)

B.E. Clark, B. Hapke, C. Pieters, D. Britt, in Asteroids III, ed. by W.K. Bottke Jr., A. Celliono, P. Paolicchi, R.P. Binzel (University of Arizona Press, Tucson, 2002), pp. 585-599

R.N. Clark, Science 326, 562 (2009)

B.A. Cohen, T.D. Swindle, D.A. Kring, Science 290, 1754 (2000)

P.J. Coleman Jr., G. Schubert, C.T. Russell, L.R. Sharp, Moon 4, 419 (1972)

J.E. Colwell, S. Batiste, M. Horányi, S. Robertson, S. Sture, Rev. Geophys. 45, RG2006 (2007). doi:10.1029/ 2005RG000184

D.H. Crider, R.R. Vondrak, J. Geophys. Res. 108, 5079 (2003). doi:10.1029/2002JE002030

D.R. Criswell, in Photon and Particle Interactions with Surfaces in Space, ed. by R.J.L. Grard (Reidel, Dordrecht, 1973), pp. 545-556

A. Czechowski, I. Mann, Astrophys. J. 714, 89 (2010)

M.E. Davies, D.E. Dwornik, D.E. Gault, R.G. Strom, Atlas of Mercury (U.S. Government Printing Office, Washington, 1978), $128 \mathrm{pp}$.

C. Davoisne, H. Leroux, M. Frere, J. Gimblot, L. Gengembre, Z. Djouadi, V. Ferreiro, L. D'Hendecourt, A. Jones, Astron. Astrophys. 482, 541 (2008). doi:10.1051/0004-6361:20078964 
J.W. Delano, J. Geophys. Res. 91, D201 (1986)

B.W. Denevi, M.S. Robinson, Icarus 197, 239 (2008)

B.W. Denevi, M.S. Robinson, S.C. Solomon, S.L. Murchie, D.T. Blewett, D.L. Domingue, T.J. McCoy, C.M. Ernst, J.W. Head, T.R. Watters, N.L. Chabot, Science 324, 613 (2009)

B.W. Denevi, C.M. Ernst, H.M. Meyer, M.S. Robinson, S.L. Murchie, J.L. Whitten, J.W. Head, T.R. Watters, S.C. Solomon, L.R. Ostrach, C.R. Chapman, P.K. Byrne, C. Klimczak, P.N. Peplowski, J. Geophys. Res., Planets 118, 891 (2013). doi:10.1002/jgre.20075

M.I. Desai, G.M. Mason, J.E. Mazur, J.R. Dwyer, Astrophys. J. Lett. 645, L81 (2006)

D.J. DesMarais, J.M. Hayes, W.G. Meinschein, Proc. Lunar Sci. Conf. 5, 1811 (1974)

M.C. De Sanctis, E. Ammannito, M.T. Capria, F. Tosi, F. Capaccioni, F. Zambon, F. Carraro, S. Fonte, A. Frigeri, R. Jaumann, G. Magni, S. Marchi, T.B. McCord, L.A. McFadden, H.Y. McSween, D.W. Mittlefehldt, A. Nathues, E. Palomba, C.M. Pieters, C.A. Raymond, C.T. Russell, M.J. Toplis, D. Turrini, Science 336, 697 (2012)

J.M. Devine, D.S. McKay, J.J. Papike, J. Geophys. Res. 87, A260 (1982)

Yu.P. Dikov, O.A. Bogatikov, V.L. Barsukov, K.P. Florensky, A.V. Ivanov, V.V. Nemoshkalenko, V.G. Aleshin, M.G. Chudinov, Proc. Lunar Planet. Sci. Conf. 9, 2111 (1978)

D.L. Domingue, M. Robinson, B. Carcich, J. Joseph, P. Thomas, B.E. Clark, Icarus 155, 205 (2002)

D.L. Domingue, P.L. Koehn, R.M. Killen, A.L. Sprague, M. Sarantos, A.F. Cheng, E.T. Bradley, W.E. McClintock, Space Sci. Rev. 131, 161 (2007)

D.L. Domingue, F. Vilas, G.M. Holsclaw, J. Warell, N.R. Izenberg, S.L. Murchie, B.W. Denevi, D.T. Blewett, W.E. McClintock, B.J. Anderson, M. Sarantos, Icarus 209, 101 (2010)

D.L. Domingue, S.L. Murchie, N.L. Chabot, B.W. Denevi, F. Vilas, Planet. Space Sci. 59, 1853 (2011). doi:10.106/j.pss.2011.04.012

K.A. Drake, R.A. Heelis, M.R. Hairston, P.C. Anderson, J. Geophys. Res. 114, A04215 (2009). doi:10.1029/ 2008JA013608

C.A. Dukes, R.A. Baragiola, L.A. McFadden, J. Geophys. Res. 104, 1865 (1999). doi:10.1029/98JE02820

W.W. Duley, D.A. Williams, Mon. Not. R. Astron. Soc. 260, 37 (1993)

D.S. Ebel, in Meteorites and the Early Solar System II, ed. by D.S. Lauretta, H.Y. McSween Jr., R.P. Binzel (University of Arizona Press, Tucson, 2006), pp. 253-277

D.S. Ebel, C.M.O’D Alexander, Planet. Space Sci. 59, 1888 (2011). doi:10.1016/j.pss.2011.07.017

D.S. Ebel, R.O. Sack, Contrib. Mineral. Petrol. 166, 923 (2013)

C. Elachi, Adv. Space Res. 7, 249 (1987)

F. El-Baz, Apollo 16 prelim. Sci. Rept., NASA SP-315, 29-93 (1972)

J.P. Emery, A.L. Sprague, F.C. Witteborn, J.E. Colwell, R.W.H. Kozlowski, D.H. Wooden, Icarus 136, 104 (1998)

S. Epstein, H.P. Taylor Jr., Proc. Lunar Sci. Conf. 3, 1429 (1972)

S. Epstein, H.P. Taylor Jr., Proc. Lunar Sci. Conf. 6, 1771 (1975)

L.G. Evans, P.N. Peplowski, E.A. Rhodes, D.J. Lawrence, L.R. Nittler, T.J. McCoy, A.L. Sprague, K.R. Stockstill-Cahill, R.D. Starr, S.Z. Weider, W.V. Boynton, D.K. Hamara, S.C. Solomon, J. Geophys. Res. 117, E00L07 (2012). doi:10.1029/2012JE004178

P. Evenson, M. Garcia-Munoz, P. Meyer, K.R. Pyle, J.A. Simpson, Astrophys. J. Lett. 275, L15 (1983)

O. Eugster, G.F. Herzog, K. Marti, M.W. Caffee, in Meteorites and Early Solar System II, ed. by D.S. Lauretta, H.Y. McSween Jr. (University of Arizona Press, Tucson, 2006), pp. 829-851

C.I. Fassett, J.W. Head, S.J. Kadish, E. Mazarico, G.A. Neumann, D.E. Smith, M.T. Zuber, J. Geophys. Res. 117, E00H06 (2012). doi:10.1029/2011JE003951

W.M. Farrell, T.J. Stubbs, R.R. Vondrak, G.T. Delory, J.S. Halekas, Geophys. Res. Lett. 34, L14201 (2007). doi:10.1029/2007GL029312

W.M. Farrell, T.J. Stubbs, G.T. Delory, R.R. Vondrak, M.R. Collier, J.S. Halekas, R.P. Lin, Geophys. Res. Lett. 35, L19104 (2008). doi:10.1029/2008GL034785

B. Fegley, A.G.W. Cameron, Earth Planet. Sci. Lett. 82, 207 (1987)

W.C. Feldman, D.J. Lawerence, R.C. Elphic, B.L. Barrachlough, S. Maurice, I. Genetay, A.B. Binder, J. Geophys. Res. 105, 4175 (2000)

E.M. Fischer, C.M. Pieters, Icarus 111, 475 (1994)

L.A. Fisk, G. Gloeckler, Astrophys. J. 686, 1466 (2008)

L.A. Fisk, G. Gloeckler, N.A. Schwadron, Astrophys. J. 720, 533 (2010)

L.A. Fisk, N.A. Schwadron, T.H. Zurbuchen, Space Sci. Rev. 86, 51 (1998)

C.N. Foley, L.R. Nittler, T.J. McCoy, L.F. Lim, M.R.M. Brown, R.D. Starr, J.I. Trombka, Icarus 184, 338 (2006). doi:10.1016/j.icarus.2006.05.011

P.C. Frisch, J.D. Slavin, Astrophys. Space Sci. Trans. 2, 53 (2006)

D. Fulvio, R. Brunetto, P. Vernazza, G. Strazzulla, Astron. Astrophys. 537, L11 (2012). doi:10.1051/ 0004-6361/201118486 
L.R. Gaddis, M.I. Staid, J.A. Tyburczy, B.R. Hawke, N.E. Petro, Icarus 161, 262 (2003)

I. Garrick-Bethell, J.W. Head, C.M. Pieters, Icarus 212, 480 (2011)

J. Geiss, in Proc. 13th Int. Cosmic Ray Conf., vol. 5 (1973), p. 3375

J. Geiss, Lunar Interactions: Abstracts of Papers Presented at the Conference on Interactions of the Interplanetary Plasma with the Modern and Ancient Moon. LPI Contribution, vol. 195 (Lunar and Planetary Institute, Houston, 1974), p. 110

J. Geiss, G. Gloeckler, R. von Steiger, Space Sci. Rev. 72, 49 (1995)

B. Gladman, J. Coffey, Meteorit. Planet. Sci. 44, 285 (2009)

G.R. Gladstone, D.M. Hurley, K.D. Retherford, P.D. Feldman, W.R. Pryor, J.-Y. Chaufray, M. Versteeg, T.K. Greathouse, A.J. Steffl, H. Throop, J.W. Parker, D.E. Kaufmann, A.F. Egan, M.W. Davis, D.C. Slater, J. Mukherjee, P.F. Miles, A.R. Hendrix, A. Collaprete, A.S. Stern, Science 330, 472 (2010)

L.J. Gleeson, W.I. Axford, Astrophys. J. 154, 1011 (1968)

H. Gleisner, J. Watermann, Space Weather 4, S06006 (2006). doi:10.1029/2006SW000220

D.A. Glenar, T.J. Stubbs, W.M. Farrell, J.W. Keller, R.R. Vondrak, in Annual Meeting of the Lunar Exploration Analysis Group, Abstract 3008 (Lunar and Planetary Institute, Houston, 2012)

G. Gloeckler, in Solar Wind 10: AIP Conf. Proc., vol. 679 (2003), p. 583

G. Gloeckler, J. Geiss, Space Sci. Rev. 97, 169 (2001)

G. Gloeckler, J. Geiss, E.C. Roelof, L.A. Fisk, F.M. Ipavich, K.W. Ogilvie, L.J. Lanzerotti, R. von Steiger, B. Wilken, J. Geophys. Res. 99, 17637 (1994)

G. Gloeckler, T.H. Zurbuchen, J. Geiss, J. Geophys. Res. 108, 1158 (2003). doi:10.1029/2002JA009286

T. Gold, M. Champbell, B.T. O'Leary, in Proc. Apollo 11 Lunar Sci. Conf. (1970), p. 2149

T. Gold, E. Bilson, R.L. Baron, Proc. Lunar Sci. Conf. 5, 2413 (1974)

T. Gold, E. Bilson, R.L. Baron, Proc. Lunar Sci. Conf. 6, 3285 (1975)

T. Gold, E. Bilson, R.L. Baron, Proc. Lunar Sci. Conf. 7, 901 (1976)

J.T. Gosling, J. Birn, M. Hesse, Geophys. Res. Lett. 22, 869 (1995)

N. Grevesse, A.J. Sauval, Adv. Space Res. 30, 3 (2002)

D.M. Gruen, B. Siskind, R.B. Wright, J. Chem. Phys. 65, 363 (1976)

M. Güdel, Living Rev. Solar Phys. 4 (2007)

E.F. Guinan, S.G. Engle, in The Ages of Stars, ed. by E.E. Murnajek, D. Soderblom. Proceedings of IAU Symposium, vol. 258 (2009), p. 395

J.S. Halekas, D.L. Mitchell, R.P. Lin, L.L. Hood, M.H. Acuña, A.B. Butler, Geophys. Res. Lett. 29, 1435 (2002). doi:10.1029/2001GL014428

B. Hapke, Ann. N.Y. Acad. Sci. 123, 711 (1965)

B. Hapke, in The Nature of the Lunar Surface, ed. by W. Heiss, D. Menzel, J. O'Keefe (Johns Hopkins University Press, Baltimore, 1966), pp. 141-154

B. Hapke, Science 159, 76 (1968)

B. Hapke, Moon 7, 342 (1973)

B. Hapke, Phys. Earth Planet. Inter. 15, 264 (1977)

B. Hapke, J. Geophys. Res. 68, 4571 (1981)

B. Hapke, Icarus 59, 41 (1984)

B. Hapke, Icarus 67, 264 (1986)

B. Hapke, J. Geophys. Res. 106, 10039 (2001)

B. Hapke, Icarus 157, 523 (2002)

B. Hapke, A. Cohen, W. Cassidy, E. Wells, in Proc. Apollo 11 Lunar Sci. Conf. (1970), p. 2199

B. Hapke, W. Cassidy, E. Wells, Moon 13, 339 (1975)

J.K. Harmon, Space Sci. Rev. 132, 307 (2007)

J.K. Harmon, M.A. Slade, Science 258, 640 (1992)

J.K. Harmon, M.A. Slade, B.J. Butler, J.W. Head, M.S. Rice, D.B. Campbell, Icarus 187, 374 (2007)

R.E. Hartle, R.M. Killen, Geophys. Res. Lett. 33, L05201 (2006). doi:10.1029/2005GL024520

B. Hartmann, D. Domingue, Icarus 131, 421 (1998)

W.K. Hartmann, G. Ryder, L. Dones, D. Grinspoon, in Origin of the Earth and Moon, ed. by R.M. Canup, K. Righter (University of Arizona Press, Tucson, 2000), pp. 493-512

L. Haskin, P. Warren, in Lunar Sourcebook, ed. by G.H. Heiken, D.T. Vaniman, B.M. French (Cambridge University Press, New York, 1991), pp. 357-474

S.E. Hawkins III, J.D. Boldt, E.H. Darlington, R. Espiritu, R.E. Gold, B. Gotwols, M.P. Matthew, C.D. Hash, J.R. Hayes, S.E. Jaskulek, C.J. Kardian, M.R. Keller, E.R. Malaret, S.L. Murchie, P.K. Murphy, K. Peacock, L.M. Prockter, R.A. Reiter, M.S. Robinson, E.D. Schaefer, R.G. Shelton, R.E. Sterner, H.W. Taylor, T.R. Watters, B.D. Williams, Space Sci. Rev. 131, 247 (2007). doi:10.1007/s11214-007-9266-3

J.W. Head, S.L. Murchie, L.M. Prockter, M.S. Robinson, S.C. Solomon, R.G. Strom, C.R. Chapman, T.R. Watters, W.E. McClintock, D.T. Blewett, J.J. Gillis-Davis, Science 321, 69 (2008) 
J.W. Head, S.L. Murchie, L.M. Prockter, S.C. Solomon, C.R. Chapman, R.G. Strom, T.R. Watters, D.T. Blewett, J.J. Gillis-Davis, C.I. Fassett, J.L. Dickson, G.A. Morgan, L. Kerber, Earth Planet. Sci. Lett. 285, 227 (2009)

G.H. Heiken, Rev. Geophys. Space Phys. 13, 567 (1975)

G.H. Heiken, D.T. Vaniman, B.M. French, Lunar Sourcebook (Cambridge University Press, New York, 1991). 736 pp.

J. Helbert, A. Maturilli, Earth Planet. Sci. Lett. 285, 347 (2009)

J. Helbert, A. Maturilli, M. D'Amore, Earth Planet. Sci. Lett. 369, 233 (2013). doi:10.1016/ j.espl.2013.03.045

P. Helfenstein, J. Veverka, Icarus 72, 342 (1987)

P. Helfenstein, J. Veverka, in Asteroids II, ed. by R. Binzel, T. Gehrels, M.S. Matthews (University of Arizona Press, Tucson, 1989), pp. 557-593

P. Helfenstein, J. Veverka, P.C. Thomas, D.P. Simonelli, P. Lee, K. Klaasen, T.V. Johnson, H. Breneman, F. Fanale, M. Robinson, B. Clark, J. Granahan, H. Garbeil, A.S. McEwen, R.L. Kirk, M. Davies, G. Neukum, S. Mottola, R. Wagner, M. Belton, C. Chapman, C. Pilcher, Icarus 107, 37 (1994)

P. Helfenstein, J. Veverka, P.C. Thomas, D.P. Simonelli, K. Klaasen, T.V. Johnson, F. Fanale, J. Granahan, A.S. McEwen, M. Belton, C. Chapman, Icarus 120, 48 (1996)

A.R. Hendrix, F. Vilas, Astron. J. 132, 1396 (2006). doi:10.1086/506426

A.R. Hendrix, K.D. Retherford, G.R. Gladstone, D.M. Hurley, P.D. Feldman, A.F. Egan, D.E. Kaufmann, P.F. Miles, J.W. Parker, D. Horvath, P.M. Rojas, M.H. Versteeg, M.W. Davis, T.K. Greathouse, J. Mukherjee, A.J. Steffl, W.R. Pryor, S.A. Stern, J. Geophys. Res. 117, E12001 (2012). doi:10.1029/2012JE004252

H. Hiesinger, R. Jaumann, G. Neukam, J.W. Head, J. Geophys. Res. 105, 29239 (2000)

H. Hiesinger, J.W. Head, U. Wolf, R. Jaumann, G. Neukam, J. Geophys. Res. 108, 5065 (2003). doi:10.1029/ 2002JE001985

T. Hiroi, S. Sasaki, Meteorit. Planet. Sci. 36, 1587 (2001)

T. Hiroi, M. Abe, K. Kitazato, S. Abe, B.E. Clark, S. Sasaki, M. Ishiguro, O.S. Barnouin-Jha, Nature 443, 56 (2006). doi:10.1038/nature05073

G.C. Ho, R.D. Starr, R.E. Gold, S.M. Krimigis, J.A. Slavin, D.N. Baker, B.J. Anderson, R.L. McNutt Jr., L.R. Nittler, S.C. Solomon, Planetary Space Sci. 59, 2016-2025 (2011a). doi:10.1016/j.pss.2011.01.011

G.C. Ho, S.M. Krimigis, R.E. Gold, D.N. Baker, J.A. Slavin, B.J. Anderson, H. Korth, R.D. Starr, D.J. Lawrence, R.L. McNutt Jr., S.C. Solomon, Science 333, 1865 (2011b)

L. Holmlid, Planet. Space Sci. 54, 101 (2006)

L.L. Hood, G. Schubert, Science 208, 49 (1980)

L.L. Hood, C.R. Williams, Proc. Lunar Planet. Sci. Conf. 19, 99 (1989)

L.L. Hood, P.J. Coleman Jr., D.E. Wilhelms, Proc. Lunar Planet. Sci. Conf. 10, 2235 (1979a)

L.L. Hood, P.J. Coleman Jr., D.E. Wilhelms, Science 204, 53 (1979b)

L.L. Hood, C.T. Russell, P.J. Coleman Jr., J. Geophys. Res. 86, 1055 (1981)

F. Hörz, M. Cintala, Meteoritics 32, 179 (1997)

F. Hörz, M. Cintala, T.H. See, F. Cardenas, T.D. Thompson, J. Geophys. Res. 89, C183 (1984)

K.R. Housen, Proc. Lunar Planet. Sci. Conf. 12, 1717 (1982)

K.R. Housen, L.L. Wilkening, C.R. Chapman, R. Greenberg, Icarus 39, 317 (1979)

R.M. Housley, R.W. Grant, Proc. Lunar Sci. Conf. 6, 3269 (1975)

R.M. Housley, R.W. Grant, Proc. Lunar Sci. Conf. 7, 881 (1976)

R.M. Housley, R.W. Grant, Proc. Lunar Sci. Conf. 8, 3885 (1977)

R.M. Housley, E.H. Cirlin, R.W. Grant, Proc. Lunar Sci. Conf. 4, 2729 (1973a)

R.M. Housley, R.W. Grant, N.E. Patton, Proc. Lunar Sci. Conf. 4, 2737 (1973b)

R.L. Huguenin, in Water in Planetary Regoliths, ed. by S.L. Bowen, E. Wright (Dartmouth College, Hanover, 1976), pp. 100-106

F.M. Ipavich, A.B. Galvin, S.E. Lasley, J.A. Paquette, S. Hefti, K.-U. Reiche, M.A. Coplan, G. Gloeckler, P. Bochsler, D. Hovestadt, H. Grünwaldt, M. Hilchenback, F. Gliem, W.I. Axford, H. Balsiger, A. Bürgi, J. Geiss, K.C. Hsieh, R. Kallenback, B. Klecker, M.A. Lee, G.G. Managadze, E. Marsch, E. Möbius, M. Neugebauer, M. Scholer, M.I. Verigin, B. Wilken, P. Wurz, J. Geophys. Res. 103, 17205 (1998)

M. Ishiguro, T. Hiroi, D.J. Tholen, S. Sasaki, Y. Ueda, T. Nimura, M. Abe, B.E. Clark, A. Yamamoto, F. Yoshida, R. Nakamura, N. Hirata, H. Miyamoto, Y. Yokota, T. Hashimoto, T. Kubota, A.M. Nakamura, R.W. Gaskell, J. Saito, Meteorit. Planet. Sci. 42, 1791 (2007)

N.R. Izenberg, G.M. Holsclaw, D.L. Domingue, W.E. McClintock, R.L. Kilma, D.T. Blewett, M.C. Kochte, J. Helbert, M. D’Amore, A.L. Sprague, F. Vilas, S.C. Solomon, Lunar Planet. Sci. 43, 2365 (2012)

N.R. Izenberg, R.L. Klima, S.L. Murchie, D.T. Blewett, G.M. Holsclaw, W.E. McClintock, E. Malaret, C. Mauceri, F. Vilas, A.L. Sprague, J. Helbert, D.L. Domingue, J.W. Head III, T.A. Goudge, S.C. Solomon, C.A. Hibbits, M.D. Dyar, Icarus 228, 364 (2014)

R. Jeanloz, D.L. Mitchell, A.L. Sprague, I. dePater, Science 268, 1455 (1995) 
D. Janches, C.J. Heinselman, J.L. Chau, A. Chandran, R. Woodman, J. Geophys. Res. 111, A07317 (2006). doi:10.1029/2006JA011628

C.L. Johnson, M.E. Purucker, H. Korth, B.J. Anderson, R.M. Winslow, M.M.H. Al Asad, J.A. Slavin, I.I. Alexeev, R.J. Phillips, M.T. Zuber, S.C. Solomon, J. Geophys. Res. 117, E00L14 (2012). doi:10.1029/2012E004217

R.E. Johnson, R. Baragiola, Geophys. Res. Lett. 18, 2169 (1991)

R.E. Johnson, F. Leblanc, B.V. Yakshinskiy, T.E. Madey, Icarus 156, 136 (2002)

J.R. Jokipii, M.A. Lee, Astrophys. J. 713, 475 (2010)

S. Jovanovic, G.W. Reed Jr., Earth Planet. Sci. Lett. 16, 257 (1972)

S. Jovanovic, G.W. Reed Jr., Proc. Lunar Planet. Sci. Conf. 10, 636 (1979a)

S. Jovanovic, G.W. Reed Jr., Proc. Lunar Planet. Sci. Conf. 10, 1425 (1979b)

K. Kabin, T.I. Gombosi, D.L. DeZeeuw, K.G. Powell, Icarus 143, 397 (2000)

M.L. Kaiser, K. Goetz, C. Stcyr, S. Bale, M. Maksimovic, Eos Trans. AGU, Fall Meeting Suppl., Abstract SH52B-01 (2007)

E. Kallio, P. Janhunen, Ann. Geophys. 21, 2133 (2003). doi:10.5194/angeo-21-2133-2003

S.J. Keihm, M.G. Langseth, Proc. Lunar Sci. Conf. 4, 2503 (1973)

K. Keil, H. Haack, E.R.D. Scott, Planet. Space Sci. 42, 1109 (1994)

L.P. Keller, S.P. Clemett, Lunar Planet. Sci. 32, 2097 (2001)

L.P. Keller, D.S. McKay, Science 261, 1305 (1993)

L.P. Keller, D.S. McKay, Geochim. Cosmochim. Acta 61, 2331 (1997)

L.P. Keller, S.J. Wentworth, D.S. McKay, L.A. Taylor, C. Pieters, R.V. Morris, Lunar Planet. Sci. 31, 1655 (2000)

C.E. KenKnight, D.L. Rosenerg, G.K. Wehner, J. Geophys. Res. 72, 3105 (1967)

L. Kerber, J.W. Head, S.C. Solomon, S.L. Murchie, D.T. Blewett, L. Wilson, Earth Planet. Sci. Lett. 285,263 (2009)

L. Kerber, J.W. Head, D.T. Blewett, S.C. Solomon, L. Wilson, S.L. Murchie, M.S. Robinson, B.W. Denevi, D.L. Domingue, Planet. Space Sci. 59, 1895 (2011). doi:10.1016/j.pss.2011.03.020

J.F. Kerridge, R.H. Becker, R.O. Pepin, Meteoritics 20, 682 (1985)

R.M. Killen, W.-H. Ip, Rev. Geophys. 37, 361 (1999)

R.M. Killen, T.H. Morgan, Icarus 101, 293 (1993)

R.M. Killen, M. Sarantos, NASA Lunar Science Institute Forum 2013 (2013). https://connect.arc.nasa.gov/ p9mo7206usy/

R.M. Killen, M. Sarantos, A.E. Potter, P. Reiff, Icarus 171, 1 (2004)

R.M. Killen, G. Cremonese, H. Lammer, S. Orsini, A.E. Potter, A.L. Sprague, P. Wurz, M.L. Khodachenko, H.I.M. Lichtenegger, A. Milillo, A. Mura, Space Sci. Rev. 132, 433 (2007)

B. Klecker, E. Möbius, M.A. Popecki, Space Sci. Rev. 124, 289 (2006)

R.L. Klima, N.R. Izenberg, S. Murchie, H.M. Meyer, K.R. Stockstill-Cahill, D.T. Blewett, M. D'Amore, B.W. Denevi, C.M. Ernst, J. Helbert, T.J. McCoy, A.L. Sprague, F. Vilas, S.Z. Weider, S.C. Solomon, Lunar Planet. Sci. 44, 1602 (2013)

A. Kotarba, I. Kruk, Z. Sojka, J. Catal. 221, 650 (2004)

U. Krähenbühl, A. Grütter, H.R. von Gunten, G. Meyer, F. Wegmüller, A. Wyttenback, Proc. Lunar Sci. Conf. 8, 3901 (1977)

G.Y. Kramer, S. Besse, D. Dhingra, J. Nettles, R. Klima, I. Garrick-Bethell, R.N. Clark, J.-P. Combe, J.W. Head III, L.A. Taylor, C.M. Pieters, J. Boardman, T.B. McCord, J. Geophys. Res. 116, E00G18 (2011). doi:10.1029/2010JE003729

H. Lammer, P. Wurz, M.R. Patel, R. Killen, C. Kolb, S. Massetti, S. Orsini, A. Milillo, Icarus 166, 238 (2003)

Y. Langevin, in Workshop on Lunar Breccias and Soils and Their Meteoritic Analogs. Tech. report 82-02, Lunar and Planetary Institute, Houston (1982), p. 87

J.C. Laul, J.J. Papike, S.B. Simon, Proc. Lunar Planet Sci. 12B, 389 (1981)

D.J. Lawrence, W.C. Feldman, J.O. Goldsten, T.J. McCoy, D.T. Blewett, W.V. Boynton, L.G. Evans, L.R. Nittler, E.A. Rhodes, S.C. Solomon, Icarus 209, 195 (2010)

D.J. Lawrence, W.C. Feldman, J.O. Goldsten, S. Maurice, P.N. Peplowski, B.J. Anderson, D. Bazell, R.L. McNutt Jr., L.R. Nittler, T.H. Prettyman, D.J. Rodgers, S.C. Solomon, S.Z. Weider, Science 339, 292 (2013)

F. Leblanc, R.E. Johnson, Icarus 164, 261 (2003)

S.M. Lederer, D.L. Domingue, F. Vilas, M. Abe, T.L. Farnham, K.S. Jarvis, S.C. Lowry, Y. Ohba, P.R. Weissman, L.M. French, H. Fukai, S. Hasegawa, M. Ishiguro, S.M. Larson, Y. Takagi, Icarus 173, 153 (2005)

C. Leinert, I. Richter, E. Pitz, B. Planck, Astron. Astrophys. 103, 177 (1981)

S.T. Lepri, T.H. Zurbuchen, L.A. Fisk, I.G. Richardson, H.V. Cane, G. Gloeckler, J. Geophys. Res. 106, $29231(2001)$ 
J.S. Lewis, Annu. Rev. Phys. Chem. 24, 339 (1973)

J.S. Lewis, in Mercury, ed. by F. Vilas, C.R. Chapman, M.S. Matthews (University of Arizona Press, Tucson, 1988), pp. 651-669

M. Lockwood, R. Stamper, M.N. Wild, A. Balogh, G. Jones, Astron. Geophys. 40, 10 (1999)

M.J. Loeffler, C.A. Dukes, W.Y. Chang, L.A. McFadden, R.A. Baragiola, Icarus 195, 622 (2008a). doi:10.1016/j.icarus.2008.02.002

M.J. Loeffler, R.A. Baragiola, M. Murayama, Icarus 196, 285 (2008b). doi:10.1016/j.icarus.2008.02.021

M.J. Loeffler, C.A. Dukes, R.A. Baragiola, J. Geophys. Res. 114, E03003 (2009). doi:10.1029/ 2008JE003249

S.G. Love, D.E. Brownlee, Science 262, 550 (1993)

P.G. Lucey, M.A. Riner, Icarus 212, 451 (2011)

A. Luhn, B. Klecker, D. Hovestadt, M. Scholer, G. Gloeckler, F.M. Ipavich, C.Y. Fan, L.A. Fisk, Adv. Space Res. 4, 161 (1984)

T.E. Madey, B.V. Yakshinskiy, V.N. Ageev, R.E. Johnson, J. Geophys. Res. 103, 5873 (1998)

T.E. Madey, R.E. Johnson, T.M. Orlando, Surf. Sci. 500, 838 (2002). doi:10.1016/S0039-6028(01)01556-4

A. Mallama, D. Wang, R.A. Howard, Icarus 155, 253 (2002)

R.H. Manka, in Photon and Particle Interactions with Surfaces in Space, ed. by R.J.L. Grard (Reidel, Dordrecht, 1973), pp. 347-361

I. Mann, H. Kimura, D.A. Biesecher, B.T. Tsurutani, E. Grun, R.B. McKibben, J.-C. Liou, R.M. MacQueen, T. Mukai, M. Guhathakurta, P. Lamy, Space Sci. Rev. 110, 269 (2004)

I. Mann, A. Czecjowski, N. Mayer-Vernet, A. Zaslavsky, H. Lamy, Plasma Physics and Controlled Fusion 52(12), 124012 (2010). doi:10.1088/0741-3335/52/12/124012

S. Marchi, A. Morbidelli, G. Cremonese, Astron. Astrophys. 431, 1123 (2005a)

S. Marchi, R. Bruneto, S. Magrin, M. Lazzarin, D. Gandolfi, Astron. Astrophys. 443, 769 (2005b)

S. Marchi, P. Paolicchi, M. Lazzarin, S. Magrin, Astron. J. 131, 297 (2006). doi:10.1086/498721

S. Marchi, S. Mottola, G. Cremonese, M. Massironi, E. Martellato, Astron. J. 137, 4936 (2009)

S. Marchi, W.F. Bottke, D.A. Kring, A. Morbidelli, Earth Planet. Sci. Lett. 325, 27 (2012)

S. Marchi, C.R. Chapman, C.I. Fassett, J.W. Head, W.F. Bottke, R.G. Strom, Nature 499, 59 (2013)

E. Marsch, K.-H. Mühlhäuser, R. Schwenn, H. Rosenbauer, K.-H. Muehlhaeuser, W. Pilipp, F.M. Neubauer, J. Geophys. Res. 87, 52 (1982)

S. Massetti, S. Orsini, A. Milillo, A. Mura, Planet. Space Sci. 55, 1557 (2007)

P. Maurer, P. Eberhardt, J. Geiss, N. Grogler, A. Stettler, G.M. Brown, A. Peckett, U. Krähenbühl, Geochim. Cosmochim. Acta 42, 1687 (1978)

B. Mauvernay, L. Presmanes, S. Capdeville, V.G. De Resende, E. De Grave, C. Bonningue, P. Tailhades, Thin Solid Films 515, 6532 (2007)

W.E. McClintock, M.R. Lankton, Space Sci. Rev. 131, 481 (2007)

W.E. McClintock, N.R. Izenberg, G.M. Holsclaw, D.T. Blewett, D.L. Domingue, J.W. Head, J. Helbert, T.J. McCoy, S.L. Murchie, M.S. Robinson, S.C. Solomon, A.L. Sprague, F. Vilas, Science 321, 62 (2008)

W.E. McClintock, R.J. Vervack Jr., E.T. Bradley, R.M. Killen, N. Mouawad, A.L. Sprague, M.H. Burger, S.C. Solomon, N.R. Izenberg, Science 324, 610 (2009)

D.J. McComas, H.A. Elliott, J.T. Gosling, D.B. Reisenfeld, R.M. Skoug, B.E. Goldstein, M. Neugebauer, A. Balogh, Geophys. Res. Lett. 29, 1290 (2002). doi:10.1029/2001GL014164

J.E. McCoy, D.R. Criswell, Proc. Lunar Sci. Conf. 5, 2991 (1974)

T.B. McCord, J.B. Adams, Science 178, 745 (1972a)

T.B. McCord, J.B. Adams, Icarus 17, 585 (1972b)

T.B. McCord, R.N. Clark, J. Geophys. Res. 84, 7664 (1979)

T.B. McCord, J.B. Adams, T.V. Johnson, Science 168, 1445 (1970)

T.B. McCord, T.M. Orlando, G. Teeter, G.B. Hansen, M.T. Sieger, N.G. Petrik, L. Van Keulen, J. Geophys. Res. 106, 3311 (2001). doi:10.1029/2000JE001282

T.B. McCord, L.A. Taylor, J.-P. Combe, G.Y. Kramer, C.M. Pieters, J.M. Sunshine, R.N. Clark, J. Geophys. Res. 116, E00G05 (2011). doi:10.1029/2010JE003711

T.B. McCord, J.-Y. Li, J.-P. Combe, H. McSween, R. Jaumann, V. Reddy, F. Tosi, D.A. Williams, D.T. Blewett, D. Turrini, E. Palomba, C.M. Pieters, M.C. DeSanctis, E. Ammanito, M.T. Capria, L. Le Corre, A. Longobardo, A. Nathues, D.W. Mittlefehldt, S. Schroder, H. Hiesinger, A.W. Beck, F. Capaccioni, U. Carsenty, H.U. Keller, B.W. Denevi, J.M. Sunshine, C.A. Raymond, C.T. Russell (Dawn Team), Nature 491, 83 (2012). doi:10.1038/nature11561

T.J. McCoy, T.L. Dickinson, G.E. Lofgren, Meteorit. Planet. Sci. 34, 735 (1999)

F.M. McCubbin, M.A. Riner, K.E. Vander Kaaden, L.K. Burkemper, Geophys. Res. Lett. 39, L09202 (2012). doi:10.1029/2012GL051711

A. McGuire, B. Hapke, Icarus 113, 134 (1995) 
D.S. McKay, G.H. Heiken, R.M. Taylor, U.S. Clanton, D.A. Morrison, G.H. Ladle, Proc. Lunar Sci. Conf. 3, 983 (1972)

D.S. McKay, G. Heiken, A. Basu, G. Blandfor, S. Simon, R. Reedy, B. French, J.J. Papike, in Lunar Sourcebook, ed. by G.H. Heiken, D.T. Vaniman, B.M. French (Cambridge University Press, New York, 1991), pp. 285-356

J.L. McLain, A.L. Sprague, G.A. Grieves, D. Schriver, P. Trávníček, T.M. Orlando, J. Geophys. Res. 116, E03007 (2011). doi:10.1029/2010JE003714

R.A. Mewaldt, Adv. Space Res. 14, 737 (1994)

R.A. Mewaldt, C.M.S. Cohen, G.M. Mason, A.C. Cummings, M.I. Desai, R.A. Leske, J. Raines, E.C. Stone, M.E. Wiedenbeck, T.T. von Rosenvinge, T.H. Zurbuchen, Space Sci. Rev. 130, 207 (2007)

R.A. Mewaldt, A.J. Davis, K.A. Lave, R.A. Leske, E.C. Stone, M.E. Wiedenbeck, W.R. Binns, E.R. Christian, A.C. Cummings, G.A. de Nolfo, M.H. Israel, A.W. Labrador, T.T. von Rosenvinge, Astrophys. J. Lett. 723, L1 (2010)

D. Mitchell, I. de Pater, Icarus 110, 2 (1994)

F. Miyake, K. Nagaya, K. Masuda, T. Nakamura, Nature 486, 240 (2012). doi:10.1039/nature11123

A. Morbidelli, H.F. Levison, K. Tsiganis, R. Gomes, Nature 435, 462 (2005). doi:10.1038/nature03540

A. Morbidelli, S. Marchi, W.F. Bottke, D.A. Kring, Earth Planet. Sci. Lett. 355, 144 (2012)

T.H. Morgan, H. Zook, A.E. Potter, Icarus 75, 156 (1988)

L.V. Moroz, A.V. Fisenko, L.F. Semjonova, C.M. Pieters, N.N. Korotaeva, Icarus 122, 366 (1996)

R.V. Morris, Proc. Lunar Sci. Conf. 7, 315 (1976)

R.V. Morris, Proc. Lunar Planet. Sci. Conf. 11, 1697 (1980)

R.V. Morris, R.V. Gibbons, F. Hörz, Geophys. Res. Lett. 2, 461 (1975)

R.V. Morris, R. Score, C. Dardano, G. Heiken, Handbook of Lunar Soils, Planetary Materials Branch Pub. 67 (NASA Johnson Space Center, Houston, 1983). 914 pp.

D. Morrison, Space Sci. Rev. 11, 271 (1970)

N. Mouawad, M.H. Burger, R.M. Killen, A.E. Potter, W.E. McClintock, R.J. Vervack Jr., E.T. Bradley, M. Benna, S. Naldu, Icarus 211, 21 (2011)

S.L. Murchie, T.R. Watters, M.S. Robinson, J.W. Head, R.G. Strom, C.R. Chapman, S.C. Solomon, W.E. McClintock, L.M. Prockter, D.L. Domingue, D.T. Blewett, Science 321, 73 (2008)

B.C. Murray, M.J. Belton, G.E. Danielson, M.E. Davies, D.E. Gault, B. Hapke, B. O’Leary, R.G. Strom, V. Suomi, N. Trask, Science 185, 169 (1974)

D. Nash, J. Geophys. Res. 72, 3089 (1967)

D. Nesvorny, D. Vokrouhlicky, W.F. Bottke, M. Sykes, Icarus 181, 107 (2006)

D. Nesvorny, D. Vokrouhlicky, A. Morbidelli, Astron. J. 133, 1962 (2007)

D. Nesvorny, P. Jenniskens, H.F. Levison, W.F. Bottke, D. Vokrouhlicky, M. Gounelle, Astrophys. J. 713, 816 (2010)

G. Neukum, Meteoritics 18, 362 (1983)

G. Neukum, B.A. Ivanov, in Hazards Due to Comets and Asteroids, ed. by T. Gehrels (University of Arizona Press, Tucson, 1994), pp. 359-416

G. Neukum, D.E. Wilhelms, Lunar Planet. Sci. 13, 590 (1982)

G.A. Neumann, J.F. Cavanaugh, X. Sun, E.M. Mazarico, D.E. Smith, M.T. Zuber, D. Mao, D.A. Paige, S.C. Solomon, C.M. Ernst, O.S. Barnouin, Science 339, 296 (2013)

L.R. Nittler, R.D. Starr, L. Lim, T.J. McCoy, T.H. Burbine, R.C. Reedy, J.I. Trombka, P. Borenstein, S.W. Squyres, W.V. Boynton, T.P. McClanahan, J.S. Bhangoo, P.E. Clark, M.E. Murphy, R. Killen, Meteorit. Planet. Sci. 36, 1673 (2001). doi:10.1111/j.1945-5100.tb01856.x

L.R. Nittler, R.D. Starr, S.Z. Weider, T.J. McCoy, W.V. Boynton, D.S. Ebel, C.M. Ernst, L.G. Evans, J.O. Goldsten, D.K. Hamara, D.J. Lawrence, R.L. McNutt Jr., C.E. Schlemm II, S.C. Solomon, A.L. Sprague, Science 333, 1847 (2011)

S.K. Noble, C.M. Pieters, Astron. Vestn. 37, 34 (2003). English transl. in Solar Sys. Res. 37, 31

S.K. Noble, C.M. Pieters, L.A. Taylor, R.V. Morris, A.C. Carlton, D.S. McKay, L.P. Keller, Meteorit. Planet. Sci. 36, 31 (2001)

S.K. Noble, C.M. Pieters, L.P. Keller, Icarus 192, 629 (2007)

S.K. Noble, L.P. Keller, C.M. Pieters, Meteorit. Planet. Sci. 45, 2007 (2011)

T. Noguchi, T. Nakamura, M. Kimura, M.E. Zolensky, M. Tanaka, T. Hashimoto, M. Kono, A. Nakato, T. Ogami, A. Fujimura, M. Abe, T. Yada, T. Mukai, M. Ueno, T. Okada, K. Shirai, Y. Ishibashi, R. Okazaki, Science 333, 1121 (2011)

T. Noguchi, M. Kimura, T. Hashimoto, M. Konno, T. Nakamura, A. Nakato, T. Ogami, H. Ishida, R. Sagae, S. Tsujimoto, A. Tsachiyama, M.E. Zolensky, M. Tanaka, A. Fujimura, M. Abe, T. Yada, T. Mukai, M. Ueno, T. Okada, K. Shirai, Y. Ishibushi, R. Okazaki, Lunar Planet. Sci. 43, 1896 (2012) 
T. Noguchi, M. Kimura, T. Hashimoto, M. Konno, T. Nakamura, M.E. Zolensky, R. Okazaki, M. Tanaka, A. Tsuchiyama, A. Nakato, T. Ogami, H. Ishida, R. Sagae, S. Tsujimoto, T. Matsumoto, J. Matsuno, A. Fujimura, M. Abe, T. Yada, T. Mukai, M. Ueno, T. Okada, K. Shirai, Y. Ishibashi, Meteorit. Planet. Sci. 49, 188 (2014). doi:10.1111/maps.12111

D.A. Paige, M.A. Siegler, J.K. Harmon, G.A. Neumann, E.M. Mazarico, D.E. Smith, M.T. Zuber, E. Harju, M.L. Delitsky, S.C. Solomon, Science 339, 300 (2013)

D.A. Paige, S.E. Wood, A.R. Vasavada, Science 258, 643 (1992)

D.A. Papanastassiou, G.J. Wasserburg, Earth Planet. Sci. Lett. 12, 36 (1971a)

D.A. Papanastassiou, G.J. Wasserburg, Earth Planet. Sci. Lett. 11, 37 (1971b)

J.J. Papike, S.B. Simon, C. White, J.C. Laul, Proc. Lunar Planet Sci. 12B, 409 (1981)

J.J. Papike, S.B. Simon, J.C. Laul, Rev. Geophys. Space Phys. 20, 761 (1982)

J. Papike, L. Taylor, S. Simon, in Lunar Sourcebook, ed. by G.H. Heiken, D.T. Vaniman, B.M. French (Cambridge University Press, New York, 1991), pp. 121-182

P.N. Peplowski, L.G. Evans, S.A. Hauck II, T.J. McCoy, W.V. Boynton, J.J. Gillis-Davis, D.S. Ebel, J.O. Goldsten, D.K. Hamara, D.J. Lawrence, R.L. McNutt Jr., L.R. Nittler, S.C. Solomon, E.A. Rhodes, A.L. Sprague, R.D. Starr, K.R. Stockstill-Cahill, Science 333, 1850 (2011)

P.N. Peplowski, D.J. Lawrence, E.A. Rhodes, A.L. Sprague, T.J. McCoy, B.W. Denevi, L.G. Evans, J.W. Head, L.R. Nittler, S.C. Solomon, K.R. Stockstill-Cahill, S.Z. Weider, J. Geophys. Res. 117, E00L04 (2012). doi:10.1029/2012JE004141

P.N. Peplowski, L.G. Evans, K.R. Stockstill-Cahill, D.J. Lawrence, T.J. McCoy, L.R. Nittler, S.C. Solomon, A.L. Sprague, R.D. Starr, S.Z. Weider, Icarus 228, 86 (2014)

C.M. Pieters, J. Geophys. Res. 88, 9534 (1983)

C.M. Pieters, in Remote Geochemical Analysis: Elemental and Mineralogical Composition, ed. by C.M. Pieters, P.A.J. Englert (Cambridge University Press, New York, 1993), pp. 309-339

C.M. Pieters, L.A. Taylor, Geophys. Res. Lett. 30, 2048 (2003). doi:10.1029/2003GL018212

C.M. Pieters, E.M. Fischer, O. Rode, A. Basu, J. Geophys. Res. 98, 20817 (1993)

C.M. Pieters, L.A. Taylor, S.K. Noble, L.P. Keller, B. Hapke, R.V. Morris, C.C. Allen, D.S. McKay, S. Wentworth, Meteorit. Planet. Sci. 35, 1101 (2000)

C.M. Pieters, J.N. Goswami, R.N. Clark, M. Annadurai, J. Boardman, B. Buratti, J.-P. Combe, M.D. Dyar, R. Green, J.W. Head, C. Hibbits, M. Hicks, P. Isaacson, R. Klima, G. Kramer, S. Kumar, E. Livo, S. Lundeen, E. Malaret, T. McCord, J. Mustard, J. Nettles, N. Petro, C. Runyon, M. Staid, J. Sunshine, L.A. Taylor, S. Tompkins, P. Varanasi, Science 326, 568 (2009)

C.M. Pieters, E. Ammannito, D.T. Blewett, B.W. Denevi, M.C. DeSanctis, M.J. Gaffey, L. Le Corre, J.-Y. Li, S. Marchi, T.B. McCord, L.A. McFadden, D.W. Mittlefehldt, A. Nathues, E. Palmer, V. Reddy, C.A. Raymond, C.T. Russell, Nature 491, 79 (2012). doi:10.1038/nature11534

A.E. Potter, Geophys. Res. Lett. 22, 3289 (1995)

A. Potter, T. Morgan, Science 229, 651 (1985)

A.E. Potter, T.H. Morgan, Icarus 67, 336 (1986)

T.H. Prettyman, D.W. Mittlefehldt, N. Yamashita, D.J. Lawrence, A.W. Beck, W.C. Feldman, T.J. McCoy, H.Y. McSween, M.J. Toplis, T.N. Titus, P. Tricarico, R.C. Reedy, J.S. Hendricks, O. Forni, L. Le Corre, J.-Y. Li, H. Mizzon, V. Reddy, C.A. Raymond, C.T. Russell, Science 338, 242 (2012)

T.I. Pulkkinen, H. Nevanlinna, P.J. Pulkkinen, M. Lockwood, Space Sci. Rev. 95, 625 (2001)

M.E. Purucker, T.J. Sabaka, S.C. Solomon, B.J. Anderson, H. Korth, M.T. Zuber, G.A. Neumann, Earth Planet. Sci. Lett. 285, 340 (2009)

G.M. Raisebeck, F. Yiou, D. Bourles, C. Lorius, J. Jouzel, N.I. Barkov, Nature 326, 273 (1987)

D.V. Reames, C.K. Ng, A.J. Tylka, Geophys. Res. Lett. 26, 3585 (1999)

G.W. Reed Jr., Meteorit. Planet. Sci. 34, 809 (1999)

J.J. Rennilson, D.R. Criswell, Moon 10, 121 (1974)

E.A. Rhodes, L.G. Evans, L.R. Nittler, R.D. Starr, A.L. Sprague, D.J. Lawrence, T.J. McCoy, K.R. StockstillCahill, J.O. Goldsten, P.N. Peplowski, W.V. Boynton, S.C. Solomon, Planet. Space Sci. 59, 1829 (2011)

I. Ribas, E.F. Guinan, M. Güdel, M. Audard, Astrophys. J. 622, 680 (2005)

N.C. Richmond, L.L. Hood, D.L. Mitchell, R.P. Lin, M.H. Acuña, A.B. Binder, J. Geophys. Res. 110, E05011 (2005). doi:10.1029/2005JE002405

M.A. Riner, P.G. Lucey, S.J. Desch, F.M. McCubbin, Geophys. Res. Lett. 36, L02201 (2009). doi:10.1029/ 2008GL036128

M.S. Robinson, S.L. Murchie, D.T. Blewett, D.L. Domingue, S.E. Hawkins III, J.W. Head, G.M. Holsclaw, W.E. McClintock, T.J. McCoy, R.L. McNutt Jr., L.M. Prockter, S.C. Solomon, T.R. Watters, Science 321, 66 (2008)

D.L. Rosenberg, G.K. Wehner, J. Geophys. Res. 69, 3307 (1964)

A.P. Rouillard, M. Lockwood, I. Finch, J. Geophys. Res. 112, A05103 (2007). doi:10.1029/2006JA012130 
C.T. Russell, P.J. Coleman Jr., B.K. Fleming, L. Hilburn, G. Ioannidis, B.R. Lichtenstein, G. Schubert, Proc. Lunar Sci. Conf. 6, 2955 (1975)

G. Ryder, Eos 71, 313 (1990). doi:10.1029/90EO00086

G. Ryder, J. Geophys. Res. 107, 5022 (2002). doi:10.1029/2001JE001583

M. Sarantos, R.M. Killen, D. Kim, Planet. Space Sci. 55, 1584 (2007)

M. Sarantos, R.M. Killen, A.S. Sharma, J.A. Slavin, Geophys. Res. Lett. 35, L04105 (2008). doi:10.1029/ 2007GL032310

M. Sarantos, R.M. Killen, W.E. McClintock, E.T. Bradley, R.J. Vervack Jr., M. Benna, J.A. Slavin, Planet. Space Sci. 59, 1992 (2011)

S. Sasaki, E. Kurahashi, Adv. Space Res. 33, 2152 (2004)

S. Sasaki, K. Nakamura, Y. Hamabe, E. Kurahashi, T. Hiroi, Nature 410, 555 (2001)

S. Sasaki, T. Hiroi, K. Nakamura, Y. Hamabe, E. Kurahashi, M. Yamada, Adv. Space Res. 29, 783 (2002)

S. Sasaki, E. Kurahashi, C. Yamanaka, K. Nakamura, Adv. Space Res. 31, 2537 (2003)

D.J. Scheeres, C.M. Hartzell, P. Sanchez, M. Swift, Icarus 210, 968 (2010)

D. Schriver, P. Trávníček, M. Ashour-Abdalla, R.L. Richard, P. Hellinger, J.A. Slavin, B.J. Anderson, D.N. Baker, M. Benna, S.A. Boardsen, R.E. Gold, G.C. Ho, H. Korth, S.M. Krimigis, W.E. McClintock, T.M. Orlando, M. Sarantos, A.L. Sprague, R.D. Starr, Planet. Space Sci. 59, 2026 (2011a). doi:10.1016/j.pss.2011.03.008

D. Schriver, P. Trávníček, B.J. Anderson, M. Ashour-Abdalla, D.N. Baker, M. Benna, S.A. Boardsen, R.E. Gold, P. Hellinger, G.C. Ho, H. Korth, S.M. Krimigis, R.L. McNutt Jr., J.M. Raines, R.L. Richard, J.A. Slavin, S.C. Solomon, R.D. Starr, T.H. Zurbuchen, Geophys. Res. Lett. 38, L23103 (2011b). doi:10.1029/2011GL049629

N.A. Schwadron, G. Gloeckler, Space Sci. Rev. 130, 293 (2007)

R. Schwenn, in Physics of the Inner Heliosphere I, Space and Solar Physics, vol. 20, ed. by R. Schwenn, E. Marsch (Springer, Berlin, 1990), pp. 99-181

Y. Shao, J. Paul, Appl. Surf. Sci. 72, 113 (1993)

M.K. Shepard, P. Helfenstein, J. Geophys. Res. 112, E03001 (2007). doi:10.1029/2005JE002625

R.W. Siegfried II, S.C. Solomon, Icarus 23, 192 (1974)

L. Siess, E. Dufour, M. Forestini, Astron. Astrophys. 358, 593 (2000)

J.A. Simpson, J.H. Eraker, J.E. Lamport, P.H. Walpole, Science 185, 160 (1974)

R.B. Singer, T.L. Roush, J. Geophys. Res. 90, 12434 (1985)

B. Siskind, D. Gruen, R. Varma, J. Vac. Sci. Technol. 14, 537 (1977)

M.A. Slade, B.J. Butler, D.O. Muhleman, Science 258, 635 (1992)

J.A. Slavin, R.E. Holzer, J. Geophys. Res. 84, 2076 (1979)

J.A. Slavin, M.H. Acuña, B.J. Anderson, D.N. Baker, M. Benna, G. Gloeckler, R.E. Gold, G.C. Ho, R.M. Killen, H. Korth, S.M. Krimigis, R.L. McNutt Jr., L.R. Nittler, J.M. Raines, D. Schriver, S.C. Solomon, R.D. Starr, P. Trávníček, T.H. Zurbuchen, Science 321, 85 (2008)

J.A. Slavin, M.H. Acuña, B.J. Anderson, D.N. Baker, M. Benna, S.A. Boardsen, G. Gloeckler, R.E. Gold, G.C. Ho, H. Korth, S.M. Krimigis, R.L. McNutt Jr., J.M. Raines, M. Sarantos, D. Schriver, S.C. Solomon, P. Trávníček, T.H. Zurbuchen, Science 324, 606 (2009)

J.A. Slavin, R.P. Lepping, C.-C. Wu, B.J. Anderson, D.N. Baker, M. Benna, S.A. Boardsen, R.M. Killen, H. Korth, S.M. Krimigis, W.E. McClintock, R.L. McNutt Jr., M. Sarantos, D. Schriver, S.C. Solomon, P. Trávníček, T.H. Zurbuchen, Geophys. Res. Lett. 37, L02105 (2010a). doi:10.1029/2009GL041485

J.A. Slavin, B.J. Anderson, D.N. Baker, M. Benna, S.A. Boardsen, G. Gloeckler, R.E. Gold, G.C. Ho, H. Korth, S.M. Krimigis, R.L. McNutt Jr., L.R. Nittler, J.M. Raines, M. Sarantos, D. Schriver, S.C. Solomon, R.D. Starr, P. Travnicek, T.H. Zurbuchen, Science 329, 665 (2010b)

J.A. Slavin, G.A. DiBraccio, J.M. Raines, T. Zurbuchen, G. Gloeckler, S.A. Boardsen, T. Sundberg, B.J. Anderson, H. Korth, G.C. Ho, S.M. Krimigis, R.L. McNutt, D.N. Baker, S.C. Solomon, Geophys. Res. Abstr. 14, EGU2012-3817-EGU2012-3 (2012)

S.C. Solomon, Icarus 28, 509 (1976)

S.C. Solomon, R.L. McNutt Jr., T.R. Watters, D.J. Lawrence, W.C. Feldman, J.W. Head, S.M. Krimigis, S.L. Murchie, R.J. Phillips, J.A. Slavin, M.T. Zuber, Science 321, 59 (2008)

C.P. Sonett, G.E. Morfill, R.J. Jokipii, Nature 330, 458 (1987)

A.L. Sprague, R.W.H. Kozlowski, D.M. Hunten, Bull. Am. Astron. Soc. 21, 974 (1989)

A.L. Sprague, R.W.H. Kozlowski, F.C. Witteborn, D.P. Cruikshank, D.H. Wooden, Icarus 109, 156 (1994)

A.L. Sprague, D.M. Hunten, K. Lodders, Icarus 118, 211 (1995)

A.L. Sprague, J.P. Emery, K.L. Donaldson, R.W. Russell, D.K. Lynch, A.L. Mazuk, Meteorit. Planet. Sci. 37, 1255 (2002)

A.L. Sprague, J. Warell, G. Cremonese, Y. Langevin, J. Helbert, P. Wurz, I. Veselovsky, S. Orsini, A. Milillo, Space Sci. Rev. 132, 399 (2007) 
A.L. Sprague, K.L. Donaldson Hanna, R.W.H. Kozlowski, J. Helbert, A. Maturilli, J.B. Warell, J.L. Hora, Planet. Space Sci. 57, 364 (2009)

R.D. Starr, D. Schriver, L.R. Nittler, S.Z. Weider, P.K. Byrne, G.C. Ho, E.A. Rhodes, C.E. Schlemm II, S.C. Solomon, P.M. Trávníček, J. Geophys. Res. 117, E002L02 (2012). doi:10.1029/2012JE004118

L.V. Starukhina, Adv. Space Res. 37, 50 (2006)

L. Starukhina, Y.G. Shkuratov, Lunar Planet. Sci. 34, 1258 (2003)

F. Steinhilber, J.A. Abreu, J. Beer, Astrophys. Space Sci. Trans. 4, 1 (2008). doi:10.5194/astra-4-1-2008

K.R. Stockstill-Cahill, T.J. McCoy, L.R. Nittler, S.Z. Weider, S.A. Hauck II, J. Geophys. Res. 117, E00L15 (2012). doi:10.1029/2012JE004140

D. Stöffler, G. Ryder, Space Sci. Rev. 96, 9 (2001)

D. Stöffler, A. Dischoff, V. Buchwald, A.E. Rugin, in Meteorites and the Early Solar System, ed. by J.F. Kerridge, M.S. Matthews (University of Arizona Press, Tucson, 1988), pp. 165-202

D. Stöffler, K. Keil, E.R.D. Scott, Geochim. Cosmochim. Acta 55, 3845 (1991)

G. Strazzulla, E. Dotto, R. Binzel, R. Burnetto, M.A. Barucci, A. Blanco, V. Orofino, Icarus 174, 31 (2005)

R.G. Strom, A.L. Sprague, Exploring Mercury: The Iron Planet (Springer, New York, 2003). 216 pp.

R.G. Strom, R. Malhotra, T. Ito, F. Yoshida, D.A. Kring, Science 309, 1847 (2005). doi:10.1126/ science. 1113544

T.J. Stubbs, R.R. Vondrak, W.M. Farell, Adv. Space Sci. 37, 59 (2006)

J.M. Sunshine, T.L. Farnham, L.M. Feaga, O. Groussin, F. Merlin, R.E. Miliken, M.F. A'Hearn, Science 326, 565 (2009)

S.P. Swordy, Space Sci. Rev. 99, 85 (2001)

L.A. Taylor, R. Rossman, Q. Qi, Lunar Planet. Sci. 26, 1399 (1995)

L.A. Taylor, R.V. Morris, L.P. Keller, C.M. Pieters, A. Patchen, D.-H. Taylor, S.J. Wentworth, D.S. McKay, Lunar Planet. Sci. 31, 1842 (2000)

L.A. Taylor, C.M. Pieters, L.P. Keller, R.V. Morris, D.S. McKay, A. Patchen, S.J. Wentworth, Meteorit. Planet. Sci. 36, 288 (2001a)

L.A. Taylor, C.M. Pieters, L.P. Keller, R.V. Morris, D.S. McKay, Geophys. Res. Lett. 106, 27985 (2001b)

L.A. Taylor, C.M. Pieters, A. Patchen, D.-H.S. Taylor, R.V. Morris, L.P. Keller, D.S. McKay, J. Geophys. Res. 115, E02002 (2010). doi:10.1029/2009JE003427

P. Trávníček, P. Hellinger, D. Schriver, Geophys. Res. Lett. 34, L05104 (2007). doi:10.1029/2006GL028518

P. Trávníček, P. Hellinger, D. Schriver, D. Hercik, J.A. Slavin, B.J. Anderson, Geophys. Res. Lett. 36, L07104 (2009). doi:10.1029/2008GL036630

P. Trávníček, D. Schriver, P. Hellinger, D. Hercik, B.J. Anderson, M. Sarantos, J.A. Slavin, Icarus 209, 11 (2010)

J.I. Trombka, S.W. Squyres, J. Bruckner, W.V. Boynton, R.C. Reedy, T.J. McCoy, P. Gorenstein, L.G. Evans, J.R. Arnold, R.D. Starr, L.R. Nittler, M.E. Murphy, I. Mikheeva, R.L. McNutt, T.P. McClanahan, E. McCarthy, J.O. Goldstein, R.E. Gold, S.R. Floyd, P.E. Clark, T.H. Burbine, J.S. Bhangoo, S.H. Bailey, M. Petaev, Science 289, 2101 (2000). doi:10.1126/science.289.5487.2101

G. Turner, P.H. Cadogan, C.J. Yonge, Nature 242, 513 (1973)

A.R. Vasavada, D.A. Paige, S.E. Wood, Icarus 141, 179 (1999)

P. Vernazza, R.P. Binzel, A. Rossi, M. Fulchignoni, M. Birlan, Nature 458, 993 (2009)

F. Vilas, Icarus 64, 133 (1985)

F. Vilas, T.B. McCord, Icarus 28, 593 (1976)

F. Vilas, E.A. Jensen, D.L. Domingue, L.A. McFadden, C. Runyon, W.W. Mendell, Earth Planets Space 60, 67 (2008)

A.P. Vinogradov, V.I. Nefedov, V.S. Urusov, N.M. Zhavoronkov, Proc. Lunar Sci. Conf. 3, 1421 (1972)

R. von Steiger, J. Geiss, Adv. Space Res. 13, 63 (1993)

R. von Steiger, N.A. Schwadron, L.A. Fisk, J. Geiss, G. Gloeckler, S. Hefti, B. Wilken, R.F. WimmerSchweingruber, T.H. Zurbuchen, J. Geophys. Res. 105, 27217 (2000)

R. von Steiger, T.H. Zurbuchen, D.J. McComas, Geophys. Res. Lett. 37, L22101 (2010). doi:10.1029/ 2010GL045389

Y.-M. Wang, N.R. Sheeley, Astrophys. J. 355, 726 (1990)

J. Warell, Icarus 161, 199 (2003)

J. Warell, Icarus 167, 271 (2004)

J. Warell, D.T. Blewett, Icarus 168, 257 (2004)

J. Warell, A.L. Sprague, J.P. Emery, R.W.H. Kozlowski, A. Long, Icarus 180, 281 (2006)

J. Warell, A. Sprague, R. Kozlowski, D.A. Rothery, N. Lewis, J. Helbert, E. Cloutis, Icarus 209, 138 (2010)

G.J. Wasserburg, D.A. Papanastassiou, Earth Planet. Sci. Lett. 13, 97 (1971)

T.R. Watters, S.C. Solomon, M.S. Robinson, J.W. Head, S.L. Andre, S.A. Hauck, S.L. Murchie, Earth Planet. Sci. Lett. 285, 283 (2009) 
G.K. Wehner, in The Lunar Surface Layer, ed. by J.W. Salisbury, P.E. Glaser (Academic Press, San Diego, 1964), pp. 313-322

S.J. Weidenschilling, Icarus 35, 99 (1978)

S.Z. Weider, L.R. Nittler, R.D. Starr, T.J. McCoy, K.R. Stockstill-Cahill, P.K. Byrne, B.W. Denevi, J.W. Head, S.C. Solomon, J. Geophys. Res. 117, E00L05 (2012). doi:10.1029/2012JE004153

S.Z. Weider, L.R. Nittler, R.D. Starr, S.C. Solomon, Lunar Planet. Sci. 44, 2189 (2013)

S.J. Wentworth, L.P. Keller, D.S. McKay, R.V. Morris, Meteorit. Planet. Sci. 34, 593 (1999)

G.W. Wetherill, in Mercury, ed. by F. Vilas, C.R. Chapman, M.S. Matthews (University of Arizona Press, Tucson, 1988), pp. 670-691

R.C. Wiens, P. Bochsler, D.S. Burnett, R.F. Wimmer-Schweingruber, Earth Planet. Sci. Lett. 226, 549 (2004). doi:10.1016/j.epls.2004.07.011

M. Willman, R. Jedicke, D. Nesvorny, N. Moskovita, I. Zeljko, R. Fevig, Icarus 195, 663 (2008)

R.M. Winslow, C.L. Johnson, B.J. Anderson, H. Korth, J.A. Slavin, M.E. Purucker, S.C. Solomon, Geophys. Res. Lett. 39, L08112 (2012). doi:10.1029/2012GL051472

R.M. Winslow, B.J. Anderson, C.L. Johnson, J.A. Slavin, H. Korth, M.E. Purucker, D.N. Baker, S.C. Solomon, J. Geophys. Res. Space Phys. 118, 2213 (2013)

B.E. Wood, Space Sci. Rev. 126, 3 (2006)

B.E. Wood, J.L. Linsky, H.-R. Müller, G.P. Zank, Astrophys. J. 547, L49 (2001)

B.E. Wood, H.-R. Müller, G. Zank, J.L. Linsky, Astrophys. J. 574, 412 (2002)

P. Wurz, H. Lammer, Icarus 164, 1 (2003)

P. Wurz, U. Rohner, J.A. Whitby, C. Kolb, H. Lammer, P. Dobnikar, J.A. Martín-Fernández, Icarus 191, 486 (2007)

P. Wurz, J.A. Whitby, U. Rohner, J.A. Martín-Fernández, H. Lammer, C. Kolb, Planet. Space Sci. 58, 1599 (2010)

B.V. Yakshinskiy, T.E. Madey, Surf. Sci. 451, 160 (2000)

B.V. Yakshinskiy, T.E. Madey, Surf. Sci. 528, 54 (2003)

B.V. Yakshinskiy, T.E. Madey, Icarus 168, 53 (2004)

B.V. Yakshinskiy, T.E. Madey, Surf. Sci. 593, 202 (2005)

M. Yamada, S. Sasaki, H. Nagahara, A. Fujiwara, S. Hasegawa, H. Yano, T. Hiroi, H. Ohashi, H. Otake, Earth Planets Space 51, 1255 (1999)

J.H. Yao, K.R. Eldar, H. Guo, M. Grant, Phys. Rev. B 47, 14110 (1993)

L. Yin, T. Tsang, Proc. Lunar Sci. Conf. 7, 891 (1976)

L. Yin, S. Ghose, I. Adler, J. Geophys. Res. 77, 1360 (1972)

L. Yin, T. Tsang, I. Adler, Proc. Lunar Sci. Conf. 6, 3277 (1975)

G.P. Zank, P.C. Frisch, Astrophys. J. 518, 965 (1999)

E. Zellner, L. Ronca, P. Levy, J. Geophys. Res. 71, 4855 (1996)

L. Zhao, T.H. Zurbuchen, L.A. Fisk, Geophys. Res. Lett. 36, L14104 (2009). doi:10.1029/2009GL039181

H.A. Zook, Proc. Lunar Sci. Conf. 6, 1653 (1975)

H.A. Zook, J.E. McCoy, Geophys. Res. Lett. 18, 2117 (1991)

T.H. Zurbuchen, L.A. Fisk, G. Gloeckler, R. von Steiger, Geophys. Res. Lett. 29, 1352 (2002). doi:10.1029/ 2001GL013946

T.H. Zurbuchen, J.M. Raines, D.J. Gershman, J.A. Gilbert, G. Gloeckler, B.J. Anderson, D.N. Baker, H. Korth, S.M. Krimigis, M. Sarantos, D. Schriver, R.L. McNutt Jr., S.C. Solomon, Science 333, 1862 (2011) 